\title{
Kinematic Calibration of Serial Manipulators using Relative Measurements
}

by

David Chao-Chia Lu, B.Eng.

\author{
A Thesis submitted to \\ the Faculty of Graduate and Postdoctoral Affairs \\ in partial fulfilment of \\ the requirements for the degree of \\ Master of Applied Science
}

\author{
Ottawa-Carleton Institute for \\ Mechanical and Aerospace Engineering \\ Department of Mechanical and Aerospace Engineering \\ Carleton University \\ Ottawa, Ontario, Canada \\ January, 2014 \\ Copyright (C) \\ 2014 - David Chao-Chia Lu
}


The undersigned hereby recommends to the

Faculty of Graduate and Postdoctoral Affairs

acceptance of the thesis

\title{
Kinematic Calibration of Serial Manipulators using Relative Measurements
}

\author{
submitted by David Chao-Chia Lu, B.Eng. \\ in partial fulfillment of the requirements for the degree of \\ Master of Applied Science
}

Prof. M.J.D. Hayes, Thesis Supervisor

Prof. M. Yaras, Chair,

Department of Mechanical and Aerospace Engineering

Ottawa-Carleton Institute for Mechanical and Aerospace Engineering

Department of Mechanical and Aerospace Engineering

Carleton University

January, 2014 


\section{Abstract}

Kinematic calibration is necessary to enhance the accuracy of robotic manipulators. It is typically desired to perform this task in both a cost-effective and time-efficient manner. Many techniques exist in the literature that achieve both goals.

In this thesis, a novel model-based kinematic calibration method using relative measurements is developed and implemented, which has proven to be useful for optical measurements, and is capable of achieving the same level of accuracy as the typical absolute measurement methods. Furthermore, the effects of measurement noise, the number of measured poses, and the best pose configurations for kinematic calibration are investigated.

Both a Thermo CRS A465 and a 7 DOF WAM Arm were used in this thesis. The results for the WAM Arm lead to a significant improvement in the end-effector pose accuracy. The implication is that the relative measurement concept is a valid tool for model-based kinematic calibration of serial manipulators. 


\section{Acknowledgments}

I would like to start off by thanking my supervisor Prof. John Hayes for his advice, guidance, encouragement, and support throughout this entire thesis work. He also provided the perfect topic for me to work with, which contains both theoretical and practical components.

I would also like to thank the MAAE office for guidance on completing the requirement of this degree over the years. Also, my friends and colleagues at Carleton University, they shared their insights and experience on the courses and the work that they have done, but more importantly they made my university career a lot more interesting.

Finally, I would like to thank my parents, whom decided to send both their kids (my brother and I) to Canada for education since high school. They are willing to live apart from their children for enforcing the entire family's belief of "better

education". I could honestly say that I would never be where I am right now if they hadn't supported this decision. 


\section{Table of Contents}

Abstract iii

Acknowledgments iv

Table of Contents $\quad$ v

List of Tables $\quad$ ix

List of Figures $\quad$ xi

1 Introduction 1

1.1 Motivation . . . . . . . . . . . . . . . . . . . . . 1

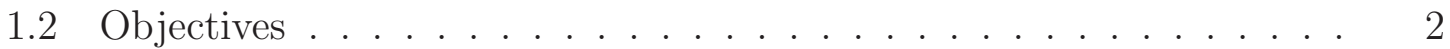

1.3 Scope and Contributions to Knowledge . . . . . . . . . . . . 3

1.4 Overview . . . . . . . . . . . . . . . . . . . . 4

2 Background and Literature Review $\quad 6$

2.1 Under the Hood of Kinematic Calibration . . . . . . . . . . 6

2.2 Literature Review of Kinematic Calibration of 6R Serial Manipulators 10

2.2.1 Kinematic Calibration Method . . . . . . . . . . . . 10

2.2 .2 Measurement Method . . . . . . . . . . . . . . . . . 14

2.2 .3 Kinematic Modelling . . . . . . . . . . . . . . 18 
2.3 Identifying Kinematic Parameters . . . . . . . . . . . . . . . . 22

2.3.1 Nonlinear Least-Squares: Gradient Based Search . . . . . . . . 22

2.3.2 Genetic Algorithm . . . . . . . . . . . . . . . . . . . . 29

2.3.3 Pseudoinverse using Singular Value Decomposition . . . . . . 31

2.3.4 Numerical Conditioning for Least-Squares Solutions . . . . . . 34

2.3.5 Numerical Conditioning using SVD . . . . . . . . . . . . . 34

3 Kinematic Modelling $\quad 36$

3.1 Properties of a Good Kinematic Model . . . . . . . . . . . . . . 36

3.2 Forward Kinematics . . . . . . . . . . . . . . . . . . . . . 37

3.2.1 Base Coordinate Transformation _. . . . . . . . . 37

3.2.2 End Link Coordinate Transformation . . . . . . . . . . . 38

3.2.3 Forward Kinematics for Serial Manipulators . . . . . . . . . 39

3.3 Jacobian Matrix for Kinematic Calibration . . . . . . . . . . . 40

3.3.1 Analytical Jacobian Matrix . . . . . . . . . . . . . . 40

3.3 .2 Geometric Jacobian Matrix _. . . . . . . . . . . . . . 42

3.3.3 Deriving the Geometric Jacobian from the Analytical Jacobian 44

3.3.4 Jacobian for the Hayati Parameters . . . . . . . . . . . . 46

3.4 Differential Kinematics for the Objective Function . . . . . . . . . 47

4 Simulations For Kinematic Calibration $\quad 50$

4.1 The Kinematic Models . . . . . . . . . . . . . . . . . . . . 50

4.2 Simulation Example . . . . . . . . . . . . . . . . . . . 52

4.2 .1 Measurements . . . . . . . . . . . . . . . . . . . . 53

4.2.2 Absolute Measurement Results . . . . . . . . . . . . . . 53

4.2.3 Relative Measurement Results . . . . . . . . . . . . . . . . 56

4.3 Observation Strategy . . . . . . . . . . . . . . . . . . . 58 
4.3.1 Conditioning the Jacobian Matrix . . . . . . . . . . . . . . 59

4.3.2 Measurement Noise . . . . . . . . . . . . . . . . . . 59

4.3.3 Number of Measurement Poses . . . . . . . . . . . . . . 66

4.3.4 Pose Selection . . . . . . . . . . . . . . . . . . 67

5 Experimental Setup and Results $\quad 78$

5.1 End-Effector Pose Measurements . . . . . . . . . . . . . . . . 78

5.1.1 Camera Calibration . . . . . . . . . . . . . 79

5.1.2 Camera Calibration Results . . . . . . . . . . . . . . 82

5.2 Experiment using the Thermo CRS A465 . . . . . . . . . . . . 84

5.2.1 Thermo CRS A465 Programming . . . . . . . . . . . 84

5.2.2 End-Effector Pose Measurements . . . . . . . . . . . . . . . 85

5.2.3 A465 Kinematic Calibration Results . . . . . . . . . . . . . . 87

5.3 Experiment using WAM Arm . . . . . . . . . . . . . 88

5.3.1 Generating Pose Trajectory . . . . . . . . . . . . 88

5.3.2 Kinematic Calibration Results of the WAM . . . . . . . . 92

6 Conclusion and Future Work $\quad 98$

6.1 Concluding Remarks . . . . . . . . . . . . . . . . . . . . . 98

6.2 Suggestions for Future Work . . . . . . . . . . . . . . . . . . 101

Appendix A Spatial Displacements Representations 108

A.1 Rotation of a Rigid Body . . . . . . . . . . . . . . . 109

A.1.1 Rotation Matrix . . . . . . . . . . . . . . . . . . 110

A.1.2 Euler Angles. . . . . . . . . . . . . . . . . . . . . 110

A.1.3 Angle-Axis . . . . . . . . . . . . . . . . . . . 113

A.1.4 Unit Quaternion ................ 114 
A.2 Homogeneous Coordinates . . . . . . . . . . . . . . . . 116

A.3 Homogeneous Transformation . . . . . . . . . . . . . . . . 117

A.3.1 3D Pose Transformation . . . . . . . . . . . . . . . 118

A.3.2 6D Pose Transformations . . . . . . . . . . . . . . . . . . 119

A.4 Relative Displacements using Homogeneous Transformation Matrix . 120

Appendix B Links Velocities Representations 122

B.1 Derivative of a Rotation Matrix . . . . . . . . . . . . . . 122

B.2 Link Velocities . . . . . . . . . . . . . . . . . . . 123

Appendix C MATLAB Code for the Experiments $\quad 126$

C.1 The Commonly Used Functions . . . . . . . . . . . . . . . 126

C.2 m-file Code for the Thermo CRS A465 . . . . . . . . . . . . . 134

C.3 m-file Code for the 7 DOF WAM . . . . . . . . . . . . . . 144

Appendix D CRS Functions in Python Code 150

Appendix E OpenCV Written in Python for Camera Calibration 153

$\begin{array}{ll}\text { List of References } & 108\end{array}$ 


\section{List of Tables}

4.1 The DH parameters for the Thermo CRS A465. . . . . . . . 50

4.2 The DH parameters for the 7 DOF WAM Arm. . . . . . . . 51

4.3 Absolute measurement concept results using DH convention. . . . . . 54

4.4 Absolute measurement concept results using Hayati convention. . . . 54

4.5 Relative measurement concept results. . . . . . . . . . . 56

4.6 AMC using DH with the presence of both input and output noise. . . 65

4.7 AMC using MDH with the presence of both input and output noise. . 65

4.8 Absolute measurement concept results using straight line trajectories. $\quad 69$

4.9 Relative measurement concept results using straight line trajectories. $\quad 70$

4.10 The mean results for straight line trajectories. . . . . . . . . 71

4.11 Absolute measurement concept results using planar trajectories. . . . 73

4.12 Relative measurement concept results using planar trajectories. . . . 73

4.13 The mean results for planar trajectories. . . . . . . . . . . 74

4.14 Absolute measurement concept results using volumetric trajectories. . 76

4.15 Relative measurement concept results using volumetric trajectories. . 76

4.16 The mean results for volumetric trajectories. . . . . . . . . . 77

5.1 Camera calibration results of the camera internal properties. . . . . 83

5.2 Experimental results for the relative measurement concept. . . . . . . 87

5.3 Experimental results for the relative measurement concept. . . . . . . 93 
5.4 Experimental results for the absolute measurement concept. . . . . . 96 


\section{List of Figures}

1.1 (a) The Thermo CRS A465, and (b) the 7 DOF WAM Arm from Barrett Technology Inc.. . . . . . . . . . . . . . . . . . . . . 3

2.1 Exaggerated absolute pose errors. . . . . . . . . . . . . . . . . 7

2.2 Exaggerated relative position errors. . . . . . . . . . . . 8

2.3 Illustration of single theodolite approach. . . . . . . . . . . . . . 14

2.4 Illustration of camera geometry. . . . . . . . . . . . . . . . . 16

2.5 Kinematic frame allocation: DH parameters . . . . . . . . . . . 19

2.6 Kinematic Frame Allocation including the Hayati parameters. . . . . 21

2.7 Illustration of a simple gradient descent problem. . . . . . . . . . . 23

3.1 Establishing the pose of $\Sigma_{b}$ relative to $\Sigma_{w} \ldots \ldots \ldots$

3.2 Establishing the pose of $\Sigma_{n+1}$ relative to $\Sigma_{n-1} \ldots \ldots \ldots$

4.1 The DH parameter assignment for the CRS A465 shown in its zero position. . . . . . . . . . . . . . . . 51

4.2 The DH parameters assignment of the WAM Arm shown in its zero position. .............................. 52

4.3 The (a) absolute accuracy and (b) relative accuracy after AMC calibration. The corresponding first 15 absolute and relative positions are shown in (c) and (d), respectively. . . . . . . . . . . . . 55 
4.4 The (a) absolute accuracy and (b) relative accuracy after RMC calibration. The corresponding first 15 absolute and relative positions are shown in $(\mathrm{c})$ and $(\mathrm{d})$, respectively. . . . . . . . . . . . 57

4.5 Simulation including only the input noise. The absolute accuracy (a) before and (b) after AMC calibration. The first 15 absolute position before and after AMC calibration are shown in (c) and (d), respectively. 60

4.6 Simulation including only the output measurement noise. The absolute accuracy (a) before and (b) after AMC calibration. The first 15 absolute positions before and after AMC calibration are shown in (c)

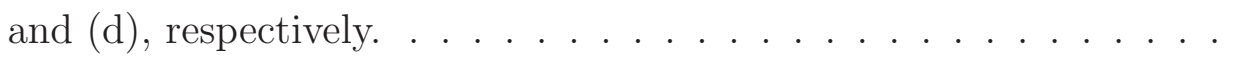

4.7 Simulations including both the input and output measurement noise. The absolute accuracy (a) before and (b) after calibration. The first 15 absolute poses before and after calibration are shown in (c) and (d), respectively. . . . . . . . . . . . . . . . . . . 63

4.8 The number of poses verses the condition number of the Jacobian. . . 66

4.9 Six varieties of straight line trajectories of end-effector pose. . . . . 68

4.10 Three varieties of planar trajectories of end-effector pose. . . . . . . 72

4.11 Four varieties of volumetric trajectories of end-effector pose. . . . . 75

5.1 Illustration of the $7 \times 10$ camera calibration board. . . . . . . . 80

5.2 Illustration of the camera calibration process. . . . . . . . . . . 83

5.3 Relative measurement setup for a Thermo CRS A465. . . . . . . . 84

5.4 (a) The computed A465 poses using the recorded joint angles, giving the nominal model. (b) The extrinsic parameters obtained using OpenCV, giving the measured model. . . . . . . . . . . 86

5.5 The relative pose of the nominal and measured models. . . . . . . . 86

5.6 Relative measurement setup for a WAM Arm. . . . . . . . . . . 88 
5.7 The kinematic model of the WAM in simulation. . . . . . . . . . . . 89

5.8 The kinematic model of the WAM in the experiment. . . . . . . . 93

5.9 The (a) absolute and (b) relative accuracies of the WAM Arm before calibration. The first 15 (c) absolute and (d) relative poses of the WAM Arm before calibration. . . . . . . . . . . . . . . .

5.10 The (a) absolute and (b) relative accuracies of the WAM after RMC. The first 15 (c) absolute and (d) relative poses of the WAM after RMC. 95

5.11 The (a) absolute and (b) relative accuracies of the WAM after AMC. The first 15 (c) absolute and (d) relative poses of the WAM after AMC. 97

A.1 Coordinate system representation of a rigid body . . . . . . . . . 108

A.2 Coordinate system representation of a point . . . . . . . . 116

A.3 Transformations between coordinate frames. (a) Pure translation, and pure rotation about the corresponding axes (b) $\mathbf{x}$-axis, (b) $\mathbf{y}$-axis and (d) z-axis . . . . . . . . . . . . . . . . . . . . . . . . . . 118

B.1 Pose representation of generic link $i$ of a manipulator. . . . . . . . . 123 


\section{Chapter 1}

\section{Introduction}

\subsection{Motivation}

Robot manipulator calibration has been an active research topic for many years. It is highly essential for robot manufacturing systems, because manipulators generally have vastly superior repeatability compared to their accuracy, which can render them unfeasible for some applications. Repeatability is the ability of the robot to return to the same taught end-effector position and orientation (pose) where the joint angles are taught to the robot and stored in the controller, while accuracy is a measure of how well the robot controller can place the end-effector in a pose where the required robot joint angles must be computed by the kinematic model embedded in the controller. The goal of robot kinematic calibration is to identify and compensate errors in the kinematic model so that the accuracy can be improved up to the limit represented by the repeatability.

The manipulator pose accuracy can be affected by geometric and non-geometric

errors [1]. Geometric errors are artifacts of the joint offsets and the errors in the nominal kinematic parameters. Other non-geometric errors include friction, inertia, 
applied load, flexibility, and temperature induced dimensional deformation. Experimental results reported in [1] conclude that about $95 \%$ of manipulator inaccuracies are due to the geometric errors. This is the motivation for this thesis to be focused on the development of a novel method to measure and identify the errors in the kinematic geometry of wrist-partitioned serial robot manipulators.

\subsection{Objectives}

The primary objective of this thesis is to develop a low-cost kinematic calibration method for serial manipulators using the relative measurement concept (RMC). Many existing kinematic calibration methods in the literature have used the absolute measurement concept (AMC). They can be expensive to perform due to the requirement of expensive equipment to accurately measure the manipulator pose. The measurement devices used for the required measurements are typically a coordinate measuring machine (CMM), laser interferometers, and laser theodolites, which can cost roughly US\$50,000to US\$100,000 per unit depending on the desired level of accuracy. The cost for an RMC system should be an order of magnitude less than an AMC system.

Several other objectives are:

- To refine and verify the validity of the RMC by creating a simulation where the errors in the kinematic parameters are specified first, and then identified using the algorithm developed in this thesis. A companion simulation based on the AMC was developed for comparison with the RMC.

- Validate the RMC experimentally with the apparatus yielding low cost, time efficient, and useful results. Unlike the work in the past references, in this work only a single camera is used together with an identifiable object (target) attached to the end-effector. Figure 1.1 shows images of the two manipulators 
used for the experiments reported in this thesis. The overall cost of the experimental setup, excluding the cost of the manipulator, is roughly $\$ 50$ to $\$ 100$.

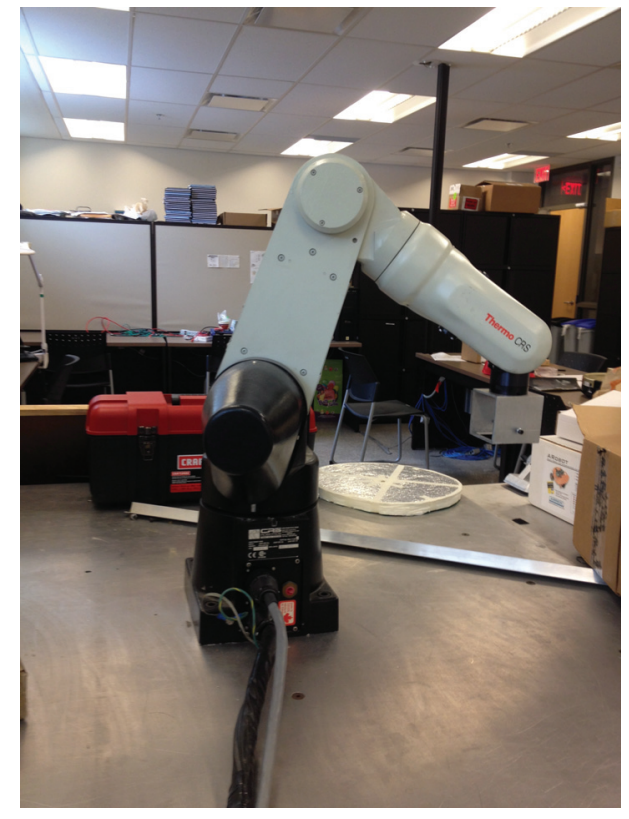

(a)

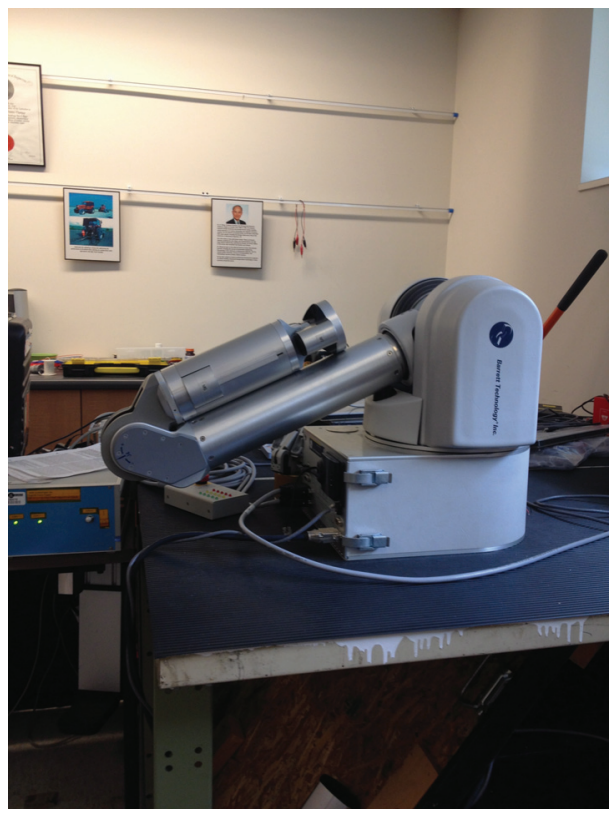

(b)

Figure 1.1: (a) The Thermo CRS A465, and (b) the 7 DOF WAM Arm from Barrett Technology Inc..

\subsection{Scope and Contributions to Knowledge}

The structure of this thesis is intended to meet all the objectives presented previously, and is divided into the following three main areas: (a) developing the kinematic model based on the serial manipulators being calibrated; (b) developing the kinematic calibration algorithm using simulations, and investigate the effects influencing the accuracy of the calibration results; and (c) developing the experimental procedure and setting up the camera calibration algorithm for the end-effector pose measurements 
used. The first area described above involves selecting a useful kinematic model for calibration based on the kinematic geometry of the robot. The second area contains the most important contributions of this thesis. They include:

- The derivation of the kinematic error model using relative errors.

- A genetic algorithm was developed to determine the initial guesses, such that the nonlinear optimization techniques used can converge to a global minimum.

- Performed simulations for analyzing how measurement noise, number of poses, and pose selection affect the numerical solution. This ensured the success of the experiment.

Finally, the third area is mainly relying on the camera calibration algorithm developed using OpenCV.

\subsection{Overview}

The remainder of the thesis is organized as follows. Chapter 2 presents some background information and literature review regarding the development of algorithms and the measurement setup used in this thesis. Detailed explanations are provided for all topics needed as the stepping stone to complete the goals of this thesis along with references to past research.

Chapter 3 investigates developing a feasible kinematic model representing the serial manipulator being calibrated. This includes a discussion of the properties of the kinematic model well suited to the calibration, as well as different conventions for parameterizing manipulator kinematics. Finally, the derivation of a differential kinematic model for calibration is reported. 
Chapter 4 reports the simulation results for the AMC and RMC calibration methods. External effects that could affect the numerical calibration results are summarized and discussed, such as measurement noise, the number of poses, and the pose trajectory selection.

Chapter 5 outlines the experimental setups and calibration results for both the Thermo CRS A465 and The WAM Arm from Barrett Technology Inc. The work here includes the method of measurement acquisition, camera calibration, experimental setup, procedure, and results.

Chapter 6 summarizes all the achievements made in this thesis. Conclusions stating the advantages and disadvantages for both the AMC and the RMC methods of kinematic calibration are presented. Finally, recommendations for future work on improving and fully taking advantage of the RMC are suggested. 


\section{Chapter 2}

\section{Background and Literature Review}

\subsection{Under the Hood of Kinematic Calibration}

It will be helpful at this point to imagine two geometrically similar but not identical robots, Model $A$ and Model $B$. Model $A$ and Model $B$ describes the geometry of the actual robot the embedded model in the robot controller, respectively. The parameters $\boldsymbol{x}_{A}$ and $\boldsymbol{x}_{B}$ represent the end-effector poses of the Model $A$ and Model $B$. The aim of the kinematic calibration is to improve the error between $\boldsymbol{x}_{A}$ and $\boldsymbol{x}_{B}$. The most common approach is to approximate $\boldsymbol{x}_{A}$ using Taylor series expansion about $\boldsymbol{x}_{n}$ (the computed pose using the nominal kinematic parameters, $\boldsymbol{\zeta}_{n}$ ) [2],

$$
\boldsymbol{x}_{A} \approx \boldsymbol{x}_{B}=\left[\begin{array}{ll}
\mathbf{p}_{B} & \boldsymbol{\Phi}_{B}
\end{array}\right]^{T}=\boldsymbol{x}_{n}+\delta \boldsymbol{x}=\boldsymbol{f}\left(\boldsymbol{\zeta}_{n}\right)+\frac{\partial \boldsymbol{f}}{\partial \boldsymbol{\zeta}} \delta \boldsymbol{\zeta}+\text { higher order terms }
$$

where $\mathbf{p}$ represents the end-effector position, $\boldsymbol{\Phi}$ represents the end-effector orientation (it can be expressed in many form of representations, and many of them are reviewed in Appendix A), and $\delta \zeta$ represents the errors in the kinematic parameters. Because each component of a pose is linearly independent, some literature approached the kinematic calibration problem by partial pose measurements only. 
The goal of the kinematic calibration is to estimate the $\boldsymbol{\zeta}$ that minimizes the errors between $\boldsymbol{x}_{A}$ and $\boldsymbol{x}_{B}$. Because the functional relationship between $\boldsymbol{x}_{B}$ and $\boldsymbol{\zeta}$ is nonlinear, many measurements of $\boldsymbol{x}_{A}$ are needed to converge to a useful solution. $\boldsymbol{x}_{A}$ can be measured using either absolute or relative measurements $[4,5]$.

\section{Absolute Measurements}

Consider a manipulator that is commanded to move through a series of configurations. At configuration $i$, the poses, $\boldsymbol{x}_{A, i}$ and $\boldsymbol{x}_{B, i}$, are produced. $\boldsymbol{x}_{A, i}$ is obtained using a measurement system, which is expressed with respect to the metrology frame $\Sigma_{m}$. On the other hand, $\boldsymbol{x}_{B, i}$ is computed by the controller using the forward kinematic model, and it is expressed with respect to the manipulator base frame $\Sigma_{b}$.

In order to estimate the errors embedded in the two poses, both poses must be expressed in the same reference frame. A fixed world frame $\Sigma_{w}$ is introduced as the common reference for the two poses, then the errors relative to $\Sigma_{w}$ are

$$
{ }^{w} \mathbf{e}_{i}={ }^{w} \boldsymbol{x}_{A, i}-{ }^{w} \boldsymbol{x}_{B, i}=\left[\begin{array}{ll}
\Delta{ }^{w} \mathbf{p}_{i} & \Delta{ }^{w} \boldsymbol{\Phi}_{i}
\end{array}\right]^{T},
$$

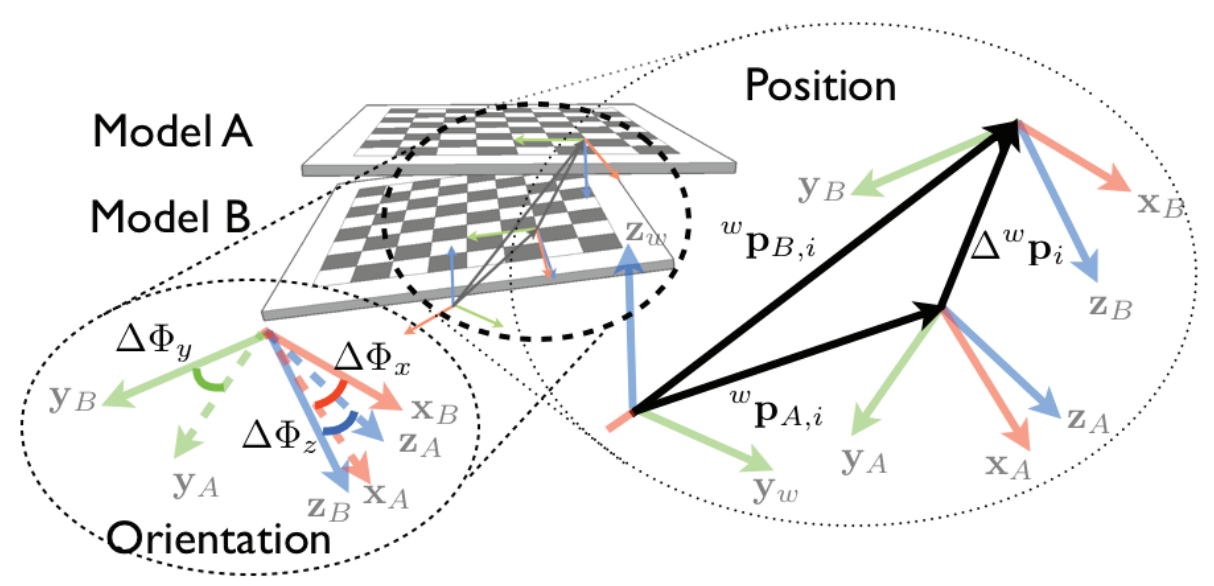

Figure 2.1: Exaggerated absolute pose errors. 
where ${ }^{w} \mathbf{e}_{i}$ is the absolute error (an array containing both position and orientation errors). The kinematic calibration performed using the absolute error is termed the absolute measurement concept or $A M C$. Figure 2.1 uses two chessboards as the conceptual representation of the measurement system to illustrate the absolute error.

\section{Relative Measurements}

Consider a manipulator that is commanded to move through the same series of configurations, where the configuration $j$ is next from $i$, producing two new poses, $\boldsymbol{x}_{A, j}$ and $\boldsymbol{x}_{B, j}$. Figure 2.2 uses two chessboards as the conceptual representation for illustrating relative position measurements only.

The end-effector poses, $\boldsymbol{x}_{A}$ and $\boldsymbol{x}_{B}$, are expressed relative to a common reference frame, $\Sigma_{w}$. The end-effector position $j$ relative to $i$ can be obtained from the vector

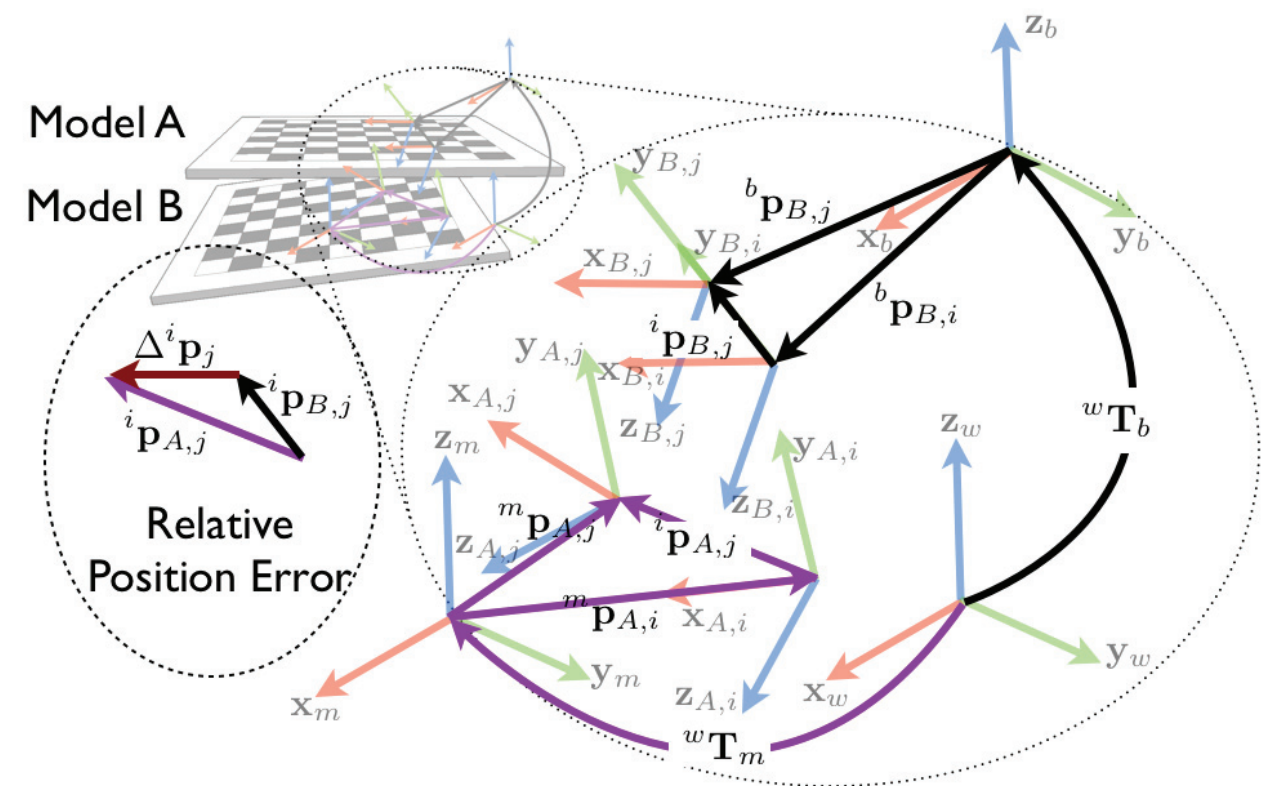

Figure 2.2: Exaggerated relative position errors. 
difference,

$$
\begin{gathered}
{ }^{i} \mathbf{p}_{A, j}={ }^{w} \mathbf{p}_{A, j}-{ }^{w} \mathbf{p}_{A, i}, \\
{ }^{i} \mathbf{p}_{B, j}={ }^{w} \mathbf{p}_{B, j}-{ }^{w} \mathbf{p}_{B, i} .
\end{gathered}
$$

The relative position errors are the errors embedded in the two relative positions,

$$
\Delta^{i} \mathbf{p}_{j}={ }^{i} \mathbf{p}_{A, j}-{ }^{i} \mathbf{p}_{B, j} .
$$

From [6], the relative orientation errors can be derived similarly to the relative position errors. The relative orientation errors are embedded in the two relative orientations,

$$
\Delta^{i} \boldsymbol{\Phi}_{j}={ }^{i} \boldsymbol{\Phi}_{A, j}-{ }^{i} \boldsymbol{\Phi}_{B, j} .
$$

Similar to the absolute error, both pose errors for robots A and B must also be expressed in the same reference frame. Because both Eqs. (2.4) and (2.5) are expressed relative to $i$, then the errors are

$$
{ }^{i} \mathbf{e}_{j}={ }^{i} \boldsymbol{x}_{A, j}-{ }^{i} \boldsymbol{x}_{B, j}=\left[\begin{array}{ll}
\Delta^{i} \mathbf{p}_{j} & \Delta^{i} \boldsymbol{\Phi}_{j}
\end{array}\right]^{T},
$$

where ${ }^{i} \mathbf{e}_{j}$ is the relative error (also an array containing both position and orientation errors). The kinematic calibration using the relative error is termed relative measurement concept or $R M C$ [6]. 


\subsection{Literature Review of Kinematic Calibration of 6R Serial Manipulators}

Improving the pose accuracy of a robot manipulator using kinematic calibration has been an active research topic [7] for nearly half a century. Two types of manipulators are generally used in industry now: serial and parallel manipulators. For each type of manipulator, each joint connecting two links together can either have a rotary degree-of-freedom (DOF) (revolute joint or R-pairs) or a linear DOF (prismatic joint

or P-pairs). The kinematic calibration performed in this thesis focuses exclusively on the R-pairs serial manipulators. In this section, the current state of the art of kinematic calibration research is reviewed.

\subsubsection{Kinematic Calibration Method}

A wide variety of different kinematic calibration methods have been developed over the past few decades, several relevant approaches are described next.

\section{Relative Measurement}

The work devised by Hayes and O'Leary [3] was the first literature found on the kinematic calibration method using relative position measurements. In this work, the calibration method was attempted on the KUKA KR-15/2, which is a $6 \mathrm{R}$ serial robot. The pose measurement here was achieved by having the robot draw lines in the $\mathbf{x}$ and $\mathbf{y}$ directions relative to the robot base frame along two straight edges. A CCD camera and an MEL displacement sesors were attached to the end-effector. The relative position measurements of the $\mathbf{x}$ and $\mathbf{y}$ component were embedded in the images taken from the CCD camera, and the $\mathbf{z}$ component was given by the MEL distance sensors. However, at this point of the work, only the simulation attempts 
were made for the calibration, and the results were not successful, as they always encountered rank deficiency of $\mathbf{J}$. The cause of this rank deficient $\mathbf{J}$ and the approach to overcome this issue is addressed later in this thesis.

Simpson and Hayes [4] continued the work from [3], which instead attached only a CCD camera on the end-effector of the Thermo CRS A465. The robot was commanded to move the end-effector in increments along the length of the precision-ruled straight edge with major increments of $1 \mathrm{~cm}$. The straight edge is placed carefully such that the first increment is aligned with the centre of the image taken by the CCD camera. For every $1 \mathrm{~cm}$ increment that the end-effector is moved, the observed misalignment between the centre of the image to the incremental line on the straight edge can be extracted as relative position error. In this work, calibration was attempted using both the simulated data and the experimental data. Like [3], the simulation attempted here were also not successful, as the rank deficienct $\mathbf{J}$ still appeared. The experiment was setup using only the CCD camera attached to the end-effector, resulting in only two position components ( $\mathbf{x}$ and $\mathbf{y}$ ) measured. The experimental result showed a solution that diverges away from the nominal parameters. The cost of this setup were reported to be roughly $\$ 6000$, which is about 100 times more expensive than the setup presented in this thesis.

Ha [5] on the other hand, approached relative position measurement a little differently. Instead of relying on the correct placement of measurement tools, Ha developed a suitable kinematic error model for recognizing the relative position errors. The experiment consists of a six DOF manipulator (MOTOMAN UP 20), a laser displacement sensor (accuracy of $\pm 0.01 \mathrm{~mm}$ ) and a machined grid plate (accuracy of $\pm 0.1 \mathrm{~mm})$. In this work, it was concluded that the relative measurement concept can be performed without the knowledge of the robot base. However, in this thesis, it shows that the orientation of the robot base is still needed. 


\section{Inverse Calibration Method}

Inverse calibration was developed to determine the errors observed in the end-effector poses, and use them to estimate the required joint angles to compensate for the errors [8-11]. With this method, no attempts on altering the manipulator model are made, but rather approximation functions for the end-effector errors are needed. Therefore, this method can also be referred to as the non-parametric accuracy compensation [12]. The approximation functions have no physical significance, but they consist of the information on both the geometric and non-geometric errors.

For a six DOF manipulator, the approximation functions are defined using a multivariate polynomial with six variables, which is quite complex. Shamma and Whitney [8] developed a method for six DOF wrist-partitioned manipulators by separating into two parts: the calibration of the shoulder and the wrist. This simplifies the approximation functions of the multivariate polynomial into two trivariate polynomials. Doria et al. [9] defined multiple second order spline functions, piecewise polynomials, to approximate the multivariate polynomials. Zhong et al. [10], on the other hand, applied a feed-forward artificial neural network to estimate the approximation functions.

Dolinsky [11] examined all the inverse calibration methods and observed that numerical estimation techniques can be compromised by numerical instability. Dolinsky then proposed a method for determining the approximation functions with genetic programming, which uses stochastics to symbolically generate the functions.

The main disadvantage for the non-parametric compensation technique [12] is that the robots can only be calibrated over a small volume of workspace in order to get a useful solution, which implies that if the robot is operating outside of this workspace, then some errors are going to surface. The calibration method used in this thesis is capable of calibrating the robot over a large volume of workspace. 


\section{Circle Point Analysis}

This method estimates the line equations of robot revolute joint axes for an arbitrary robot configuration by estimating the circle drawn out by each robot joint motion [13]. Because a least-squares technique is used to estimate each circle, more than three points are required. However, since the trajectory of the end-effector is not, in general, a circle if multiple joints are in motion simultaneously, the robot must be moved one joint at a time.

The true kinematic model parameters can be extracted from the line equations defining the joint axes. Two strategies have been developed to extract the kinematic parameters from the identified joint axes. The first, Stone [13], consists of deriving an analytical formula for the kinematic parameters in terms of the link homogeneous transformation matrices from the S-model. The second, Sklar [14,15], consists of computing common normal lengths, offset distances, and twist angles directly from the identified line equations using standard vector algebra relationships and the existing geometrical constraints.

Kim et al. [16] reviewed the work from Stone and Sklar on the circle point analysis, and they conducted an experiment using a HYUNDAI robot AE 7601 and KIMI-tester (a type of CMM). The experiment showed that the circle point analysis is capable of improving the absolute positioning accuracy of the robot by an order of magnitude, from standard deviations of $10 \mathrm{~mm}$ to $1 \mathrm{~mm}$ over the entire manipulator workspace.

Several disadvantages are found in the circle point analysis [12]. This method requires the robot to move one joint at a time, and sufficient number of measurement points must be made to obtain a useful solution. The calibration method used in this thesis allows all the robot joints to be moved simultaneously, and at least 40 measurement points are needed to yield an acceptable solution. 


\subsubsection{Measurement Method}

There are many papers in the literature reporting excellent kinematic calibration results, where measurements are made with a CMM. However, they will not be mentioned here because a CMM is extremely expensive to use for measuring just the position components with the required accuracy.

\section{Theodolites}

A theodolite is simply a telescope that has been instrumented such that the line of sight is precisely known. This line of sight is established by two angles, one angle is on the horizontal plane and the other is on the vertical plane. A theodolite is used by pointing the telescope to the target (attached to the end-effector), $\Sigma_{t}$, and using the two angles established to calculate a scaled location of $\Sigma_{t}$ relative to the theodolite, as shown in Figure 2.3. The wireframe image of the theodolite is taken from [12].

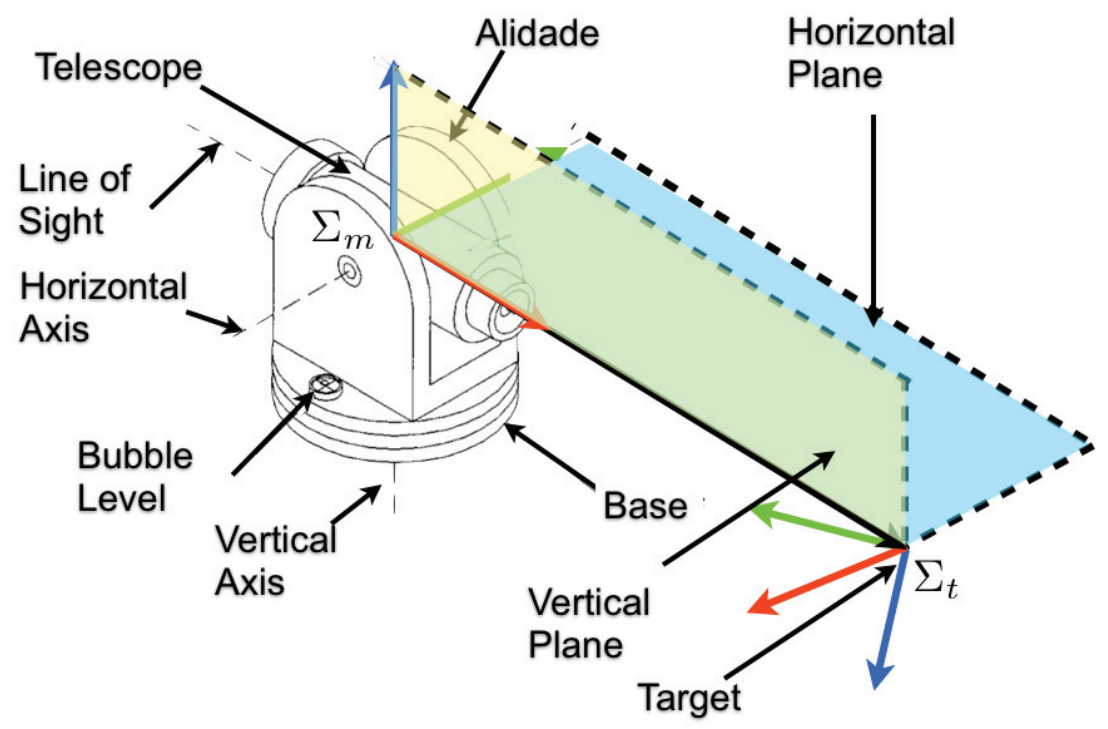

Figure 2.3: Illustration of single theodolite approach. 
Whitney, Lozinski, and Rourke [18] used a single theodolite measurement system to perform kinematic calibration of a PUMA 560 manipulator. Whitney reported in [18] that it was time consuming to find the target and difficult to centre the cross-hairs accurately. Judd and Knasinski [1] calibrated an Automatix AID900 using triangulation between two theodolites. Chen and Chao [19], on the other hand, approached the calibration of a PUMA 560 using triangulation between three theodolites. Accurate relative pose information between multiple theodolites is required for triangulation, but the process of determining such information was not reported $[12,20]$.

Although theodolite is a common tool used as the measurement device for many kinematic calibration in the past, it is incapable of measuring the orientation component of the robot end-effector. In this thesis, a single camera is used as the measurement device, and it is capable of determining the full pose information about the end-effector.

\section{Vision-Based Devices}

A vision-based device, or a camera, can be used to extract the full pose information of a target object, $\Sigma_{t}$, relative to the camera. A camera placed in the 3D Euclidean $\left(E_{3}\right)$ space views the target also in $E_{3}$, but the camera can only project the view of the target to the image space, as illustrated in Figure 2.4. This means that the full pose information can be obtained by converting the view in the image space back to the $E_{3}$ space. This procedure is done as part of camera calibration process.

Zhuang, Wu, and Roth [21] performed kinematic calibration on a 4 DOF Intellidex robot by attaching a charge-coupled device (CCD) camera with a $25 \mathrm{~mm}$ lens to the end-effector viewing an identifiable target. They developed a camera calibration 

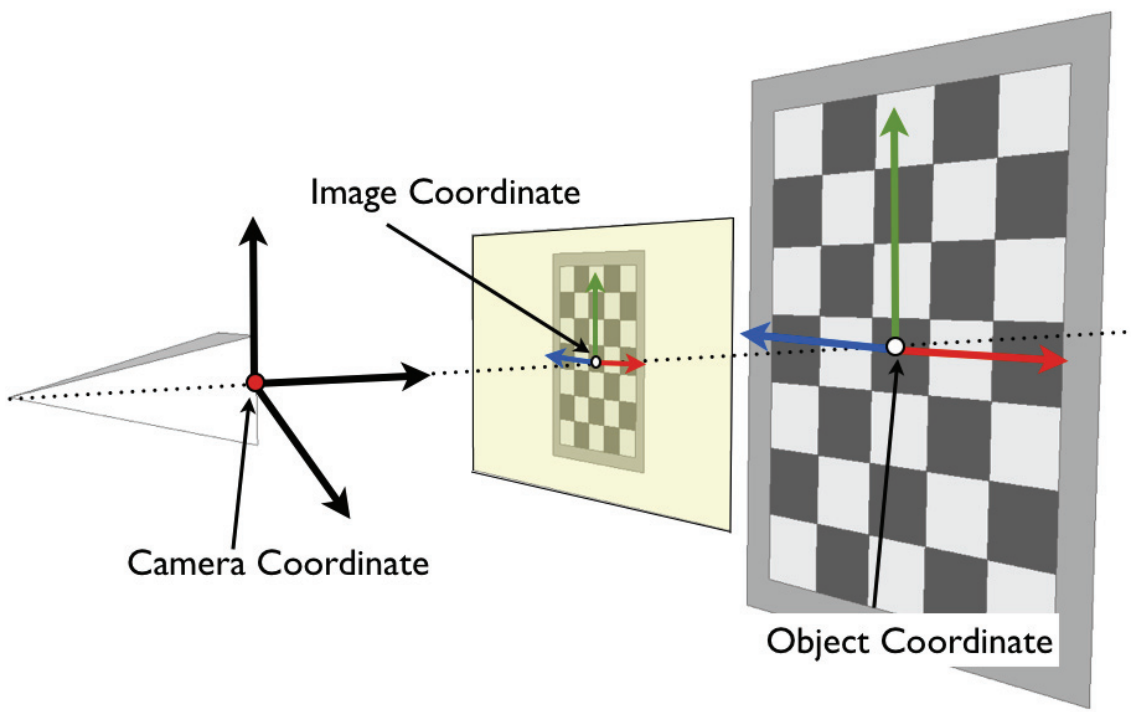

Figure 2.4: Illustration of camera geometry.

algorithm for computing the full pose information of the target relative to the camera, and then in return extract the full pose of the end-effector.

Zhuang and Roth later reported with Xu and Wang [22] that the camera calibration algorithm used in [21] can be quite computationally intensive. This is because that the camera itself must be recalibrated each time the manipulator is configured. In this paper, Zhuang et al proposed a camera calibration modified from Tsai's radial alignment constraint (RAC) [23]. It was concluded that using the modified RAC method produced more accurate results than the original RAC method, and the computational time did not increase much.

Xiong [24] set up stereo vision as the measurement system for the calibration of a microrobotic system, which is essentially triangulating between two cameras. This setup is quicker at obtaining full pose information, because it does not require a camera calibration on every image.

In this thesis, the camera calibration algorithm used is based on the one written in OpenCV. The comparison on the identified accuracy and the time elapsed in the 
calibration process between the OpenCV algorithm and the modified RAC algorithm were not made. Therefore, a conclusive comparison between the two cannot be made.

\section{Telescoping Ballbar System}

The telescoping ballbar system is a machine-precisioned bar with ball-like structure at each end. The balls of the sensor are kinematically located in the magnetic cups (one is placed as the reference while the other is placed on the point of interest, i.e. the end-effector of a manipulator in the case of kinematic calibration). This arrangement allows the ballbar to measure small variations in the radius of a circular path that the end-effector is programmed to follow.

Nubiola, Slamani, and Bonev [25] proposed a relatively low cost $(\$ 13,000.00)$ but high accuracy $( \pm 0.003 \mathrm{~mm})$ measurement system, and the technique for measuring a set of more than 50 distinct poses of a manipulator using Renishaw's QC20-W telescoping ballbar standard kit. The measurement system involves buying additional components from Renishaw's to build on top of the standard kit into the form of a hexapod (Gough-Stewart platform). All possible poses that the manipulator endeffector can make are obtained by solving the forward kinematics of the hexapod. An experiment using this particular technique was tested on an ABB IRB 120 industrial robot, and the result is presented in [25].

The telescoping ballbar system is a contact-based measurement method, implying that the movement of the robot end-effector is restricted by the movement of the ballbar system. By using a camera as the measurement device, a wider range of endeffector movement is allowed during the measurement phase. Later in the thesis, it shows that a larger workspace for measurement, can lead to a more accurate identified solution. 


\subsubsection{Kinematic Modelling}

The standard model for the kinematic geometry of serial manipulators is the DenavitHartenburg (DH) convention. Several researchers $[2,4]$ have performed kinematic calibration using the DH convention, but most of them have reported a major drawback [26] (they found that there is discontinuity in the kinematic geometry when numerical algorithm is applied to calibrate a robot that has nominally parallel joint axes).

An alternate four-parameter model proposed by Hayati [27] addresses this particular issue. This convention can only be used to express the link with nominally parallel joint axes. A five-parameter model is proposed by Veitschegger and Wu [28], which builds on the convention in [27], but it can also be used on the link with nominally perpendicular joint axes. These two conventions are commonly used as the kinematic model for calibration, because they retain the simplicity of the DH convention.

There are other alternate models developed for kinematic calibration that are specifically for the sake of research, they are the S-model and the zero-referenced model. The S-model developed by Stone and Sanders [29] allows each coordinate system defined to be displaced an arbitrary distance and angle about the joint axis from the location described by the DH convention. The zero-referenced model developed by Mooring and Tang $[12,30]$ makes use of the general spatial rigid body displacement

equation (screw coordinate system) to develop the kinematic model. Both models are defined using six parameters, which is precisely the number of parameters required to completely define the pose between two arbitrary coordinate frames. The S-model and the zero-referenced model are less restrictive than all the other conventions. 


\section{Denavit-Hartenburg Convention}

The kinematic model of a manipulator is defined by attaching a coordinate frame, $\Sigma_{i}$, to each manipulator link $i$. Denavit and Hartenburg proposed a convention for describing the frame $\Sigma_{i}$ relative to $\Sigma_{i-1}$ with four independent parameters, the DenavitHartenburg (DH) parameters [31-33], as illustrated in Figure 2.5.

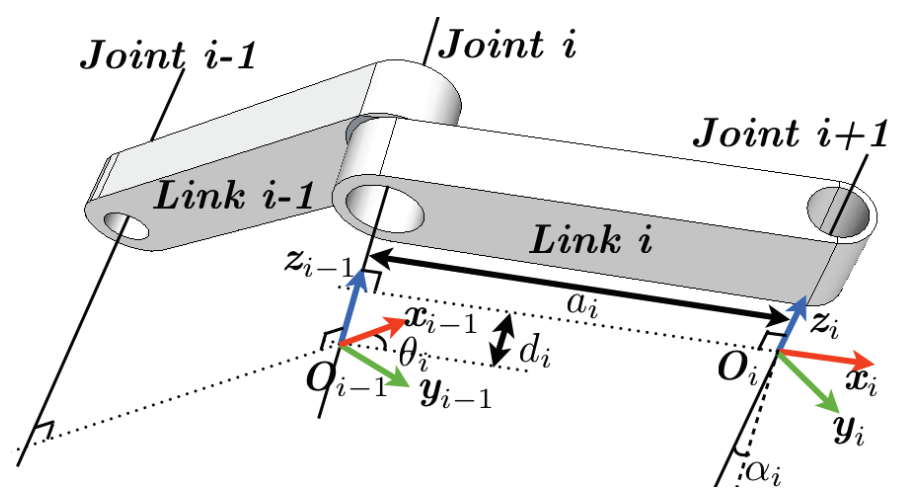

Figure 2.5: Kinematic frame allocation: DH parameters

Consider two lines in space representing neighbouring joint axes $\mathbf{z}_{i-1}$ and $\mathbf{z}_{i}$, which are typically skewed lines. There is a unique common normal whose direction $\overrightarrow{\mathbf{n}}_{i}$ is

$$
\overrightarrow{\mathbf{n}}_{i}=\frac{\mathbf{z}_{i-1} \times \mathbf{z}_{i}}{\left|\mathbf{z}_{i-1} \times \mathbf{z}_{i}\right|} .
$$

- The $\mathbf{x}_{i}$-axis is typically on or parallel to $\overrightarrow{\mathbf{n}}_{i}$, and points from $\mathbf{z}_{i-1}$-axis to $\mathbf{z}_{i}$-axis.

- The intersection of $\mathbf{x}_{i}$-axis and $\mathbf{z}_{i}$-axis defines the origin $O_{i}$ of $\Sigma_{i}$.

The following sequential transformations of coordinate frames are applied to transform the frame $\Sigma_{i-1}$ into $\Sigma_{i}$ :

- Rotate the frame $\Sigma_{i-1}$ about $\mathbf{z}_{i-1}$ by the joint angle, $\theta_{i}$.

- Translate the current $\Sigma_{i-1}$ along $\mathbf{z}_{i-1}$ by the link offset, $d_{i}$. 
- Translate the current $\Sigma_{i-1}$ along $\mathbf{x}_{i}$ by the link length, $a_{i}$.

- Rotate the current $\Sigma_{i-1}$ about $\mathbf{x}_{i}$ by the link twist, $\alpha_{i}$, to become $\Sigma_{i}$.

If the sequential transformations are in terms of the homogeneous transformation matrices (refer to Appendix A for more information), then the following transformation can be obtained using several elementary transformations:

$$
\begin{aligned}
{ }^{i-1} \mathbf{T}_{i}\left(\theta_{i}\right) & =\mathbf{T}_{\text {rot }}\left(\mathbf{z}_{i-1}, \theta_{i}\right) \mathbf{T}_{\text {trans }}\left(\mathbf{z}_{i-1}, d_{i}\right) \mathbf{T}_{\text {trans }}\left(\mathbf{x}_{i}, a_{i}\right) \mathbf{T}_{\text {rot }}\left(\mathbf{x}_{i}, \alpha_{i}\right), \\
& =\left[\begin{array}{cccc}
\cos \theta_{i} & -\sin \theta_{i} \cos \alpha_{i} & \sin \theta_{i} \sin \alpha_{i} & a_{i} \cos \theta_{i} \\
\sin \theta_{i} & \cos \theta_{i} \cos \alpha_{i} & -\cos \theta_{i} \sin \alpha_{i} & a_{i} \sin \theta_{i} \\
0 & \sin \alpha_{i} & \cos \alpha_{i} & d_{i} \\
0 & 0 & 0 & 1
\end{array}\right] .
\end{aligned}
$$

The pose of $\Sigma_{i}$ with respect to $\Sigma_{i-1}$ is embedded in ${ }^{i-1} \mathbf{T}_{i}\left(\theta_{i}\right)$.

Consider the case of two lines in space (neighbouring joint axes) that intersect, the common normal $\overrightarrow{\mathbf{n}}_{i}$ has zero length, and thus $\overrightarrow{\mathbf{n}}_{i}$ has to be defined differently. Assign $O_{i}$ to locate at the intersection of $\mathbf{z}_{i-1}$-axis and $\mathbf{z}_{i}$-axis. Then $\mathbf{x}_{i}$-axis is assigned to be on the line perpendicular to the plane spanned by the $\mathbf{z}_{i-1}$-axis and $\mathbf{z}_{i}$-axis.

Now consider the case of the two lines in space (neighbouring joint axes) that are parallel, there exist an infinite number of common normals with equal lengths. The common normal $\overrightarrow{\mathbf{n}}_{i}$ is commonly chosen as one of the two arbitrary choices below:

1. Choose the $\overrightarrow{\mathbf{n}}_{i}$ that intersects $\overrightarrow{\mathbf{n}}_{i-1}$, where $d_{i}=0[34]$.

2. Choose the $\overrightarrow{\mathbf{n}}_{i}$ that intersects $\overrightarrow{\mathbf{n}}_{i+1}$, where $d_{i+1}=0[35]$.

It is impossible in practice to manufacture a link with perfectly parallel joint axes. Because the joint axes that are nearly parallel can cause the common normal to 
actually be unique, resulting in a drastic length change for $\overrightarrow{\mathbf{n}}_{i}$. This discontinuity can cause numerical instability in the kinematic calibration process.

\section{Hayati Convention: Modified DH Convention}

Hayati proposed a four-parameter model in [27] to overcome the problem caused by nearly parallel joint axes. The model defines a plane perpendicular to the $\mathbf{z}_{i}$-axis in which $d_{i}=0$, as illustrated in Figure 2.6. Instead of relying on $\overrightarrow{\mathbf{n}}_{i}$, the $\mathbf{x}_{i}$-axis is defined as the directed line segment connecting the $\mathbf{z}_{i-1}$-axis and $\mathbf{z}_{i}$-axis in the plane.

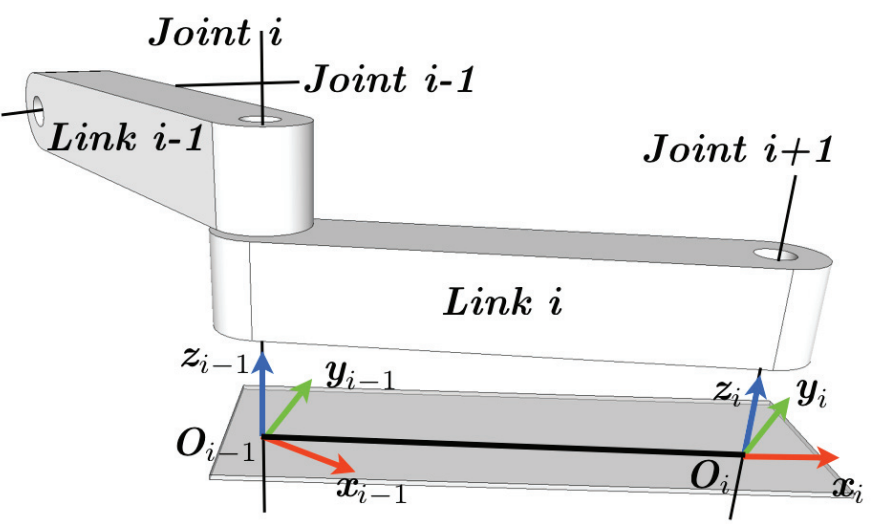

Figure 2.6: Kinematic Frame Allocation including the Hayati parameters.

The following sequential transformations of coordinate frames are applied to transform the frame $\Sigma_{i-1}$ into $\Sigma_{i}$ :

- Rotate the frame $\Sigma_{i-1}$ about $\mathbf{z}_{i-1}$ by the joint angle, $\theta_{i}$.

- Translate the current $\Sigma_{i-1}$ along $\mathbf{x}_{i}$ by the link length, $a_{i}$.

- Rotate the current $\Sigma_{i-1}$ about $\mathbf{x}_{i}$ by the link $\mathbf{x}$-twist, $\alpha_{i}$.

- Rotate the current $\Sigma_{i-1}$ about $\mathbf{y}_{i}$ by the link $\mathbf{y}$-twist, $\beta_{i}$, to become $\Sigma_{i}$. 
The sequential transformations can be applied in terms of homogeneous transformation matrices,

$$
\begin{aligned}
{ }^{i-1} \mathbf{T}_{i}\left(\theta_{i}\right) & =\mathbf{T}_{\text {rot }}\left(\mathbf{z}_{i-1}, \theta_{i}\right) \mathbf{T}_{\text {trans }}\left(\mathbf{x}_{i}, a_{i}\right) \mathbf{T}_{\text {rot }}\left(\mathbf{x}_{i}, \alpha_{i}\right) \mathbf{T}_{\text {rot }}\left(\mathbf{y}_{i}, \beta_{i}\right), \\
= & {\left[\begin{array}{cccc}
-s_{\theta_{i}} s_{\alpha_{i}} s_{\beta_{i}}+c_{\theta_{i}} c_{\beta_{i}} & -s_{\theta_{i}} c_{\beta_{i}} & s_{\theta_{i}} s_{\alpha_{i}} c_{\beta_{i}}+c_{\theta_{i}} s_{\beta_{i}} & a_{i} c_{\theta_{i}} \\
c_{\theta_{i}} s_{\alpha_{i}} s_{\beta_{i}}+s_{\theta_{i}} c_{\beta_{i}} & c_{\theta_{i}} c_{\beta_{i}} & -c_{\theta_{i}} s_{\alpha_{i}} c_{\beta_{i}}+s_{\theta_{i}} s_{\beta_{i}} & a_{i} s_{\theta_{i}} \\
-c_{\alpha_{i}} s_{\beta_{i}} & s_{\alpha_{i}} & c_{\alpha_{i}} c_{\beta_{i}} & 0 \\
0 & 0 & 0 & 1
\end{array}\right], }
\end{aligned}
$$

where $c$ and $s$ represent cos and sin, respectively, with the argument being any angular Hayati parameter, $\theta_{i}, \alpha_{i}$, or $\beta_{i}$.

There are many other kinematic modelling conventions intended for a wide variety of applications. The two just described are widely used in kinematic calibration, and they are chosen as the modelling convention used in this thesis.

\subsection{Identifying Kinematic Parameters}

The most common methods for identifying parameters are formulated as nonlinear optimization problems that minimize the errors between $\boldsymbol{x}_{A}$ and $\boldsymbol{x}_{B}$. Multiple techniques can be applied to obtain the solution for such problems, such as the nonlinear least-squares method, genetic algorithm, and singular value decomposition (SVD).

\subsubsection{Nonlinear Least-Squares: Gradient Based Search}

Most problems involving parameters identification are typically estimated by some form of least-squares. The simplest problems involves a system of linear equation, 
where linear least-squares is applied. For parameters that are nonlinear in their models (the case in most kinematic calibration problems) are often estimated using nonlinear least-squares, which typically involves linearizing a current estimate and apply linear least-squares iteratively until a solution is converged.

Consider a two-parameter $\left(\zeta_{1}\right.$ and $\left.\zeta_{2}\right)$ optimization problem, the objective function $L$ needs to be minimized. Figure 2.7 illustrates the concept behind the gradient descent with two search parameters and a quadratic objective function, $L=\|\mathbf{e}\|_{2}$, where $\mathbf{e}=\boldsymbol{x}_{A}-\boldsymbol{x}_{B}$. However, instead of a two-parameter search, the kinematic calibration of a $6 \mathrm{R}$ serial manipulator is at least a 24-parameter search problem. Nevertheless, the concept remains the same as the two-parameter problem.

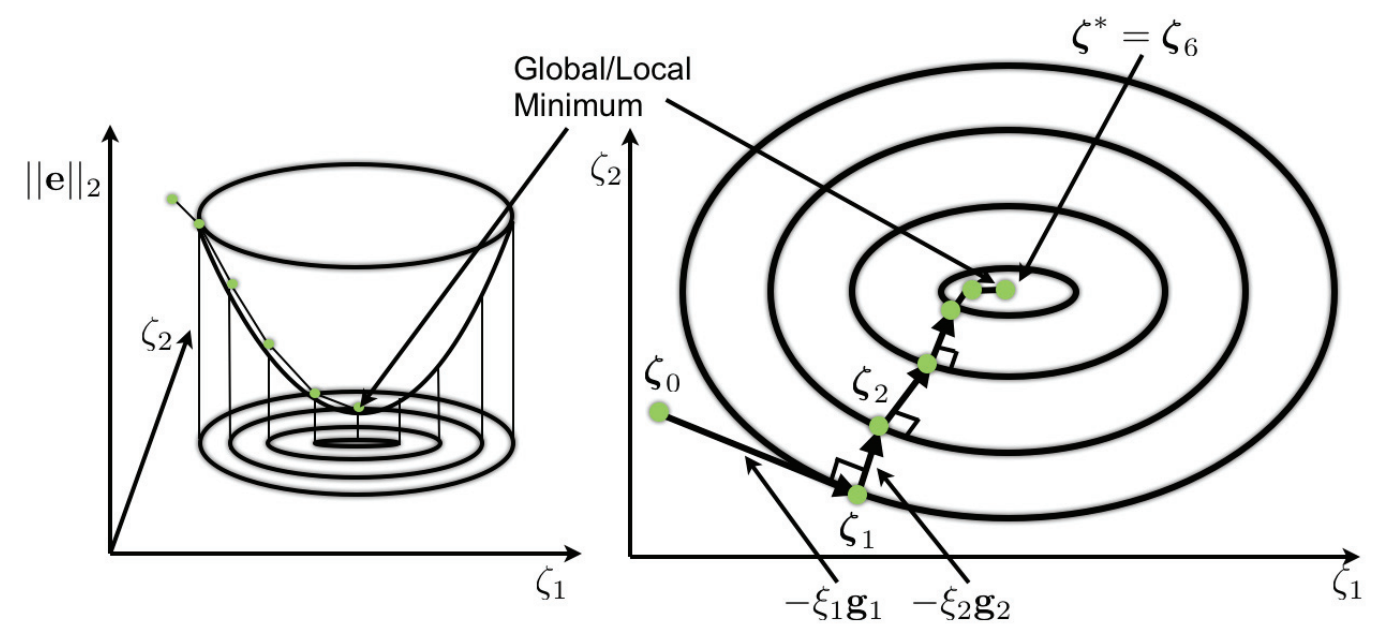

Figure 2.7: Illustration of a simple gradient descent problem. 
The solution for $\boldsymbol{\zeta}$ is estimated using the gradient information of the objective function. The optimal solution $\boldsymbol{\zeta}^{*}$ is found when $L$ is reduced iteratively to the minimum. The mathematics for gradient methods range from simple to highly sophisticated, the ones focused in this section are the steepest descent method [36,37], Newton method [36,38], Gauss-Newton method [12,36,38], and the Levenburg-Marquardt (LM) algorithm $[12,39-41]$.

\section{Steepest Descent Method}

Let $L_{k}$ denote the objective function evaulated at the $k^{\text {th }}$ iteration step. The basic requirement is to improve the estimate in each iteration, such that

$$
L_{k+1}<L_{k}
$$

By applying Taylor series expansion, the $L_{k+1}$ can be estimated as

$$
L_{k+1}=L_{k}+\delta L_{k} \approx L_{k}+\mathbf{g}_{k}^{T} \delta \boldsymbol{\zeta}_{k}
$$

where $\mathbf{g}_{k}$ is the gradient vector of $L_{k}$.

The gradient vector tells the algorithm the direction to search for the solution, even though it may not point towards the optimal solution in many practical cases [37]. By applying Eq. (2.11) to the inequality (2.10), the expression becomes

$$
\mathbf{g}_{k}^{T} \delta \zeta_{k}<0
$$

This direction of search for the optimal solution is the steepest descent. If the inequality (2.12) is positive instead, the search direction becomes the steepest ascent [37], then the estimated solution tends to diverge away from the correct solution. 
In the steepest descent method, the trajectory to the solution follows a zig-zag pattern as illustrated in Figure 2.7. The $\delta \boldsymbol{\zeta}_{k}$ in $L_{k}$ is

$$
\delta \zeta_{k}=-\xi \mathbf{g}_{k}
$$

where $\xi$ is a small positive constant that decreases the value of $L_{k}$. The gradient vector of the next iteration step is defined as

$$
\mathbf{g}_{k+1}=\nabla L\left(\boldsymbol{\zeta}_{k}+\delta \boldsymbol{\zeta}_{k}\right)
$$

The optimal solution $\delta \boldsymbol{\zeta}^{*}$ is found when $L$ is less than the minimum specified tolerance $\kappa$ and $\mathbf{g}^{*} \approx 0$.

In a kinematic calibration problem, the Jacobian matrix $\mathbf{J}$ is defined as the first derivative term of Eq. (2.1). Then, the gradient vector $\mathbf{g}_{k}$ can be computed to be

$$
\mathbf{g}_{k}=\frac{\partial \mathbf{e}^{T} \mathbf{e}}{\partial \boldsymbol{\zeta}_{k}}=2 \mathbf{e} \frac{\partial \mathbf{e}}{\partial \boldsymbol{\zeta}_{k}}=-2 \mathbf{J}_{k}^{T} \mathbf{e}
$$

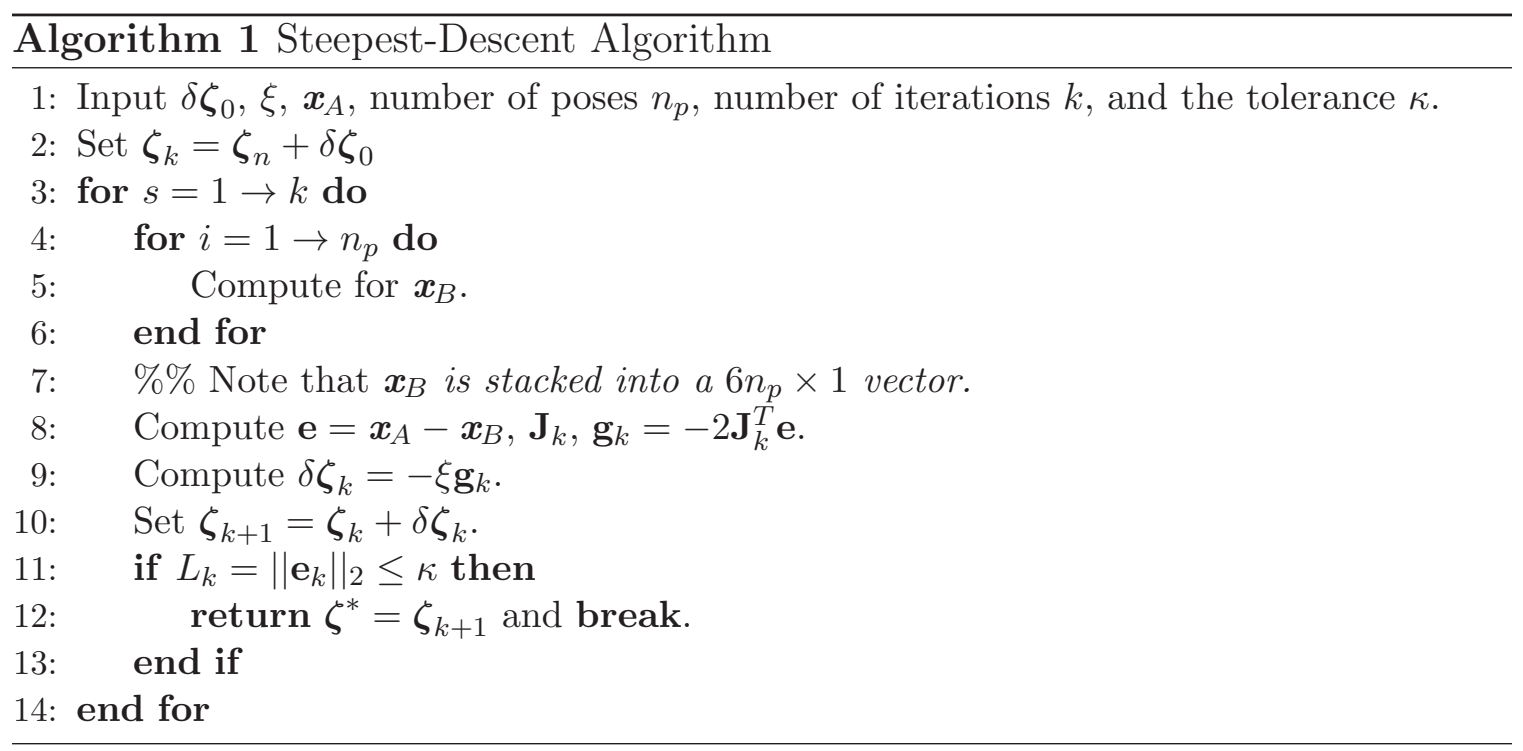




\section{Newton (Newton-Raphson) Method}

Now, consider taking the Taylor series expansion of the gradient vector in Eq. (2.14), $\mathbf{g}_{k+1}$ can then be approximated as

$$
\mathbf{g}_{k+1}=\mathbf{g}_{k}+\delta \mathbf{g}_{k} \approx \mathbf{g}_{k}+\mathbf{G}_{k} \delta \boldsymbol{\zeta}_{k}
$$

where $\mathbf{G}_{k}$ is the Hessian matrix of $L_{k}[12]$. For $L$ to be at the minimum, $\mathbf{g}$ has to be zero, and $\mathbf{G}$ must be positive definite. Therefore, $\mathbf{g}_{k+1}=0$ at such point, then Eq. (2.16) becomes

$$
\mathrm{G}_{k} \delta \zeta_{k}=-\mathrm{g}_{k}
$$

This updating strategy incorporating Eq. (2.17) and

$$
\zeta_{k+1}=\zeta_{k}+\xi \delta \zeta_{k}
$$

constitute the Newton method or Newton-Raphson method [12].

The Hessian matrix $\mathbf{G}_{k}$ is the gradient of $\mathbf{g}_{k}$, and it can be derived from the direct differentiation of Eq. (2.15), then

$$
\mathbf{G}_{k}=2 \mathbf{J}_{k}^{T} \mathbf{J}_{k}+2 \mathbf{S}_{k}
$$

where

$$
\mathbf{S}_{k}=\mathbf{e}_{k} \mathbf{H}_{k}=\mathbf{e}_{k} \frac{\partial^{2} \mathbf{e}_{k}}{\partial \zeta^{2}}
$$

With Eqs. (2.15) and (2.19) the Newton method becomes

$$
\left(\mathbf{J}_{k}^{T} \mathbf{J}_{k}+\mathbf{S}_{k}\right) \delta \boldsymbol{\zeta}_{k}=\mathbf{J}_{k}^{T} \mathbf{e}_{k}
$$


An algorithm for yielding an estimate of solution using the Newton method is not presented, because it is difficult to apply this method in most practical applications. It can be difficult to compute the second derivative term $\mathbf{S}$ for most practical problems. The $\mathbf{S}$ term is generally approximated somehow, which leads to the following two methods, the Gauss-Newton method and the Levenburg-Marquardt algorithm or LM algorithm.

\section{Gauss-Newton Method}

Eq. (2.19) can be difficult to compute due to the second derivative term $\mathbf{S}$ in the Hessian matrix G. There are two commonly used methods for reducing the complexity in the computation of $\mathbf{G}$. The first method is to ignore the $\mathbf{S}$ term, but this implies that the initial guess $\delta \boldsymbol{\zeta}_{0}$ is close to the correct solution, and the Newton method is reduced to the Gauss-Newton method,

$$
\mathbf{J}_{k}^{T} \mathbf{J}_{k} \delta \boldsymbol{\zeta}_{k}=\mathbf{J}_{k}^{T} \mathbf{e}_{k}
$$

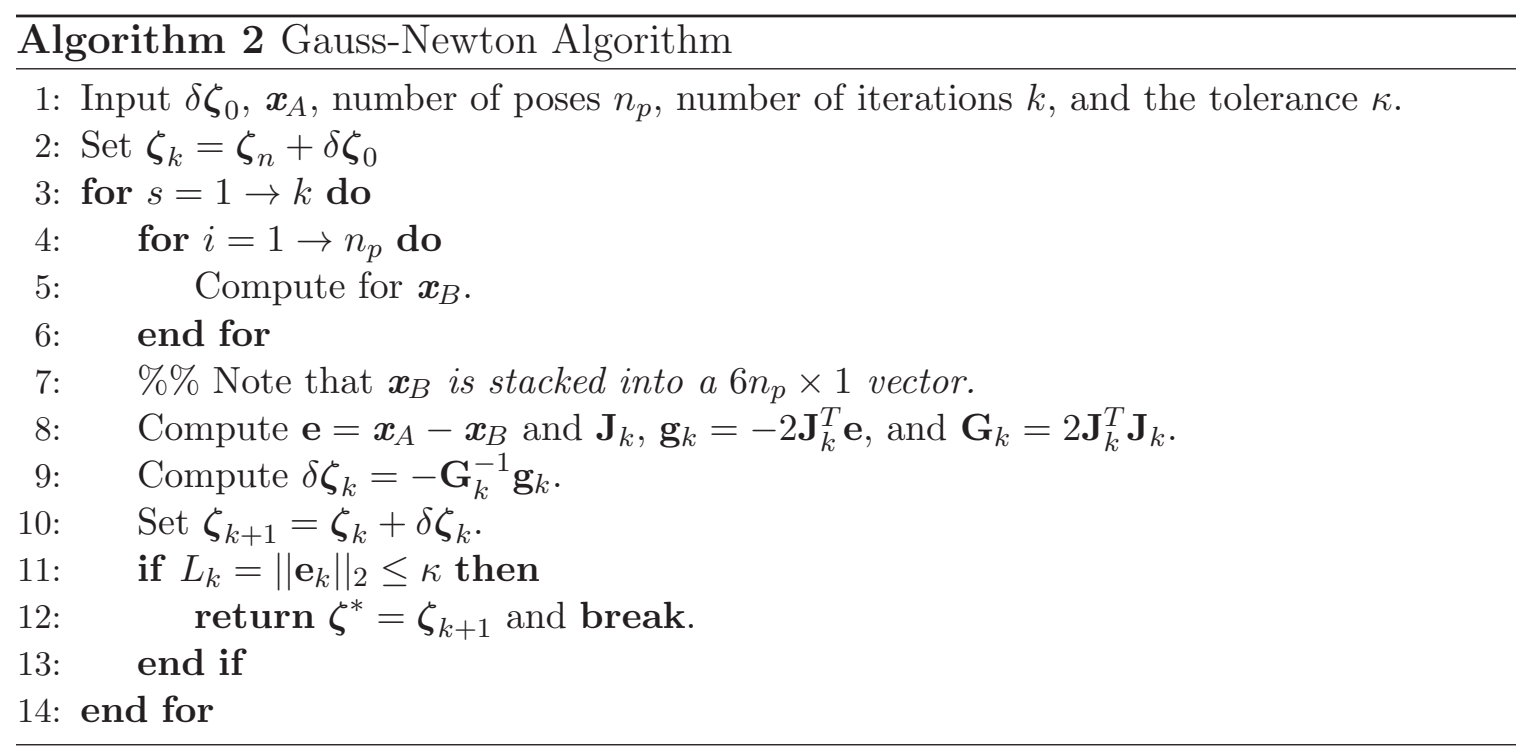


The Gauss-Newton method resembles the pseudoinverse solution of the linear least-squares algorithm. This method converges quickly if $\delta \boldsymbol{\zeta}_{0}$ is close to the correct solution or if $\mathbf{J}$ is nonsingular. Otherwise, the method breaks down and it may not converge at all.

\section{Levenburg-Marquardt (LM) Algorithm}

The second method to reduce the complexity of computing $\mathbf{G}$ is to replace $\mathbf{S}$ by a timevarying positive-definite matrix (a diagonal matrix governed by a scaling factor, $\mu_{k}$ ) [12]. This form of the Newton method is referred to as the Levenburg-Marquardt (LM) algorithm, which is designed to overcome the singularity issue in $\mathbf{J}$, while maintaining the convergence properties near optimal solution from the Gauss-Newton method,

$$
\left(\mathbf{J}_{k}^{T} \mathbf{J}_{k}+\mu_{k} \mathbf{I}\right) \delta \boldsymbol{\zeta}_{k}=\mathbf{J}_{k}^{T} \mathbf{e}_{k}
$$

where $\mathbf{I}$ is an identity matrix with the size of $\mathbf{J}^{T} \mathbf{J}$. As $\mu_{k} \rightarrow 0$, the LM algorithm has the convergence properties of Gauss-Newton. And as $\mu_{k} \rightarrow \infty$, the matrix $\left(\mathbf{J}^{T} \mathbf{J}+\mu \mathbf{I}\right)$ becomes positive-definite, then the LM algorithm has the convergence properties of the steepest descent. It has been shown that the LM algorithm is more robust in the search for optimal solutions compared to the Gauss-Newton method [36], and it is used in many practical applications.

There are many investigations on the possible methods for selecting $\mu_{k}$ at each iteration for the best approach to get an optimal solution. Two of them are:

Method 1 (Levenburg [40]): The value $\mu_{k}$ is selected using a line search to minimize the cost function $L$ using the solution estimated from Eq. (2.23), while any other parameters are held constant. However, this method is somewhat impractical, as its computation time is longer due to the need to search for $\mu_{k}$. 
Method 2 (Marquardt [41]): The value $\mu_{k}$ is initially set to some positive value $\left(\mu_{k}=0.01\right)$ and introduce a scaling factor of $v>1(v=10)$. The factor $v$ is used to increase or decrease $\mu_{k}$. In the first iteration, $\mu_{k}$ is reduced by a factor of $v$ to push the algorithm closer to Gauss-Newton. At each subsequent iteration $k, L_{k}$ is evaluated and compared against $L_{k-1} \cdot \mu_{k}$ is continuously increased by a factor of $v$ until $L_{k}<L_{k-1}$. Once $L_{k}<L_{k-1}$ is achieved, then $\mu_{k}$ can be decreased by a factor of $v$.

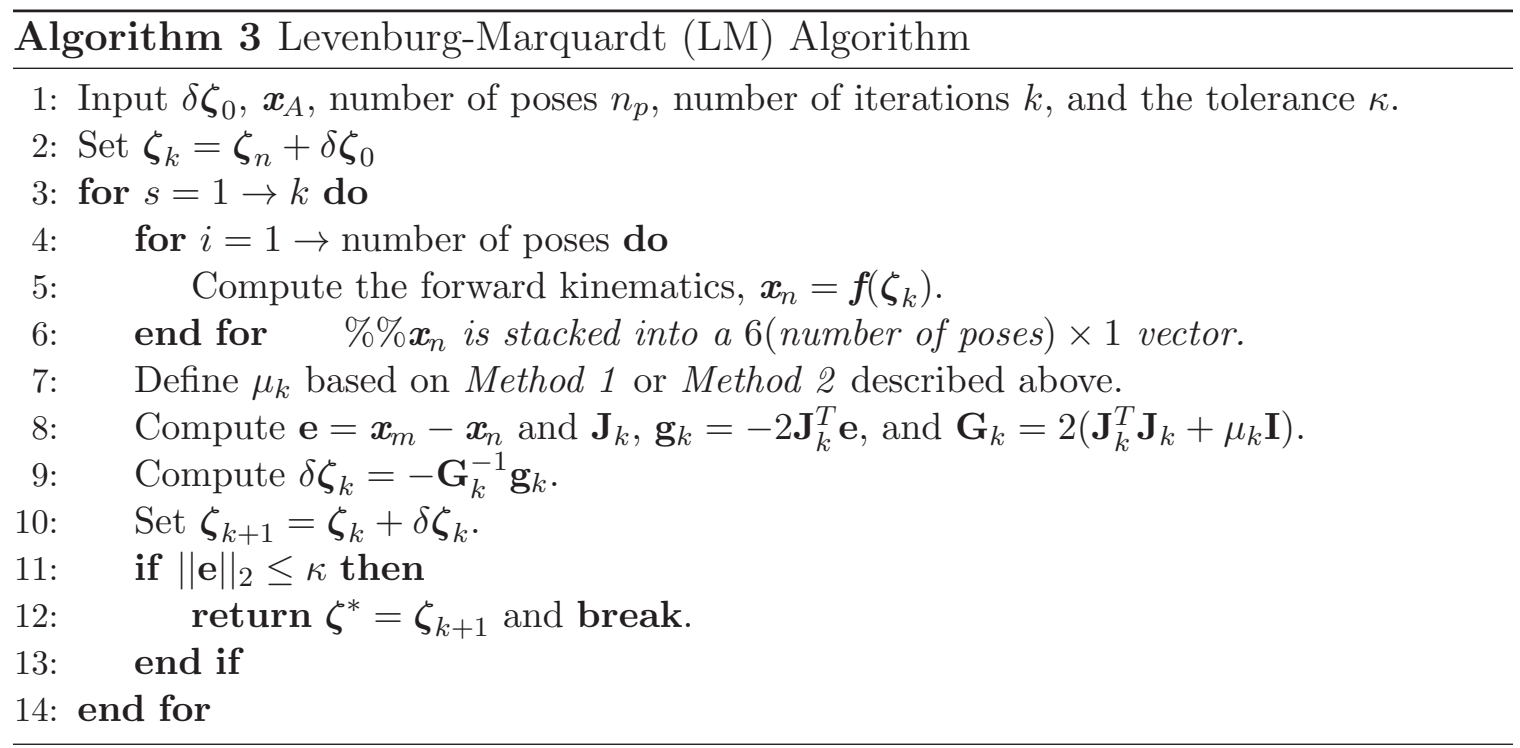

\subsubsection{Genetic Algorithm}

Inspired by the process of natural selection and genetic evolution, a genetic algorithm (GA) dynamically adapts the solution space of an optimization problem, converging towards a solution [42]. Because of the close relation to biology, and since GAs were originally applied to solve single-objective optimization problems, the objective function $L$ is referred to as fitness function. The fitness function evaluates how well each individual (possible solution) matured during GA. 
The search spaces of GA are referred to as genome and its elements are called genotypes, whereas the elements in the problem space phenome are the phenotypes. In nature, genotypes (or chromosomes) contain most or all the genetic information of the organism encoded in the chromosomal DNA. Using GAs, the genotype normally is a vector of bits, integers, or floats. A gene is a part in the genotype that contains information necessary to affect the phenotype, whereas an intron (junk DNA) is the part that does not contribute to the phenotype in any way.

GAs provide search operators that closely copy both sexual and asexual reproduction schemes from nature. In the sexual search operation (crossover reproduction), the genotypes of two parent genes will recombine into one or more offspring, mimicking the majority of natural behaviour. In the asexual search operation (mutation reproduction), mutations are the only changes made to the genotype. Mutation is performed by introducing small and random variations into the genotype, which can be achieved by randomly modifying the value of a gene. It is common to apply the properties of both search operations (sexual and asexual) for improving the fitness functions in practical applications.

A GA performs its optimization process iteratively, and each iteration is referred to as a generation. In the first generation, an initial population of random individuals (possible solutions) are created. The fitness function is evaluated for each individual in the population, and the top performing individuals are selected for reproduction (parents). In the next generation, the new individuals are created from this reproduction (children), and are evaluated for their fitness. This process repeats until a specified fitness value is met by a certain individual.

GAs are one of the most commonly used global optimization techniques with several examples involving kinematic calibration. Aoyagi [43] and $\mathrm{Wu}$ et al. [44] use GAs for selecting the best suited manipulator configuration for calibration, this 
can reduce the number of measurement points needed while maintaining satisfactory accuracy, which in turn reduces the time required on the manufacturing floor for performing a calibration.

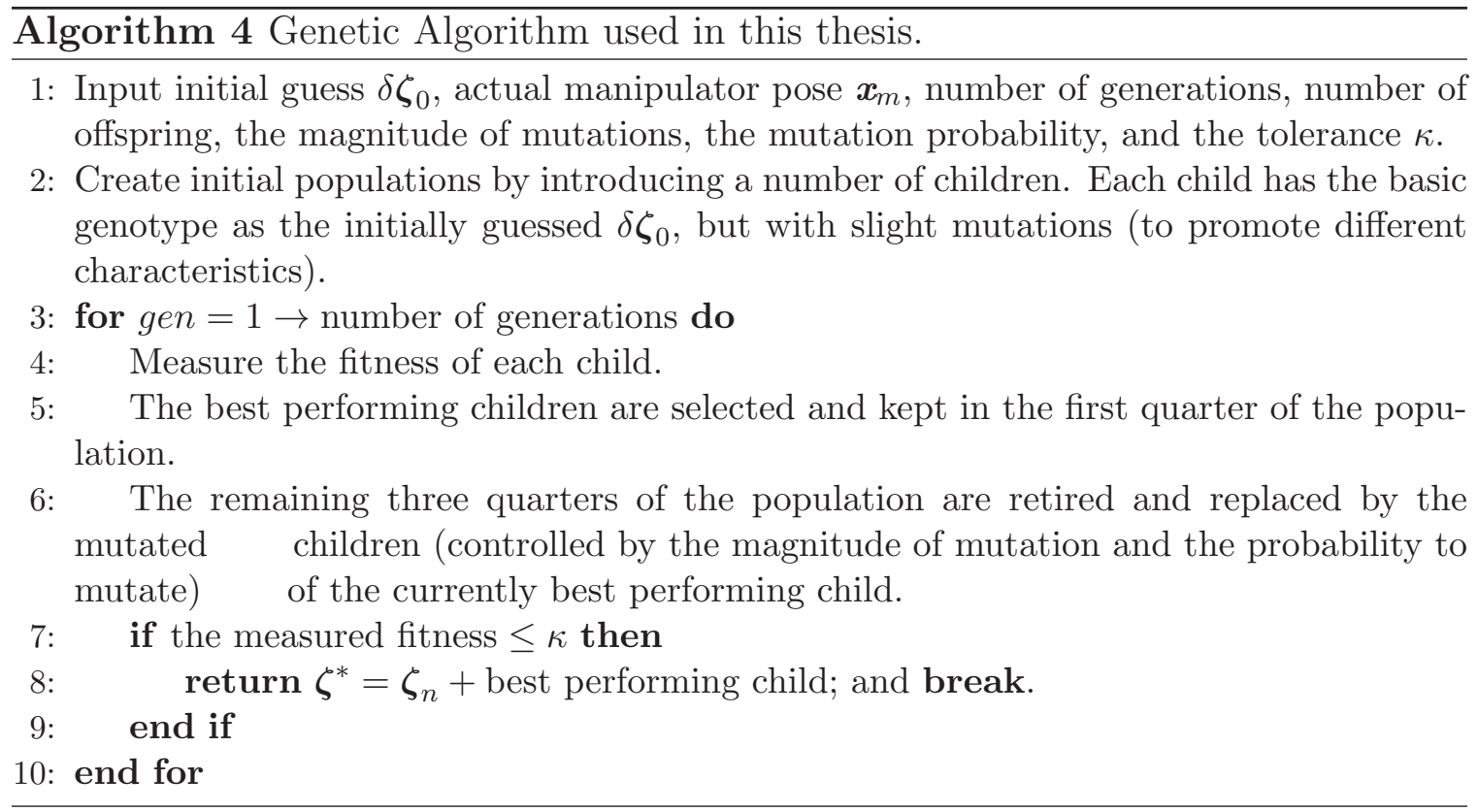

\subsubsection{Pseudoinverse using Singular Value Decomposition}

Given the approximated model as described in Eq. (2.1), the deviation $\delta \boldsymbol{x}$ can be linearized by ignoring the higher order terms, giving

$$
\delta \boldsymbol{x}=\mathbf{J} \delta \zeta
$$

The linearized system above is an overdetermined system for all kinematic calibration problems. The solution of $\delta \boldsymbol{\zeta}$ can be obtained by applying the Moore-Penrose pseudoinverse of $\mathbf{J}[45]$,

$$
\delta \zeta=\mathbf{J}^{+} \delta \boldsymbol{x}
$$


If the rows and columns of $\mathbf{J}$ are linearly independent, then $\mathbf{J}^{T} \mathbf{J}$ is invertible. In this case, Eq. (2.25) becomes

$$
\delta \boldsymbol{\zeta}=\left(\mathbf{J}^{T} \mathbf{J}\right)^{-1} \mathbf{J}^{T} \delta \boldsymbol{x} .
$$

Notice that Eq. (2.26) resembles the Gauss-Newton method in Eq. (2.22). This means that the pseudoinverse solution also exhibits the same properties as the GaussNewton method. In many practical overdetermined cases, some columns of $\mathbf{J}$ cause linear dependency, or nearly, resulting in $\mathbf{J}^{T} \mathbf{J}$ being ill-conditioned or singular.

Using singular value decomposition (SVD), every matrix can be inverted regardless of condition number [45]. SVD enables a technique for always being able to solve for $\delta \boldsymbol{\zeta}$ exactly, or estimate it with the minimum least-squares error. The matrix $\mathbf{J}$ can be decomposed into three matrix factors,

$$
\mathbf{J}=\mathbf{U} \mathbf{\Sigma} \mathbf{V}^{T}
$$

where $\mathbf{J}$ is an $M \times N$ Jacobian matrix, $\boldsymbol{\Sigma}$ is an $M \times N$ rectangular diagonal matrix with positive real singular values $\left(\sigma_{i}\right)$ on the diagonal of the uppermost $N \times N$ part of the matrix, arranged in descending order, $\mathbf{U}$ is an $M \times M$ orthogonal matrix whose orthonormal set of basis vectors corresponding to $\sigma_{i} \neq 0$ span the range of $\mathbf{J}$, and $\mathbf{V}$ is an $N \times N$ orthogonal matrix whose orthonormal columns correspond to the same numbered $\sigma_{i}=0$ are a set of basis vectors spanning the nullspace of $\mathbf{J}$. 
Because the matrices $\mathbf{U}$ and $\mathbf{V}$ are both proper orthogonal, then their inverses are equal to their transposes. The matrix $\Sigma$ is diagonal, so its inverse is simply

$$
\boldsymbol{\Sigma}^{-1}=\left[\begin{array}{cccc}
\sigma_{1}^{-1} & 0 & \cdots & 0 \\
0 & \sigma_{2}^{-1} & \cdots & 0 \\
\vdots & & \ddots & \vdots \\
0 & 0 & \cdots & \sigma_{n}^{-1} \\
0 & 0 & \cdots & 0
\end{array}\right] .
$$

Then, the Moore-Penrose pseudoinverse of $\mathbf{J}$, can be expressed as [45]

$$
\mathbf{J}^{+}=\mathbf{V} \boldsymbol{\Sigma}^{-1} \mathbf{U}^{T}
$$

The only time there are computational issues is when one or more of the $\sigma_{i}$ are either identically zero, or numerically close to zero. In this case, simply set $\sigma_{i}^{-1}=0$. This is not the desperation mathematics it appears to be, and may seem like making a bad situation worse. But, by setting $\sigma_{i}^{-1}=0$ below a threshold for $\sigma_{i}$ actually eliminates the linear combination of equations that is so corrupted by error that is, at best, useless. Because it pushes the solution vector towards infinity in the direction parallel to a nullspace vector. The last column in $\mathbf{V}$ corresponding to the eliminated $\sigma_{i}$ gives the elements of $\delta \boldsymbol{\zeta}$ that are ill-determined even if the system of equations is overdetermined. These elements of $\delta \boldsymbol{\zeta}$ are insensitive to the data [45].

The standard threshold that is normally used is to set $\sigma_{i}^{-1}=0$ if [45]

$$
\frac{\sigma_{i}}{\sigma_{\max }}<\operatorname{rank}(\mathbf{V}) \epsilon
$$


where $\epsilon$ is the machine precision, typically $\epsilon=2.2204 \times 10^{-16}$.

\subsubsection{Numerical Conditioning for Least-Squares Solutions}

Consider the linearized system represented by Eq. (2.24), and assume that $\delta \boldsymbol{x}$ contains some noise $\mathbf{b}$, which is mapped to the solution as error vector $\mathbf{a}$

$$
\delta \boldsymbol{\zeta}+\mathbf{a}=\mathbf{J}^{+}(\delta \boldsymbol{x}+\mathbf{b}) .
$$

Many mathematicians and statisticians have examined methods to numerically quantify this sensitivity with a single quantity (condition number) derived from $\mathbf{J}[46]$.

The condition number $\kappa(\mathbf{J})$ is defined to be the ratio of the largest and smallest scalar values of $\mathbf{J}$. The condition number can be used to describe the sensitivity to noise using the following ratios:

$$
\frac{\|\mathbf{a}\|_{2}}{\|\delta \boldsymbol{\zeta}\|_{2}} \leq\|\mathbf{J}\|_{2}\left\|\mathbf{J}^{-1}\right\|_{2} \frac{\|\mathbf{b}\|_{2}}{\|\delta \boldsymbol{x}\|_{2}}=\kappa(\mathbf{J}) \frac{\|\mathbf{b}\|_{2}}{\|\delta \boldsymbol{x}\|_{2}}
$$

The condition number $\kappa(\mathbf{J})$ is bounded within the range $1 \leq \kappa(\mathbf{J}) \leq \infty$, and has the following implications:

- $\kappa(\mathbf{J}) \rightarrow 1$ : if $\frac{\|\mathbf{b}\|_{2}}{\|\delta \boldsymbol{x}\|_{2}}$ is small, then $\frac{\|\mathbf{a}\|_{2}}{\|\delta \boldsymbol{\zeta}\|_{2}}$ is also small.

- $\kappa(\mathbf{J}) \rightarrow \infty$ : if $\frac{\|\mathbf{b}\|_{2}}{\|\delta \boldsymbol{x}\|_{2}}$ is small, then $\frac{\|\mathbf{a}\|_{2}}{\|\delta \boldsymbol{\zeta}\|_{2}}$ can be very large.

\subsubsection{Numerical Conditioning using SVD}

Other than the pseudoinverse, singular value decomposition can also be used as the tool for computing the condition number $\kappa(\mathbf{J})$. The diagonal matrix factor $\boldsymbol{\Sigma}$ which consists of the singular values of $\mathbf{J}$ has the form 


$$
\boldsymbol{\Sigma}=\left[\begin{array}{ccccc}
\sigma_{1} & 0 & 0 & \ldots & 0 \\
0 & \sigma_{2} & 0 & \ldots & 0 \\
\vdots & \vdots & \ddots & \ldots & \vdots \\
0 & 0 & & \ldots & \sigma_{n} \\
0 & 0 & 0 & \ldots & 0
\end{array}\right]
$$

The condition number of $\mathbf{J}$ is the ratio of the maximum and minimum singular values, and because the singular values are arranged in a descending order, then

$$
\kappa(\mathbf{J})=\frac{\sigma_{1}}{\sigma_{n}}
$$

Note that the number of $\sigma_{i} \neq 0$ in $\boldsymbol{\Sigma}$ is the $\operatorname{rank}(\mathbf{J})$.

As previously discussed, some $\sigma_{i}$ in $\Sigma$ can be numerically small, near the machine precision $\epsilon$. From Eq. (2.34), as $\sigma_{i} \rightarrow \epsilon$ then $\kappa(\mathbf{J}) \rightarrow \infty$, indicating that the estimated parameters can be poorly identified. Therefore, simply replace every $\sigma_{i} \leq \epsilon$ by 0 instead to ensure numerical stability. 


\section{Chapter 3}

\section{Kinematic Modelling}

\subsection{Properties of a Good Kinematic Model}

Everett et al. [47] proposed that a good kinematic model of a serial manipulator for calibration should exhibit three properties:

- Completeness: The minimum number of parameters required to describe all variations in the manipulator is given by

$$
C=4 R+2 P+6 .
$$

$C$ is the total number of independent parameters, $R$ is the number of revolute joints, $P$ is the number of prismatic joints, and the six is the number of parameters required to completely describe the pose of the base frame relative to the world frame.

- Proportionality: small deviations in the robot structure should be reflected by small changes in the kinematic parameters. Since most of the calibration procedures rely on numerical algorithms, a model must possess proportionality to ensure numerical stability. 
- Equivalence: the ability to transform the kinematic parameters of one model into another model. This property prevents one model from producing better results than another.

\subsection{Forward Kinematics}

The purpose of a kinematic calibration can be thought of as improving the mapping between the joint space and the task space, implying that the manipulator placement in the task space is well known. Typically, for an $n \mathrm{R}$ serial manipulator the forward kinematics modelling the manipulator maps $\Sigma_{b} \rightarrow \Sigma_{n}$ in terms of the joint angles. The $\mathrm{DH}$ convention restricts $\Sigma_{b}$ to $\Sigma_{1}$, thus additional parameters are required to place $\Sigma_{b}$ in the task space.

\subsubsection{Base Coordinate Transformation}

For the kinematic model to be complete, six additional parameters are required to specify the pose of the manipulator base frame $\Sigma_{b}$ from an arbitrarily-defined fixed world frame $\Sigma_{w}$. Figure 3.1 shows the construction of the transformation $\Sigma_{w} \rightarrow \Sigma_{b}$ (using $\left.\theta_{b}, a_{b}, d_{b}, \alpha_{b}\right)$ and the set up for $\Sigma_{1}$ (using $\theta_{1}$ and $d_{1}$ ).

These six parameters should also be included in the identification along with all the other kinematic parameters. Unlike the kinematic parameters of the manipulator, these six parameters can be poorly known and it may cause problems as the initial guess for the nonlinear optimization. Additional care is needed if the $\mathbf{z}$-axes of $\Sigma_{w}$ and $\Sigma_{b}$ are nearly parallel. 


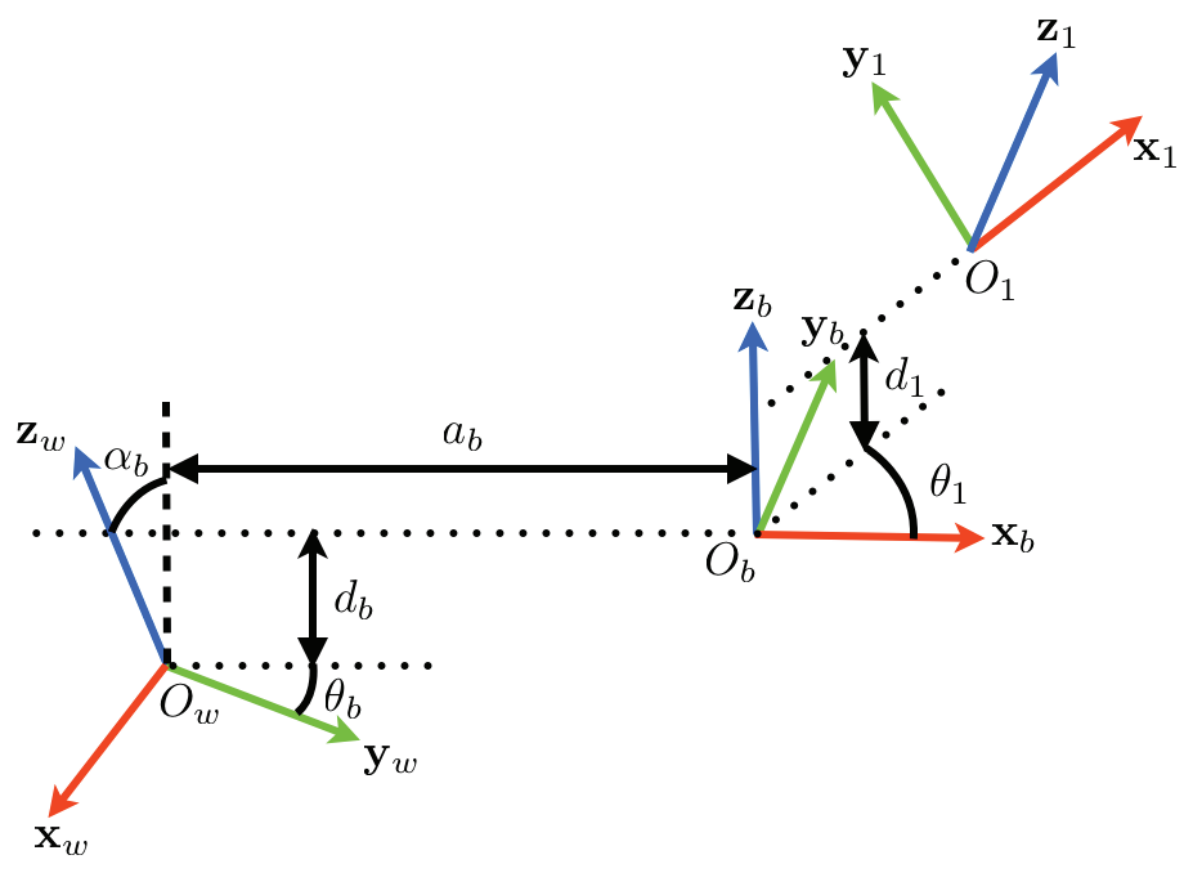

Figure 3.1: Establishing the pose of $\Sigma_{b}$ relative to $\Sigma_{w}$.

\subsubsection{End Link Coordinate Transformation}

An external metrology system typically measures the pose of some target object $\Sigma_{t}$ attached to the end-effecter. Thus for an $n$-link serial manipulator, $\Sigma_{t}=\Sigma_{n+1}$. The link placements of $\Sigma_{1} \rightarrow \Sigma_{n-1}$ are firmly established with the DH parameterization, the end-effector link frame $\Sigma_{n}$ placement is semi-arbitrary, and the end link frame $\Sigma_{n+1}$ placement is arbitrary. However, if $\mathrm{DH}$ parameters are used to describe the transformation from $\Sigma_{n-1} \rightarrow \Sigma_{n+1}$, there are a total of eight parameters. Again, only six parameters are required to describe the pose between two coordinate frames, hence two of the parameters may be set arbitrarily.

Because a calibration board is attached to the end-effector in this work, the joint axes $\mathbf{z}_{n-1}$ and $\mathbf{z}_{n+1}$ are mutually perpendicular. Then $\mathbf{z}_{n}$ and $\mathbf{z}_{n+1}$ coincide, resulting in the arbitrary choice of parameters $\alpha_{n+1}=0$ and $a_{n+1}=0$. Figure 3.2 shows the construction of the transformation $\Sigma_{n-1} \rightarrow \Sigma_{n}$ (using $\theta_{n}, a_{n}, d_{n}, \alpha_{n}$ ) and locating 


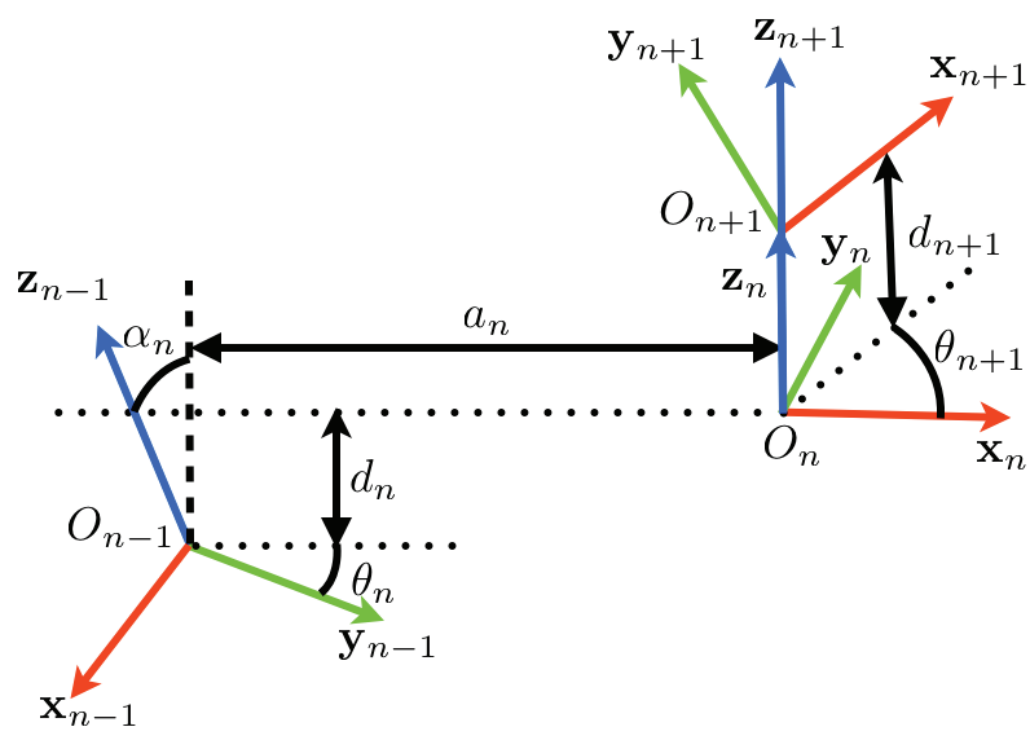

Figure 3.2: Establishing the pose of $\Sigma_{n+1}$ relative to $\Sigma_{n-1}$.

$\Sigma_{n+1}$ (using $\theta_{n+1}$ and $d_{n+1}$ ). Therefore, the parameters $\theta_{n+1}$ and $d_{n+1}$ should also be included in the kinematic calibration as well.

\subsubsection{Forward Kinematics for Serial Manipulators}

The forward kinematics of a serial manipulator is the transformation of $\Sigma_{w} \rightarrow \Sigma_{n+1}$. Using the DH Transformation or Hayati Transformation (if needed) for each link, the pose of $\Sigma_{n+1}$ can be computed by recursively multiplying the transformations of each link,

$$
{ }^{w} \mathbf{T}_{n+1}(\mathbf{q})={ }^{w} \mathbf{T}_{b}{ }^{b} \mathbf{T}_{1}\left(q_{1}\right) \ldots{ }^{n-1} \mathbf{T}_{n}\left(q_{n}\right)^{n} \mathbf{T}_{n+1},
$$

where

$$
q_{i}=\theta_{i}+\theta_{o, i}
$$

$\theta_{i}$ is the joint angle read from each joint encoder, and $\theta_{o, i}$ is the joint offset due to the misalignment of each joint from its zero position. 
Eq. (3.2) can be written in the compact form,

$$
{ }^{w} \boldsymbol{x}_{n+1, k}=\left[\begin{array}{c}
{ }^{w} \mathbf{p}_{n+1, k} \\
{ }^{w} \boldsymbol{\Phi}_{n+1, k}
\end{array}\right]=\boldsymbol{f}\left(\boldsymbol{\zeta}_{k}\right)
$$

where $\boldsymbol{\zeta}_{k}=\left[\boldsymbol{\theta}_{o, k}, \boldsymbol{a}_{k}, \boldsymbol{d}_{k}, \boldsymbol{\alpha}_{k}\right]^{T}$, and they are treated as variables, while $\boldsymbol{\theta}$ is treated as constant. The position component ${ }^{w} \mathbf{p}_{n+1}$ is extracted from the first three components of the last column in ${ }^{w} \mathbf{T}_{n+1}$. The orientation component ${ }^{w} \boldsymbol{\Phi}_{n+1}$ is extracted from the first $3 \times 3$ portion of ${ }^{w} \mathbf{T}_{n+1}$.

\subsection{Jacobian Matrix for Kinematic Calibration}

The derivation of the Jacobian matrix is covered in most introductory robotics texts, including $[12,32,48,49]$. The Jacobian matrix can be obtained either analytically or geometrically.

\subsubsection{Analytical Jacobian Matrix}

Veitschegger and $\mathrm{Wu}[28]$ assumed that all variations about the nominal kinematic parameters are accounted for in $\delta \boldsymbol{\zeta}_{i}=\left[\delta \theta_{o, i}, \delta a_{i}, \delta d_{i}, \delta \alpha_{i}\right]^{T}$. By linearizing Eq. (2.8)

$$
\delta^{i-1} \mathbf{T}_{i}=\frac{\partial^{i-1} \mathbf{T}_{i}}{\partial \theta_{o, i}} \delta \theta_{o, i}+\frac{\partial^{i-1} \mathbf{T}_{i}}{\partial a_{i}} \delta a_{i}+\frac{\partial^{i-1} \mathbf{T}_{i}}{\partial d_{i}} \delta d_{i}+\frac{\partial^{i-1} \mathbf{T}_{i}}{\partial \alpha_{i}} \delta \alpha_{i}
$$

Because the matrix being differentiated is in the form of a homogeneous transformation matrix,

$$
\frac{\partial^{i-1} \mathbf{T}_{i}}{\partial \rho_{i}}={ }^{i-1} \Delta_{\rho_{i}, i-1}{ }^{i-1} \mathbf{T}_{i}={ }^{i-1} \mathbf{T}_{i}{ }^{i} \Delta_{\rho_{i}, i}
$$

where $\rho$ is just a symbol that can be replaced by $\theta_{o}, a, d$, or $\alpha$. 
The coefficient matrix, ${ }^{i-1} \Delta_{i-1}$ or ${ }^{i} \Delta_{i}$, is termed the velocity coefficient matrix [49]. The velocity coefficient matrices for each kinematic parameter are as follow:

$$
\begin{aligned}
& { }^{i-1} \Delta_{\theta_{i}, i-1}=\left[\begin{array}{cccc}
0 & -1 & 0 & 0 \\
1 & 0 & 0 & 0 \\
0 & 0 & 0 & 0 \\
0 & 0 & 0 & 0
\end{array}\right]=\left[\begin{array}{cc}
{ }^{i-1} \tilde{\boldsymbol{k}}_{i-1} & 0 \\
0 & 0
\end{array}\right], \\
& { }^{i-1} \Delta_{d_{i}, i-1}=\left[\begin{array}{cccc}
0 & 0 & 0 & 0 \\
0 & 0 & 0 & 0 \\
0 & 0 & 0 & 1 \\
0 & 0 & 0 & 0
\end{array}\right]=\left[\begin{array}{cc}
\mathbf{0}^{i-1} \hat{\boldsymbol{k}}_{i-1} \\
\mathbf{0} & 0
\end{array}\right] \text {, } \\
& { }^{i-1} \Delta_{a_{i}, i-1}=\left[\begin{array}{cccc}
0 & 0 & 0 & \cos \theta_{i} \\
0 & 0 & 0 & \sin \theta_{i} \\
0 & 0 & 0 & 0 \\
0 & 0 & 0 & 0
\end{array}\right] \\
& { }^{i-1} \Delta_{\alpha_{i}, i-1}=\left[\begin{array}{cccc}
0 & 0 & \sin \theta_{i} & -d_{i} \sin \theta_{i} \\
0 & 0 & -\cos \theta_{i} & d_{i} \cos \theta_{i} \\
-\sin \theta_{i} & \cos \theta_{i} & 0 & 0 \\
0 & 0 & 0 & 0
\end{array}\right] .
\end{aligned}
$$


Because the forward kinematics of the manipulator given by Eq. (3.2) is the transformation from $\Sigma_{w} \rightarrow \Sigma_{n+1}$, the infinitesimal transformation matrix given by Eq. (3.6) should also reflect this property as

$$
\frac{\partial^{w} \mathbf{T}_{n+1}}{\partial \boldsymbol{\rho}}=\sum_{i=1}^{n+1}{ }^{w} \mathbf{T}_{b}{ }^{b} \mathbf{T}_{1} \ldots \frac{\partial^{i-1} \mathbf{T}_{i}}{\partial \rho_{i}} \cdots^{n-1} \mathbf{T}_{n}{ }^{n} \mathbf{T}_{n+1}
$$

which also has the form of a $4 \times 4$ matrix,

$$
\frac{\partial^{w} \mathbf{T}_{n+1}}{\partial \boldsymbol{\rho}}=\left[\begin{array}{cccc}
\frac{\partial^{w} n_{x, n+1}}{\partial \boldsymbol{\rho}} & \frac{\partial^{w} s_{x, n+1}}{\partial \boldsymbol{\rho}} & \frac{\partial^{w} a_{x, n+1}}{\partial \boldsymbol{\rho}} & \frac{\partial^{w} p_{x, n+1}}{\partial \boldsymbol{\rho}} \\
\frac{\partial^{w} n_{y, n+1}}{\partial \boldsymbol{\rho}} & \frac{\partial^{w} s_{y, n+1}}{\partial \boldsymbol{\rho}} & \frac{\partial^{w} a_{y, n+1}}{\partial \boldsymbol{\rho}} & \frac{\partial^{w} p_{y, n+1}}{\partial \boldsymbol{\rho}} \\
\frac{\partial^{w} n_{z, n+1}}{\partial \boldsymbol{\rho}} & \frac{\partial^{w} s_{z, n+1}}{\partial \boldsymbol{\rho}} & \frac{\partial^{w} a_{z, n+1}}{\partial \boldsymbol{\rho}} & \frac{\partial^{w} p_{z, n+1}}{\partial \boldsymbol{\rho}} \\
0 & 0 & 0 & 0
\end{array}\right] .
$$

Similar to the compact form of the forward kinematics, Eq. (3.4), the infinitesimal transformation matrix (linearized model), Eq. (3.8), should also be expressed in compact form with the same corresponding elements,

$$
\mathbf{J}_{A}=\left[\begin{array}{c}
\frac{\partial^{w} \mathbf{p}_{n+1, k}}{\partial \boldsymbol{\rho}} \\
\frac{\partial^{w} \boldsymbol{\Phi}_{n+1, k}}{\partial \boldsymbol{\rho}}
\end{array}\right]=\frac{\partial \boldsymbol{f}\left(\boldsymbol{\zeta}_{k}\right)}{\partial \boldsymbol{\rho}} .
$$

\subsubsection{Geometric Jacobian Matrix}

For kinematic calibration, it is important to compute the Jacobian matrix quickly. Directly computing Eq. (3.6) for each kinematic parameter can be quite time consuming. Fortunately for manipulators, the Jacobian matrix can be directly related to the velocity of each manipulator link. 
Each variation in the DH parameters is a degree of freedom (DOF) about a specific axis. The angular and linear joint displacements in the $\mathbf{z}_{i-1}$-axis, is represented by $\theta_{i}$ and $d_{i}$, respectively. Whereas $\alpha_{i}$ and $a_{i}$ represent the angular and linear joint displacements in the $\mathbf{x}_{i}$-axis, respectively. This means that the geometric Jacobian must solve the linear and angular velocities of $\Sigma_{n+1}$ in terms of the joint rates.

The identification Jacobian matrix can be partitioned into

$$
\mathbf{J}_{G}=\left[\begin{array}{l}
\mathcal{J}_{p} \\
\mathcal{J}_{o}
\end{array}\right]=\left[\begin{array}{cccc}
\mathcal{J}_{p, \boldsymbol{\theta}} & \mathcal{J}_{p, \boldsymbol{a}} & \mathcal{J}_{p, \boldsymbol{d}} & \mathcal{J}_{p, \boldsymbol{\alpha}} \\
\mathcal{J}_{o, \boldsymbol{\theta}} & \mathcal{J}_{o, \boldsymbol{a}} & \mathcal{J}_{o, \boldsymbol{d}} & \mathcal{J}_{o, \boldsymbol{\alpha}}
\end{array}\right]
$$

where

$$
\begin{aligned}
& {\left[\begin{array}{c}
\mathcal{J}_{p, \theta_{i}} \\
\mathcal{J}_{o, \theta_{i}}
\end{array}\right]=\left[\begin{array}{c}
{ }^{w} \hat{\boldsymbol{k}}_{i-1} \times{ }^{i-1} \boldsymbol{p}_{n+1} \\
{ }^{w} \hat{\boldsymbol{k}}_{i-1}
\end{array}\right],} \\
& {\left[\begin{array}{c}
\mathcal{J}_{p, a_{i}} \\
\mathcal{J}_{o, a_{i}}
\end{array}\right]=\left[\begin{array}{c}
{ }^{w} \hat{\boldsymbol{i}}_{i} \\
\mathbf{0}
\end{array}\right]} \\
& {\left[\begin{array}{c}
\mathcal{J}_{p, d_{i}} \\
\mathcal{J}_{o, d}
\end{array}\right]=\left[\begin{array}{c}
{ }^{w} \hat{\boldsymbol{k}}_{i-1} \\
\mathbf{0}
\end{array}\right],} \\
& {\left[\begin{array}{c}
\mathcal{J}_{p, \alpha_{i}} \\
\mathcal{J}_{o, \alpha_{i}}
\end{array}\right]=\left[\begin{array}{c}
{ }^{w} \hat{\boldsymbol{i}}_{i} \times{ }^{i} \boldsymbol{p}_{n+1} \\
{ }^{w} \hat{\boldsymbol{i}}_{i}
\end{array}\right],}
\end{aligned}
$$

${ }^{w} \hat{\boldsymbol{k}}_{i-1}$ and ${ }^{w} \hat{\boldsymbol{i}}_{i}$ are the unit vectors representing the $\mathbf{z}$-axis of ${ }^{w} \mathbf{R}_{i-1}$ and the $\mathbf{X}$-axis of ${ }^{w} \mathbf{R}_{i}$, respectively. 


\subsubsection{Deriving the Geometric Jacobian from the Analytical Jacobian}

Recall that the partial derivative of a homogeneous transformation matrix is the multiplication of the velocity coefficient matrix and the homogeneous transformation matrix. Then each component of the infinitesimal transformation matrix can now be expressed in the form of

$$
{ }^{w} \mathbf{T}_{b}{ }^{b} \mathbf{T}_{1} \ldots \frac{\partial^{i-1} \mathbf{T}_{i}}{\partial \rho_{i}} \ldots{ }^{n-1} \mathbf{T}_{n}{ }^{n} \mathbf{T}_{n+1}=\mathcal{C}_{\rho, i}{ }^{w} \mathbf{T}_{n+1}
$$

where

$$
\begin{aligned}
\mathcal{C}_{\rho, i} & =\left[{ }^{w} \mathbf{T}_{b}{ }^{b} \mathbf{T}_{1}{ }^{1} \mathbf{T}_{2} \ldots{ }^{i-2} \mathbf{T}_{i-1}\right] \frac{\partial^{i-1} \mathbf{T}_{i}}{\partial \rho_{i}}\left[{ }^{w} \mathbf{T}_{b}{ }^{b} \mathbf{T}_{1}{ }^{1} \mathbf{T}_{2} \ldots{ }^{i-1} \mathbf{T}_{i}\right]^{-1} \\
& =\left[{ }^{w} \mathbf{T}_{b}{ }^{b} \mathbf{T}_{1}{ }^{1} \mathbf{T}_{2} \ldots{ }^{i-2} \mathbf{T}_{i-1}\right]^{i-1} \Delta_{\rho, i-1}{ }^{i-1} \mathbf{T}_{i}\left[{ }^{w} \mathbf{T}_{b}{ }^{b} \mathbf{T}_{1}{ }^{1} \mathbf{T}_{2} \ldots{ }^{i-1} \mathbf{T}_{i}\right]^{-1}, \\
& =\left[{ }^{w} \mathbf{T}_{b}{ }^{b} \mathbf{T}_{1}{ }^{1} \mathbf{T}_{2} \ldots{ }^{i-2} \mathbf{T}_{i-1}\right]^{i-1} \Delta_{\rho, i-1}\left[{ }^{w} \mathbf{T}_{b}{ }^{b} \mathbf{T}_{1}{ }^{1} \mathbf{T}_{2} \ldots{ }^{i-2} \mathbf{T}_{i-1}\right]^{-1}
\end{aligned}
$$

Once each $\mathcal{C}_{\rho, i}$ is expanded and simplified into a $4 \times 4$ matrix, there are six independent components in each matrix which can be rearranged into a $6 \times 1$ vector like the compact form of the forward kinematics in Eq. (3.4). This vector $\boldsymbol{c}_{\rho, i}$ has the form of

$$
\boldsymbol{c}_{\rho, i}=\left\{\begin{array}{cc}
{\left[\begin{array}{cc}
{ }^{w} \hat{\boldsymbol{k}}_{i-1} \times\left({ }^{w} \boldsymbol{p}_{n+1}-{ }^{w} \boldsymbol{p}_{i-1}\right) \\
{ }^{w} \hat{\boldsymbol{k}}_{i-1}
\end{array}\right]} & \text { for } \theta, \\
{\left[\begin{array}{c}
{ }^{w} \hat{\boldsymbol{k}}_{i-1} \\
\mathbf{0}
\end{array}\right]} & \text { for } d .
\end{array}\right.
$$

For the case of the other two DH parameters, $a$ and $\alpha$, the derivation of $\mathcal{C}_{\rho, i}$ has to be treated differently. The velocity coefficient matrices ${ }^{i-1} \Delta_{i-1}$ for $a$ and $\alpha$ are not 
constant, recall Eqs. (3.7c) and (3.7d). However, because of the way that $a$ and $\alpha$ are defined, the velocity coefficient matrix is only constant when

$$
\begin{aligned}
{ }^{i} \Delta_{a_{i}, i} & =\left[\begin{array}{cccc}
0 & 0 & 0 & 1 \\
0 & 0 & 0 & 0 \\
0 & 0 & 0 & 0 \\
0 & 0 & 0 & 0
\end{array}\right]=\left[\begin{array}{ll}
\mathbf{0} & { }^{i} \hat{\boldsymbol{i}}_{i} \\
\mathbf{0} & 0
\end{array}\right], \\
{ }^{i} \Delta_{\alpha_{i}, i}= & {\left[\begin{array}{llll}
0 & 0 & 0 & 0 \\
0 & 0 & -1 & 0 \\
0 & 1 & 0 & 0 \\
0 & 0 & 0 & 0
\end{array}\right]=\left[\begin{array}{ll}
{ }^{i} \boldsymbol{i}_{i} & 0 \\
\mathbf{0} & 0
\end{array}\right] . }
\end{aligned}
$$

Then the coefficient $\mathcal{C}_{\rho, i}$ must be rearranged to reflect this change:

$$
\begin{aligned}
& \mathcal{C}_{\rho, i}=\left[{ }^{w} \mathbf{T}_{b}{ }^{b} \mathbf{T}_{1}{ }^{1} \mathbf{T}_{2} \ldots{ }^{i-2} \mathbf{T}_{i-1}\right] \frac{\partial^{i-1} \mathbf{T}_{i}}{\partial \rho_{i}}\left[{ }^{w} \mathbf{T}_{b}{ }^{b} \mathbf{T}_{1}{ }^{1} \mathbf{T}_{2} \ldots{ }^{i-1} \mathbf{T}_{i}\right]^{-1} \\
& =\left[{ }^{w} \mathbf{T}_{b}{ }^{b} \mathbf{T}_{1}{ }^{1} \mathbf{T}_{2} \ldots{ }^{i-2} \mathbf{T}_{i-1}\right]^{i-1} \mathbf{T}_{i}{ }^{i} \Delta_{\rho, i}\left[{ }^{w} \mathbf{T}_{b}{ }^{b} \mathbf{T}_{1}{ }^{1} \mathbf{T}_{2} \ldots{ }^{i-1} \mathbf{T}_{i}\right]^{-1} \\
& =\left[{ }^{w} \mathbf{T}_{b}{ }^{b} \mathbf{T}_{1}{ }^{1} \mathbf{T}_{2} \ldots{ }^{i-1} \mathbf{T}_{i}\right]^{i} \Delta_{\rho, i}\left[{ }^{w} \mathbf{T}_{b}^{b} \mathbf{T}_{1}{ }^{1} \mathbf{T}_{2} \ldots{ }^{i-1} \mathbf{T}_{i}\right]^{-1}, \\
& \boldsymbol{c}_{i}=\left\{\begin{array}{cc}
{\left[\begin{array}{c}
{ }^{w} \hat{\boldsymbol{i}}_{i} \\
0
\end{array}\right]} & \text { for } a, \\
{\left[\begin{array}{c}
{ }^{w} \hat{\boldsymbol{i}}_{i} \times\left({ }^{w} \boldsymbol{p}_{n+1}-{ }^{w} \boldsymbol{p}_{i}\right) \\
{ }^{w} \hat{\boldsymbol{i}}_{i}
\end{array}\right]} & \text { for } \alpha .
\end{array}\right.
\end{aligned}
$$




\subsubsection{Jacobian for the Hayati Parameters}

The Hayati parameters include parameter $\beta_{i}$ accounting for a DOF about the $\mathbf{y}$-axis of $\Sigma_{i}$. The Hayati Transformation from Eq. (2.9) can be linearized as

$$
\delta^{i-1} \mathbf{T}_{i}=\frac{\partial^{i-1} \mathbf{T}_{i}}{\partial \theta_{o, i}} \delta \theta_{o, i}+\frac{\partial^{i-1} \mathbf{T}_{i}}{\partial a_{i}} \delta a_{i}+\frac{\partial^{i-1} \mathbf{T}_{i}}{\partial \alpha_{i}} \delta \alpha_{i}+\frac{\partial^{i-1} \mathbf{T}_{i}}{\partial \beta_{i}} \delta \beta_{i}
$$

All the parameters, except for $\beta_{i}$, are identical to the DH parameters, thus the partial derivative of Eq. (2.9) with respect to $\beta_{i}$ is

$$
\frac{\partial^{i-1} \mathbf{T}_{i}}{\partial \beta_{i}}={ }^{i-1} \mathbf{T}_{i}^{i} \Delta_{\beta_{i}, i}
$$

where

$$
{ }^{i} \Delta_{\beta, i}=\left[\begin{array}{cccc}
0 & 0 & 1 & 0 \\
0 & 0 & 0 & 0 \\
-1 & 0 & 0 & 0 \\
0 & 0 & 0 & 0
\end{array}\right]=\left[\begin{array}{cc}
i^{\tilde{j}_{i}} & 0 \\
\mathbf{0} & 1
\end{array}\right] .
$$

Because the Hayati parameters can only be used for the link with nominally parallel joint axes. The infinitesimal transformation matrix from Eq. (3.8) must account for Eq. (3.19) correctly. However, it is much simpler to geometrically determine the Jacobian matrix:

$$
\left[\begin{array}{c}
\mathcal{J}_{p, \beta_{i}} \\
\mathcal{J}_{o, i}
\end{array}\right]=\left[\begin{array}{c}
{ }^{w} \hat{\boldsymbol{j}}_{i} \times{ }^{i} \boldsymbol{p}_{n+1} \\
{ }^{w} \hat{\boldsymbol{j}}_{i}
\end{array}\right] .
$$




\subsection{Differential Kinematics for the Objective Function}

The objective function $L$ is the Eculidean 2-norm of the errors e observed between the actual pose $\mathbf{x}_{A}$ and the predicted pose $\mathbf{x}_{B}$. For the kinematic calibration problem, $L$ is minimized at the best solution, $\delta \boldsymbol{\zeta}^{*}$. The error e must be formulated according to the method used (absolute or relative measurement), i.e. Eq. (2.2) or (2.6).

Considering Eq. (2.1), as $\mathbf{e} \rightarrow 0$ then $\delta \boldsymbol{x} \rightarrow \delta \boldsymbol{x}^{*}$, implying that $\mathbf{e}$ is directly related to $\mathbf{J}$. The errors e are composed of position and orientation components. The position errors, $\mathbf{e}_{p}$, are contained in the last column of Eq. (3.9),

$$
\mathbf{e}_{p}=\mathbf{p}_{A}-\mathbf{p}_{B}
$$

The orientation error, $\mathbf{e}_{o}$, is directly related to the orientation component, the upper left $3 \times 3$ portion of the matrix represented by Eq. (3.9). The three columns of this proper orthogonal rotation matrix are the partial derivatives of unit vectors. This yields nine elements, of which only three are independent. The six dependent elements can lead to configurations that cause representational singularities in $\mathbf{J}$, which may cause divergence in the error identification.

It is common to convert the rotational matrix from the homogeneous transformation matrix of the forward kinematics to the ZYX Euler angles $(\phi, \theta, \psi)$. However, there also exist the representational singularities that can occur when $\theta=\frac{\pi}{2}$, since $\tan \left(\frac{n \pi}{2}\right)$ approaches an asymptote where $n=1,2, \ldots, N$. Therefore, some errors in the orientation may not be observed using this representation. 
Fortunately for kinematic calibration problems, there is another method to represent orientation errors in vector quantities with minimal representation. Instead of relying on determining the differences of finite angles, consider the orientation errors as infinitesimal rotations. Using the orientation of the end-effector represented using ZYX rotation as shown in the upper $3 \times 3$ portion of Eq. (A.23), assuming that all the associated angles causing the rotation are infinitesimal (implying that $\theta_{i} \approx \delta \theta_{i}$, where $i=x, y, z)$, then

$$
\mathbf{R}_{Z Y X} \approx\left[\begin{array}{ccc}
1 & -\delta \theta_{z} & \delta \theta_{y} \\
\delta \theta_{z} & 1 & -\delta \theta_{x} \\
-\delta \theta_{y} & \delta \theta_{x} & 1
\end{array}\right]
$$

Suppose the models A and B have the orientations represented by the frame $\Sigma_{A}$ and $\Sigma_{B}$, respectively. The relationship between the two frames is

$$
\Sigma_{B}=\mathbf{R}_{Z Y X} \Sigma_{A}
$$

By premultiplying both sides by $\mathbf{R}_{Z Y X}^{T}$, then

$$
\begin{aligned}
\Sigma_{A} & =\Sigma_{B}+\Delta \Sigma=\mathbf{R}_{Z Y X}^{T} \Sigma_{B}, \\
& \approx\left[\begin{array}{ccc}
1 & \delta \theta_{z} & -\delta \theta_{y} \\
-\delta \theta_{z} & 1 & \delta \theta_{x} \\
\delta \theta_{y} & -\delta \theta_{x} & 1
\end{array}\right] \Sigma_{B} \approx\left[\begin{array}{ccc}
1 & 0 & 0 \\
0 & 1 & 0 \\
0 & 0 & 1
\end{array}\right] \Sigma_{B}-\left[\begin{array}{rrr}
0 & -\delta \theta_{z} & \delta \theta_{y} \\
\delta \theta_{z} & 0 & -\delta \theta_{x} \\
-\delta \theta_{y} & \delta \theta_{x} & 0
\end{array}\right] \Sigma_{B},
\end{aligned}
$$


which leads to

$$
\Delta \Sigma \approx \Delta \tilde{\boldsymbol{\theta}} \Sigma_{B}
$$

where

$$
\Delta \tilde{\boldsymbol{\theta}}=\left[\begin{array}{ccc}
0 & -\delta \theta_{z} & \delta \theta_{y} \\
\delta \theta_{z} & 0 & -\delta \theta_{x} \\
-\delta \theta_{y} & \delta \theta_{x} & 0
\end{array}\right],
$$

is a skew symmetric matrix. This skew symmetric matrix resembles the angular velocities in Eq. (B.6) (refer to Appendix B). Hence, Eq. (3.28) can be thought of as the infinitesimal displacement version of the angular velocity vector $\boldsymbol{\omega}$.

Numerical estimation of $\boldsymbol{\Delta} \tilde{\boldsymbol{\theta}}$ can cause the diagonal elements to be non-zero. The off-diagonal components that are supposedly equal in magnitude may exhibit some differences. For the work in this thesis, a function written in MATLABR ignores the diagonal elements and averages the skew symmetric elements, yielding the orientation error,

$$
\mathbf{e}_{o}=\left[\begin{array}{lll}
\delta \theta_{x} & \delta \theta_{y} & \delta \theta_{z}
\end{array}\right]^{T}
$$




\section{Chapter 4}

\section{Simulations For Kinematic Calibration}

\subsection{The Kinematic Models}

Two manipulators were used in this work, a Thermo CRS A465 and a 7 DOF WAM Arm. The kinematic models of both manipulators were set up using the DH convention or the Hayati convention where necessary.

Table 4.1 summarizes the complete model of the A465 based on the frames assigned in Figure 4.1. The nominal values of the kinematic parameters are provided in the user manual [50]. For the A465, joint axes 2 and 3 are designed to be parallel and hence the Hayati convention is used to define link 3.

Table 4.1: The DH parameters for the Thermo CRS A465.

\begin{tabular}{cccccc}
\hline Link Number & $\theta_{o, i}[\mathrm{deg}]$. & $a_{i}[\mathrm{~mm}]$ & $d_{i}[\mathrm{~mm}]$ & $\alpha_{i}[\mathrm{deg}]$. & $\beta_{i}[\mathrm{deg}]$. \\
\hline \hline Base & 0 & 0 & 0 & 0 & - \\
1 & 0 & 0 & 330 & 90 & - \\
2 & 90 & 303 & 0 & 0 & - \\
3 & 90 & 0 & 0 & 90 & 0 \\
4 & 0 & 0 & 330 & -90 & - \\
5 & 0 & 0 & 0 & 90 & - \\
6 & 0 & 0 & 76 & 0 & - \\
End Link & 0 & 0 & 0 & 0 & - \\
\hline
\end{tabular}




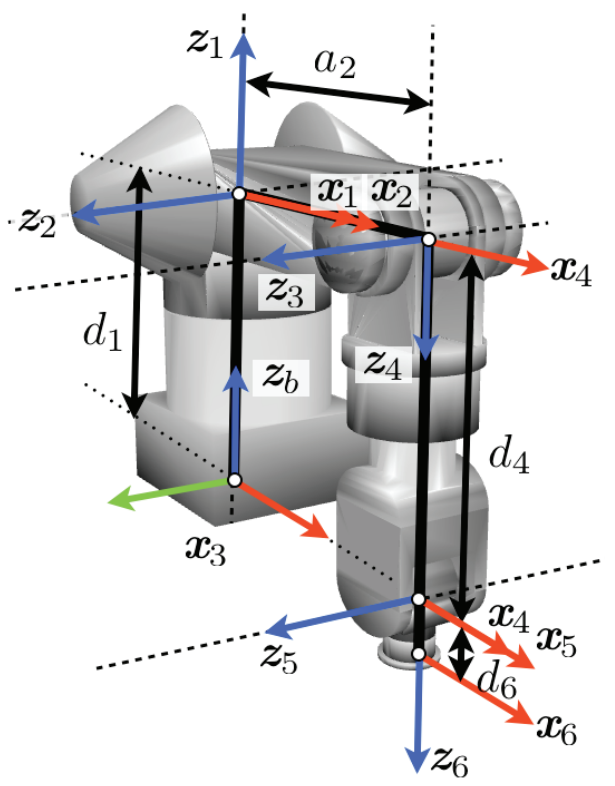

Figure 4.1: The DH parameter assignment for the CRS A465 shown in its zero position.

Table 4.2 summarizes the kinematic model of the 7 DOF WAM Arm using Figure 4.2 as a reference for the assigned coordinate frames. The nominal values of the kinematic parameters are found in the user manual [51]. The WAM does not need Hayati parameters for any links, because all neighbouring joint axes are nominally orthogonal.

Table 4.2: The DH parameters for the 7 DOF WAM Arm.

\begin{tabular}{ccccc}
\hline Link Number & $\theta_{o, i}[\mathrm{deg}]$. & $a_{i}[\mathrm{~mm}]$ & $d_{i}[\mathrm{~mm}]$ & $\alpha_{i}[\mathrm{deg}]$. \\
\hline \hline Base & 0 & 0 & 0 & 0 \\
1 & 0 & 0 & 346 & -90 \\
2 & 0 & 0 & 0 & 90 \\
3 & 0 & 45 & 550 & -90 \\
4 & 0 & -45 & 0 & 90 \\
5 & 0 & 0 & 300 & -90 \\
6 & 0 & 0 & 0 & 90 \\
7 & 0 & 0 & 60 & 0 \\
End Link & 0 & 0 & 0 & 0 \\
\hline
\end{tabular}




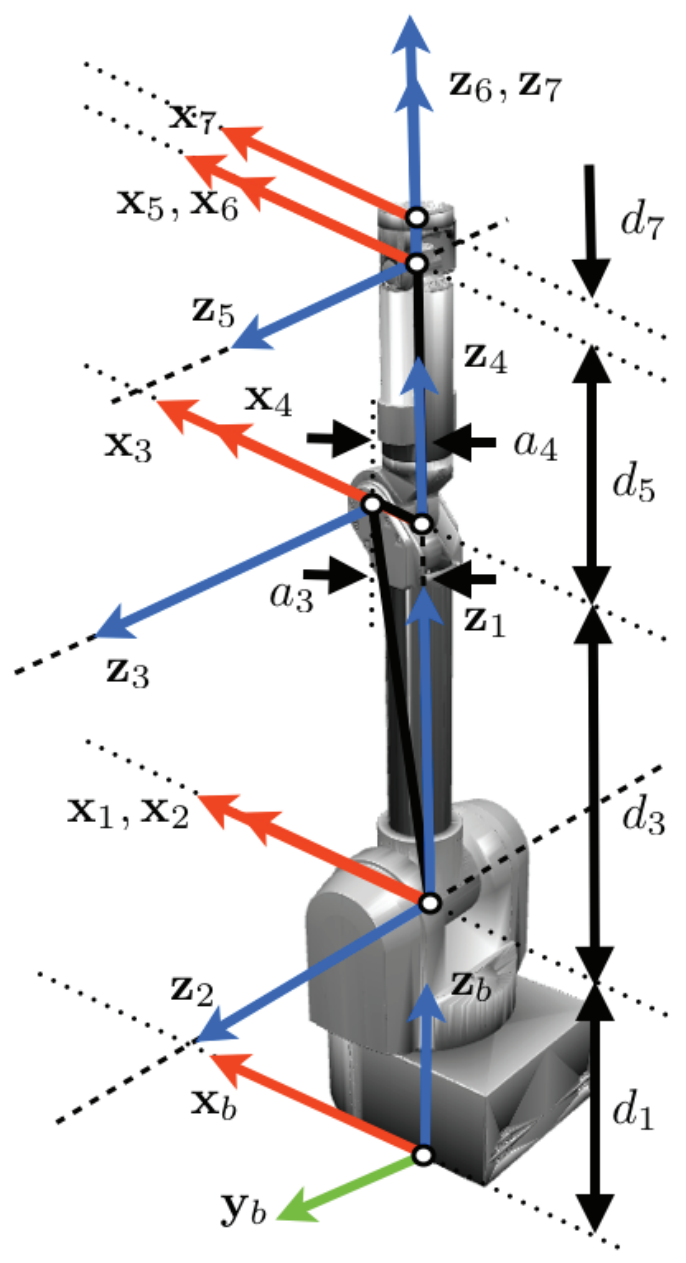

Figure 4.2: The DH parameters assignment of the WAM Arm shown in its zero position.

\subsection{Simulation Example}

A MATLABR, program was written to perform kinematic calibration simulations to compare the absolute and relative measurement concepts. The main purpose of this simulation is to develop and test the algorithms before performing a time consuming experiment. Only the kinematic model of the A465 was used in the simulation, because it possesses parallel consecutive joint axes, which tend to be problematic for kinematic calibration using numerical algorithms. 


\subsubsection{Measurements}

The actual pose $\boldsymbol{x}_{A}$ can be modelled using Eq. (3.4) and the kinematic parameters $\boldsymbol{\zeta}_{A}$. The $\boldsymbol{\zeta}_{A}$ of the A465 are simulated by assuming a Gaussian distribution of noise about the nominal kinematic parameters $\boldsymbol{\zeta}_{n}$,

$$
\zeta_{A}=\mathcal{N}\left(\zeta_{n}, \bar{\sigma}_{\zeta}\right)
$$

where $\bar{\sigma}_{\zeta}$ is the standard deviation of the Gaussian distribution. The numerical values used in $\bar{\sigma}_{\zeta}$ were $5 \mathrm{~mm}$ for length parameters and $5^{\circ}$ for angular parameters.

In order to perform kinematic calibration, a set of measurement configurations were simulated by randomly generating joint angles. The joint angles $\boldsymbol{\theta}_{i}$ configuration for each pose were randomly selected within the limits of each joint (provided in the user manual [50]). Then $\boldsymbol{x}_{A i}$ can be computed by substituting $\boldsymbol{\theta}_{i}$ and $\boldsymbol{\zeta}_{A}$ into Eq. (3.4). The number of measured poses was set to be 50 in the simulation, which means that $i \in\{1,2, \cdots, 50\}$.

\subsubsection{Absolute Measurement Results}

The simulation presented here is the kinematic calibration using the absolute measurement concept (AMC). The solution of this simulation is identified using the SVD approximation, and the results are summarized in Table 4.3 and Table 4.4 for the DH and the Hayati conventions, respectively. Both conventions took 17 iterations to converge to errors below the tolerance set for the objective function, and $\operatorname{rank}(\mathbf{J})$ for both are 28. The former, as expected, identified 28 out of 28 parameters correctly, and the latter yielded 27 out of 28 correctly identified parameters. This means that the smallest singular value $\sigma_{n}$ in $\mathbf{J}$ is indeed small but still greater than the $\operatorname{rank}(V) \epsilon$. 
As can be seen in the Table $4.4, \delta \beta_{3}$ was not identified correctly and it exhibited $90 \%$ percent error. This is because $\delta \beta_{3}$ is poorly defined and is actually a redundant parameter for defining two arbitrary frames. As previously mentioned for the case of parallel consecutive joint axes, the $d_{i}$ of the link should remain 0 and is not updated in the kinematic calibration process.

Table 4.3: Absolute measurement concept results using DH convention.

\begin{tabular}{rrrrr|rrrr}
\hline \multicolumn{4}{c}{ Joint Errors $[\mathrm{deg}]$} \\
\hline & $\delta \boldsymbol{\theta}_{s p}$ & $\delta \boldsymbol{\theta}_{i d}$ & $\delta \boldsymbol{\alpha}_{s p}$ & $\delta \boldsymbol{\alpha}_{i d}$ & $\delta \boldsymbol{a}_{s p}$ & $\delta \boldsymbol{a}_{i d}$ & $\delta \boldsymbol{d}_{s p}$ & $\delta \boldsymbol{d}_{i d}$ \\
\hline 0 & 16.6369 & 16.6369 & 16.2059 & 16.2059 & 27.8499 & -0.0000 & 13.1482 & 13.1482 \\
1 & 6.9619 & 6.9619 & 1.5040 & 1.5040 & 2.6125 & 2.6125 & -0.6683 & -0.6683 \\
2 & 1.2435 & 1.2435 & 1.6209 & 1.6209 & -4.7056 & -4.7056 & 0.8162 & 0.8162 \\
3 & 0.6355 & 0.6355 & 2.9723 & 2.9723 & -5.5328 & -5.5328 & 0.0000 & 0.0000 \\
4 & 1.8395 & 1.8395 & 2.9681 & 2.9681 & 0.8652 & 0.8652 & -3.6727 & -3.6727 \\
5 & -1.5833 & -1.5833 & -2.0665 & -2.0665 & -2.8527 & -2.8527 & -6.2984 & -6.2984 \\
6 & 3.7248 & 3.7248 & -2.5705 & -2.5705 & -2.7322 & -2.7322 & -1.1707 & -1.1707 \\
\hline \hline & \multicolumn{4}{c}{ Residual: $5.28 \times 10^{-06}$} \\
\hline
\end{tabular}

Table 4.4: Absolute measurement concept results using Hayati convention.

\begin{tabular}{rrrrr|rrrr}
\hline \multicolumn{4}{c}{ Joint Errors [deg] } \\
\hline & $\delta \boldsymbol{\theta}_{s p}$ & $\delta \boldsymbol{\theta}_{i d}$ & $\delta \boldsymbol{\alpha}_{s p}$ & $\delta \boldsymbol{\alpha}_{i d}$ & $\delta \boldsymbol{a}_{s p}$ & $\delta \boldsymbol{a}_{i d}$ & $\delta \boldsymbol{d}_{s p}$ & $\delta \boldsymbol{d}_{i d}$ \\
\hline 0 & 16.6369 & 16.6369 & 16.2059 & 16.2059 & 27.8499 & -0.0000 & 13.1482 & 13.1482 \\
1 & 6.9619 & 6.9619 & 1.5040 & 1.5040 & 2.6125 & 2.6125 & -0.6683 & -0.6683 \\
2 & 1.2435 & 1.2435 & 1.6209 & 1.6209 & -4.7056 & -4.7056 & 0.8162 & 0.8162 \\
3 & 0.6355 & 0.6355 & 2.9723 & 2.9723 & -5.5328 & -5.5328 & 0.0000 & 0.0000 \\
4 & 1.8395 & 1.8395 & 2.9681 & 2.9681 & 0.8652 & 0.8652 & -3.6727 & -3.6727 \\
5 & -1.5833 & -1.5833 & -2.0665 & -2.0665 & -2.8527 & -2.8527 & -6.2984 & -6.2984 \\
6 & 3.7248 & 3.7248 & -2.5705 & -2.5705 & -2.7322 & -2.7322 & -1.1707 & -1.1707 \\
\hline 3 & $\delta \beta_{s p}$ & 5.7983 & $\delta \beta_{i d}$ & 42.6681 & \multicolumn{5}{c}{ Residual: $5.02 \times 10^{-06}$} \\
\hline \hline
\end{tabular}




\section{Accuracy Assessment}

It is useful to define a certain measure of accuracy for confirming the improvement of the A465 accuracy from kinematic calibration. The accuracy on the end-effector pose was defined as the RMS values of the absolute errors in Eq. (2.2). These accuracies were computed for each pose, and they are plotted in Figures 4.3(a) and 4.3(b). Note that the dotted line represents the mean and the dashed lines represent the standard deviation from the mean of the data set.
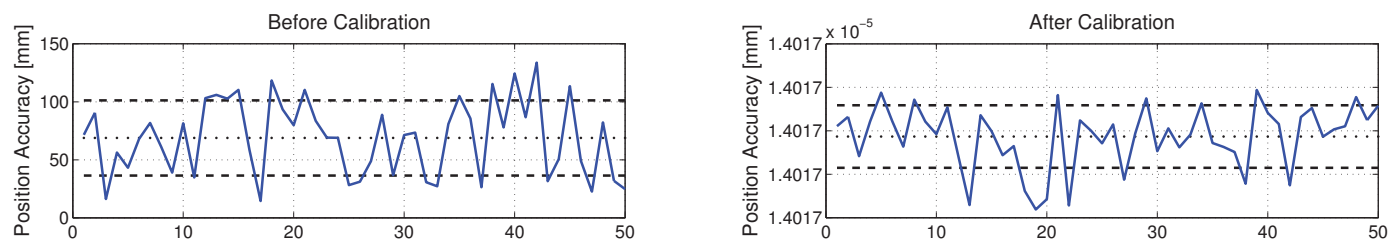

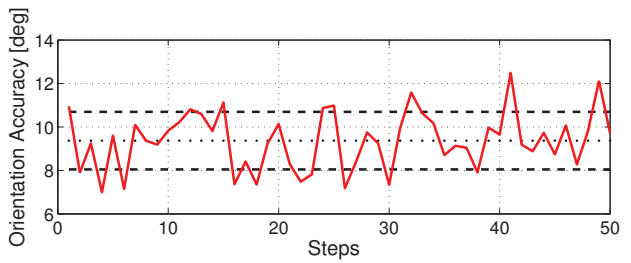

(a)

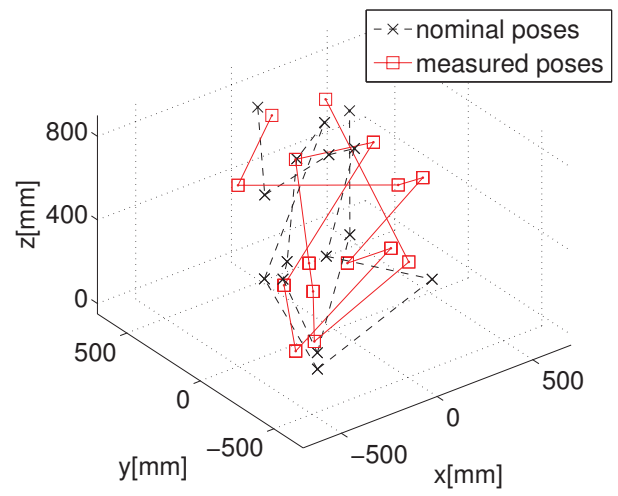

(c)

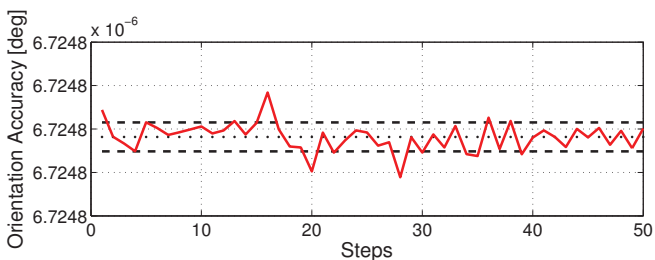

(b)

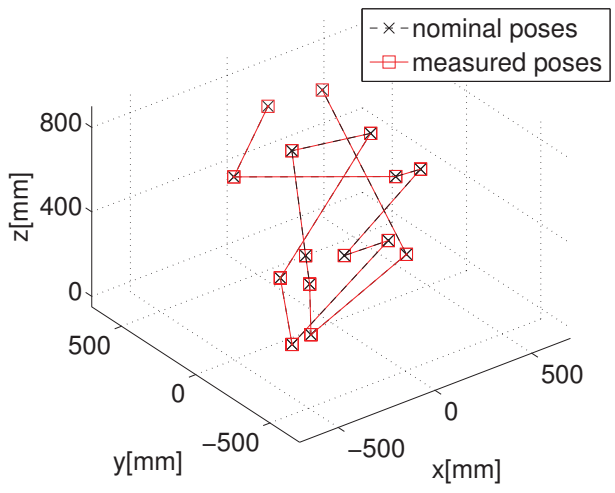

(d)

Figure 4.3: The (a) absolute accuracy and (b) relative accuracy after AMC calibration. The corresponding first 15 absolute and relative positions are shown in (c) and (d), respectively. 
In the simulation, 50 poses were generated to perform the kinematic calibration, but only the first 15 absolute and relative poses are shown in Figures 4.3(c) and 4.3(d) for clarity. From all the subplots in Figure 4.3, a great deal of inaccuracies in the poses were observed given that the deviations in the kinematic parameters were the only contribution. The subfigures provide one with an idea of the absolute accuracy for each pose, but the overall improvement of the accuracy can be compared using the RMS values. The overall absolute accuracy improved from roughly $\pm 10.738 \mathrm{~mm}$ and $\pm 0.194^{\circ}$ to roughly $\pm 1.982 \times 10^{-6} \mathrm{~mm}$ and $\pm 0.000^{\circ}$, respectively.

\subsubsection{Relative Measurement Results}

Using the same simulated $\boldsymbol{\zeta}_{A}$ as before, the simulation here presents the kinematic calibration using the RMC. The solution of this simulation is also identified using SVD, the results are summarized in Table 4.5. It took 17 iterations to converge to a solution with residual smaller than the specified tolerance. However, only 24 out of 28 parameters were identified correctly. The mis-identified parameter $\delta \beta_{3}$ was an

Table 4.5: Relative measurement concept results.

\begin{tabular}{rrrrr|rrrr}
\hline \multicolumn{3}{c}{ Joint Errors [deg] } \\
\hline & $\delta \boldsymbol{\theta}_{s p}$ & $\delta \boldsymbol{\theta}_{i d}$ & $\delta \boldsymbol{\alpha}_{s p}$ & $\delta \boldsymbol{\alpha}_{i d}$ & $\delta \boldsymbol{a}_{s p}$ & $\delta \boldsymbol{a}_{i d}$ & $\delta \boldsymbol{d}_{s p}$ & $\delta \boldsymbol{d}_{i d}$ \\
\hline 0 & 16.6369 & 16.6369 & 16.2059 & 16.2059 & 27.8499 & -0.0000 & 13.1482 & 0.0000 \\
1 & 6.9619 & 6.9619 & 1.5040 & 1.5040 & 2.6125 & 2.6125 & -0.6683 & 0.0000 \\
2 & 1.2435 & 1.2435 & 1.6209 & 1.6209 & -4.7056 & -4.7056 & 0.8162 & 0.8162 \\
3 & 0.6355 & 0.6355 & 2.9723 & 2.9723 & -5.5328 & -5.5328 & 0.0000 & 0.0000 \\
4 & 1.8395 & 1.8395 & 2.9681 & 2.9681 & 0.8652 & 0.8652 & -3.6727 & -3.6727 \\
5 & -1.5833 & -1.5833 & -2.0665 & -2.0665 & -2.8527 & -2.8527 & -6.2984 & -6.2984 \\
6 & 3.7248 & 3.7248 & -2.5705 & -2.5705 & -2.7322 & -2.7322 & -1.1707 & -1.1707 \\
\hline 3 & $\delta \beta_{s p}$ & 5.7983 & $\delta \beta_{i d}$ & 42.6681 & \multicolumn{5}{c}{ Residual: $9.28 \times 10^{-06}$} \\
\hline \hline
\end{tabular}


expected outcome, but the other three parameters, $\delta a_{b}, \delta d_{b}$, and $\delta d_{1}$, affected only the translational placement of the manipulator, and they are unobservable using RMC calibration.

\section{Accuracy Assessment}

Using the measure of accuracy defined previously, the absolute accuracy of each pose is presented in Figure 4.4(a). Notice that the absolute position accuracy after calibration
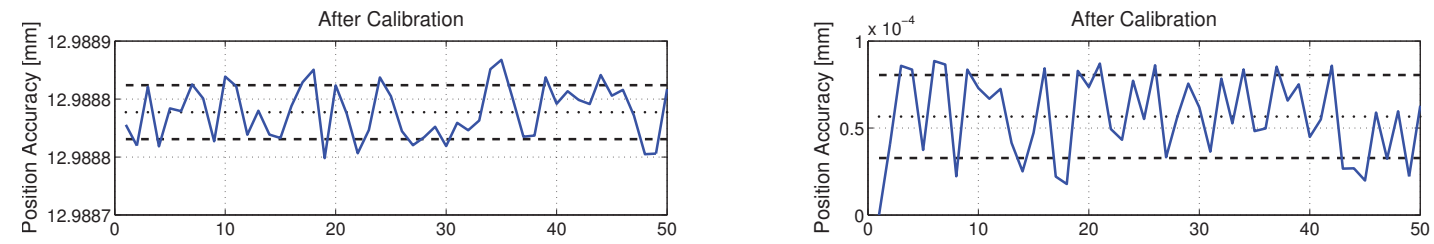

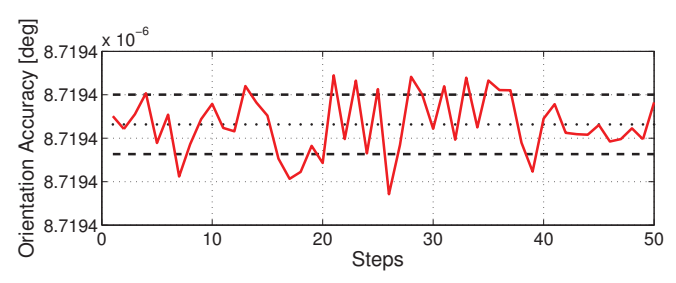

(a)

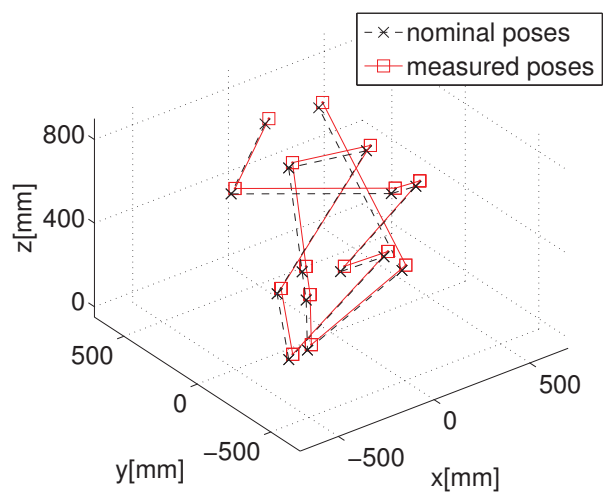

(c)

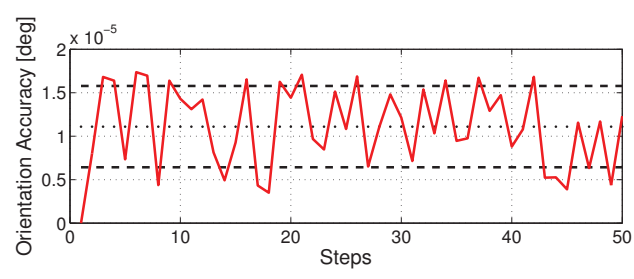

(b)

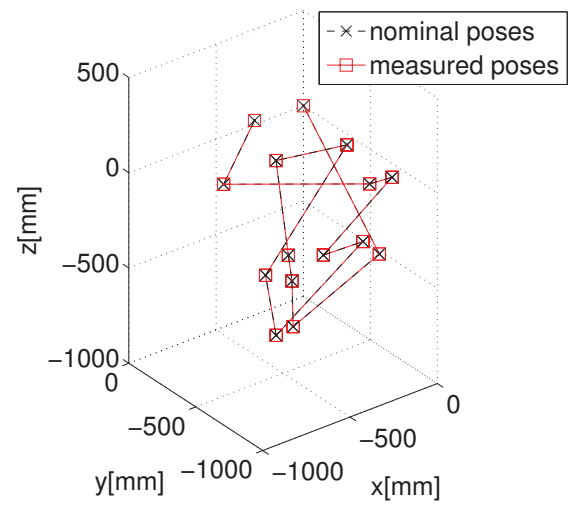

(d)

Figure 4.4: The (a) absolute accuracy and (b) relative accuracy after RMC calibration. The corresponding first 15 absolute and relative positions are shown in (c) and (d), respectively. 
here is shifted to a higher mean than the one shown in Figure 4.3(b), while the absolute orientation accuracy remains low. This is because the actual joint 1 location is unobservable, and it is located using the nominal parameters instead.

However, instead of relying on the absolute accuracy, the RMS error of the relative errors in Eq. (2.6) defines the relative accuracy. These relative accuracies are computed for each pose, and are plotted in Figure 4.4(b). The relative accuracy of the first pose is obviously 0, because the RMC used the first pose as the starting reference, meaning that the errors of all other poses were expressed relative to the previous one.

Again, 50 poses were generated in the simulation, but only the first 15 absolute poses are presented in Figure 4.4(c), and the first 15 relative poses are presented in Figure 4.4(d). It is shown that there is a slight translational shift in the overall absolute poses as expected, and the relative poses converged perfectly. The improvement in the end-effector accuracy using RMC can be compared using the RMS values as well. The overall absolute accuracy improved from $\pm 10.738 \mathrm{~mm}$ and $\pm 0.194^{\circ}$ to $\pm 1.4392 \mathrm{~mm}$ and $\pm 0.000^{\circ}$, respectively. The overall relative accuracy on the other hand improved from $\pm 12.831 \mathrm{~mm}$ and $\pm 0.509^{\circ}$ to $\pm 8.852 \times 10^{-6} \mathrm{~mm}$ and $\pm 0.000^{\circ}$, respectively.

\subsection{Observation Strategy}

The ability to accurately identify kinematic parameters is affected by the measurement noise, the number of measurements, and the measurement pose selection. The condition number $\kappa(\mathbf{J})$, defined in Chapter 2, is used to quantify the goodness of the estimation. 


\subsubsection{Conditioning the Jacobian Matrix}

In Chapter 2, the condition number $\kappa(\mathbf{J})$ is defined using the singular values of $\mathbf{J}$. In kinematic calibration, $\kappa(\mathbf{J})$ can be really high even without the measurement noise $[7,12]$. This is because at least 24 parameters (for a standard $6 \mathrm{R}$ serial manipulator) need to be identified, leading to some parameters being linearly independent in some configurations and dependent in others.

From Table 4.5, it was established that four parameters were not identified correctly: $\delta a_{b} ; \delta d_{b} ; \delta d_{1}$; and $\delta \beta_{3}$. Unlike $\delta \beta_{3}$, the other three parameters firmly defined the kinematic relationship between two frames, and they still contributed to the singularity of $\mathbf{J}$. Because the parameters, $\delta a_{b}, \delta d_{b}, \delta d_{1}$, are unobservable with the RMC, the estimated results for the corresponding parameters are not reliable. Therefore, the columns of $\mathbf{J}$ associated with those parameters, $\delta a_{b}, \delta d_{b}$, and $\delta d_{1}$, should all be removed for computing $\kappa(\mathbf{J})$.

\subsubsection{Measurement Noise}

Like all real world problems, as long as measurements are made, there exists some noise that cannot be completely accounted for with the mathematical model of the problem. Many simulations were performed to observe the effect of measurement noise on the outcome of the kinematic calibration results.

For simplicity, the simulations for this section were written by extending the AMC simulation from the previous section with the inclusion of assumed measurement noise. As mentioned, the noise is random, but can be approximated by assuming a zero mean Gaussian distribution. The outcomes observed in the simulations performed here are also compared to the results of the no noise situation presented in Figures 4.3(a), 4.3(b), 4.3(c), and 4.3(d). 


\section{Input Noise Only}

In practical kinematic calibration problems, the joint angles used in the kinematic model are measured directly using the joint transducers. The errors between the joint transducers' reading and the actual joint angles configuring the manipulator constitute the input noise, and is generally small and nondeterministic. This input noise is defined with the standard deviation of $\bar{\sigma}_{\boldsymbol{\theta}}=10^{-3} \mathrm{rad}$ in the simulation, and the resulting absolute accuracy is presented in Figures 4.5(a) and 4.5(b).
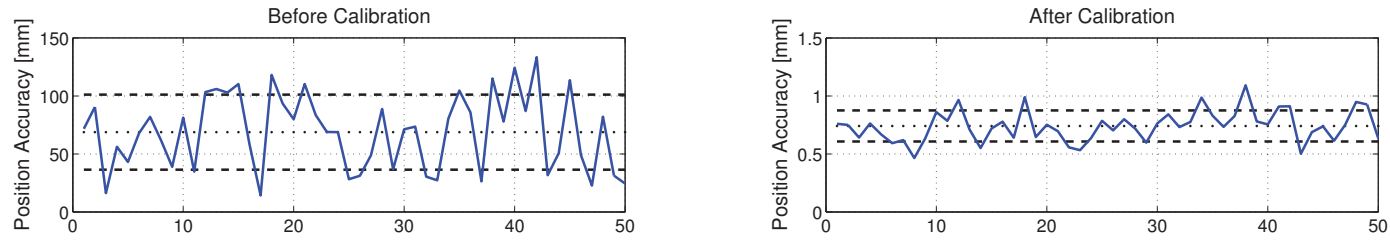

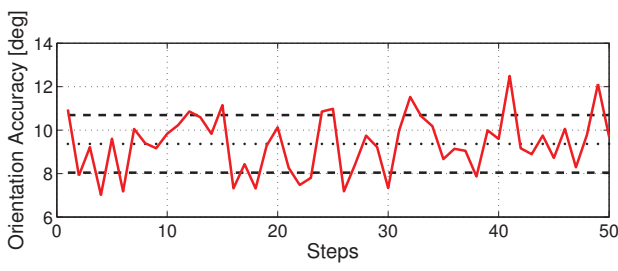

(a)

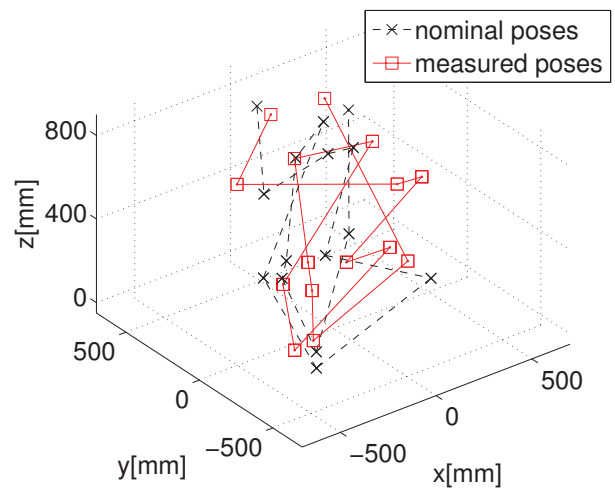

(c)

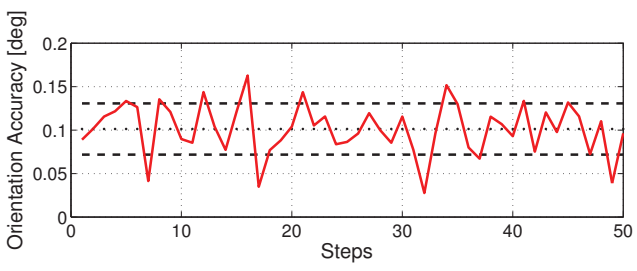

(b)

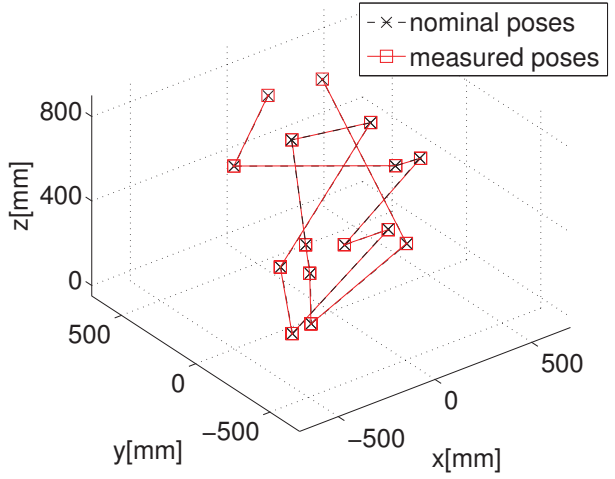

(d)

Figure 4.5: Simulation including only the input noise. The absolute accuracy (a) before and (b) after AMC calibration. The first 15 absolute position before and after AMC calibration are shown in (c) and (d), respectively. 
After AMC, the absolute accuracy of the pose is still greatly improved. The overall accuracy improved from roughly $\pm 10.73 \mathrm{~mm}$ and $\pm 0.194^{\circ}$ to roughly $\pm 0.107 \mathrm{~mm}$ and $\pm 0.002^{\circ}$, respectively. As expected with the presence of measurement noise, although the results of kinematic calibration improved the accuracy, it cannot be better than the existing measurement noise.

\section{Output Noise Only}

The most difficult task in kinematic calibration is the measurement of the end-effector pose, which requires an external measurement system to get all components of the pose. The errors observed between the measurement system and the actual placement of the end-effector pose are the output noise. The output noise is defined with two standard deviations, $\bar{\sigma}_{\mathbf{p}}=10 \mathrm{~mm}$ and $\bar{\sigma}_{\boldsymbol{\delta}}=10^{-5} \mathrm{rad}\left(5.730 \times 10^{-4} \mathrm{deg}\right.$. $)$ in the simulation, and the absolute accuracy is presented in Figures 4.6(a) and 4.6(b), respectively.

After AMC, the overall accuracy improved from roughly $\pm 10.7891 \mathrm{~mm}$ and $\pm 0.194^{\circ}$ to roughly $\pm 1.289 \mathrm{~mm}$ and $\pm 0.001^{\circ}$, respectively. The first 15 end-effector poses before and after calibration are presented in Figures 4.6(c) and 4.6(d), respectively. From these figures, the orientation of the manipulator seemed to have been corrected nicely, but there are still existing deviations in the end-effector positions.

\section{Input and Output Noise}

In practice, there is both input and output measurement noise involved in the kinematic calibration process. It is impossible to distinguish between input and output noise, and thus the identification results are affected by both. 

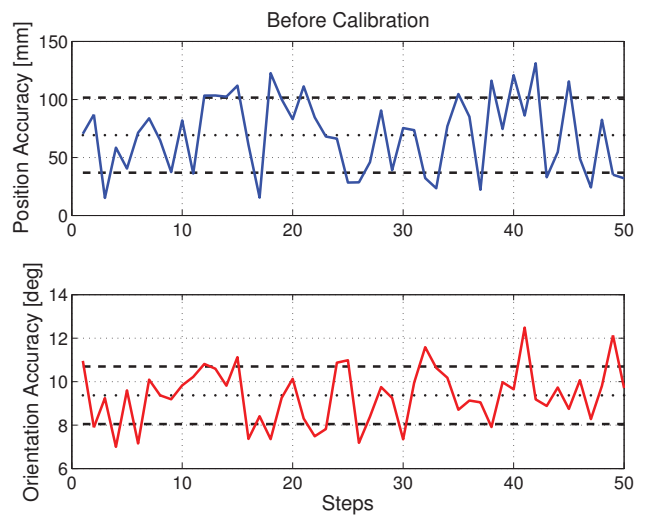

(a)

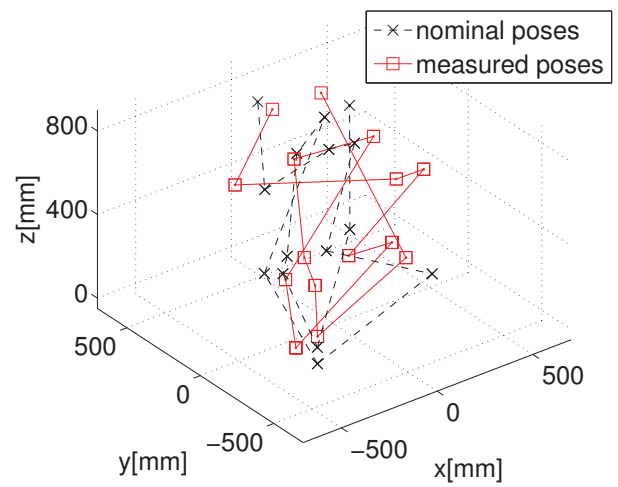

(c)
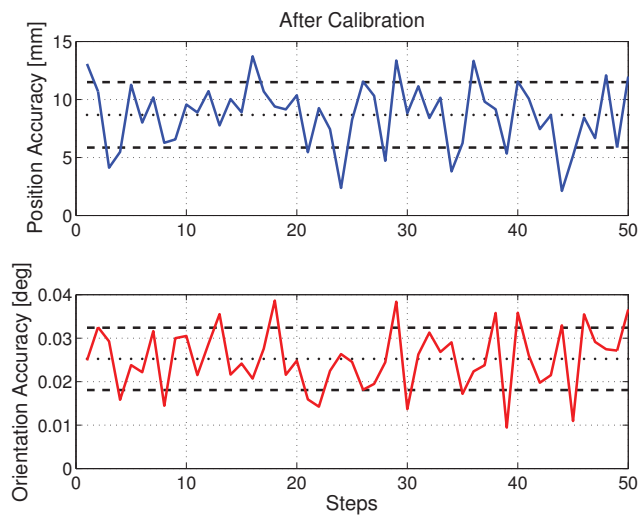

(b)

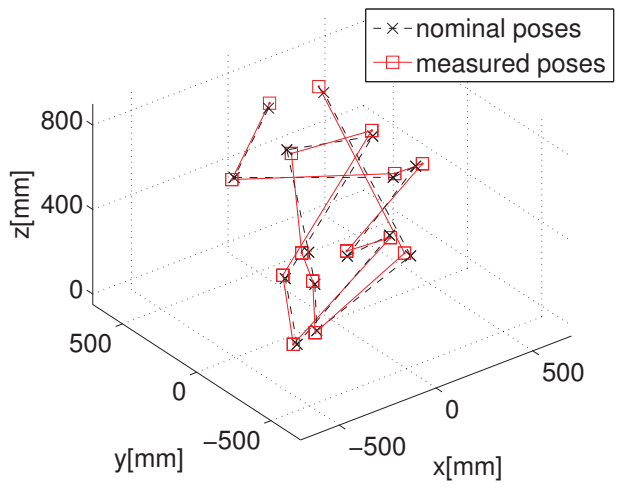

(d)

Figure 4.6: Simulation including only the output measurement noise. The absolute accuracy (a) before and (b) after AMC calibration. The first 15 absolute positions before and after AMC calibration are shown in (c) and (d), respectively.

For the simulation, the input noise is defined with $\bar{\sigma}_{\boldsymbol{\theta}}=10^{-3} \mathrm{rad}$, and the output noise is defined with $\bar{\sigma}_{\mathbf{p}}=10 \mathrm{~mm}$ and $\bar{\sigma}_{\boldsymbol{\delta}}=10^{-5} \mathrm{rad}$. The input noise is added to the joint angles to define the nominal kinematic model, then the output noise is added to the end-effector poses computed using the model. The absolute accuracy of every pose before and after the AMC calibration are presented in Figures 4.7(a) and 4.7(b), respectively. 
After performing AMC calibration, the overall accuracy in this situation improved from roughly $\pm 10.784 \mathrm{~mm}$ and $\pm 0.194^{\circ}$ to roughly $\pm 1.362 \mathrm{~mm}$ and $\pm 0.002^{\circ}$, respectively. The first 15 end-effector poses before and after calibration are presented in Figures $4.7(\mathrm{c})$ and $4.7(\mathrm{~d})$, respectively. From these figures, the end-effector poses converged to the same level as the case with the output noise only. This implies that the level of input noise in the simulation does not have as much of an impact to the identification results as the output noise.
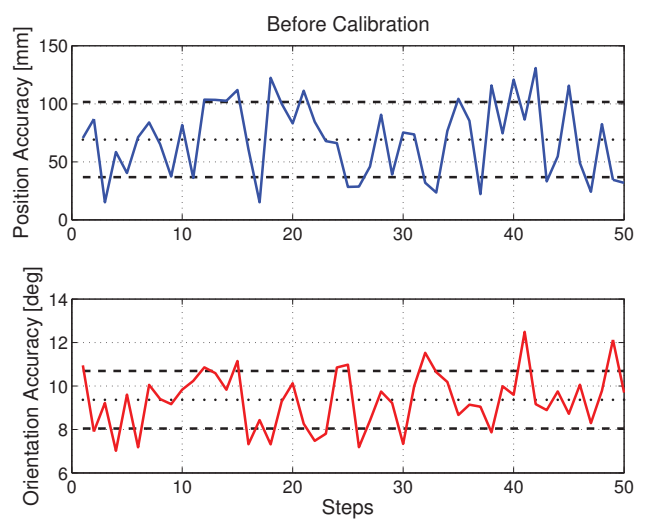

(a)

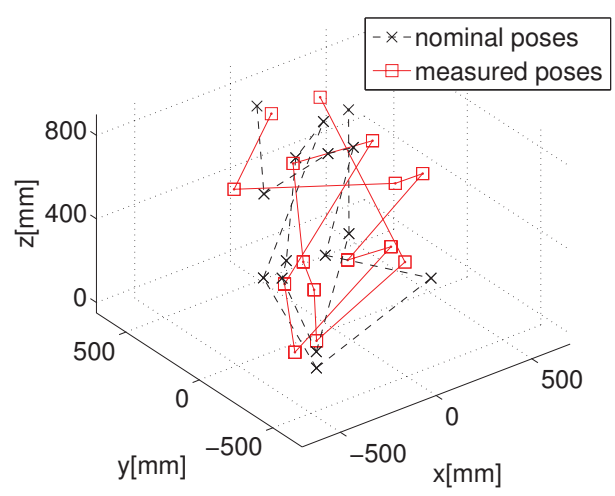

(c)
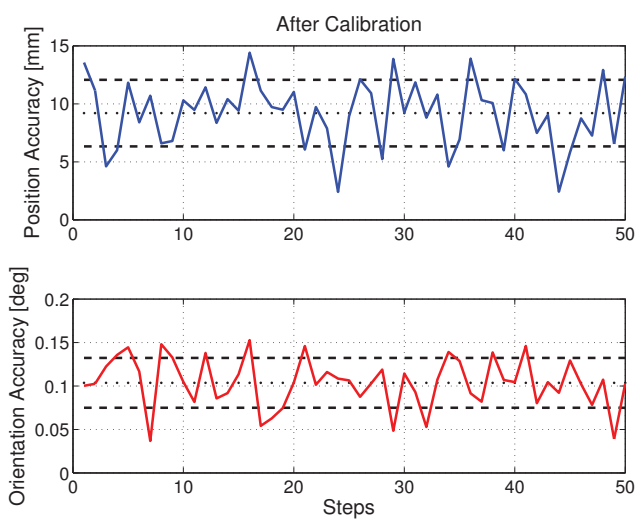

(b)

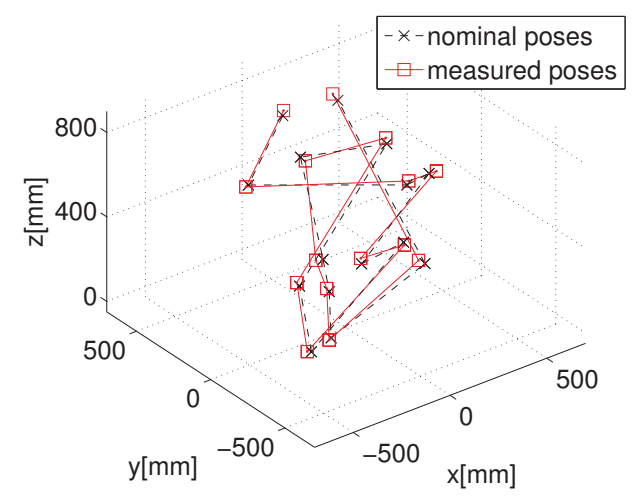

(d)

Figure 4.7: Simulations including both the input and output measurement noise. The absolute accuracy (a) before and (b) after calibration. The first 15 absolute poses before and after calibration are shown in (c) and (d), respectively. 
From the simulations, some observations can be made on the effect of measurement noise on the outcome of the kinematic calibration results using AMC. Similar observations were found with the simulations for RMC, and thus only one method is presented here. All forms of noise are shown to be unable to reduce the residuals below the norm of the specified measurement noise to reach the specified tolerance. Even though the algorithm residual may be orders of magnitude above the specified tolerance, based on the end-effector poses in Figures 4.5(d), 4.6(d), and 4.7(d), the calibrated nominal poses still converge well to the simulated actual poses.

\section{The Effect of Kinematic Model Convention}

As previously discussed, there are two forms of kinematic modelling convention used in this thesis. The effects between the conventions have not yet been concretely made. The impact of the kinematic modelling convention between the DH and Hayati conventions is revisited here with the presence of input and output noise in the kinematic calibration.

Many simulations were performed to see the effect of the kinematic modelling convention when the input and output measurement noise is present in the kinematic calibration. The same outcomes were observed in all simulations, and thus only one representative case is presented in Tables 4.6 and 4.7 .

The simulations were setup to be the same as the ones used to investigate the effect of measurement noise on the kinematic calibration results. The same standard deviations of input and output measurement noise were used $\left(\bar{\sigma}_{\boldsymbol{\theta}}=10^{-3} \mathrm{rad}, \bar{\sigma}_{\mathbf{p}}=\right.$ $10 \mathrm{~mm}$, and $\bar{\sigma}_{\boldsymbol{\delta}}=10^{-5} \mathrm{rad}$ ), the joint angles and the simulated $\boldsymbol{\zeta}_{A}$ (in DH and Hayati

conventions) are defined to be the same for each simulation. All parameters were identified to be really close between both conventions except for $\delta d_{2}$ and $\delta d_{3}$. Looking 
Table 4.6: AMC using DH with the presence of both input and output noise.

\begin{tabular}{rrrrr|rrrr}
\hline \multicolumn{3}{c}{ Joint Errors $[\mathrm{deg}]$} \\
\hline & $\delta \boldsymbol{\theta}_{s p}$ & $\delta \boldsymbol{\theta}_{i d}$ & $\delta \boldsymbol{\alpha}_{s p}$ & $\delta \boldsymbol{\alpha}_{i d}$ & $\delta \boldsymbol{a}_{s p}$ & $\delta \boldsymbol{a}_{i d}$ & $\delta \boldsymbol{d}_{s p}$ & $\delta \boldsymbol{d}_{i d}$ \\
\hline 0 & 16.6369 & 16.5297 & 16.2059 & 16.1647 & 27.8499 & 25.7481 & 13.1482 & 11.1478 \\
1 & 6.9619 & 7.1017 & 1.5040 & 1.4656 & 2.6125 & 4.5524 & -0.6683 & 2.9213 \\
2 & 1.2435 & 0.8017 & 1.6209 & 1.6181 & -4.7056 & -5.7248 & 0.8162 & 72.6842 \\
3 & 0.6355 & 1.0768 & 2.9723 & 2.9651 & -5.5328 & -7.5951 & 0.0000 & -72.6805 \\
4 & 1.8395 & 1.8435 & 2.9681 & 2.9783 & 0.8652 & 3.1903 & -3.6727 & -2.0999 \\
5 & -1.5833 & -1.5912 & -2.0665 & -2.0847 & -2.8527 & -4.8232 & -6.2984 & -5.9813 \\
6 & 3.7248 & 3.4564 & -2.5705 & -2.5740 & -2.7322 & -4.3757 & -1.1707 & -26.1719 \\
\hline \hline
\end{tabular}

Table 4.7: AMC using MDH with the presence of both input and output noise.

\begin{tabular}{rrrrr|rrrr}
\hline \multicolumn{3}{c}{ Joint Errors [deg] } \\
\hline & $\delta \boldsymbol{\theta}_{s p}$ & $\delta \boldsymbol{\theta}_{i d}$ & $\delta \boldsymbol{\alpha}_{s p}$ & $\delta \boldsymbol{\alpha}_{i d}$ & $\delta \boldsymbol{a}_{s p}$ & $\delta \boldsymbol{a}_{i d}$ & $\delta \boldsymbol{d}_{s p}$ & $\delta \boldsymbol{d}_{i d}$ \\
\hline 0 & 16.6369 & 16.5298 & 16.2059 & 16.1647 & 27.8499 & 25.7481 & 13.1482 & 11.1461 \\
1 & 6.9619 & 7.1017 & 1.5040 & 1.4656 & 2.6125 & 4.5520 & -0.6683 & 2.9227 \\
2 & 1.2435 & 1.1972 & 1.6209 & 1.6181 & -4.7056 & -5.7174 & 0.8162 & 0.0325 \\
3 & 0.6355 & 0.6814 & 2.9723 & 2.9651 & -5.5328 & -7.5955 & 0.0000 & 0.0000 \\
4 & 1.8395 & 1.8435 & 2.9681 & 2.9783 & 0.8652 & 3.1901 & -3.6727 & -2.1000 \\
5 & -1.5833 & -1.5912 & -2.0665 & -2.0847 & -2.8527 & -4.8231 & -6.2984 & -5.9812 \\
6 & 3.7248 & 3.4565 & -2.5705 & -2.5740 & -2.7322 & -4.3747 & -1.1707 & -26.1647 \\
\hline 3 & $\delta \beta_{s p}$ & 4.2239 & $\delta \beta_{i d}$ & -1.6191 & & & & \\
\hline \hline
\end{tabular}

at the results for the DH convention, the parameters $\delta d_{2}$ and $\delta d_{3}$ are unusually high (sometimes the magnitudes of the parameters approached the unit of $\mathrm{m}$ ) compared to the other identified parameters. The two parameters appeared to show dependencies on each other. However, in the case of the Hayati convention, by constantly setting $d_{3}=0$, the linear dependencies between the parameters vanish and the result is a better identified $\delta d_{2}$. 


\subsubsection{Number of Measurement Poses}

As previously discussed, the identified kinematic parameters are generally affected by the presence of measurement noise, but the identified results can be improved by increasing the number of measurement poses. Figure 4.8 was made to see the effect of increasing the number of measurements to the goodness of identification using $\kappa(\mathbf{J})$.

In this simulation, 100 poses were generated by randomly choosing the joint angles within the manipulator's joint limits, just like all the previously performed simulations. These joint angles and $\boldsymbol{\zeta}_{A}$ are specified from the start of the simulation program, and they do not change throughout the analysis. The number of poses in the figure are extracted from the 100 randomly generated poses, where $N$ poses referred to the first $N$ poses stored in the 100 poses. Therefore, the simulation analysis is strictly on varying the number of poses only.

From the figure, the first point is set for 5 measurement poses, because there are 28 parameters to be identified and 6 independent equations were produced per pose, resulting in at least 5 poses (30 equations) required for identification. There is a diminishing return in this relationship, the $\kappa(\mathbf{J})$ becomes steady after 40 measurements.

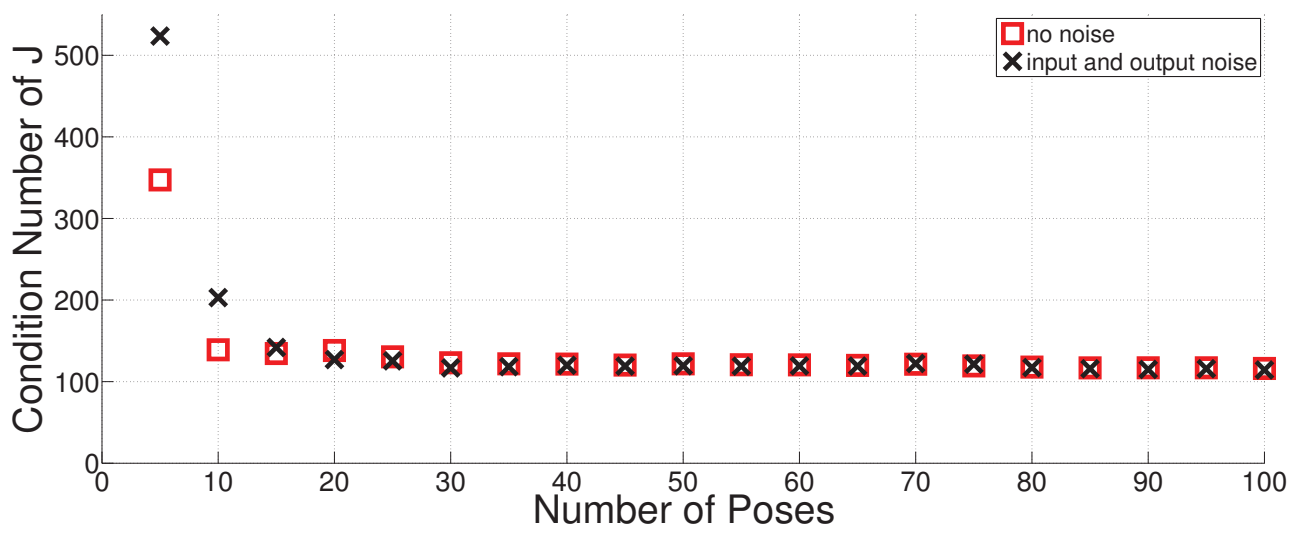

Figure 4.8: The number of poses verses the condition number of the Jacobian. 


\subsubsection{Pose Selection}

The success of identifying all the kinematic parameters can also be affected by the selected poses for measurement, because some parameters are unobservable in some poses. The pose selections can also be restricted depending on the measurement devices.

In this section, some restricted and organized trajectories that can be used in kinematic calibration are analyzed. For the analyses, because $\boldsymbol{\zeta}_{A}$ is simulated using Eq. (4.1), 30 iterations of $\mathrm{AMC}$ and $\mathrm{RMC}$ were run for each specified pose trajectory to see the changes in the results for each trajectory. The terminating condition of the kinematic calibration algorithms (AMC and RMC) are defined to be res $\leq 10^{-6}$ or $n_{i t e r}=50$, whichever criteria is met first.

\section{Straight Line Trajectories}

Six different straight line trajectories of the end-effector, presented in Figure 4.9, were analyzed. The first three, $\mathrm{L}=(\mathrm{a}),(\mathrm{b}),(\mathrm{c})$, are along the $\mathbf{x}, \mathbf{y}$, and $\mathbf{z}$-axes. The next three, $\mathrm{L}=(\mathrm{d}),(\mathrm{e}),(\mathrm{f})$, are diagonal lines on the $\mathbf{x y}, \mathbf{x z}$, and $\mathbf{y z}$-planes. The endeffector is oriented in three different ways $(\mathrm{T}=1,2,3)$, where the $\mathbf{z}$-axis of $\Sigma_{E E}$ is pointed normal to the $\mathbf{y z}, \mathbf{x z}$, and $\mathbf{x y}$-planes, respectively.

These straight line trajectories were expressed and programmed in terms of the Cartesian space, and the joint angles to configure these poses were computed using inverse kinematics. The simulation was run to analyze the effect of straight line trajectories on the kinematic calibration with AMC and RMC. 


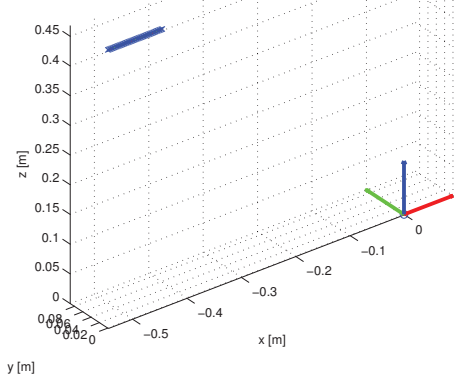

(a)

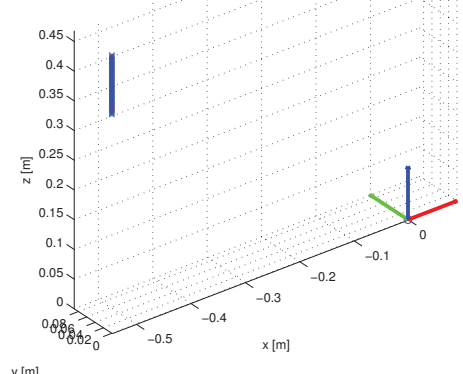

(c)

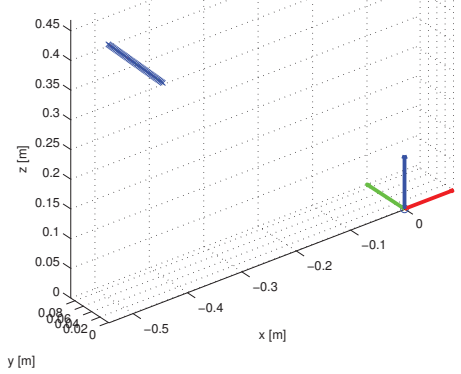

(e)

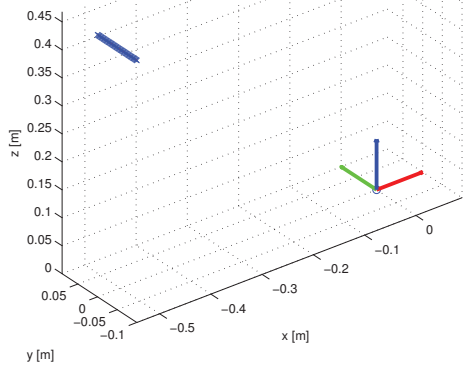

(b)

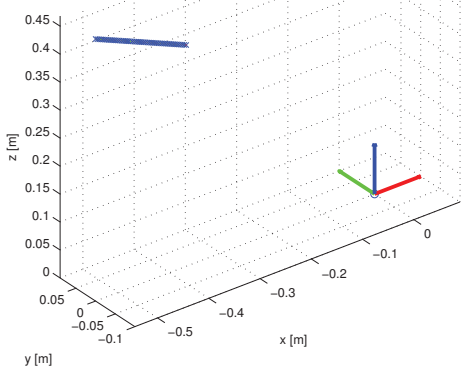

(d)

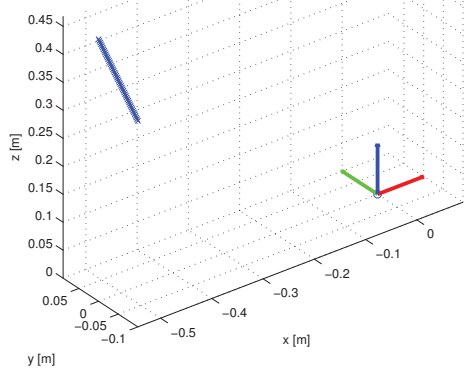

(f)

Figure 4.9: Six varieties of straight line trajectories of end-effector pose. 
Table 4.8: Absolute measurement concept results using straight line trajectories.

\begin{tabular}{|c|c|c|c|c|c|c|c|}
\hline \multicolumn{8}{|c|}{ Absolute Measurement Concept } \\
\hline $\mathrm{L}$ & $\mathrm{T}$ & $n_{\text {iter }}$ & res & $\operatorname{rank}(\mathbf{J})$ & $\kappa(\mathbf{J})$ & $\delta_{p}[\mu \mathrm{m}]$ & $\delta_{r}[\mu \mathrm{rad}]$ \\
\hline \multirow[t]{3}{*}{ (a) } & 1 & 16 & $6.703 \times 10^{-06}$ & $18 / 28$ & $3.3 \times 10^{+16}$ & 0.907 & 3.2 \\
\hline & 2 & 18 & $6.557 \times 10^{-06}$ & $28 / 28$ & $2.2 \times 10^{+09}$ & 3.15 & 0.93 \\
\hline & 3 & 16 & $7.656 \times 10^{-06}$ & $18 / 28$ & $3.4 \times 10^{+16}$ & 1.13 & 3.6 \\
\hline \multirow[t]{3}{*}{ (b) } & 1 & 50 & $3.117 \times 10^{+06}$ & $25 / 28$ & $1.4 \times 10^{+15}$ & $4.08 \times 10^{+15}$ & $3.0 \times 10^{+06}$ \\
\hline & 2 & 17 & $7.371 \times 10^{-06}$ & $28 / 28$ & $3.0 \times 10^{+09}$ & 0.255 & 3.7 \\
\hline & 3 & 50 & $2.087 \times 10^{+06}$ & $22 / 28$ & $3.7 \times 10^{+21}$ & $3.02 \times 10^{+14}$ & $3.3 \times 10^{+06}$ \\
\hline \multirow[t]{3}{*}{ (c) } & 1 & 17 & $5.310 \times 10^{-06}$ & $18 / 28$ & $3.2 \times 10^{+16}$ & 0.874 & 2.5 \\
\hline & 2 & 20 & $6.562 \times 10^{-06}$ & $24 / 28$ & $2.7 \times 10^{+16}$ & 2.91 & 1.5 \\
\hline & 3 & 14 & $9.928 \times 10^{-06}$ & $18 / 28$ & $3.3 \times 10^{+16}$ & 4.04 & 2.8 \\
\hline \multirow[t]{3}{*}{ (d) } & 1 & 17 & $6.548 \times 10^{-06}$ & $28 / 28$ & $2.6 \times 10^{+08}$ & 0.023 & 3.3 \\
\hline & 2 & 17 & $7.506 \times 10^{-06}$ & $28 / 28$ & $7.6 \times 10^{+08}$ & 0.405 & 3.7 \\
\hline & 3 & 17 & $5.966 \times 10^{-06}$ & $24 / 28$ & $1.9 \times 10^{+16}$ & 0.526 & 2.9 \\
\hline \multirow[t]{3}{*}{ (e) } & 1 & 17 & $6.458 \times 10^{-06}$ & $18 / 28$ & $4.9 \times 10^{+16}$ & 0.129 & 3.2 \\
\hline & 2 & 20 & $9.373 \times 10^{-06}$ & $28 / 28$ & $1.0 \times 10^{+09}$ & 4.57 & 1.0 \\
\hline & 3 & 15 & $7.648 \times 10^{-06}$ & $18 / 28$ & $3.3 \times 10^{+16}$ & 1.94 & 3.3 \\
\hline \multirow[t]{3}{*}{ (f) } & 1 & 17 & $6.135 \times 10^{-06}$ & $28 / 28$ & $2.3 \times 10^{+08}$ & 0.429 & 3.0 \\
\hline & 2 & 50 & 1.449 & $28 / 28$ & $1.3 \times 10^{+10}$ & $6.59 \times 10^{+08}$ & $1.3 \times 10^{+06}$ \\
\hline & 3 & 17 & $8.884 \times 10^{-06}$ & $24 / 28$ & $1.9 \times 10^{+16}$ & 1.56 & 4.1 \\
\hline
\end{tabular}

Tables 4.8 and 4.9 summarize the first iteration of the straight line trajectories simulation using AMC and RMC, respectively. Based on all the simulations performed this far, the full rank of $\mathbf{J}$ is determined to be 28 or 25 for AMC or RMC, respectively, if the A465 is setup using the Hayati convention. From both tables, there are some trajectories leading to full rank $\mathbf{J}$ with convergence, while others produced full rank $\mathbf{J}$ with diverged solutions, rank deficient $\mathbf{J}$ with converged solutions, or rank deficient $\mathbf{J}$ 
Table 4.9: Relative measurement concept results using straight line trajectories.

\begin{tabular}{|c|c|c|c|c|c|c|c|}
\hline \multicolumn{8}{|c|}{ Relative Measurement Concept } \\
\hline $\mathrm{L}$ & $\mathrm{T}$ & $n_{\text {iter }}$ & res & $\operatorname{rank}(\mathbf{J})$ & $\kappa(\mathbf{J})$ & $\delta_{p}\left[10^{3} \mu \mathrm{m}\right]$ & $\delta_{r}[\mu \mathrm{rad}]$ \\
\hline \multirow[t]{3}{*}{ (a) } & 1 & 3 & $7.054 \times 10^{-06}$ & $15 / 25$ & $1.0 \times 10^{+21}$ & 62.4 & $1.4 \times 10^{+02}$ \\
\hline & 2 & 7 & $8.322 \times 10^{-06}$ & $25 / 25$ & $7.7 \times 10^{+08}$ & 5.13 & 4.4 \\
\hline & 3 & 7 & $6.689 \times 10^{-06}$ & $15 / 25$ & $2.2 \times 10^{+20}$ & 63.3 & 8.4 \\
\hline \multirow[t]{3}{*}{ (b) } & 1 & 50 & $1.232 \times 10^{+05}$ & $22 / 25$ & $7.4 \times 10^{+15}$ & $1.24 \times 10^{+10}$ & $6.9 \times 10^{+03}$ \\
\hline & 2 & 50 & $1.815 \times 10^{+02}$ & $24 / 25$ & $2.9 \times 10^{+13}$ & $1.89 \times 10^{+09}$ & $5.4 \times 10^{+03}$ \\
\hline & 3 & 50 & $1.998 \times 10^{+04}$ & $20 / 25$ & $1.9 \times 10^{+20}$ & $2.09 \times 10^{+10}$ & $3.0 \times 10^{+03}$ \\
\hline \multirow[t]{3}{*}{ (c) } & 1 & 6 & $7.802 \times 10^{-06}$ & $15 / 25$ & $9.4 \times 10^{+20}$ & 61.8 & $1.8 \times 10^{+01}$ \\
\hline & 2 & 50 & $3.593 \times 10^{+04}$ & $17 / 25$ & $9.3 \times 10^{+27}$ & $2.31 \times 10^{+09}$ & $5.7 \times 10^{+03}$ \\
\hline & 3 & 50 & $1.612 \times 10^{+04}$ & $15 / 25$ & $1.9 \times 10^{+27}$ & $5.44 \times 10^{+09}$ & $4.6 \times 10^{+02}$ \\
\hline \multirow[t]{3}{*}{$(\mathrm{d})$} & 1 & 11 & $5.411 \times 10^{-06}$ & $25 / 25$ & $2.2 \times 10^{+07}$ & 4.78 & $1.3 \times 10^{-01}$ \\
\hline & 2 & 50 & $1.065 \times 10^{+03}$ & $23 / 25$ & $4.3 \times 10^{+16}$ & $3.62 \times 10^{+08}$ & $1.6 \times 10^{+03}$ \\
\hline & 3 & 6 & $5.280 \times 10^{-06}$ & $21 / 25$ & $2.9 \times 10^{+14}$ & 5.15 & 8.6 \\
\hline \multirow[t]{3}{*}{ (e) } & 1 & 6 & $7.062 \times 10^{-06}$ & $15 / 25$ & $9.6 \times 10^{+19}$ & 62.6 & $1.8 \times 10^{+01}$ \\
\hline & 2 & 50 & $3.790 \times 10^{+03}$ & $23 / 25$ & $8.2 \times 10^{+13}$ & $4.57 \times 10^{+09}$ & $4.8 \times 10^{+02}$ \\
\hline & 3 & 8 & $7.330 \times 10^{-06}$ & $15 / 25$ & $5.5 \times 10^{+20}$ & 62.4 & 4.3 \\
\hline \multirow[t]{3}{*}{ (f) } & 1 & 12 & $7.171 \times 10^{-06}$ & $25 / 25$ & $8.7 \times 10^{+06}$ & 6.22 & $6.1 \times 10^{-02}$ \\
\hline & 2 & 50 & $5.992 \times 10^{+01}$ & $25 / 25$ & $3.4 \times 10^{+09}$ & $9.29 \times 10^{+07}$ & $6.0 \times 10^{+03}$ \\
\hline & 3 & 6 & $6.315 \times 10^{-06}$ & $21 / 25$ & $2.1 \times 10^{+14}$ & 4.90 & 8.4 \\
\hline
\end{tabular}

with diverged solutions. All the trajectories producing rank deficiencies are ignored, because the $\kappa(\mathbf{J})$ for those trajectories are expected to be high. There are several straight line trajectories producing full rank $\mathbf{J}$, the condition numbers corresponding to those trajectories are found to be within the range of $10^{8} \leq \kappa(\mathbf{J}) \leq 10^{11}$ and the range of $10^{7} \leq \kappa(\mathbf{J}) \leq 10^{10}$ for $\mathrm{AMC}$ and $\mathrm{RMC}$, respectively. The identified solutions $\boldsymbol{\zeta}_{B}$ based on these condition numbers are not reliable enough. 
Table 4.10: The mean results for straight line trajectories.

\begin{tabular}{cc|cr|cc}
\hline & & \multicolumn{2}{|c}{ AMC } & \multicolumn{2}{c}{$\mathrm{RMC}$} \\
\hline $\mathrm{L}$ & $\mathrm{T}$ & $k_{\text {iter }}$ & $\operatorname{rank}(\mathbf{J})_{\text {avg }}$ & $k_{\text {iter }}$ & $\operatorname{rank}(\mathbf{J})_{\text {avg }}$ \\
\hline (a) & 1 & 29 & $18 / 28$ & 29 & $15 / 25$ \\
& 2 & 23 & $28 / 28$ & 27 & $25 / 25$ \\
& 3 & 29 & $18 / 28$ & 28 & $15 / 25$ \\
(b) & 1 & 7 & $28 / 28$ & 0 & - \\
& 2 & 27 & $27.96 / 28$ & 1 & $25 / 25$ \\
& 3 & 14 & $24 / 28$ & 0 & - \\
(c) & 1 & 29 & $18 / 28$ & 28 & $15 / 25$ \\
& 2 & 24 & $24 / 28$ & 0 & - \\
& 3 & 29 & $18 / 28$ & 0 & - \\
(d) & 1 & 30 & $27.97 / 28$ & 30 & $25 / 25$ \\
& 2 & 27 & $28 / 28$ & 21 & $25 / 25$ \\
& 3 & 27 & $24 / 28$ & 27 & $21 / 25$ \\
(e) & 1 & 29 & $18 / 28$ & 29 & $15 / 25$ \\
& 2 & 25 & $28 / 28$ & 22 & $25 / 25$ \\
& 3 & 29 & $18 / 28$ & 29 & $15 / 25$ \\
(f) & 1 & 28 & $28 / 28$ & 28 & $25 / 25$ \\
& 2 & 22 & $28 / 28$ & 17 & $25 / 25$ \\
& 3 & 28 & $24 / 28$ & 27 & $21 / 25$ \\
\hline \hline
\end{tabular}

Number of Simulations: 30

Number of Poses: 50

Table 4.10 summarizes the simulation results after performing 30 runs of both $\mathrm{AMC}$ and $\mathrm{RMC}$ for each straight line trajectory. The columns $k_{i t e r}$ represent the number of times that each trajectory produced a converged solution in the calibration. The columns $\operatorname{rank}(\mathbf{J})_{\text {avg }}$ are the average of $\operatorname{rank}(\mathbf{J})$ observed in the 30 runs.

Performing the measurement for kinematic calibration by commanding the manipulator to move its end-effector along a straight line trajectory may be simple, and the measurement system may also be easy to setup, but it was shown here that the identified solution $\boldsymbol{\zeta}_{B}$ are not reliable enough to correctly model $\boldsymbol{\zeta}_{A}$. 


\section{Planar Trajectories}

Three different planar trajectories of the manipulator end-effector positions, as presented in Figure 4.10, were analyzed. $\mathrm{P}=(\mathrm{a}),(\mathrm{b})$, and $(\mathrm{c})$ are the planes on the $\mathbf{y z}, \mathbf{x z}$, and $\mathbf{x y}$-planes, respectively. The end-effector is also oriented in three main directions, $\mathrm{T}=1,2,3$, just like the straight line trajectories analysed previously.

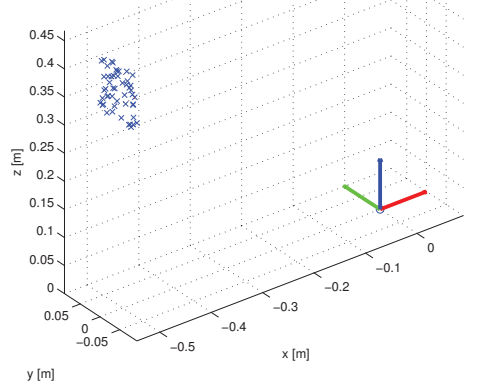

(a)

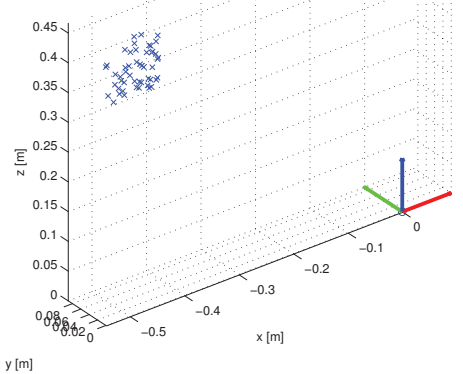

(b)

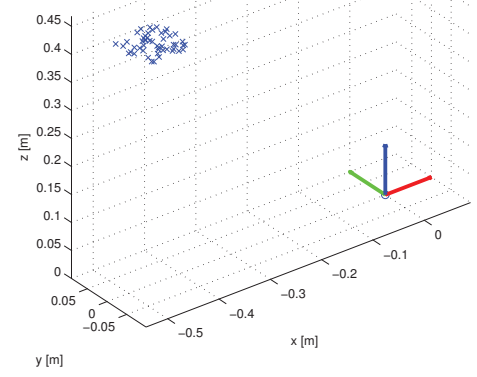

(c)

Figure 4.10: Three varieties of planar trajectories of end-effector pose. 
Table 4.11: Absolute measurement concept results using planar trajectories.

\begin{tabular}{|c|c|c|c|c|c|c|c|}
\hline \multicolumn{8}{|c|}{ Absolute Measurement Concept } \\
\hline $\mathrm{P}$ & $\mathrm{T}$ & $n_{\text {iter }}$ & res & $\operatorname{rank}(\mathbf{J})$ & $\kappa(\mathbf{J})$ & $\delta_{p}[\mu \mathrm{m}]$ & $\overline{\delta_{r}[\mu \mathrm{rad}]}$ \\
\hline \multirow[t]{3}{*}{ (a) } & 1 & 16 & $5.274 \times 10^{-06}$ & $28 / 28$ & $1.52 \times 10^{+06}$ & 0.01201 & 2.637 \\
\hline & 2 & 15 & $8.508 \times 10^{-06}$ & $28 / 28$ & $4.21 \times 10^{+06}$ & 1.129 & 4.101 \\
\hline & 3 & 16 & $5.742 \times 10^{-06}$ & $24 / 28$ & $2.19 \times 10^{+16}$ & 0.6639 & 2.793 \\
\hline \multirow[t]{3}{*}{ (b) } & 1 & 17 & $8.625 \times 10^{-06}$ & $18 / 28$ & $8.43 \times 10^{+16}$ & 0.5465 & 4.253 \\
\hline & 2 & 15 & $6.526 \times 10^{-06}$ & $28 / 28$ & $1.23 \times 10^{+07}$ & 2.565 & 2.017 \\
\hline & 3 & 17 & $9.484 \times 10^{-06}$ & $18 / 28$ & $6.60 \times 10^{+16}$ & 0.0378 & 4.731 \\
\hline \multirow[t]{3}{*}{ (c) } & 1 & 16 & $6.568 \times 10^{-06}$ & $28 / 28$ & $8.15 \times 10^{+05}$ & 0.3644 & 3.264 \\
\hline & 2 & 15 & $9.534 \times 10^{-06}$ & $28 / 28$ & $3.31 \times 10^{+06}$ & 0.6619 & 4.721 \\
\hline & 3 & 16 & $5.456 \times 10^{-06}$ & $24 / 28$ & $1.70 \times 10^{+16}$ & 0.7682 & 2.617 \\
\hline
\end{tabular}

Table 4.12: Relative measurement concept results using planar trajectories.

\begin{tabular}{cc|cccccc}
\hline \multicolumn{6}{c}{ Relative Measurement Concept } \\
\hline $\mathrm{P}$ & $\mathrm{T}$ & $n_{\text {iter }}$ & res & $\operatorname{rank}(\mathbf{J})$ & $\kappa(\mathbf{J})$ & $\delta_{p}\left[10^{3} \mu \mathrm{m}\right]$ & $\delta_{r}[\mu \mathrm{rad}]$ \\
\hline$(\mathrm{a})$ & 1 & 16 & $5.841 \times 10^{-06}$ & $25 / 25$ & $2.656 \times 10^{+04}$ & 20.64 & 10.41 \\
& 2 & 11 & $8.968 \times 10^{-06}$ & $25 / 25$ & $1.551 \times 10^{+04}$ & 20.65 & 665.8 \\
& 3 & 10 & $7.019 \times 10^{-06}$ & $21 / 25$ & $3.811 \times 10^{+15}$ & 20.64 & 1401 \\
$(\mathrm{~b})$ & 1 & 8 & $9.923 \times 10^{-06}$ & $15 / 25$ & $1.607 \times 10^{+20}$ & 67.87 & 3168 \\
& 2 & 10 & $8.609 \times 10^{-06}$ & $25 / 25$ & $1.168 \times 10^{+06}$ & 20.62 & 1121 \\
& 3 & 11 & $6.415 \times 10^{-06}$ & $15 / 25$ & $5.669 \times 10^{+20}$ & 67.9 & 394 \\
$(\mathrm{c})$ & 1 & 16 & $9.021 \times 10^{-06}$ & $25 / 25$ & $1.242 \times 10^{+05}$ & 20.64 & 11.05 \\
& 2 & 12 & $5.056 \times 10^{-06}$ & $25 / 25$ & $1.661 \times 10^{+04}$ & 20.65 & 349 \\
& 3 & 11 & $5.949 \times 10^{-06}$ & $21 / 25$ & $4.351 \times 10^{+15}$ & 20.64 & 681 \\
\hline \hline
\end{tabular}

Tables 4.11 and 4.12 summarize the first iteration of the planar trajectory simulation using AMC and RMC, respectively. There are five planar trajectories producing full rank $\mathbf{J}$, the condition numbers corresponding to those trajectories are within the range of $10^{5} \leq \kappa(\mathbf{J}) \leq 10^{8}$ and the range of $10^{4} \leq \kappa(\mathbf{J}) \leq 10^{7}$ for $\mathrm{AMC}$ and 
RMC, respectively. These condition numbers have improved by a factor of roughly $10^{3}$ compared to the results with straight line trajectories.

Table 4.13 summarizes the simulation results after performing 30 runs of both AMC and RMC for each planar trajectory. Every trajectory seemed to have almost a $100 \%$ convergence, and many of them resulted in a full rank $\mathbf{J}$. The planar trajectories that produced full rank have better results than the ones in straight line trajectories, but it is still impractical for planar trajectories to be used for kinematic calibration with either the AMC or RMC with the $\kappa(\mathbf{J})$ this high.

Table 4.13: The mean results for planar trajectories.

\begin{tabular}{cc|cc|cc}
\hline & & \multicolumn{2}{|c}{ AMC } & \multicolumn{2}{c}{ RMC } \\
\hline $\mathrm{P}$ & $\mathrm{T}$ & $k_{\text {iter }}$ & $\operatorname{rank}(\mathbf{J})_{\text {avg }}$ & $k_{\text {iter }}$ & $\operatorname{rank}(\mathbf{J})_{\text {avg }}$ \\
\hline (a) & 1 & 29 & $28 / 28$ & 30 & $25 / 25$ \\
& 2 & 30 & $28 / 28$ & 30 & $25 / 25$ \\
& 3 & 28 & $24 / 28$ & 30 & $21 / 25$ \\
(b) & 1 & 30 & $18 / 28$ & 30 & $15 / 25$ \\
& 2 & 30 & $28 / 28$ & 30 & $25 / 25$ \\
& 3 & 30 & $18 / 28$ & 30 & $15 / 25$ \\
(c) & 1 & 30 & $28 / 28$ & 30 & $25 / 25$ \\
& 2 & 30 & $28 / 28$ & 30 & $25 / 25$ \\
& 3 & 30 & $24 / 28$ & 30 & $21 / 25$ \\
\hline \hline
\end{tabular}

Number of Simulations: 30

Number of Poses: 50

\section{Volumetric Trajectories}

Four different volumetric trajectories of the manipulator end-effector positions, as presented in Figure 4.11, were analysed. The first three trajectories $V=(\mathrm{a}),(\mathrm{b})$, and (c), are specified by extruding the planar trajectories $\mathrm{P}=(\mathrm{a}),(\mathrm{b}),(\mathrm{c})$, along the axis normal to the respective planes. The trajectory $\mathrm{V}=(\mathrm{d})$ is specified to be the same as the simulations in Section 4.2, which is to randomly select the joint angles of the 


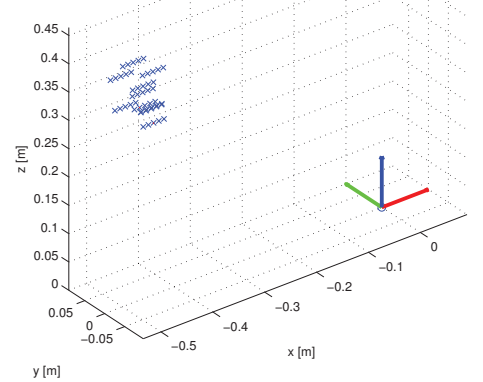

(a)

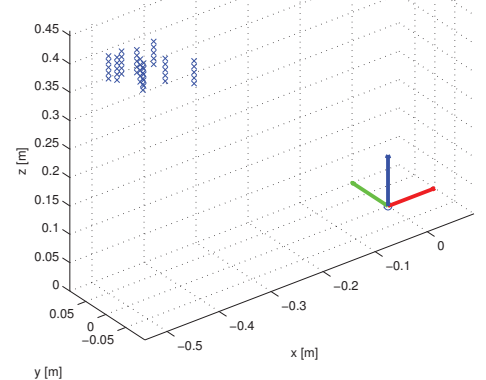

(c)

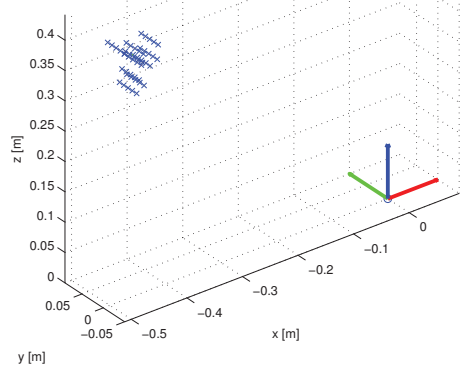

(b)

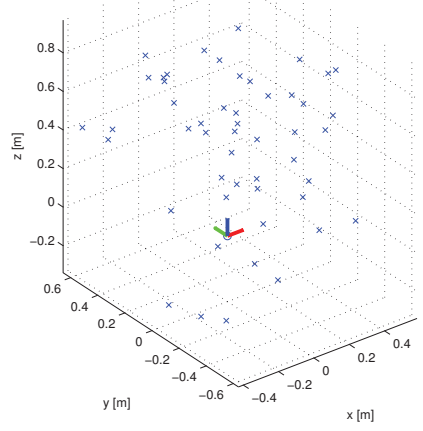

(d)

Figure 4.11: Four varieties of volumetric trajectories of end-effector pose.

respective joints within their joint limits, hence the inverse kinematic is not needed.

Tables 4.14 and 4.15 summarize the first iteration of the volumetric trajectories simulation using AMC and RMC, respectively. Seven of these volumetric trajectories produced full rank $\mathbf{J}$, the condition numbers corresponding to the trajectories are found to be in the range of $10^{3} \leq \kappa(\mathbf{J}) \leq 10^{7}$ and $10^{2} \leq \kappa(\mathbf{J}) \leq 10^{5}$ for AMC and RMC, respectively. The best performing $\kappa(\mathbf{J})$ is the trajectory $\mathrm{V}=(\mathrm{d})$, because the trajectory of the entire workspace is used for calibration. 
Table 4.14: Absolute measurement concept results using volumetric trajectories.

\begin{tabular}{cc|cccccc}
\hline \multicolumn{6}{c}{ Absolute Measurement Concept } \\
\hline $\mathrm{V}$ & $\mathrm{T}$ & $n_{\text {iter }}$ & res & $\operatorname{rank}(\mathbf{J})$ & $\kappa(\mathbf{J})$ & $\delta_{p}[\mu \mathrm{m}]$ & $\delta_{r}[\mu \mathrm{rad}]$ \\
\hline (a) & 1 & 14 & $6.343 \times 10^{-06}$ & $28 / 28$ & $3.117 \times 10^{+04}$ & 0.8344 & 3.06 \\
& 2 & 17 & $6.528 \times 10^{-06}$ & $28 / 28$ & $9.544 \times 10^{+05}$ & 0.3726 & 3.243 \\
& 3 & 17 & $6.723 \times 10^{-06}$ & $24 / 28$ & $2.064 \times 10^{+16}$ & 0.3567 & 3.341 \\
(b) & 1 & 14 & $8.912 \times 10^{-06}$ & $28 / 28$ & $2.836 \times 10^{+04}$ & 2849 & 3.425 \\
& 2 & 17 & $6.638 \times 10^{-06}$ & $28 / 28$ & $1.245 \times 10^{+06}$ & 0.3225 & 3.303 \\
& 3 & 17 & $6.684 \times 10^{-06}$ & $24 / 28$ & $2.764 \times 10^{+16}$ & 0.4073 & 3.315 \\
$(\mathrm{c})$ & 1 & 16 & $5.494 \times 10^{-06}$ & $28 / 28$ & $2.333 \times 10^{+04}$ & 0.3315 & 2.727 \\
& 2 & 17 & $6.657 \times 10^{-06}$ & $28 / 28$ & $8.101 \times 10^{+05}$ & 0.2682 & 3.318 \\
& 3 & 17 & $6.703 \times 10^{-06}$ & $24 / 28$ & $1.987 \times 10^{+16}$ & 0.3364 & 3.334 \\
$(\mathrm{~d})$ & - & 17 & $6.725 \times 10^{-06}$ & $28 / 28$ & 1171 & 0.249 & 3.353 \\
\hline \hline
\end{tabular}

Table 4.15: Relative measurement concept results using volumetric trajectories.

\begin{tabular}{cc|cccccc}
\hline \multicolumn{6}{c}{ Relative Measurement Concept } \\
\hline $\mathrm{V}$ & $\mathrm{T}$ & $n_{\text {iter }}$ & res & $\operatorname{rank}(\mathbf{J})$ & $\kappa(\mathbf{J})$ & $\delta_{p}\left[10^{3} \mu \mathrm{m}\right]$ & $\delta_{r}[\mu \mathrm{rad}]$ \\
\hline$(\mathrm{a})$ & 1 & 15 & $8.941 \times 10^{-06}$ & $25 / 25$ & $1.734 \times 10^{+04}$ & 37.79 & 0.1292 \\
& 2 & 11 & $7.814 \times 10^{-06}$ & $25 / 25$ & 7361 & 37.78 & 41.23 \\
& 3 & 11 & $9.402 \times 10^{-06}$ & $21 / 25$ & $4.478 \times 10^{+15}$ & 37.77 & 43.82 \\
$(\mathrm{~b})$ & 1 & 12 & $6.856 \times 10^{-06}$ & $25 / 25$ & 7929 & 37.79 & 10.92 \\
& 2 & 9 & $7.405 \times 10^{-06}$ & $25 / 25$ & 7419 & 37.71 & 159.5 \\
& 3 & 10 & $8.704 \times 10^{-06}$ & $21 / 25$ & $4.375 \times 10^{+15}$ & 37.77 & 83.67 \\
$(\mathrm{c})$ & 1 & 16 & $5.393 \times 10^{-06}$ & $25 / 25$ & $1.808 \times 10^{+04}$ & 37.79 & 6223 \\
& 2 & 11 & $9.389 \times 10^{-06}$ & $25 / 25$ & 7339 & 37.76 & 38.42 \\
& 3 & 11 & $6.831 \times 10^{-06}$ & $21 / 25$ & $4.644 \times 10^{+15}$ & 37.77 & 43.02 \\
$(\mathrm{~d})$ & - & 17 & $9.252 \times 10^{-06}$ & $25 / 25$ & 725.9 & 37.79 & 3.231 \\
\hline \hline
\end{tabular}

Table 4.16 summarizes the simulation results after performing 30 runs of both AMC and RMC for each volumetric pose trajectory. Each pose trajectory appeared 
to have $100 \%$ convergence, and the majority of them caused no rank deficiencies. Considering $k_{i t e r}$, the averaged $\operatorname{rank}(\mathbf{J})$, and $\kappa(\mathbf{J}), \mathrm{V}=(\mathrm{d})$ produced the best results for kinematic calibration whether AMC or RMC were used. However, the measurement system would be hard to setup for this trajectory.

Table 4.16: The mean results for volumetric trajectories.

\begin{tabular}{cc|cc|cc}
\hline & & \multicolumn{2}{|c}{ AMC } & \multicolumn{2}{c}{ RMC } \\
\hline $\mathrm{V}$ & $\mathrm{T}$ & $k_{\text {iter }}$ & $\operatorname{rank}(\mathbf{J})$ & $k_{\text {iter }}$ & $\operatorname{rank}(\mathbf{J})$ \\
\hline (a) & 1 & 30 & $28 / 28$ & 30 & $25 / 25$ \\
& 2 & 30 & $28 / 28$ & 30 & $25 / 25$ \\
& 3 & 30 & $24 / 28$ & 30 & $21 / 25$ \\
(b) & 1 & 30 & $28 / 28$ & 30 & $25 / 25$ \\
& 2 & 30 & $28 / 28$ & 30 & $25 / 25$ \\
& 3 & 30 & $24 / 28$ & 30 & $21 / 25$ \\
(c) & 1 & 30 & $28 / 28$ & 30 & $25 / 25$ \\
& 2 & 30 & $28 / 28$ & 30 & $25 / 25$ \\
& 3 & 30 & $24 / 28$ & 30 & $21 / 25$ \\
(d) & - & 30 & $28 / 28$ & 30 & $25 / 25$ \\
\hline \multicolumn{6}{r}{ Number of Simulations: 30} \\
\multicolumn{6}{l}{ Number of Poses: 50}
\end{tabular}

In this thesis, an experiment was setup for using the Thermo CRS A465 with its end-effector moving in a planar trajectory always pointing down. Another experiment was conducted using the 7 DOF WAM Arm with the end-effector moving in the trajectory generated by specifying random joint angles within their respective joint limits. Both experimental setups and the results are discussed in the next chapter. 


\section{Chapter 5}

\section{Experimental Setup and Results}

\subsection{End-Effector Pose Measurements}

In this chapter, the pose measurements for the kinematic calibration are performed using computer vision. In computer vision applications, the full pose information can be determined from camera calibration, or stereo vision [24]. A camera calibration algorithm is used to obtain the full pose information for kinematic calibration, the results are reported in this chapter.

Camera calibration implies the computation of intrinsic and extrinsic parameters that define the camera model. The intrinsic parameters map the points describing the target object in 3D Euclidean space (viewed by the camera) and the corresponding

points projected to the image plane. The extrinsic parameters describe the pose of the camera relative to the target object. In order to obtain precise estimates of the parameters, the camera model must account for the effects of lens distortion as well. By attaching either a target or a camera to the end-effector, the end-effector pose can be extracted from the extrinsic parameters [21-23]. 


\subsubsection{Camera Calibration}

The goal for the camera calibration is to estimate the intrinsic and extrinsic parameters, and the lens distortion coefficient. Like kinematic calibration, the camera calibration process is also a form of nonlinear optimization.

\section{Calibration Board}

A target with a known geometry is needed for camera calibration, the identifiable points on the target can be represented in the physical world (Euclidean-3 space $E_{3}$ ) as the homogeneous coordinates, $\mathbf{Q}=(X, Y, Z, 1)$. An image taken of the target with the camera consists of the same identifiable points in the image space (Projective-2 space $P_{2}$ ), which can also be represented in homogeneous coordinates, $\mathbf{q}=(x, y, 1)$.

By using a planar rectangular pattern as the target, there are four distinctive identifiable points in $E_{2}$ (because the pattern is planar, hence $\mathrm{Z}=0$ ), and they are $\left(X_{1}, Y_{1}, 1\right),\left(X_{2}, Y_{2}, 1\right),\left(X_{3}, Y_{3}, 1\right)$, and $\left(X_{4}, Y_{4}, 1\right)$. The corresponding points in $P_{2}$ are $\left(x_{1}, y_{1}, 1\right),\left(x_{2}, y_{2}, 1\right),\left(x_{3}, y_{3}, 1\right)$, and $\left(x_{4}, y_{4}, 1\right)$. Therefore, there are eight equations produced per image, since each point can be described by two equations.

There are four intrinsic parameters $\left(f_{x}, f_{y}, c_{x}, c_{y}\right)$, six extrinsic parameters $(3$ for position and 3 for orientation), and five distortion coefficients $\left(k_{1}, k_{2}, k_{3}\right.$ for radial and $p_{1}, p_{2}$ for tangential) that need to be estimated in the camera calibration process. The four intrinsic parameters are properties of the camera, these parameters do not change between the views. Each image of the rectangular pattern produces eight equations at the cost of introducing six extrinsic parameters. This means that at least two distinctive images (introduce 16 equations) must be taken to solve for the four intrinsic parameters (16 parameters in total, where there are four intrinsic and 12 extrinsic parameters). 
The camera lens distorts the object in the image space, which means that straight lines become curved in the image. However, a rectangular pattern formed by only four identifiable points does not include the information on the curvature between points. Therefore, a greater number of identifiable points (internal corners) are needed. Since there are five distortion coefficients to be identified, implying that five equations are needed. Because a planar pattern with three internal corners yields six equations (recall that each point yields two equations), thus only one image of the planar pattern with at least three internal corners is needed to solve for all five coefficients.

All of this implies that at least two perspective views of a $3 \times 2$ patterned calibration board are the minimum requirement for solving the camera calibration problem. However, with the consideration for noises and numerical stability, it typically requires more views of the larger patterned calibration board. According to [52], for high quality results in practice, at least ten views of a $7 \times 8$ or larger pattern is needed. The even-odd symmetry pattern on the calibration board has only one symmetrical axis, allowing the orientation of the calibration board to be determined uniquely. Based on all the requirements above, the calibration board used for this thesis has a $7 \times 10$ pattern, as shown in Figure 5.1.

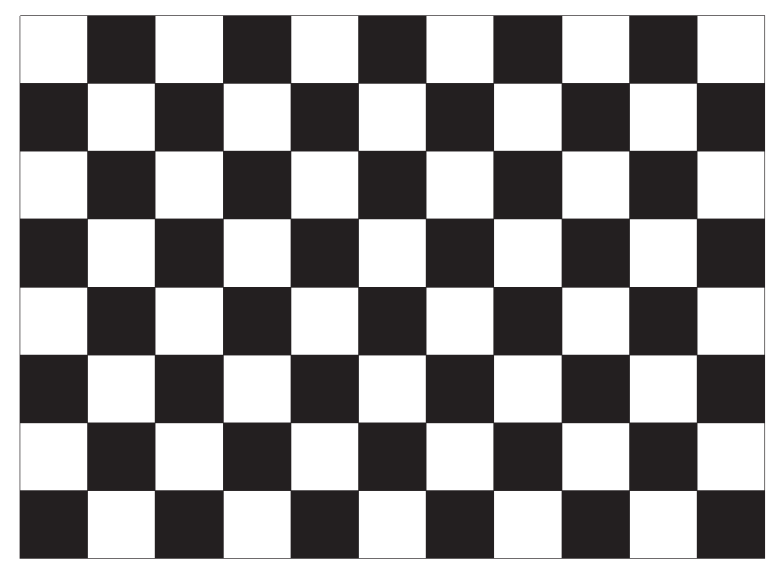

Figure 5.1: Illustration of the $7 \times 10$ camera calibration board. 


\section{Camera Calibration Process}

Because the camera calibration is also a form of nonlinear optimization, the parameters are identified iteratively. However, the accuracy in the solution of the intrinsic and extrinsic parameters are highly dependent on the distortion coefficients. Therefore, all 14 parameters in the camera calibration cannot be solved at the same time like the process for kinematic calibration.

Once an image of the calibration board is taken, the corresponding first point is found in $P_{2}$ and the remaining points are defined relative to this point. Since the identifiable points in both $E_{3}$ and $P_{2}$ are expressed in terms of homogeneous coordinates, the homography matrix $\mathbf{H}$ can be determined [52]. The homography matrix $\mathbf{H}$ takes the form of

$$
\mathbf{H}=\left[\begin{array}{ccc}
f_{x} & 0 & c_{x} \\
0 & f_{y} & c_{y} \\
0 & 0 & 1
\end{array}\right]\left[\begin{array}{ll}
\mathbf{R} & \mathbf{p}
\end{array}\right]
$$

where $f_{x}, f_{y}, c_{x}$, and $c_{y}$ are the intrinsic parameters, $\mathbf{R}$ is the $3 \times 3$ orthogonal matrix representing the orientation of the camera relative to the target, and $\mathbf{p}$ is the $3 \times 1$ vector representing the position of the camera relative to the target.

Each image taken by the camera is distorted both radially and tangentially. These effects are characterized by the distortion coefficients defined using the equations

$$
\left[\begin{array}{l}
x_{c} \\
y_{c}
\end{array}\right]=\left(1+k_{1} r^{2}+k_{2} r^{4}+k_{3} r^{6}\right)\left[\begin{array}{l}
x_{d} \\
y_{d}
\end{array}\right]+\left[\begin{array}{c}
2 p_{1} x_{d} y_{d}+p_{2}\left(r^{2}+2 x_{d}^{2}\right) \\
p_{1}\left(r^{2}+2 y_{d}^{2}\right)+2 p_{2} x_{d} y_{d}
\end{array}\right],
$$


where $\left(x_{d}, y_{d}\right)$ is the location of each identifiable point in the raw image. The location of the corresponding identifiable points after being corrected is $\left(x_{c}, y_{c}\right)$, and $r^{2}=$ $x_{d}^{2}+y_{d}^{2}$. A large set of these equations are collected and solved to identify distortion coefficients. Each of these equations correspond to the $m \times n$ internal corners on the calibration board, resulting in $m n$ equations.

Once the corrected identifiable points $\left(x_{c}, y_{c}\right)$ are obtained from Eq. (5.2), the location of the points are compared to the actual known $(x, y)$ location of the corre-

sponding points on the target. If the norm of the errors between $\left(x_{c}, y_{c}\right)$ and $(x, y)$ is not satisfactory, the algorithm determines $\mathbf{H}$ again using the new corrected points $\left(x_{c}, y_{c}\right)$.

\subsubsection{Camera Calibration Results}

As previously mentioned, during the camera calibration process, multiple perspective views of the calibration board are needed to yield useful results. The process used here is illustrated in Figure 5.2, the calibration board used has a pattern size of $7 \times 10$ internal corners, and 14 different perspective views were used.

Three types of digital cameras were used to develop the camera calibration algorithm. The first one is a simple low cost camera that is referred to as "Cam". The images taken by this camera have a resolution of $640 \times 480$. The second one is the webcam embedded on the 13 in. MacBook Pro, which is called "iSight" by Apple Inc.. This camera is capable of taking images with a resolution of $1280 \times 720$. The third one is a HD webcam by Creative Technology, Ltd.. This camera is capable of video capturing resolution of HD 720p with a frame rate of $30 \mathrm{fps}$. The images taken from this camera have a resolution of $1280 \times 720$. The calibration results of the three types of cameras are summarized in Table 5.1. 


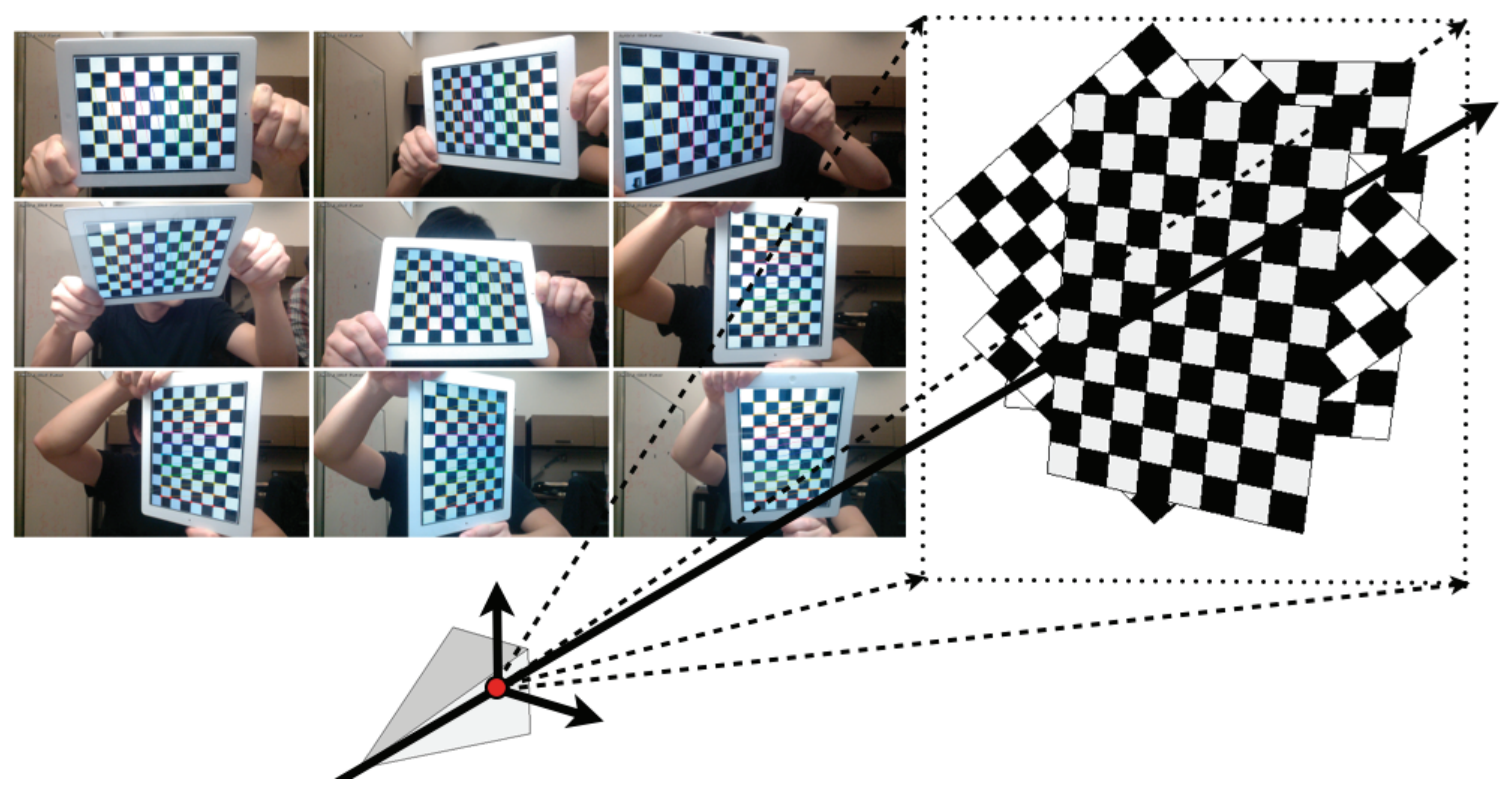

Figure 5.2: Illustration of the camera calibration process.

Given the resolutions of each camera, the identified principle axes locations $\left(c_{x}\right.$, $\left.c_{y}\right)$ are located approximately in the centre of the images taken from each camera. It is shown that the focal length $\left(f_{x}, f_{y}\right)$ of the iSight is the shortest, which is reasonable because iSight is an embedded laptop camera and the other two are standard CCD

Table 5.1: Camera calibration results of the camera internal properties.

\begin{tabular}{|c|c|c|c|}
\hline Paramete & Cam & iSight & Creative \\
\hline$k_{1}$ & -0.3482 & -0.1960 & -0.1717 \\
\hline$k_{2}$ & 4.3605 & 1.0325 & 2.7260 \\
\hline$k_{3}$ & 0.0006 & 0.0032 & -0.0020 \\
\hline$p_{1}$ & -0.0004 & 0.0043 & 0.0009 \\
\hline$p_{2}$ & -48.357 & -2.5930 & -14.099 \\
\hline$[\mathrm{px}]$. & 1224.2 & 993.59 & 1220.5 \\
\hline [px.] & 1224.2 & 993.80 & 1219.7 \\
\hline$[\mathrm{px}]$. & 342.23 & 626.69 & 656.33 \\
\hline [px.] & 252.70 & 337.84 & 379.17 \\
\hline RMS [px.] & 0.6863 & 0.2137 & 0.2287 \\
\hline
\end{tabular}


cameras. The RMS value in the table represents the error in the corrected location of the identifiable points to the corresponding image pixels.

\subsection{Experiment using the Thermo CRS A465}

The first experiment described here uses the RMC on the Thermo CRS A465. "Cam" was attached to the end-effector targeting the calibration board, as illustrated in Figure 5.3. The actual end-effector pose can be extracted from the extrinsic parameters of "Cam", by applying the DH transformation of $\Sigma_{6} \rightarrow \Sigma_{n+1}$.

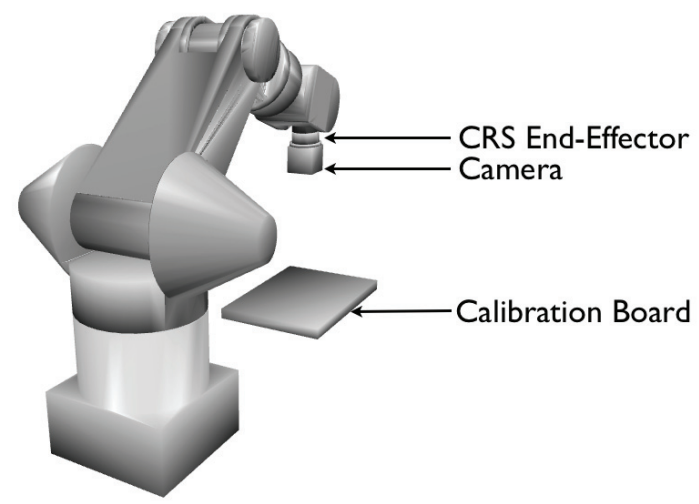

Figure 5.3: Relative measurement setup for a Thermo CRS A465.

The Thermo CRS A465 was mounted to an aluminum surface of 1,524 $\mathrm{mm} \times$ $1,219 \mathrm{~mm} \times 12.7 \mathrm{~mm}$, that was rigidly fastened to a steel table, just like the setup in [4]. The A465 controller is a C500C, which is connected to a 13 in. MacBook Pro using a Serial-USB converter.

\subsubsection{Thermo CRS A465 Programming}

The C500C controller uses the robot operating system developed by Thermo Inc. called CROS. CROS can only interpret the RAPL-3 programming language. 
Robcomm3 is a Windows-based software designed for use with the RAPL-3 programming language. It is an integrated development environment (IDE) that allows users to create programs/applications for the robot, or communicate interactively with the controller using a terminal window. However, Robcomm3 does not work in a Mac OS X operating system platform, instead pyserial is used to access the serial communication with the controller using the Mac terminal.

A number of functions were written in Python for controlling the A465 using the pyserial module. The A465 end-effector is commanded to move in a world coordinate trajectory using the function, CRSworld (a Python function that was written to move the A465), continuously. The arguments used by CRSworld are the coordinate axis to move along and the displacements in $\mathrm{mm}$. The joint angles that establish each configuration are then recorded using readCRS (another function that was written for the A465), which allows the user to record outputs from in the C500C controller: either the joint angles measured by the joint transducers, or the end-effector pose with respect to the world coordinate frame.

\subsubsection{End-Effector Pose Measurements}

In this experiment, the Thermo CRS A465 is commanded to move along a certain trajectory on the $\mathbf{x y}$-plane of the robot base frame. Using the joint angles recorded the forward kinematics are computed, resulting in the trajectory shown in Figure 5.4(a). At each commanded pose, an image of the calibration board is taken, and the extrinsic parameters of the camera are extracted, revealing the trajectory in Figure 5.4(b). It is important to note that the former is computed relative to $\Sigma_{b}$, and the latter is the camera pose relative to $\Sigma_{m}$. 


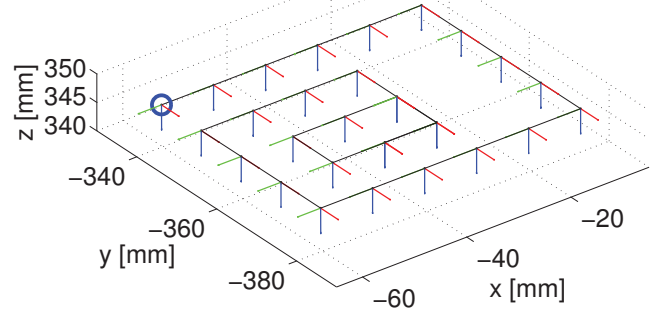

(a)

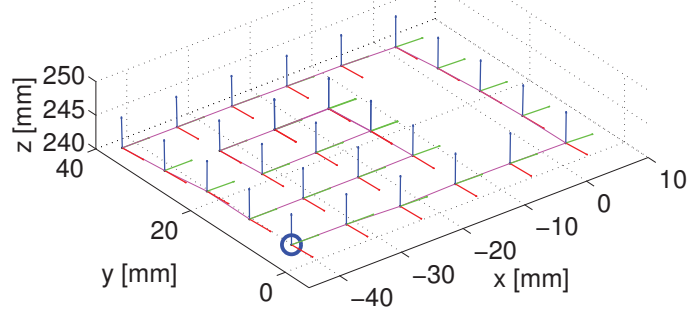

(b)

Figure 5.4: (a) The computed A465 poses using the recorded joint angles, giving the nominal model. (b) The extrinsic parameters obtained using OpenCV, giving the measured model.

In order to correctly use the relative errors in Eq. (2.6), the reference frames used to express the poses in Figure 5.4 must have the same orientation. Using the transformation to orient the frames and Eq. (2.6), the poses expressed relative to the location $(0,0,0)$ are illustrated in Figure 5.5. The errors between the nominal and actual poses observed in Figure 5.5 are the relative errors.

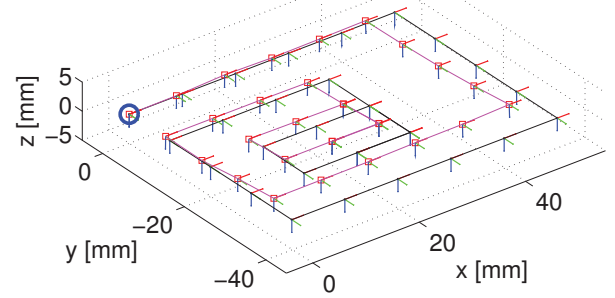

Figure 5.5: The relative pose of the nominal and measured models. 


\subsubsection{A465 Kinematic Calibration Results}

Using the relative errors in Figure 5.5, the A465 can be calibrated using RMC, and the results are summarized in Table 5.2. The results were obtained after 100 iterations, but the numerical residual of the calibration algorithm diverged. The implication is that the RMC was not useful in this experiment. Another implication is that the planar pose trajectory chosen was not suitable for this experiment.

Table 5.2: Experimental results for the relative measurement concept.

\begin{tabular}{crrrr}
\hline & \multicolumn{2}{c}{ Joint Errors $[\mathrm{deg}]$} & \multicolumn{2}{c}{ Link Errors $[\mathrm{mm}]$} \\
\hline \multicolumn{1}{c}{$\delta \boldsymbol{\theta}_{i d}$} & $\delta \boldsymbol{\alpha}_{i d}$ & $\delta \boldsymbol{a}_{i d}$ & $\delta \boldsymbol{d}_{i d}$ \\
\hline$b$ & -398.6969 & 10.3722 & 0.0000 & 0.0000 \\
1 & $-2,113.9504$ & 594.4484 & 875.7930 & 0.0000 \\
2 & -122.4237 & -255.8268 & $-1,872.5064$ & $-5,523.0542$ \\
3 & $-3,161.6930$ & 399.8710 & $8,489.8435$ & 0.0000 \\
4 & $12,422.4892$ & 89.9887 & -607.1022 & $-5,983,959.6130$ \\
5 & $-11,465.0984$ & -397.8224 & $-2,164.5666$ & $6,032,473.7731$ \\
6 & $1,292.1277$ & $-1,747.9447$ & -341.3226 & -167.7872 \\
$n+1$ & -894.5268 & 0.0000 & 0.0000 & 64.8227 \\
\hline 3 & $\delta \boldsymbol{\beta}_{i d}$ & -5125.346 & &
\end{tabular}

A similar planar pose trajectory was analyzed in Section 4.3.4, which resulted in a high condition number, $\kappa(\mathbf{J})$. This implies that the identified kinematic parameters are not reliable enough, leading to the conclusion that the planar trajectory is not ideal for kinematic calibration. Based on the simulations in Section 4.3.4, the best kinematic calibration result is obtained when the end-effector is moved in a volumetric trajectory with large moving space. The next section reports the experiment of the kinematic calibration using the WAM Arm when it is moved in a volumetric trajectory. 


\subsection{Experiment using WAM Arm}

The calibration board was attached to the end-effector and the camera faced towards the robot instead, as illustrated in Figure 5.6. The WAM Arm is capable of reading the absolute joint angles to configure the robot instead of moving each joint relative to the previous configuration like the A465. This means that the configuration of the arm can be specified using the generated joint angles used in the simulations.

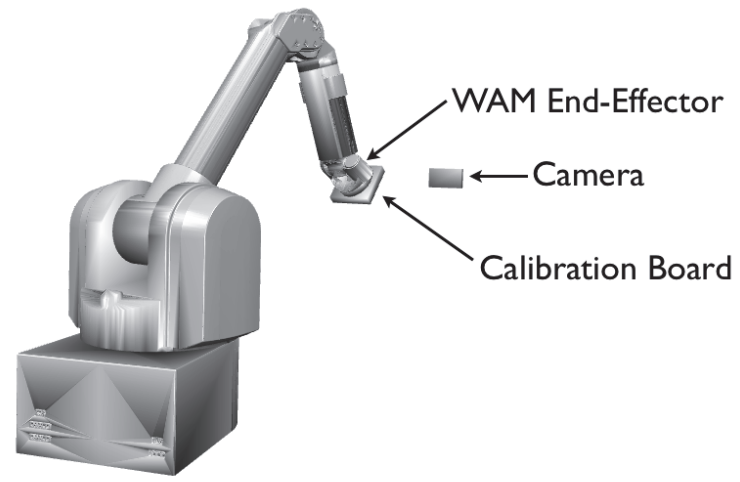

Figure 5.6: Relative measurement setup for a WAM Arm.

\subsubsection{Generating Pose Trajectory}

A simulation was performed to generate joint angles suitable for kinematic calibration. In this simulation, a camera is placed in the $E_{3}$ space facing towards the WAM, then the WAM had its end-effector facing towards the camera using the property of the spherical wrist while the remaining joint angles varied randomly within their joint limits. Only a subset of the simulated configurations were chosen for the experiments. 


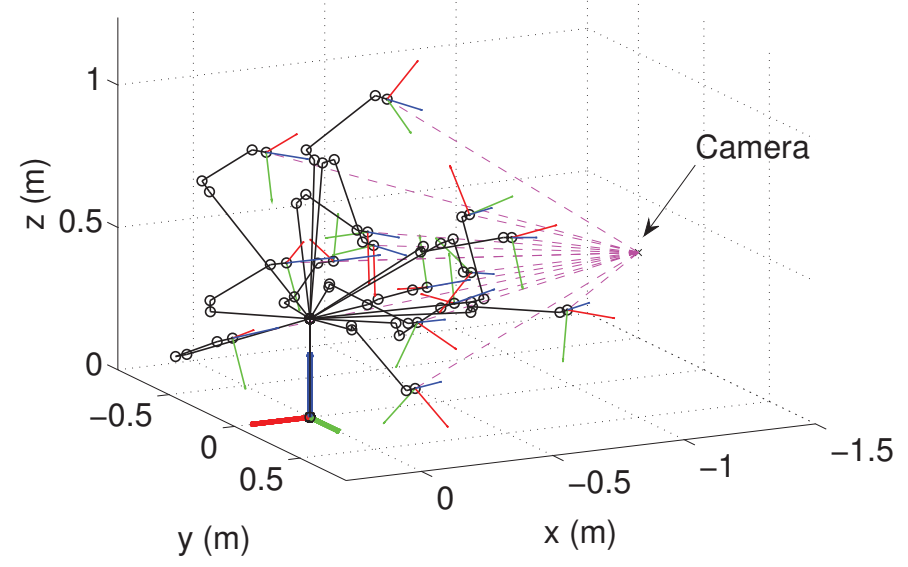

Figure 5.7: The kinematic model of the WAM in simulation.

Figure 5.7 shows the kinematic model of the 7 DOF WAM Arm with varying joint angles. In this figure, only the subset of the simulated kinematic model is presented, meaning that only the first 15 configurations of the total number of configurations are illustrated. In this simulation, the manipulator base is placed at the Cartesian coordinates $(0,0,0)$, and the location of the camera is placed relative to the base at $(-1.5,0.5,0.3)$, the units are in $\mathrm{m}$.

\section{Maintaining the End-Effector Orientation to Track the Camera}

By using the property of the spherical wrist configuration in the WAM Arm, the end-effector can be moved to any point in $E_{3}$ with many possible orientations. Using the end-effector pose for extracting the required joint configurations is an inverse kinematics problem, but the inverse kinematics problem for a 7 DOF manipulator can be extremely complicated due to the kinematic redundancy. Fortunately, it is not required to perform a complete inverse kinematics analysis for the 7 DOF serial manipulator here, the inverse kinematics is only applied for the spherical wrist in order to maintain the orientation of the end-effector when it is randomly positioned. 
In order to keep track of the end-effector orientation about the camera, the position of the end-effector relative to the camera must be known (implying that the location of the camera must be known in $E_{3}$ ). Because the $\mathbf{x}, \mathbf{y}, \mathbf{z}$-axes of the end-effector are defined to be orthonormal vectors. First, define the $\mathbf{z}$-axis of the end-effector to be the unit vector $\overrightarrow{\mathbf{a}}_{e}$,

$$
\overrightarrow{\mathbf{a}}_{e}=\frac{\mathbf{p}_{c a m}-\mathbf{p}_{e}}{\left\|\mathbf{p}_{c a m}-\mathbf{p}_{e}\right\|_{2}}
$$

where $\mathbf{p}_{c a m}$ and $\mathbf{p}_{e}$ are the location of the camera and the location of the end-effector, respectively, and they are both expressed relative to the world coordinate frame. Eq. (5.3) allows the $\mathbf{z}$-axis on the end-effector to constantly point at the camera.

The remaining two axes can be defined in an infinite number of possible ways, because the only interest here was just established, but they must still retain the orthonormal properties. The $\mathbf{x}$-axis with the unit vector $\overrightarrow{\mathbf{n}}_{e}$ is chosen as the randomly selected $3 \times 1$ vector consisting of the properties, $\left\|\overrightarrow{\mathbf{n}}_{e}\right\|_{2}=1$ and $\overrightarrow{\mathbf{n}}_{e} \cdot \overrightarrow{\mathbf{a}}_{e}=0$. Finally, the $\mathbf{y}$-axis with the unit vector $\overrightarrow{\mathbf{s}}_{e}$ is established by completing the right hand rule with the property of $\overrightarrow{\mathbf{s}}_{e}=\overrightarrow{\mathbf{a}}_{e} \times \overrightarrow{\mathbf{n}}_{e}$. The $\overrightarrow{\mathbf{n}}_{e}, \overrightarrow{\mathbf{s}}_{e}$, and $\overrightarrow{\mathbf{a}}_{e}$ unit vectors are stacked to get the rotational matrix of the end-effector ${ }^{b} \mathbf{R}_{e}$, which are represented relative to the manipulator base.

The inverse kinematics solution of a spherical wrist is derived in [48], only the orientation component ${ }^{w} \mathbf{R}_{e}$ is of interest here, which can be derived from ${ }^{b} \mathbf{R}_{e}$. The spherical wrist is configured using the final three joints $\left(\theta_{5}, \theta_{6}, \theta_{7}\right)$ of the $\mathrm{WAM}^{T M}$ Arm, meaning that ${ }^{w} \mathbf{R}_{e}$ can be derived from ${ }^{b} \mathbf{R}_{e}$ provided that the first four joints $\left(\theta_{1}, \theta_{2}, \theta_{3}\right.$, and $\left.\theta_{4}\right)$ are known. In this simulation, the first four joints are defined as randomly generated numbers within the joint limits, which produces ${ }^{b} \mathbf{R}_{\text {wrist }}$ and ${ }^{b} \mathbf{p}_{\text {wrist }}$. Therefore, the orientation of the spherical wrist is

$$
{ }^{\text {wrist }} \mathbf{R}_{e}={ }^{b} \mathbf{R}_{\text {wrist }}^{T}{ }^{b} \mathbf{R}_{e}
$$


Finally, the joint angles for maintaining the end-effector orientation to track the camera are extracted using the derived equations in [48]. There are two possible solutions,

$$
\begin{aligned}
& \theta_{4}=\arctan 2\left(a_{e, y}, a_{e, x}\right), \\
& \theta_{5}=\arctan 2\left(\sqrt{a_{e, x}^{2}+a_{e, y}^{2}}, a_{e, z}\right), \\
& \theta_{6}=\arctan 2\left(s_{e, z},-n_{e, z}\right),
\end{aligned}
$$

for $0 \leq \theta_{5} \leq \pi$, and

$$
\begin{aligned}
& \theta_{4}=\arctan 2\left(a_{e, y}, a_{e, x}\right), \\
& \theta_{5}=\arctan 2\left(-\sqrt{a_{e, x}^{2}+a_{e, y}^{2}}, a_{e, z}\right), \\
& \theta_{6}=\arctan 2\left(-s_{e, z}, n_{e, z}\right),
\end{aligned}
$$

for $0>\theta_{5} \geq-\pi$. Note that $s_{e, x}, s_{e, y}, s_{e, z}, n_{e, x}, n_{e, y}$, and $n_{e, z}$ are the $\mathbf{x}, \mathbf{y} \mathbf{z}$ components of the unit vectors, $\overrightarrow{\mathbf{s}}_{e}$ and $\overrightarrow{\mathbf{n}}_{e}$, respectively.

\section{Selecting Feasible Joint Angles}

Using the inverse kinematics solutions from Eqs. (5.5) and (5.6) can produce joint angles outside of the joint limits, and the randomly generated joint angles being within the respective joint limits of the first four joints can possibly cause the links to collide with surrounding objects. A fast and easy method was implemented here to overcome these problems for the joint angles by defining boundaries for the solution and removing the joint angles outside of these boundaries from the trajectory.

The joint angles used in the experiment were extracted from the kinematic model in the simulation. Many of the joint angles obtained were acceptable in the way that 
they were well within the joint limits and the task space of the experiment. However, there are a handfull of joint angles within the ones obtained from the simulation not working when they were implemented into the WAM, the images taken in some of the configurations were too dark for obtaining good camera calibration results. Therefore, those joint angles were also removed from the trajectory for the kinematic calibration experiment.

The simulation was set to generate 100 joint angles, 75 of them were selected to be feasible to feed into the WAM, but the camera calibration algorithm failed on 20 images. Therefore, there are 55 joint angles used for the experiment. According to Figure 4.8, 55 joint angles are more than enough to overcome the presence of measurement noise, and the number of poses produced are substantially more than the ones used in the previous experiment.

\subsubsection{Kinematic Calibration Results of the WAM}

Figure 5.8 presents the kinematic model of the 7 DOF WAM Arm using the joint angles selected from the simulation as discussed in Section 5.3.1. Only the first 15 poses of the kinematic model are shown.

Using the measured poses obtained from OpenCV, the absolute and relative accuracies before the WAM Arm is calibrated are presented in Figures 5.9(a) and 5.9(b). The absolute accuracies here are considerably lower than the ones presented in Figure 4.7(a), because the manipulator base placement and orientation are exaggerated a lot in the simulations performed earlier. The first 15 absolute and relative poses before calibration are presented in Figures 5.9(c) and 5.9(d), repectively. 


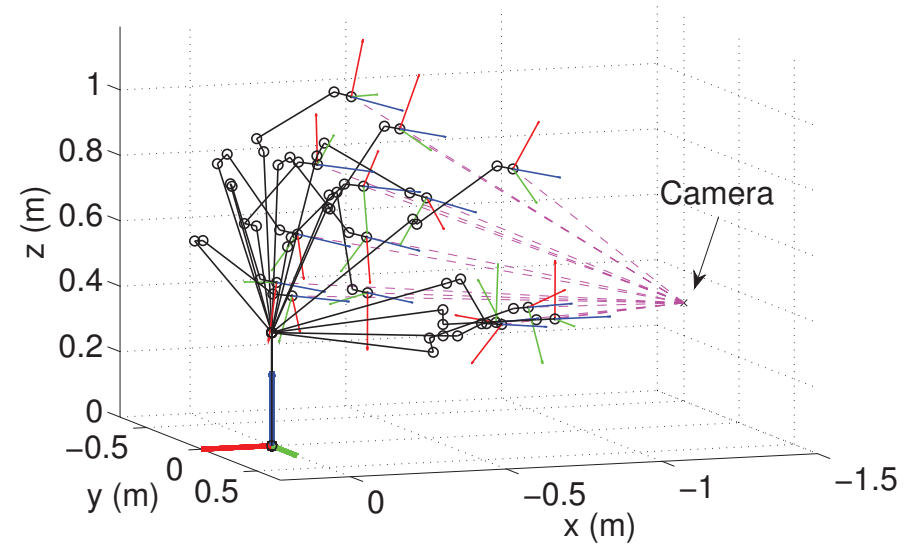

Figure 5.8: The kinematic model of the WAM in the experiment.

\section{Calibrating the WAM using the Relative Measurement Concept}

The WAM is calibrated here using the RMC, the results are summarized in Table 5.3. Many kinematic parameters $(1 \rightarrow 7)$ were identified to be under $30 \mathrm{~mm}$ and $5^{\circ}$, except for $\delta \theta_{7}$ and $\delta d_{7}$. As expected, the translational components of the manipulator base $\left(\delta a_{b}, \delta d_{b}\right.$, and $\left.\delta d_{1}\right)$ were not identified.

Table 5.3: Experimental results for the relative measurement concept.

\begin{tabular}{rrrrr}
\hline & \multicolumn{2}{c}{ Joint Errors $[\mathrm{deg}]$} & \multicolumn{2}{c}{ Link Errors $[\mathrm{mm}]$} \\
\hline$b$ & 0.3125 & -2.1665 & -0.0000 & 0.0000 \\
1 & -0.7545 & -0.6120 & 3.5969 & -0.0000 \\
2 & 0.1178 & 0.0489 & -0.4241 & 0.2090 \\
3 & 2.7705 & -0.4397 & -3.7679 & -17.6295 \\
4 & 0.5644 & -0.2677 & -1.3260 & -10.1315 \\
5 & -1.4946 & 0.8305 & 5.4388 & -24.2630 \\
6 & -3.5829 & -0.3029 & -4.2547 & -7.6065 \\
7 & 61.8989 & 4.2142 & 2.7863 & 45.8806 \\
$n$ & -55.8361 & 0.5255 & -82.9751 & 25.1906 \\
\hline
\end{tabular}


Evidently there are 36 kinematic parameters identified, meaning that ideally the full rank of $\mathbf{J}$ here should be 36 . However, the $\operatorname{rank}(\mathbf{J})$ was shown to be 31 , implying that there are 5 parameters that are linearly dependent. By removing the 5 columns of $\mathbf{J}$ associated with the parameters $\delta a_{b}, \delta d_{b}, \delta d_{1}, \delta \theta_{n}$, and $\delta d_{n}$, the $\operatorname{rank}(\mathbf{J})$ remained 31, meaning that these 5 parameters are linearly dependent. The condition number of $\mathbf{J}$ is found to be 208.88 , which means that the 31 linearly independent parameters are numerically acceptable.

An accuracy assessment is needed to evaluate the results using the RMC for the
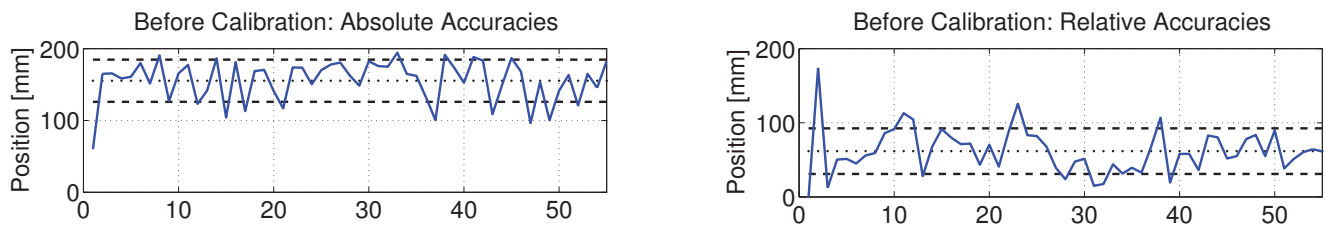

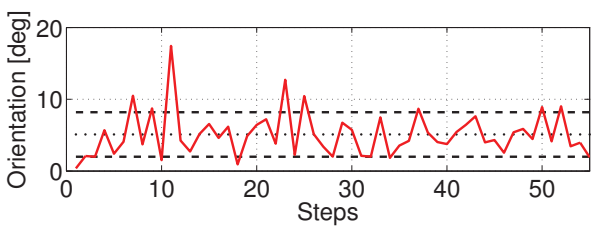

(a)

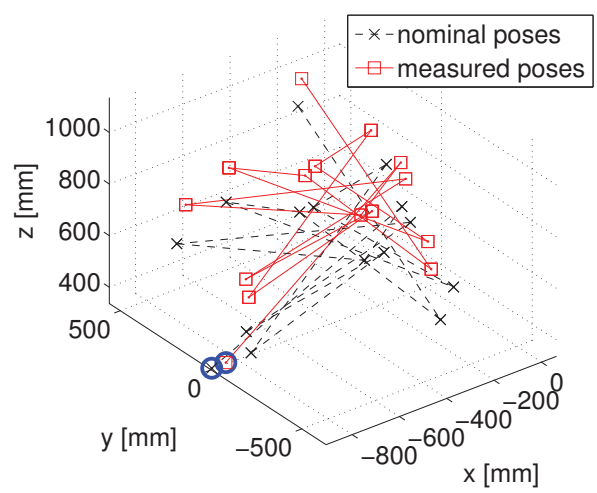

(c)

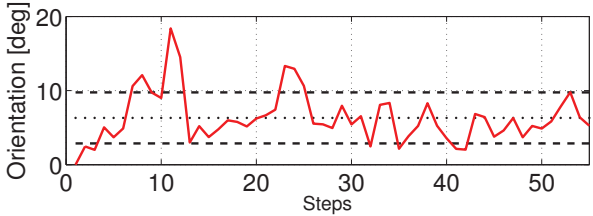

(b)

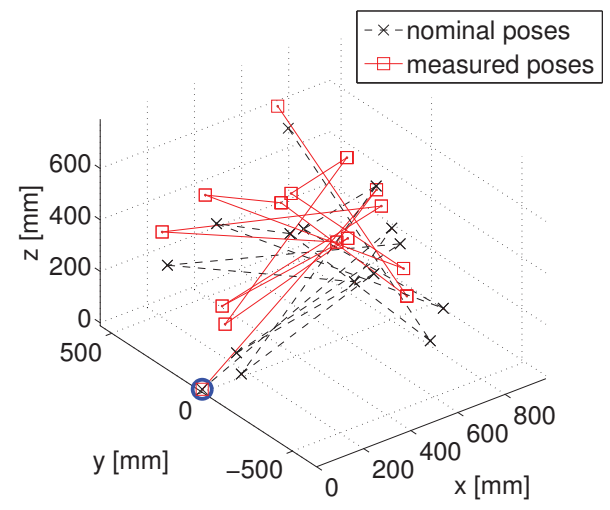

(d)

Figure 5.9: The (a) absolute and (b) relative accuracies of the WAM Arm before calibration. The first 15 (c) absolute and (d) relative poses of the WAM Arm before calibration. 

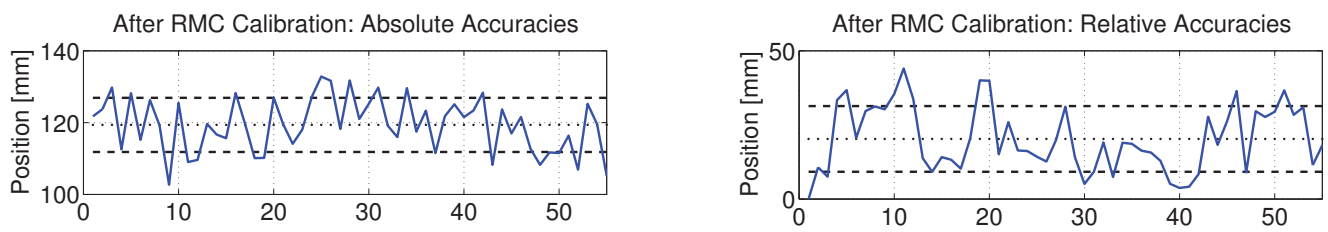

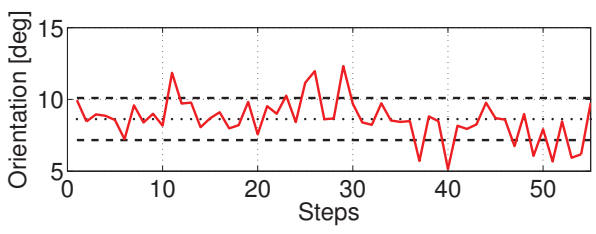

(a)

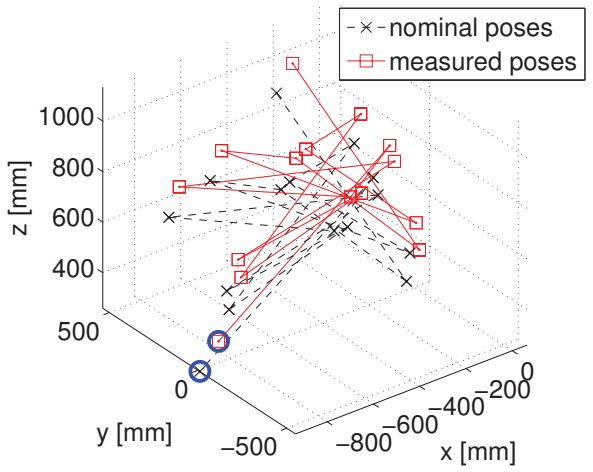

(c)

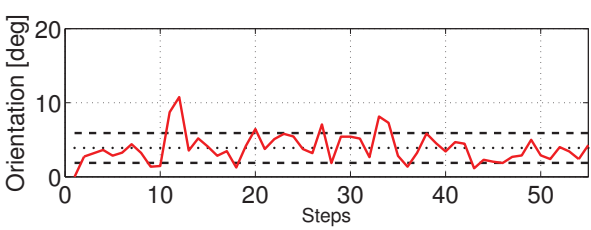

(b)

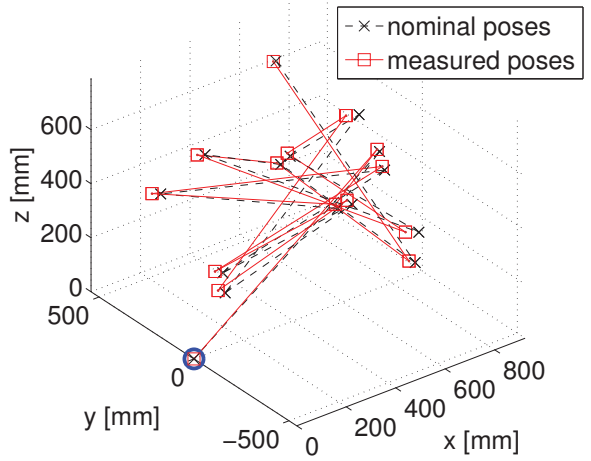

(d)

Figure 5.10: The (a) absolute and (b) relative accuracies of the WAM after RMC. The first 15 (c) absolute and (d) relative poses of the WAM after RMC.

WAM. The absolute and relative accuracies are presented in Figures 5.10(a) and 5.10(b), respectively. The first 15 absolute and relative poses after the WAM was calibrated using the RMC are presented in Figures 5.10(c) and 5.10(d), respectively. For a better comparison, the overall accuracies can be quantified using RMS errors. By the kinematic calibration using RMC, the overall absolute accuracies are improved from $\pm 7.103 \mathrm{~mm}$ and $\pm 0.268^{\circ}$ to $\pm 5.375 \mathrm{~mm}$ and $\pm 0.393^{\circ}$, respectively. The relative accuracies on the other hand improved from $\pm 3.150 \mathrm{~mm}$ and $\pm 0.329^{\circ}$ to $\pm 1.055 \mathrm{~mm}$ and $\pm 0.200^{\circ}$, respectively. 


\section{Calibrating the WAM using the Absolute Measurement Concept}

The WAM was next kinematically calibrated using the AMC, but an accurate placement and orientation of the manipulator base is required in order for this to work reliably, and now the errors in the base are included in the kinematic calibration process. Table 5.4 summarizes the results of the AMC using the WAM, many parameters were identified to be under $30 \mathrm{~mm}$ and $5^{\circ}$, but there were a few consists of higher magnitude errors (between $100 \mathrm{~mm}$ to $1000 \mathrm{~mm}$ and $10^{\circ}$ to $100^{\circ}$ for link errors and joint errors, respectively). Notice that these higher magnitude errors are only on the parameters for $b, 1,7$, and $n$, this is due to the dependencies in $b \rightarrow 1$, and $7 \rightarrow n$. The higher order errors in $b$ or 7 are compensated by a negative but equal ordered errors in 1 or $n$, respectively. The parameters $\delta \boldsymbol{a}$ and $\delta \boldsymbol{d}$ for the links $b \rightarrow 1$ had a difference greater than $50 \mathrm{~mm}$, because the initial guess of the base frame location was off by roughly that much, and the AMC algorithm corrected it.

Table 5.4: Experimental results for the absolute measurement concept.

\begin{tabular}{rrrrr}
\hline & \multicolumn{2}{c}{ Joint Errors $[\mathrm{deg}]$} & \multicolumn{2}{c}{ Link Errors $[\mathrm{mm}]$} \\
\hline$b$ & -22.5600 & -0.9658 & 52.7479 & -1794.9996 \\
1 & 21.6891 & -0.6054 & 2.6774 & 1883.2293 \\
2 & -0.3414 & 0.5268 & 3.5215 & 5.7682 \\
3 & 1.0055 & -0.3497 & -2.8524 & -19.4008 \\
4 & 0.2658 & -0.0320 & -0.0762 & -6.4809 \\
5 & -1.0366 & -0.2680 & 0.3279 & -26.0925 \\
6 & -3.3659 & -0.5238 & -0.6487 & -2.1103 \\
7 & 118.9043 & 3.5146 & 15.9245 & 1183.9726 \\
$n$ & -119.9794 & 5.2572 & -14.5426 & -1122.8103 \\
\hline
\end{tabular}



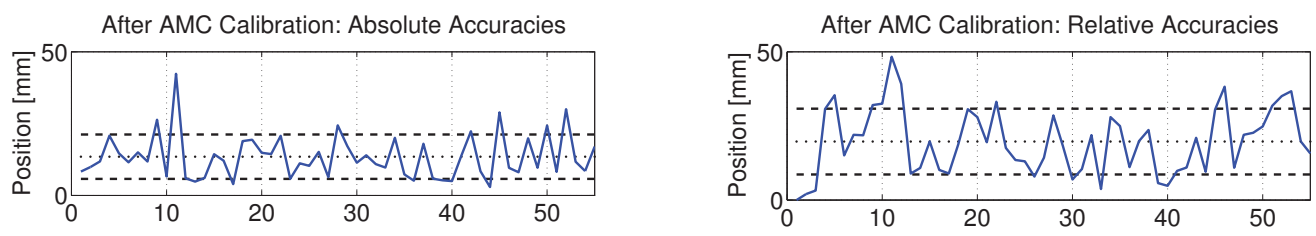

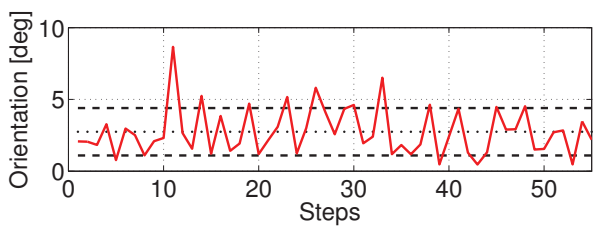

(a)

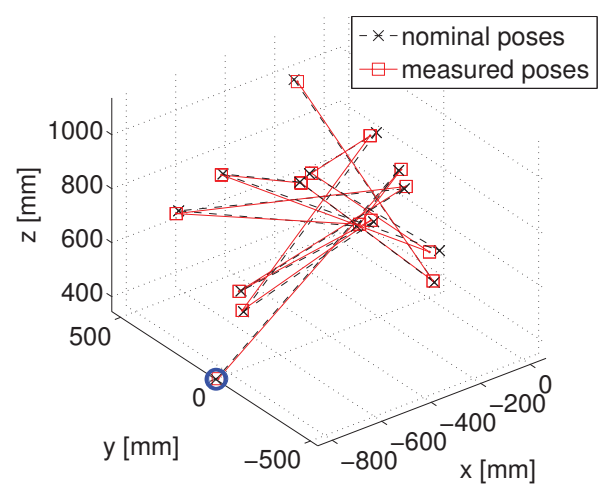

(c)

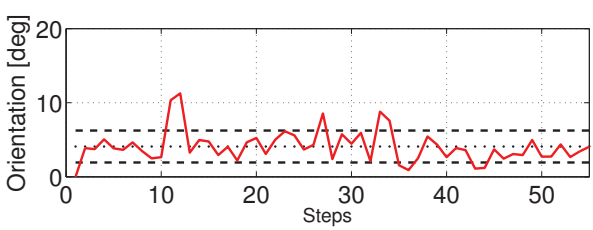

(b)

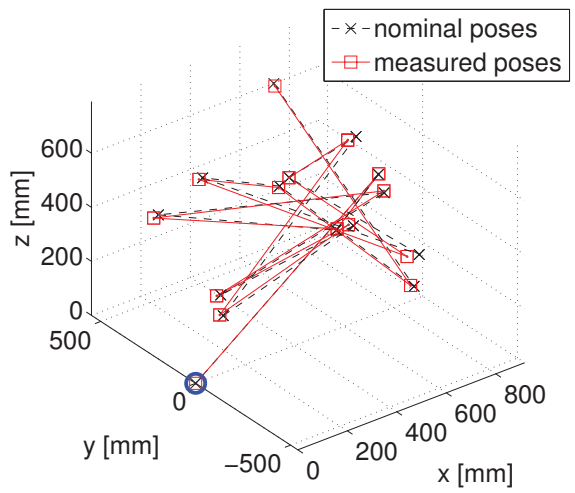

(d)

Figure 5.11: The (a) absolute and (b) relative accuracies of the WAM after AMC. The first 15 (c) absolute and (d) relative poses of the WAM after AMC.

An accuracy assessment is needed to evaluate the result of the AMC for the WAM. The absolute and relative accuracies after AMC of the WAM are presented in Figures 5.11(a) and 5.11(b). The first 15 absolute and relative poses after the WAM is calibrated using AMC are presented in Figures 5.11(c) and 5.11(d), respectively. For quantifiable comparison, the overall accuracies can be quantified using RMS errors. By the kinematic calibration using AMC, the overall absolute accuracies are improved from $\pm 7.103 \mathrm{~mm}$ and $\pm 0.268^{\circ}$ to $\pm 0.696 \mathrm{~mm}$ and $\pm 0.144^{\circ}$, respectively. The relative accuracies on the other hand improved from $\pm 3.150 \mathrm{~mm}$ and $\pm 0.329^{\circ}$ to $\pm 1.033 \mathrm{~mm}$ and $\pm 0.211^{\circ}$, respectively. 


\section{Chapter 6}

\section{Conclusion and Future Work}

\subsection{Concluding Remarks}

In this thesis a method for kinematic calibration for serial manipulators using the relative measurement concept was developed and implemented. Relative measurements were used in the kinematic calibration algorithm instead of absolute measurements. In practice, relative measurements are capable of enhancing the manipulator accuracy to the same level as absolute measurements, but with a lower cost, because it is easier to measure the relative errors rather than absolute errors.

What follows is an overview of the conclusions of each thesis chapter.

Chapter 2 Some background knowledge regarding the contents of the thesis was introduced, then some state of the art research was documented and reviewed regarding calibration methods of serial manipulators. The conclusions are:

- the use of relative measurements in the literature only focus on the position measurements rather than the pose measurements;

- the development of the relative measurement concept in this thesis shows that the orientation of the manipulator base and the measurement system must be aligned to perform properly; 
- there are many drawbacks using the DH convention as the kinematic model for calibration. There are also limitations imposed by the alternate models developed for kinematic calibration, and they also introduce more complexity. Therefore, the DH conventions are still used in this thesis, but additional parameters are introduced to address the issues;

- singular value decomposition (SVD) for approximating the pseudoinverse of the Jacobian is used for parameter identification in this thesis. Many simulations were performed, and produced similar results as the Gauss-Newton method or the Levenburg-Marquardt algorithm. Additionally, SVD allows the user to investigate the observability of the identified parameters.

Chapter 3 The first step in the kinematic calibration method is to derive a valid kinematic model. The kinematic model must include the properties of completeness, proportionality, and equivalence. The differential kinematics for the calibration can be modelled by deriving the Jacobian matrix. This chapter leads to the following conclusions:

- for useful kinematic calibration results, the parameters describing the base coordinate transformation and the end link coordinate transformation must be included in the identification;

- the kinematic calibration process using the analytical Jacobian matrix requires longer computation time; 
- the expression for the analytical Jacobian matrix can introduce representational singularities to the orientation. These singularities can be avoided by expressing the Jacobian matrix as the geometric Jacobian matrix.

Chapter 4 A simple simulation was employed to develop and validate the kinematic calibration method using relative measurements, with the absence of measurement noise in the kinematic errors. Analysis for the effects of measurement noise and pose selections were made by extending the simulation. Several points were discovered from the simulation analysis:

- the relative measurement concept can be used to correctly identify all parameters except for the ones defining the placement of the manipulator base;

- once the measurement noise is introduced, the simulation using the DH convention identified the parameters $d_{2}$ and $d_{3}$ poorly. However, those parameters were better identified once the Hayati parameter was introduced to the model;

- based on the simulation that relates the identifiability to the required measurement poses, it was found that at least 30 to 35 poses are required to acquire the same level of identification accuracy between the no noise situation and the presence of measurement noise;

- finally, the simulation to determine the best poses for kinematic calibration was performed. It was found that the pose trajectory generated randomly within the $E_{3}$ space produced the best kinematic calibration result using both absolute and relative measurements.

Chapter 5 Experiments for validating the kinematic calibration using the relative measurement concept were reported here. Two manipulators were used for the calibration: the Thermo CRS A465; and the 7 DOF WAM Arm. The end-effector 
pose can be measured using the novel camera calibration algorithm developed with OpenCV. The results lead to the following conclusions:

- the camera calibration algorithm was able to reproject the points in the image with an RMS value of roughly $0.22 \mathrm{px}$. for the $720 \mathrm{p}$ cameras;

- the experiment using the A465 was performed with planar trajectory on the xy-plane with the end-effector pointing down. The calibration algorithm did not converge;

- the experiment using the WAM Arm was performed with the volumetric trajectory with the randomly generated end-effector positions in $E_{3}$ with the endeffector facing towards the camera. The calibration algorithm converged;

- the WAM Arm was calibrated using both absolute and relative measurement algorithms. The results showed that both methods produced similar relative pose accuracy (in terms of RMS values).

\subsection{Suggestions for Future Work}

- As the main objective of this thesis was to develop and validate the use of relative measurements for kinematic calibration, methods to reach the desirable accuracy in the end-effector pose measurements was neglected. Improving the accuracy of the measurements would likely improve the kinematic calibration results.

- The non-geometric effects on the end-effector errors should be modelled and included in the calibration procedure. 
- The precise knowledge of the manipulator base orientation was required in the current development of the RMC, just like the AMC, due to the Jacobian matrix used here. To avoid the need to do this, a new differential kinematic relationship is needed by deriving from Eqs. (A.26a) and (A.26b). Due to the equations being highly nonlinear, the gradient vector of the system can be quite complex to obtain analytically. Techniques for approximating this gradient vector should be further investigated. This method would have the potential to produce more accurate kinematic calibration results, with the current setup in this thesis. 


\section{List of References}

[1] R. P. Judd, and A. B. Knasinski. A technique to calibrate industrial robots with experimental verification. In Proc. of 1987 IEEE International Conference on Robotics and Automation, pp. 183-189, April 1987.

[2] C. Wu. The Kinematic Error Model for the Design of Robot Manipulators. In Proc. of the American Control Conference, San Francisco, Ca, pp. 497-502, 1983.

[3] M. J. D. Hayes and P. L. O'Leary. Kinematic Calibration Procedure for Serial Robots with Revolute Axes. Institute for Automation, University of Leoben, April 12, 2001.

[4] N. W. Simpson. Kinematic calibration of 6-axis serial robots using the relative measurement concept. Carleton University, 2004.

[5] In-Chul, Ha, Kinematic parameter calibration method for industrial robot manipulator using the relative position, Institute of Industrial Technology 103-28, Mounji-dong, Yuseong-gu, Daejeon 305-380, Korea, 2008.

[6] C. C. D. Lu, and M.J.D. Hayes, May 30-31, 2013, "Kinematic Calibration of 6R Serial Manipulators Using Relative Measurements", Proceedings of the 8th CCToMM Symposium on Mechanisms, Machines, and Mechatronics, cole de technologie suprieure, Montral, QC, Canada.

[7] Z. S. Roth, B. W. Mooring, and B. Ravani. An overview of robot calibration. IEEE J. Robotics Automat., pages 1690-1695, Scottsdale, AZ, 1989.

[8] J. S. Shamma, and D. E. Whitney. A Method for Inverse Robot Calibration. Transactions of the ASME Journal of Dynamic Systems, Measurements, and Control, vol. 109, pp. 36-43, 1987. 
[9] A. Doria, F. Angrilli, and S. de Marchi. Inverse Kinematics Robot Calibration by Spline Functions. Applied Mathematical Modelling, 17(9): 492-498, September 1993.

[10] X. Zhong, J. Lewis, and F. L. N-Nagy. Inverse Robot Calibration using Artificial Neural Networks. Engineering Applications and Artificial Intelligence. Vol. 9, No. 1, pp. 83-93,1996.

[11] J. U. Dolinsky. The development of a genetic programming Method for kinematic robot calibration. Liverpool John Moores University, 2001.

[12] B. W. Mooring, Z. S. Roth, and M. R. Driels. Fundamentals of Manipulator Calibration. John Wiley Sons, Inc., 1991.

[13] H. W. Stone. Kinematic Modeling, Identification, and Control of Robotic Manipulators. Kluwer Academic Publishers, Boston, 1987.

[14] M. E. Sklar. Metrology and Calibration Techniques for the Performance Enhancement of Industrial Robots. PhD. thesis, University of Texas at Austin, Austin, Texas, 1988.

[15] M. E. Sklar. Geometric calibration of industrial manipulators by circle point analysis. In Proceedings of Second Conference on Recent Advances in Robotics, pp. 178-202, Florida Atlantic University, May, 1989.

[16] M. S. Kim, H. S. Yoo, S. W. Cho, H. S. Change, and G. Spur. A New Calibration Method. Annals of the CIRP. Vol. 39. January, 1990.

[17] C. Gong, J. Yuan, and J. Ni. A Self-Calibration Method for Robotic Measurement System. Journal of Manufacturing Science and Engineering, 122(1):174$181,2000$.

[18] D. E. Whitney, C. A. Lozinski, and J. M. Rourke. Industrial Robot Forward Calibration Methods and Results, ASME Journal of Dynamic Systems, Measurement and Control, Vol. 108, No. 1.

[19] J Chen, and L. M. Chao. Positioning error analysis for robot manipulators with all rotary joints. In Proceedings of 1986 IEEE Conference on Robotics and Automation. pp. 1011 - 1016, April 1986.

[20] J. F. Jarvis. Microsurveying: Towards robot accuracy. In Proceedings of 1987 IEEE Conference on Robotics and Automation. pp. 175 - 182, April 1987. 
[21] H. Zhuang, W. C. Wu, and Z. S. Roth. "Camera-assisted calibration of SCARA arms", Proceedings 1995 IEEE/RSJ International Conference on Intelligent Robots and Systems. pp. 507-512, vol. 1, 1995.

[22] H. Zhuang, Z. S. Roth, X. Xu, and K. Wang. "Camera calibration issues in robot calibration with eye-on-hand configuration", Robotics and Computer-Integrated Manufacturing. pp. 401-412, vol. 10, 1994.

[23] K. Lenz, and R. Y. Tsai. "Calibrating a Cartesian robot with eye-on-hand configuration independent of eye-to-hand relationship". IEEE Trans. PAMI 11(9): 916-928, 1989.

[24] Y. Xiong. Vision Based Automatic Calibration of Microrobotic System. Master's Thesis, Tampere University of Technology, 2012.

[25] A. Nubiola, M. Slamani, and I. A. Bonev. A new method for measuring a large set of poses with a single telescoping ballbar. Published by Elsevier Inc., 2012.

[26] J. Ziegert, and P. Dateris. Basic Considerations for Robot Calibration. IEEE Transactions on Robotics and Automation. pp. 932-938. 1988.

[27] S. Hayati and M. Mirmirani. A software for robot parameter estimation. SME Paper \# MS84-1052, presented at Robots West Conference, Anaheim, CA, November, 1984.

[28] W. K. Veitschegger and C. H. Wu. Robot accuracy analysis based on kinematics. IEEE Journal of Robotics and Automation, RA-2(3): 171-179, Sept. 1986.

[29] H. W. Stone, and A. C. Sander. "A Prototype Arm Signature Identification System". Proceedings of the 1987 IEEE International Conference on Robotics and Automation. pp. 175-182, 1987.

[30] B. W. Mooring. The effect of joint axis misalignment on robot positioning accuracy. In Proceedings of the 1983 ASME Computers in Engineering Conference, pp. 151-155. August, 1983.

[31] J. Denavit and R. S. Hartenburg. A kinematic notation for lower-pair mechanisms based on matrices. Transactions of ASME-Journal of Applied Mechanics, 22(2):215-221, June 1955.

[32] J. J. Craig. Introduction to Robotics Mechanics and Control. Addison Wesley, Reading, Massachusetts, 1986. 
[33] J. Shigley and J. Uicker. Theory of Machines and Mechanisms. New York: McGraw-Hill, Inc., 1995.

[34] M. W. Spong, and M. Vidyasagar. Robot Dynamics and Control. N. Y., John Wiley \& Sons, 1989.

[35] R. P. Paul. Robot Manipulators: Mathematics, Programming, and Control. Cambridge, Mass, MIT Press, 1981.

[36] D. G. Luenberger, Linear and Nonlinear Programming, Chap. 7, Addison-Wesley, MA, 1984.

[37] S. M. Goldfeld, R. E. Quandt, and H. F. Trotter, Maximization by quadratic hill-climbing, Econometrica, vol. 34, pp. 541551, 1966.

[38] P. B. Zwart, Nonlinear Programming: A Quadratic Analysis of Ridge Paralysis, Washington University, Report COO-1493-21, St. Louis, Mo., Jan. 1969.

[39] A. Matthews, and D. Davies, "A Comparison of Modified Newton Methods for Unconstrained Optimization," Computer Journal, vol. 14, pp. 293-294, 1971.

[40] K. Levenburg. A Method for the Solution of Certain Problems in Least Squares. Quart. Appl. Math., 2:164-168, 1944.

[41] D. Marquardt. An Algorithm for Least-Squares Estimation of Nonlinear Parameters. SIAM Journal of Applied Mathematics, 11:431-441, 1963.

[42] T. Weise. Global Optimization Algortihm - Theory and Application -. http://www.it-weise.da/.

[43] S. Aoyagi. Selection of optimal measuring points using genetic algorithm in the process to calibrate robot kinematic parameters. Technical Report. Kansai University, Japan.

[44] H. Zhuang, J. Wu, and W. Huang. Optimal planning of robot calibration experiments by genetic algorithms. In Proceedings of the 1996 IEEE International Conference on Robotics and Automation. Minneapolis, Minnesota. April, 1996.

[45] William H. Press, Saul A. Teukolsky, William T. Vetterling, and Brian P. Flannery. Numerical Recipes in C: The Art of Scientific Computing. Cambridge University Press, $2^{\text {nd }}$ edition, 1992. 
[46] G. E. Forsythe and C. B. Moler. Computer Solution of Linear Algebraic Systems. Prentice Hall, Englewood Cliffs, NJ, 1967.

[47] L. J. Everett, M. R. Driels, and B. M. Mooring. Kinematic modelling for robot calibration. IEEE International Conference on Robotics and Automation, Vol. 1, pp.183-189, 1987.

[48] B. Siciliano, L. Sciavicco, L. Villani, and G. Oriolo. Robotics Modelling, Planning, and Control. London: Springer-Verlag London Limited, 1995.

[49] R. N. Jazar. Theory of Applied Robotics Kinematics, Dynamics, and Control. Springer Science+Business Media, LLC, 2007.

[50] Thermo CRS, Ltd. A465 Robot System Guide. July 2002.

[51] Barrett Technology Inc.. A465 Robot System Guide. July 2002.

[52] G. Bradski, and A. Kaehler. Learning OpenCV. Published by O'Reily Media Inc., 2005 . 


\section{Appendix A}

\section{Spatial Displacements Representations}

A manipulator can be schematically represented from a mechanical viewpoint as a kinematic chain of rigid bodies (linkages) connected with joints. The pose of the rigid bodies can be represented in the form of coordinate frames. Consider two points in $E_{3}$ Euclidean space, $\boldsymbol{p}_{o}$ and $\boldsymbol{p}_{i}$, as illustrated in Figure A.1. The point $\boldsymbol{p}_{i}$ can be transformed from $\boldsymbol{p}_{o}$ using

$$
\boldsymbol{p}_{i}=\boldsymbol{R} \boldsymbol{p}_{o}+\boldsymbol{t}
$$

where $\boldsymbol{p}_{o}$ and $\boldsymbol{p}_{i}$ represent the Cartesian location of $O_{o}$ and $O_{i}$, respectively. $\boldsymbol{R}$ is a $3 \times 3$ orthonormal matrix representing the orientation of $\Sigma_{i}$ from $\Sigma_{o}$, and $\boldsymbol{t}$ is a $3 \times 1$ vector representing the translation of $\Sigma_{i}$ from $\Sigma_{o}$. The point $\boldsymbol{p}_{i}$ is expressed by

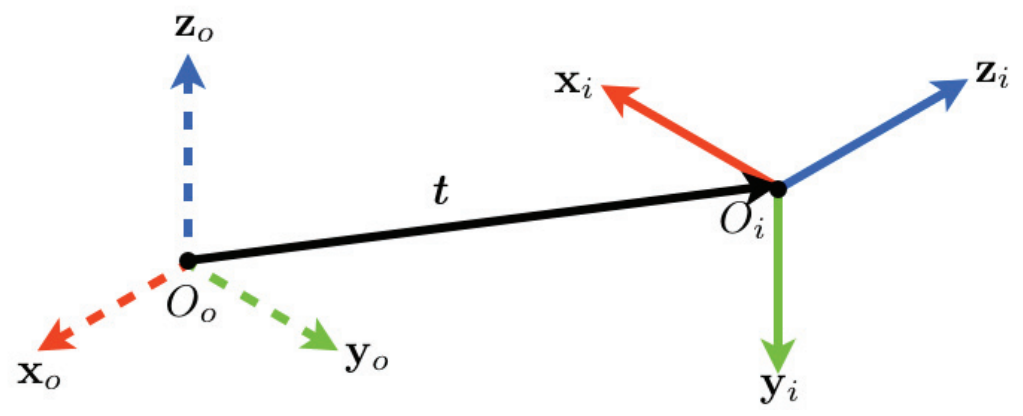

Figure A.1: Coordinate system representation of a rigid body 
rotating $\Sigma_{o}$ by $\boldsymbol{R}$, then translate $\boldsymbol{p}_{o}$ by $\boldsymbol{t}$ to reach that point. This procedure could become a problem if consecutive transformations are needed, which is needed in robot kinematic problems, computer vision/graphics problems, and more. This particular issue can be overcome by introducing homogeneous coordinates to define all points.

\section{A.1 Rotation of a Rigid Body}

There is a fundamental problem in representing rotations of a rigid body in $E_{3}$ Euclidean space, because spatial rotations do not topologically allow a smooth mapping in $E_{3}$. Imagine trying to wrap a ball with a piece of paper, there are parts of the paper overlapping in order to make the sphere. This problem is precisely the reason why singularities occur in some rotations, expressed with minimal representations.

There are many different method of representing the rotations of a rigid body in $E_{3}$, there are methods with three-parameter, four-parameter, or more. The threeparameter methods may be the minimal representation of rotations but at least one parameter can become singular (undefined) causing the other two to be linearly dependent. The four-parameter or more methods are defined to overcome the singularity issue with the cost of introducing redundant parameters in the method.

The choice of methods to represent rotations depends on the application and the method of computation. The redundant parameters is not a problem for most computer applications, so those algorithms use representations with extra parameters except for numerical algorithms. However, engineers prefer to work with the minimum required parameters, and kinematic calibration problem involves numerical algorithms to search for kinematic parameters. 


\section{A.1.1 Rotation Matrix}

The rotation matrix representation is for many reasons the most useful representation method of spatial rotations. It is derived by determining the direction cosines (concatenating three unit vectors, representing the three directions of the axes of $\Sigma$ ), providing the orthonormal property. It has the form of

$$
\boldsymbol{R}=\left[\begin{array}{lll}
r_{11} & r_{12} & r_{13} \\
r_{21} & r_{22} & r_{23} \\
r_{31} & r_{32} & r_{33}
\end{array}\right]
$$

There are several advantages using this method, and they are mostly due to the orthonormal property of the expression. First is the compositions of rotations experienced by a rigid body can be equivalently computed using matrix multiplication. Second, the inverse of the rotation matrix is equivalent to its transpose.

The primary disadvantage of this method is due to the overly redundant representation. First, the matrix makes it hard to interpret the rotations of the rigid body in $E_{3}$. Second, the redundancies in the representation can introduce linear dependancies, which compromises the solution search using numerical methods.

\section{A.1.2 Euler Angles}

The 9 parameters characterizing the rotation matrix are not all independent but related by 6 constraints due to the orthogonality conditions. This implies that only 3 independent parameters are needed to describe orientation of a rigid body in $E_{3}$, and these parameters constitute a minimal representation. Therefore, a set of three angles $\boldsymbol{\Phi}=[\alpha \beta \gamma]^{T}$ can be extracted directly from $\boldsymbol{R}$. 
Knowing that the rotation matrix express the elementary rotation about one of the coordinate axes as a function of one angle, this is reviewed later in Appendix A.3.1. Then, a generic rotation matrix can be obtained by composing three sequential elementary rotations while avoiding the consecutive rotations about the same axis. Then, 12 out of the 27 possible combinations define each possible set of Euler angles.

Each set of Euler angles exhibit a common advantage and a common disadvantage. The advantage is that the Euler angles are easier to visualize comparing to any other methods. And the disadvantage is that, although the properties may be different, there always exist the orientations that cause singularity in one of the three Euler angles. The choice of the Euler angles depend on the applications of use, where two most commonly used set are reviewed here: the ZYZ angles and the ZYX angles.

\section{ZYZ Angles}

The rotation describing the ZYZ angles is extracted with the composition of the following sequential elementary rotations,

$$
\boldsymbol{R}(\boldsymbol{\Phi})=\boldsymbol{R}_{z}(\alpha) \boldsymbol{R}_{y}(\beta) \boldsymbol{R}_{z}(\gamma)=\left[\begin{array}{ccc}
c_{\alpha} c_{\beta} c_{\gamma}-s_{\alpha} s_{\gamma} & -c_{\alpha} c_{\beta} s_{\gamma}-s_{\alpha} c_{\gamma} & c_{\alpha} s_{\beta} \\
s_{\alpha} c_{\beta} c_{\gamma}+c_{\alpha} s_{\gamma} & -s_{\alpha} c_{\beta} s_{\gamma}+c_{\alpha} c_{\gamma} & s_{\alpha} s_{\beta} \\
-s_{\beta} c_{\gamma} & s_{\beta} s_{\gamma} & c_{\beta}
\end{array}\right] .
$$

The Euler angles are extracted (assuming $r_{13} \neq 0$ and $r_{23} \neq 0$ ) to be

$$
\begin{aligned}
& \alpha=\arctan 2\left(r_{23}, r_{13}\right), \\
& \beta=\arctan 2\left(\sqrt{r_{13}^{2}+r_{23}^{2}}, r_{33}\right), \\
& \gamma=\arctan 2\left(r_{32},-r_{31}\right),
\end{aligned}
$$


for $0<\beta<\pi$, and

$$
\begin{aligned}
& \alpha=\arctan 2\left(-r_{23},-r_{13}\right), \\
& \beta=\arctan 2\left(-\sqrt{r_{13}^{2}+r_{23}^{2}}, r_{33}\right), \\
& \gamma=\arctan 2\left(-r_{32}, r_{31}\right),
\end{aligned}
$$

for $-\pi<\beta<0$. When $\sin \beta=0$, it is only possible to determine the sum or the difference of $\alpha$ and $\gamma$ (they became dependent, with infinite solutions).

\section{ZYX Angles}

The ZYX angles, also known as the roll-pitch-yaw angles, is the common choice of representation in the applications of the aeronautical field. This set of angles can be extracted directly from the following sequential rotations

$$
\boldsymbol{R}(\boldsymbol{\Phi})=\boldsymbol{R}_{z}(\alpha) \boldsymbol{R}_{y}(\beta) \boldsymbol{R}_{x}(\gamma)=\left[\begin{array}{ccc}
c_{\alpha} c_{\beta} & c_{\alpha} s_{\beta} s_{\gamma}-s_{\alpha} c_{\gamma} & c_{\alpha} s_{\beta} c_{\gamma}+s_{\alpha} s_{\gamma} \\
s_{\alpha} c_{\beta} & s_{\alpha} s_{\beta} s_{\gamma}+c_{\alpha} c_{\gamma} & s_{\alpha} s_{\beta} c_{\gamma}-c_{\alpha} s_{\gamma} \\
-s_{\beta} & c_{\beta} s_{\gamma} & c_{\beta} c_{\gamma}
\end{array}\right]
$$

The roll-pitch-yaw angles can be extracted to be

$$
\begin{aligned}
& \alpha=\arctan 2\left(r_{21}, r_{11}\right), \\
& \beta=\arctan 2\left(-r_{31}, \sqrt{r_{32}^{2}+r_{33}^{2}}\right), \\
& \gamma=\arctan 2\left(r_{32}, r_{33}\right),
\end{aligned}
$$

for $-\pi / 2<\beta<\pi / 2$, and 


$$
\begin{aligned}
& \alpha=\arctan 2\left(-r_{21},-r_{11}\right), \\
& \beta=\arctan 2\left(-r_{31},-\sqrt{r_{32}^{2}+r_{33}^{2}}\right), \\
& \gamma=\arctan 2\left(-r_{32},-r_{33}\right),
\end{aligned}
$$

for $\pi / 2<\beta<3 \pi / 2$. The same singularity problem surfaced in this case, except it is when $\cos \beta=0$, only the sum or the difference of $\alpha$ and $\gamma$ can be determined.

\section{A.1.3 Angle-Axis}

Angle-axis representation is described by the Rodriguez equation, it is a direct result of the Euler rigid body rotation theorem. In this method, a rotation is described by the magnitude of rotation $\Phi$ with the positive right-hand direction about the unit vector $\hat{\boldsymbol{u}}$. This method can also be expressed in the form of a rotation matrix by applying several elementary rotations (of course some conditions of the rotation

matrix must be established first to derive the relationship between $\left[\theta_{x}, \theta_{y}, \theta_{z}\right]^{T}$ and $\left.[\hat{\boldsymbol{u}}, \Phi]^{T}\right)$,

$$
\begin{aligned}
\boldsymbol{R}(\hat{\boldsymbol{u}}, \Phi)= & \boldsymbol{R}_{z}\left(\theta_{z}\right) \boldsymbol{R}_{y}\left(\theta_{y}\right) \boldsymbol{R}_{z}(\Phi) \boldsymbol{R}_{y}\left(-\theta_{y}\right) \boldsymbol{R}_{z}\left(-\theta_{z}\right), \\
= & {\left[\begin{array}{ccc}
u_{1}^{2} \text { vers } \Phi+c_{\Phi} & u_{1} u_{2} \text { vers } \Phi-u_{3} s_{\Phi} & u_{1} u_{3} \text { vers } \Phi+u_{2} s_{\Phi} \\
u_{1} u_{2} \operatorname{vers} \Phi+u_{3} s_{\Phi} & u_{2}^{2} \text { vers } \Phi+c_{\Phi} & u_{2} u_{3} \text { vers } \Phi-u_{1} s_{\Phi} \\
u_{1} u_{3} \text { vers } \Phi-u_{2} s_{\Phi} & u_{2} u_{3} \text { vers } \Phi+u_{1} s_{\Phi} & u_{3}^{2} \text { vers } \Phi+c_{\Phi}
\end{array}\right], }
\end{aligned}
$$

where

$$
\text { vers } \Phi=2 \sin ^{2} \frac{\Phi}{2}=1-\cos \Phi .
$$


The parameters in the angle-axis representation can be computed as

$$
\begin{gathered}
\Phi=\cos ^{-1}\left(\frac{\operatorname{trace}(\mathbf{R})-1}{2}\right), \\
\hat{\boldsymbol{u}}=\frac{1}{2 \sin \Phi}\left[\begin{array}{c}
r_{32}-r_{23} \\
r_{13}-r_{31} \\
r_{21}-r_{12}
\end{array}\right] .
\end{gathered}
$$

It can be observed that the three components forming $\hat{\boldsymbol{u}}$ are not linearly independent, but instead are constrained by the condition

$$
u_{1}^{2}+u_{2}^{2}+u_{3}^{2}=1
$$

Another observation is when $\sin \Phi=0$ then Eq. (A.10b) becomes undefined, meaning $\hat{\boldsymbol{u}}$ is arbitrary (singularity).

\section{A.1.4 Unit Quaternion}

The unit quaternion is used to represent rotations using an ordered quadruple of real numbers, $\mathcal{P}=\left(p_{0}, p_{1}, p_{2}, p_{3}\right)$, with $p_{0}$ being the scalar component and $\left[p_{1}, p_{2}, p_{3}\right]^{T}$ being the vector component. The unit quaternion is constrained by the condition

$$
p_{0}^{2}+p_{1}^{2}+p_{2}^{2}+p_{3}^{2}=1
$$

where the parameters $p_{i}$ for $i=0, \ldots, 3$ are known as the Euler-Rodrigues parameters.

Like the rotation matrices, quaternion is capable of expressing compositing rotations in a convenient form, but some quaternion operations must be defined first. 
Let's introduce another quadruples of real numbers $\mathcal{Q}=\left(q_{0}, q_{1}, q_{2}, q_{3}\right)$. The addition and subtraction for the quaternions are performed component wise, where as the multiplication is defined by

$$
\begin{array}{r}
\mathcal{P} * \mathcal{Q}=\left(p_{0} q_{0}-p_{1} q_{1}-p_{2} q_{2}-p_{3} q_{3}, p_{0} q_{1}+p_{1} q_{0}+p_{2} q_{3}-p_{3} q_{2},\right. \\
\left.p_{0} q_{2}-p_{1} q_{3}+p_{2} q_{0}+p_{3} q_{1}, p_{0} q_{3}+p_{1} q_{2}-p_{2} q_{1}+p_{3} q_{0}\right) .
\end{array}
$$

The quaternion representation can also be expressed in a flag form

$$
\mathcal{P}=p_{0}+p_{1} \boldsymbol{i}+p_{2} \boldsymbol{j}+p_{3} \boldsymbol{k}=p_{0}+\mathbf{p},
$$

where $\boldsymbol{i}, \boldsymbol{j}, \boldsymbol{k}$ are flags and have the following special product properties:

$$
\begin{aligned}
& \boldsymbol{i}^{2}=\boldsymbol{j}^{2}=\boldsymbol{k}^{2}=\boldsymbol{i j} \boldsymbol{k}=-1 \\
& \boldsymbol{j} \boldsymbol{k}=-\boldsymbol{k} \boldsymbol{j}=\boldsymbol{i} ; \quad \boldsymbol{k} \boldsymbol{i}=-\boldsymbol{i} \boldsymbol{k}=\boldsymbol{j} ; \quad \boldsymbol{i j}=-\boldsymbol{j} \boldsymbol{i}=\boldsymbol{k} .
\end{aligned}
$$

The quaternion $\mathcal{P}$ has a conjugate $\mathcal{P}^{*}$, and is defined as

$$
\mathcal{P}^{*}=p_{0}-p_{1} \boldsymbol{i}-p_{2} \boldsymbol{j}-p_{3} \boldsymbol{k}=p_{0}-\mathbf{p} .
$$

Therefore,

$$
\begin{aligned}
& \|\mathcal{P}\|=\sqrt{\mathcal{P} \mathcal{P}^{*}}=\sqrt{p_{0}^{2}+p_{1}^{2}+p_{2}^{2}+p_{3}^{2}}, \\
& \mathcal{P}^{-1}=\frac{1}{\mathcal{P}}=\frac{\mathcal{P}^{*}}{\|\mathcal{P}\|^{2}} .
\end{aligned}
$$

For a unit quaternion $\mathcal{P},\|\mathcal{P}\|=1$ yielding $\mathcal{P}^{-1}=\mathcal{P}^{*}$. 
The unit quaternion $\mathcal{P}$ can be expressed in terms of the parameters in the angleaxis representation

$$
\mathcal{P}(\hat{\boldsymbol{u}}, \Phi)=p_{0}+\mathbf{p}=\cos \frac{\Phi}{2}+\sin \frac{\Phi}{2} \hat{\boldsymbol{u}}
$$

By substituting Eq. (A.17) into Eq. (A.9), the rotation matrix can be expressed in terms of the Euler-Rodrigues parameters,

$$
\boldsymbol{R}(\mathcal{P})=\left[\begin{array}{lll}
2\left(p_{0}^{2}+p_{1}^{2}\right)-1 & 2\left(p_{1} p_{2}-p_{0} p_{3}\right) & 2\left(p_{1} p_{3}+p_{0} p_{2}\right) \\
2\left(p_{1} p_{2}+p_{0} p_{3}\right) & 2\left(p_{0}^{2}+p_{2}^{2}\right)-1 & 2\left(p_{2} p_{3}-p_{0} p_{1}\right) \\
2\left(p_{1} p_{3}-p_{0} p_{2}\right) & 2\left(p_{2} p_{3}+p_{0} p_{1}\right) & 2\left(p_{0}^{2}+p_{3}^{2}\right)-1
\end{array}\right]
$$

\section{A.2 Homogeneous Coordinates}

Consider two points on the same line in this $E_{3}$ space illustrated in Figure A.2. The point $\boldsymbol{p}_{1}$ can be described by the Cartesian coordinates $\left(p_{x}, p_{y}, p_{z}\right)$ and the point $\boldsymbol{p}_{2}$ is described by $\left(\mu p_{x}, \mu p_{y}, \mu p z\right)$. If $\mu \rightarrow \infty$ in Cartesian coordinate system, the point $\boldsymbol{p}_{2}$ becomes seemingly pointless $(\infty, \infty, \infty)$. However, if homogeneous coordinates are introduced, it can be characterized as a point at infinity. The homogenizing coordinate is introduced such that

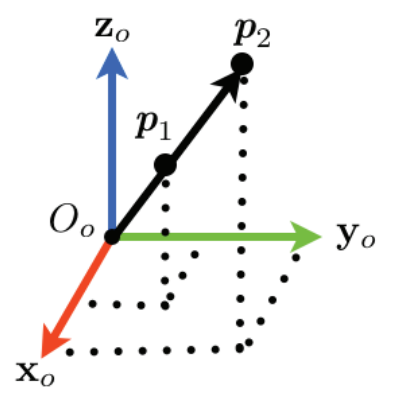

Figure A.2: Coordinate system representation of a point 


$$
p_{x}=\frac{x_{1}}{w}, p_{y}=\frac{x_{2}}{w}, p_{z}=\frac{x_{3}}{w}
$$

where $w$ is the homogenizing coordinate and $w \neq 0$. The homogeneous coordinates of $\boldsymbol{p}_{1}$ and $\boldsymbol{p}_{2}$ may now be written as the ratios $\left(x_{1}: x_{2}: x_{3}: w\right)$.

By representing the points using homogeneous coordinates, the Cartesian coordinates $\boldsymbol{p}_{1}$ is recovered if $w=1$ and $\boldsymbol{p}_{2}$ is recovered if $w=\frac{1}{\mu}$. If two proportional sets of homogeneous coordinates are given, $\left(x_{1}: x_{2}: x_{3}: w\right)$ and $\left(\lambda x_{1}: \lambda x_{2}: \lambda x_{3}: \lambda w\right)$, where $\lambda=0$ still represent the same point in space. If $w \rightarrow 0$, this is similar to when $\mu \rightarrow \pm \infty$ in Cartesian coordinates, then the point at infinity on this same line can be characterized.

\section{A.3 Homogeneous Transformation}

Consider Figure $A .1$ again, if the points, $\boldsymbol{p}_{o}$ and $\boldsymbol{p}_{i}$, are both expressed in terms of the homogeneous coordinates, then it is possible to represent translation, rotation, and scaling as a single $4 \times 4$ homogeneous transformation matrix

$$
\left[\begin{array}{c}
p_{i, x} \\
p_{i, y} \\
p_{i, z} \\
w
\end{array}\right]=\left[\begin{array}{c|c}
\boldsymbol{R}_{3 \times 3} & \boldsymbol{t}_{3 \times 1} \\
---------- & ---------- \\
\boldsymbol{P}_{1 \times 3} & \mid
\end{array}\right]\left[\begin{array}{c}
p_{o, x} \\
p_{o, y} \\
p_{o, z} \\
w
\end{array}\right],
$$

where $\boldsymbol{P}_{1 \times 3}$ is the perspective transform that is usually a zero vector in manipulator kinematics, and $W_{1 \times 1}$ is the scaling factor that is usually 1 . 


\section{A.3.1 3D Pose Transformation}

In this section, several elementary transformations of the coordinate frames are summarized. These transformations are also termed the 3D pose transformation.

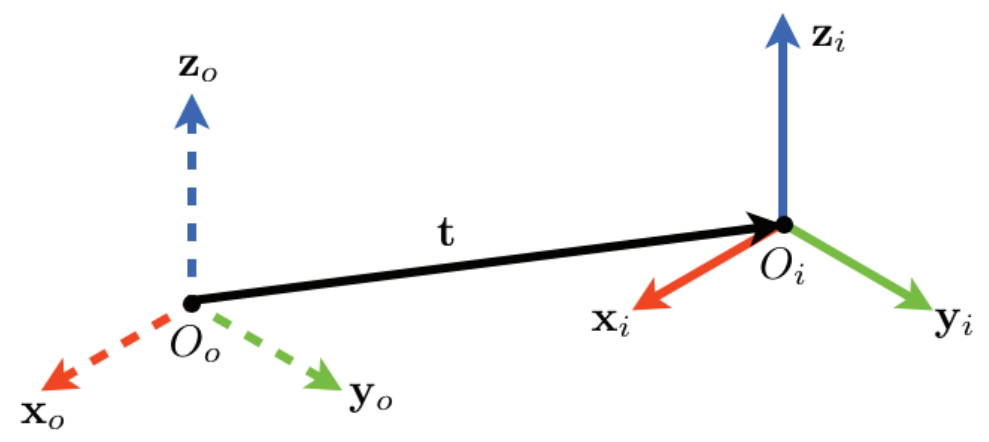

(a)

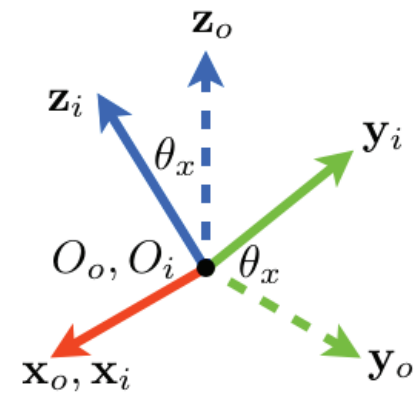

(b)

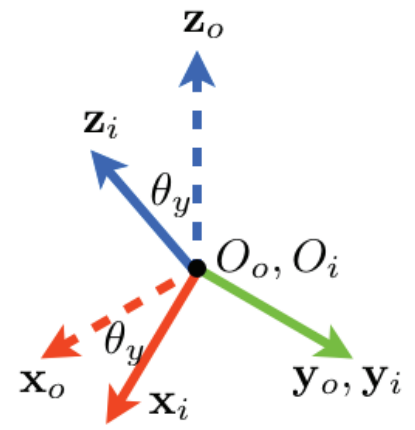

(c)

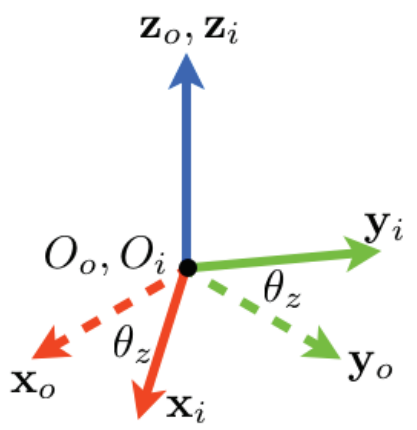

(d)

Figure A.3: Transformations between coordinate frames. (a) Pure translation, and pure rotation about the corresponding axes (b) $\mathbf{x}$-axis, (b) $\mathbf{y}$-axis and (d) $\mathbf{z}$-axis

Consider the pure translational case as illustrated in Figure A.3(a), the point $\boldsymbol{p}_{i}$ is obtained in the Cartesian form as

$$
\boldsymbol{p}_{i}=\boldsymbol{p}_{o}+\boldsymbol{t}
$$

Notice that there is no rotational displacements between the frames, resulting in $\boldsymbol{t}=\mathbf{I}$. And for the cases of pure rotation, implying that $\boldsymbol{t}=[0,0,0]^{T}$ in Eq. (A.20). 
The 3D pose transformations of the coordinate frames in the Figure above can be expressed in the form of homogeneous transformation matrices if the points $\boldsymbol{p}_{o}$ and $\boldsymbol{p}_{i}$ are in terms of homogeneous coordinates. The transformation matrices

$$
\begin{aligned}
\mathbf{T}_{\text {trans }}\left(t_{x}, t_{y}, t_{z}\right)= & {\left[\begin{array}{cccc}
1 & 0 & 0 & t_{x} \\
0 & 1 & 0 & t_{y} \\
0 & 0 & 1 & t_{z} \\
0 & 0 & 0 & 1
\end{array}\right], \quad \mathbf{T}_{r o t}\left(\mathbf{x}, \theta_{x}\right)=\left[\begin{array}{cccc}
1 & 0 & 0 & 0 \\
0 & c_{\theta_{x}} & -s_{\theta_{x}} & 0 \\
0 & s_{\theta_{x}} & c_{\theta_{x}} & 0 \\
0 & 0 & 0 & 1
\end{array}\right], } \\
\mathbf{T}_{r o t}\left(\mathbf{y}, \theta_{y}\right)= & {\left[\begin{array}{cccc}
c_{\theta_{y}} & 0 & s_{\theta_{y}} & 0 \\
0 & 1 & 0 & 0 \\
-s_{\theta_{y}} & 0 & c_{\theta_{y}} & 0 \\
0 & 0 & 0 & 1
\end{array}\right], \mathbf{T}_{r o t}\left(\mathbf{z}, \theta_{z}\right)=\left[\begin{array}{cccc}
c_{\theta_{z}} & -s_{\theta_{z}} & 0 & 0 \\
s_{\theta_{z}} & c_{\theta_{z}} & 0 & 0 \\
0 & 0 & 1 & 0 \\
0 & 0 & & \\
& & & \\
0 & 0 & 0 & 1
\end{array}\right], }
\end{aligned}
$$

can be used to construct a more complicated transformation (6D pose transformations) through some recursive multiplications.

\section{A.3.2 6D Pose Transformations}

In robot kinematics, computer vision, or computer graphics applications, the transformation between the desired frame $\Sigma_{i}$ and the reference frame $\Sigma_{o}$ are defined with the $6 \mathrm{D}$ pose transformation.

Consider the rotation matrix portion of the homogeneous transformation matrix is expressed in terms of the ZYX rotations (for extracting the roll-pitch-yaw angles), the following sequential transformations can be applied: 


$$
\begin{aligned}
\mathbf{T}_{6 D} & =\mathbf{T}_{\text {trans }}\left(t_{x}, t_{y}, t_{z}\right) \mathbf{T}_{r o t}\left(\mathbf{z}, \theta_{z}\right) \mathbf{T}_{r o t}\left(\mathbf{y}, \theta_{y}\right) \mathbf{T}_{r o t}\left(\mathbf{x}, \theta_{x}\right), \\
& =\left[\begin{array}{cccc}
c_{\theta_{y}} c_{\theta_{z}} & s_{\theta_{x}} s_{\theta_{y}} c_{\theta_{z}}-c_{\theta_{x}} s_{\theta_{z}} & c_{\theta_{x}} s_{\theta_{y}} c_{\theta_{z}}+s_{\theta_{x}} s_{\theta_{z}} & t_{x} \\
c_{\theta_{y}} s_{\theta_{z}} & s_{\theta_{x}} s_{\theta_{y}} s_{\theta_{z}}+c_{\theta_{x}} c_{\theta_{z}} & c_{\theta_{x}} s_{\theta_{y}} s_{\theta_{z}}-s_{\theta_{x}} c_{\theta_{z}} & t_{y} \\
-s_{\theta_{y}} & s_{\theta_{x}} c_{\theta_{y}} & c_{\theta_{x}} c_{\theta_{y}} & t_{z} \\
0 & 0 & 0 & 1
\end{array}\right],
\end{aligned}
$$

where $c_{\gamma}$ and $s_{\gamma}$ represents $\cos \gamma$ and $\sin \gamma$, respectively, and $\gamma$ can be the angles $\theta_{x}$, $\theta_{y}$, or $\theta_{z}$. The sequential transformations made here performed translations to the point first then rotates the frame using ZYX rotation, implying that $\mathbf{t}$ is strictly the location of $\Sigma_{i}$ relative to $\Sigma_{o}$.

\section{A.4 Relative Displacements using Homogeneous Transformation Matrix}

Consider the relative measurements reviewed in Section 2.1, the manipulator is commanded to the configuration $j$ after $i$, which produces the poses, $\boldsymbol{x}_{A, j}$ and $\boldsymbol{x}_{B, j}$. These poses can be expressed in the form of homogeneous transformation matrices,

$$
\begin{array}{rlr}
{ }^{m} \mathbf{T}_{A, i} & =\left[\begin{array}{cc}
{ }^{m} \mathbf{R}_{A, i} & { }^{m} \mathbf{p}_{A, i} \\
\mathbf{0} & 1
\end{array}\right], & { }^{m} \mathbf{T}_{A, j}=\left[\begin{array}{cc}
{ }^{m} \mathbf{R}_{A, j} & { }^{m} \mathbf{p}_{A, j} \\
\mathbf{0} & 1
\end{array}\right], \\
{ }^{b} \mathbf{T}_{B, i}= & {\left[\begin{array}{cc}
{ }^{b} \mathbf{R}_{B, i} & { }^{b} \mathbf{p}_{B, i} \\
\mathbf{0} & 1
\end{array}\right],} & { }^{b} \mathbf{T}_{B, j}=\left[\begin{array}{cc}
{ }^{b} \mathbf{R}_{B, j} & { }^{b} \mathbf{p}_{B, j} \\
\mathbf{0} & 1
\end{array}\right] .
\end{array}
$$


$\mathbf{R}$ is a $3 \times 3$ orthonormal matrix representing the end-effector orientation. $\mathbf{p}$ is a $3 \times 1$ vector representing the end-effector position. The superscript represents the reference frame $\left(\Sigma_{m}\right.$ or $\left.{ }_{b}\right)$ the homogeneous transformation is expressed relative to, and the subscript represents the robot model $(A$ or $B)$ and the pose configuration $(i$ or $j)$.

Using the property of a homogeneous transformation matrix, where the sequential transformations of poses can be expressed as recursive multiplications of homogeneous transformation matrices. Then the pose at configuration $j$ can be obtained by transforming from configuration $i$ as

$$
{ }^{w} \mathbf{T}_{j}={ }^{w} \mathbf{T}_{i}{ }^{i} \mathbf{T}_{j}
$$

The relative poses from $i$ to $j$ for the models, $A$ and $B$, are

$$
\begin{aligned}
& { }^{i} \mathbf{T}_{A, j}=\left({ }^{m} \mathbf{T}_{A, i}\right)^{-1 m} \mathbf{T}_{A, j}=\left[\begin{array}{cc}
{ }^{m} \mathbf{R}_{A, i}{ }^{T} & -{ }^{m} \mathbf{R}_{A, i}{ }^{T m} \mathbf{p}_{A, i} \\
\mathbf{0} & 1
\end{array}\right]\left[\begin{array}{cc}
{ }^{m} \mathbf{R}_{A, j} & { }^{m} \mathbf{p}_{A, j} \\
\mathbf{0} & 1
\end{array}\right] \\
& =\left[\begin{array}{cc}
{ }^{i} \mathbf{R}_{A, j} & { }^{m} \mathbf{R}_{A, i}{ }^{T}\left({ }^{m} \mathbf{p}_{A, j}-{ }^{m} \mathbf{p}_{A, i}\right) \\
\mathbf{0} & 1
\end{array}\right] \text {, } \\
& { }^{i} \mathbf{T}_{B, j}=\left({ }^{b} \mathbf{T}_{B, i}\right)^{-1 b} \mathbf{T}_{B, j}=\left[\begin{array}{cc}
{ }^{b} \mathbf{R}_{B, i}{ }^{T} & -{ }^{b} \mathbf{R}_{B, i}{ }^{T}{ }^{T} \mathbf{p}_{B, i} \\
\mathbf{0} & 1
\end{array}\right]\left[\begin{array}{cc}
{ }^{b} \mathbf{R}_{B, j} & { }^{b} \mathbf{p}_{B, j} \\
\mathbf{0} & 1
\end{array}\right] \\
& =\left[\begin{array}{cc}
{ }^{i} \mathbf{R}_{B, j} & { }^{b} \mathbf{R}_{B, i}{ }^{T}\left({ }^{b} \mathbf{p}_{B, j}-{ }^{b} \mathbf{p}_{B, i}\right) \\
\mathbf{0} & 1
\end{array}\right] \text {. }
\end{aligned}
$$




\section{Appendix B}

\section{Links Velocities Representations}

\section{B.1 Derivative of a Rotation Matrix}

Consider a time-varying rotation matric $\boldsymbol{R}=\boldsymbol{R}(t)$. The orthogonality of $\boldsymbol{R}$, allow the property $\boldsymbol{R}^{-1}=\boldsymbol{R}^{T}$ to be true. Then,

$$
\boldsymbol{R}(t) \boldsymbol{R}^{T}(t)=\mathbf{I}
$$

By differentiating the above condition with respect to time, we get

$$
\dot{\boldsymbol{R}}(t) \boldsymbol{R}^{T}(t)+\boldsymbol{R}(t) \dot{\boldsymbol{R}}^{T}(t)=\boldsymbol{O} .
$$

Let's define another property to simplify the derivation. Let

$$
\tilde{\boldsymbol{\omega}}=\dot{\boldsymbol{R}}(t) \boldsymbol{R}^{T}(t)=-\boldsymbol{R}(t) \dot{\boldsymbol{R}}^{T}(t)=-\left(\dot{\boldsymbol{R}}(t) \boldsymbol{R}^{T}(t)\right)^{T},
$$

where $\tilde{\boldsymbol{\omega}}$ is a $3 \times 3$ skew-symmetric matrix, because Eq. (B.2) becomes

$$
\tilde{\boldsymbol{\omega}}+\tilde{\boldsymbol{\omega}}^{T}=\tilde{\boldsymbol{\omega}}-\tilde{\boldsymbol{\omega}}=\mathbf{0} .
$$


Therefore, by rearranging Eq. (B.3), the time derivative of the rotation matrix becomes

$$
\dot{\boldsymbol{R}}(t)=\tilde{\boldsymbol{\omega}}(t) \boldsymbol{R}(t)
$$

The skew symmetric matrix has the form of

$$
\tilde{\boldsymbol{\omega}}=\left[\begin{array}{ccc}
0 & -\omega_{z} & \omega_{y} \\
\omega_{z} & 0 & -\omega_{x} \\
-\omega_{y} & \omega_{x} & 0
\end{array}\right],
$$

which allows us to express it in a compact form (vector) of the instantaneous angular velocity as $\overrightarrow{\boldsymbol{\omega}}=\left[\omega_{x}, \omega_{y}, \omega_{z}\right]^{T}$.

\section{B.2 Link Velocities}

Consider a generic Link $i$ that is setup from Link $i-1$ as illustrated in Figure B.1. Using the Cartesian form as reviewed for Eq. (A.1), the position vector ${ }^{b} \boldsymbol{p}_{i}$ is

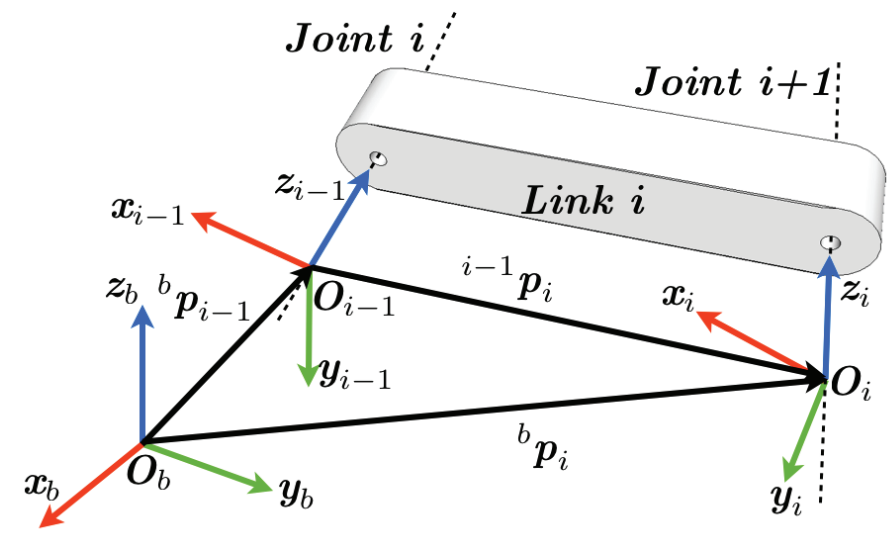

Figure B.1: Pose representation of generic link $i$ of a manipulator. 


$$
{ }^{b} \boldsymbol{p}_{i}={ }^{b} \boldsymbol{p}_{i-1}+{ }^{b} \boldsymbol{R}_{i-1}{ }^{i-1} \boldsymbol{p}_{i}
$$

Because every components are time-variant, differentiating Eq. (B.7) with respect to time gives

$$
\begin{aligned}
{ }^{b} \dot{\boldsymbol{p}}_{i} & ={ }^{b} \dot{\boldsymbol{p}}_{i-1}+{ }^{b} \boldsymbol{R}_{i-1}{ }^{i-1} \dot{\boldsymbol{p}}_{i}+{ }^{b} \dot{\boldsymbol{R}}_{i-1}{ }^{i-1} \boldsymbol{p}_{i} \\
& ={ }^{b} \dot{\boldsymbol{p}}_{i-1}+{ }^{b} \boldsymbol{R}_{i-1}{ }^{i-1} \dot{\boldsymbol{p}}_{i}+{ }^{b} \tilde{\boldsymbol{\omega}}_{i-1}{ }^{b} \boldsymbol{R}_{i-1}{ }^{i-1} \boldsymbol{p}_{i} \\
& ={ }^{b} \dot{\boldsymbol{p}}_{i-1}+{ }^{b} \boldsymbol{R}_{i-1}{ }^{i-1} \dot{\boldsymbol{p}}_{i}+{ }^{b} \overrightarrow{\boldsymbol{\omega}}_{i-1} \times{ }^{b} \boldsymbol{R}_{i-1}{ }^{i-1} \boldsymbol{p}_{i}
\end{aligned}
$$

Now, consider the rotation from Frame $i-1$ to $i$, the time-varying rotational matrix is

$$
{ }^{b} \boldsymbol{R}_{i}={ }^{b} \boldsymbol{R}_{i-1}{ }^{i-1} \boldsymbol{R}_{i}
$$

and its time derivative is

$$
\begin{aligned}
{ }^{b} \dot{\boldsymbol{R}}_{i}={ }^{b} \tilde{\boldsymbol{\omega}}_{i}{ }^{b} \boldsymbol{R}_{i} & ={ }^{b} \dot{\boldsymbol{R}}_{i-1}{ }^{i-1} \boldsymbol{R}_{i}+{ }^{b} \boldsymbol{R}_{i-1}{ }^{i-1} \dot{\boldsymbol{R}}_{i}, \\
& ={ }^{b} \tilde{\boldsymbol{\omega}}_{i-1}{ }^{b} \boldsymbol{R}_{i-1}{ }^{i-1} \boldsymbol{R}_{i}+{ }^{b} \boldsymbol{R}_{i-1}{ }^{i-1} \tilde{\boldsymbol{\omega}}_{i-1, i}{ }^{i-1} \boldsymbol{R}_{i}, \\
& ={ }^{b} \tilde{\boldsymbol{\omega}}_{i-1}{ }^{b} \boldsymbol{R}_{i}+{ }^{b} \boldsymbol{R}_{i-1}{ }^{i-1} \tilde{\boldsymbol{\omega}}_{i-1, i}{ }^{b} \boldsymbol{R}_{i-1}^{T}{ }^{b} \boldsymbol{R}_{i-1}{ }^{i-1} \boldsymbol{R}_{i},
\end{aligned}
$$

with a little rearranging, the skew-symmetric matrix ${ }^{b} \tilde{\boldsymbol{\omega}}_{i}$ can be obtained to be

$$
{ }^{b} \tilde{\boldsymbol{\omega}}_{i}={ }^{b} \tilde{\boldsymbol{\omega}}_{i-1}+{ }^{b} \boldsymbol{R}_{i-1}{ }^{i-1} \tilde{\boldsymbol{\omega}}_{i-1, i}{ }^{b} \boldsymbol{R}_{i-1}^{T}
$$

Using the properties of the orthonormality in $\boldsymbol{R}$ and the skew symmetric matrix $\tilde{\boldsymbol{\omega}}$ we can get

$$
{ }^{b} \boldsymbol{R}_{i-1}{ }^{i-1} \tilde{\boldsymbol{\omega}}_{i-1, i}{ }^{b} \boldsymbol{R}_{i-1}^{T}={ }^{b} \boldsymbol{R}_{i-1}{ }^{i-1} \overrightarrow{\boldsymbol{\omega}}_{i-1, i}
$$

Then Eq. (B.11) becomes

$$
{ }^{b} \overrightarrow{\boldsymbol{\omega}}_{i}={ }^{b} \overrightarrow{\boldsymbol{\omega}}_{i-1}+{ }^{b} \boldsymbol{R}_{i-1}{ }^{i-1} \overrightarrow{\boldsymbol{\omega}}_{i-1, i}={ }^{b} \overrightarrow{\boldsymbol{\omega}}_{i-1}+{ }^{b} \overrightarrow{\boldsymbol{\omega}}_{i-1, i}
$$


In general, the angular and linear velocities of Frame $i$ from Frame $i-1$ for prismatic joint (P-pairs) and relative joint (R-pairs) are different. Since this thesis is focused only on R-pairs joint manipulators, the linear velocity is

$$
{ }^{b} \boldsymbol{R}_{i-1}{ }^{i-1} \dot{\boldsymbol{p}}_{i}={ }^{i-1} \overrightarrow{\boldsymbol{\omega}}_{i} \times{ }^{b} \boldsymbol{R}_{i-1}{ }^{i-1} \boldsymbol{p}_{i} .
$$

and the angular velocity is

$$
{ }^{i-1} \overrightarrow{\boldsymbol{\omega}}_{i}=\dot{\theta} \hat{\boldsymbol{k}}_{i-1}
$$

Hence, the angular and linear velocities from Eqs. (B.8) and (B.13), respectively, becomes

$$
\begin{aligned}
{ }^{b} \dot{\boldsymbol{p}}_{i} & ={ }^{b} \dot{\boldsymbol{p}}_{i-1}+{ }^{b} \overrightarrow{\boldsymbol{\omega}}_{i} \times{ }^{b} \boldsymbol{R}_{i-1}{ }^{i-1} \boldsymbol{p}_{i}={ }^{b} \dot{\boldsymbol{p}}_{i-1}+{ }^{b} \boldsymbol{R}_{i-1}\left({ }^{i-1} \overrightarrow{\boldsymbol{\omega}}_{i} \times{ }^{i-1} \boldsymbol{p}_{i}\right), \\
{ }^{b} \overrightarrow{\boldsymbol{\omega}}_{i} & ={ }^{b} \overrightarrow{\boldsymbol{\omega}}_{i-1}+\dot{\theta} \hat{\boldsymbol{k}}_{i-1} .
\end{aligned}
$$

For the linear velocity of the Frame $n$, the time derivative of ${ }^{b} \boldsymbol{p}_{n}$ can be expressed from each contributed linear velocities due to joint $i$ as

$$
\dot{p}_{n}=\sum_{i=1}^{n} \frac{\partial \boldsymbol{p}_{n}}{\partial \theta_{i}} \dot{\theta}_{i}=\sum_{i=1}^{n} \mathcal{J}_{p_{i}} \dot{\theta}_{i}
$$

Observing that the contribution to the linear velocity is to be computed for the Frame $n$, then

$$
\dot{\theta}_{i} \mathcal{J}_{p_{i}}={ }^{i-1} \overrightarrow{\boldsymbol{\omega}}_{i} \times{ }^{i-1} \boldsymbol{p}_{i}=\dot{\theta}_{i} \hat{\boldsymbol{k}}_{i-1} \times\left({ }^{i-1} \boldsymbol{p}_{n}\right) .
$$

For the contribution to the angular velocity on the other hand, it is

$$
\overrightarrow{\boldsymbol{\omega}}_{n}=\sum_{i=1}^{n}{ }^{i-1} \overrightarrow{\boldsymbol{\omega}}_{i}=\sum_{i=1}^{n} \mathcal{J}_{o_{i}} \dot{\theta}_{i}=\sum_{i=1}^{n} \dot{\theta}_{i} \hat{\boldsymbol{k}}_{i-1}
$$




\section{Appendix C}

\section{MATLAB Code for the Experiments}

\section{C.1 The Commonly Used Functions}

rotation_matrix.m

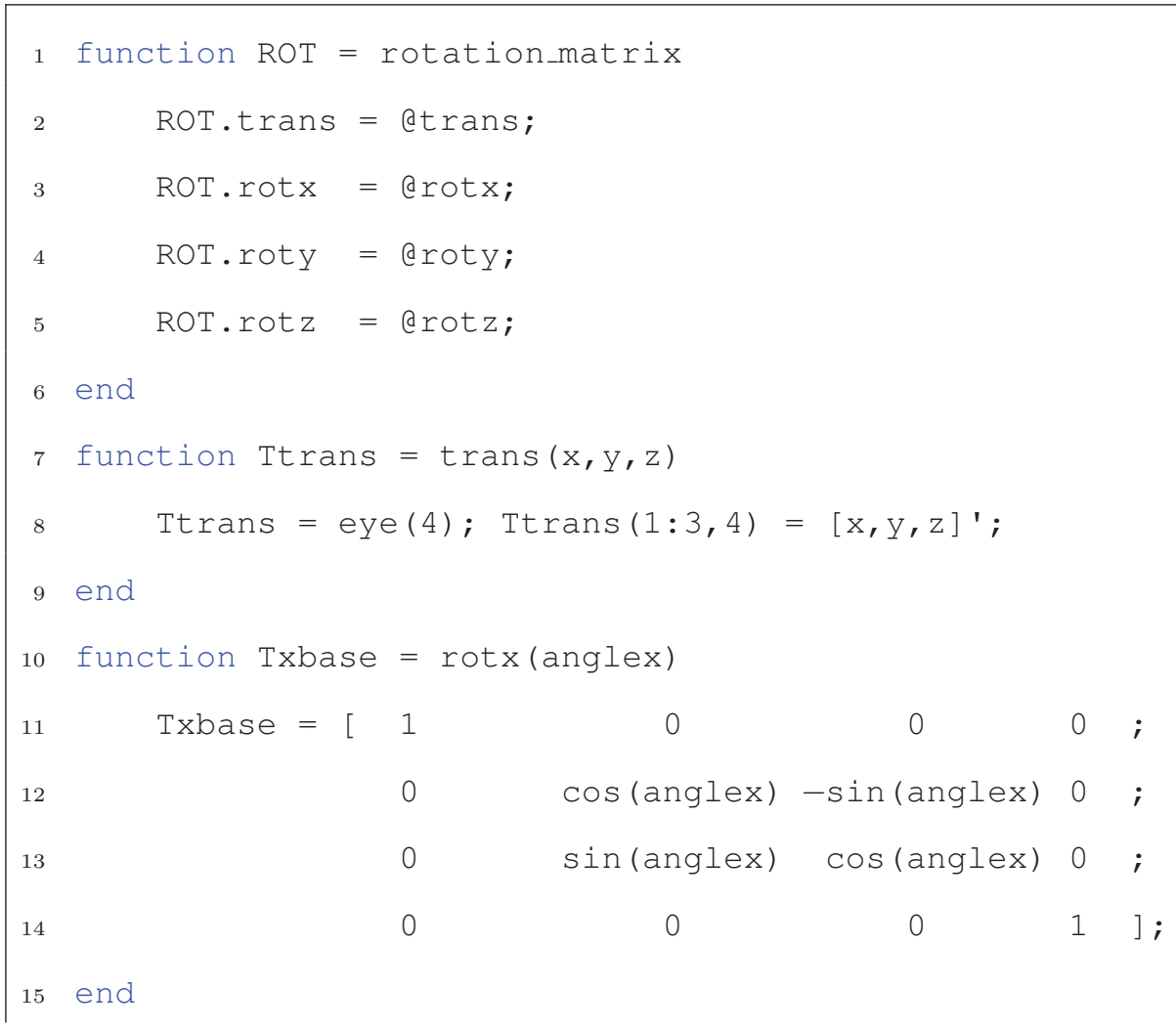




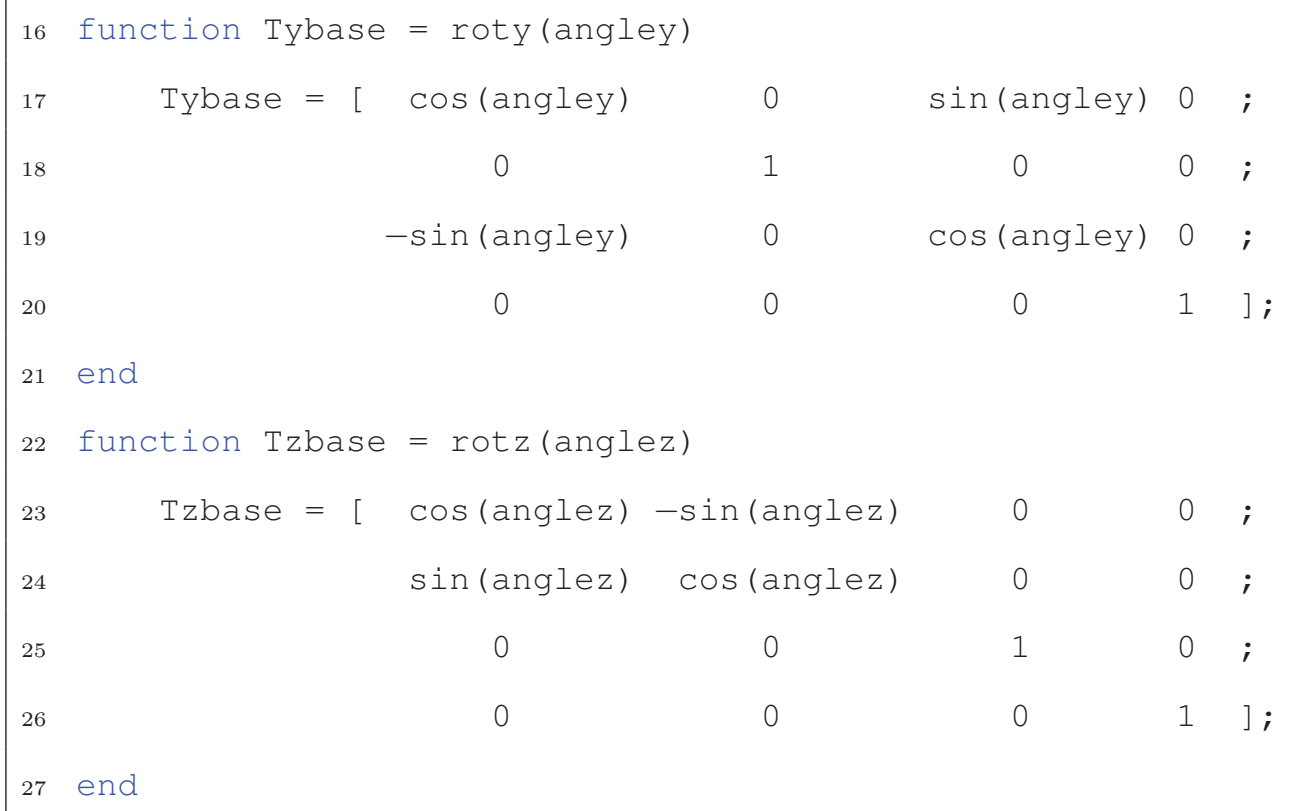

\section{random_noise.m}

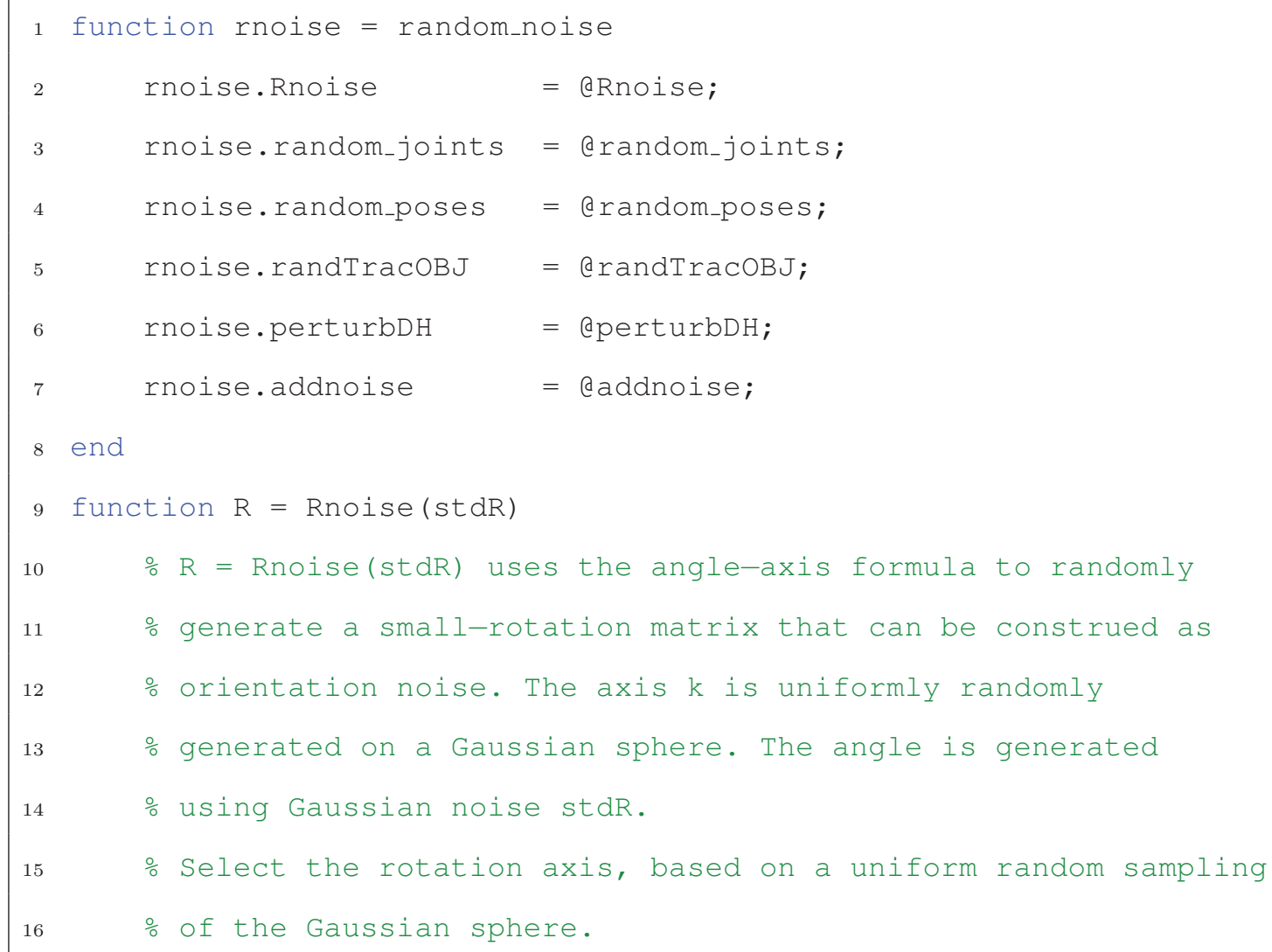




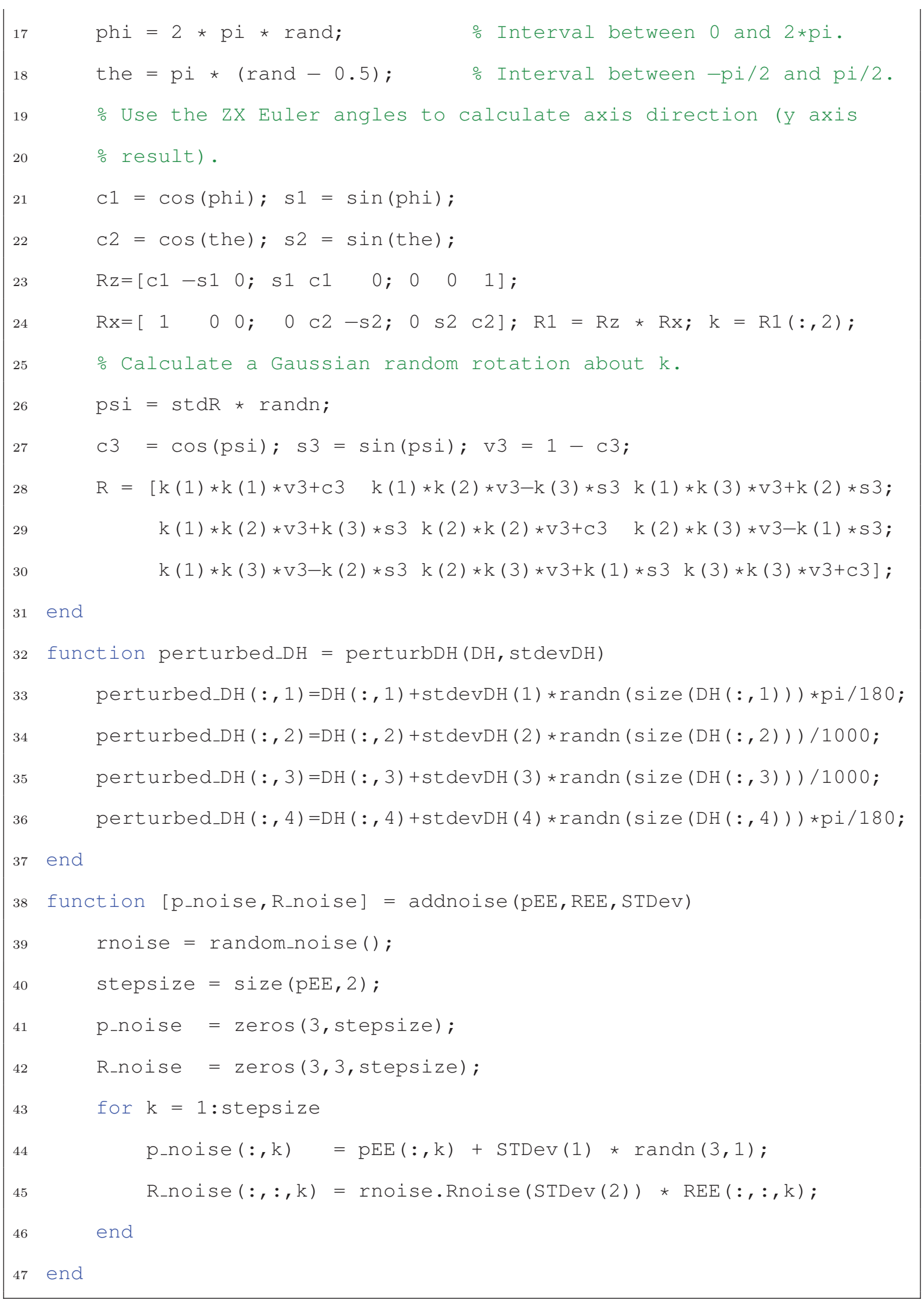




\section{differential_kinematics.m}

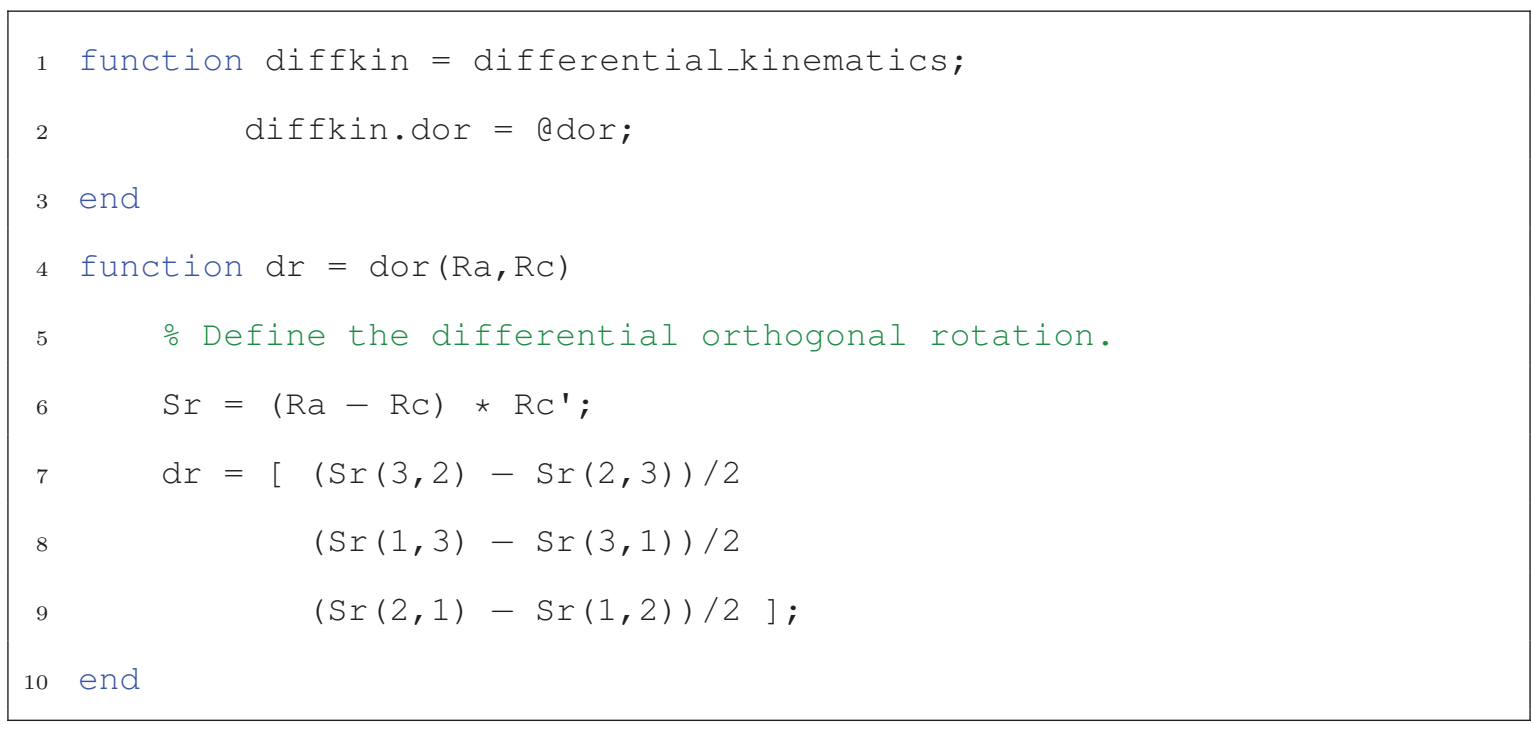

\section{kincal.m}

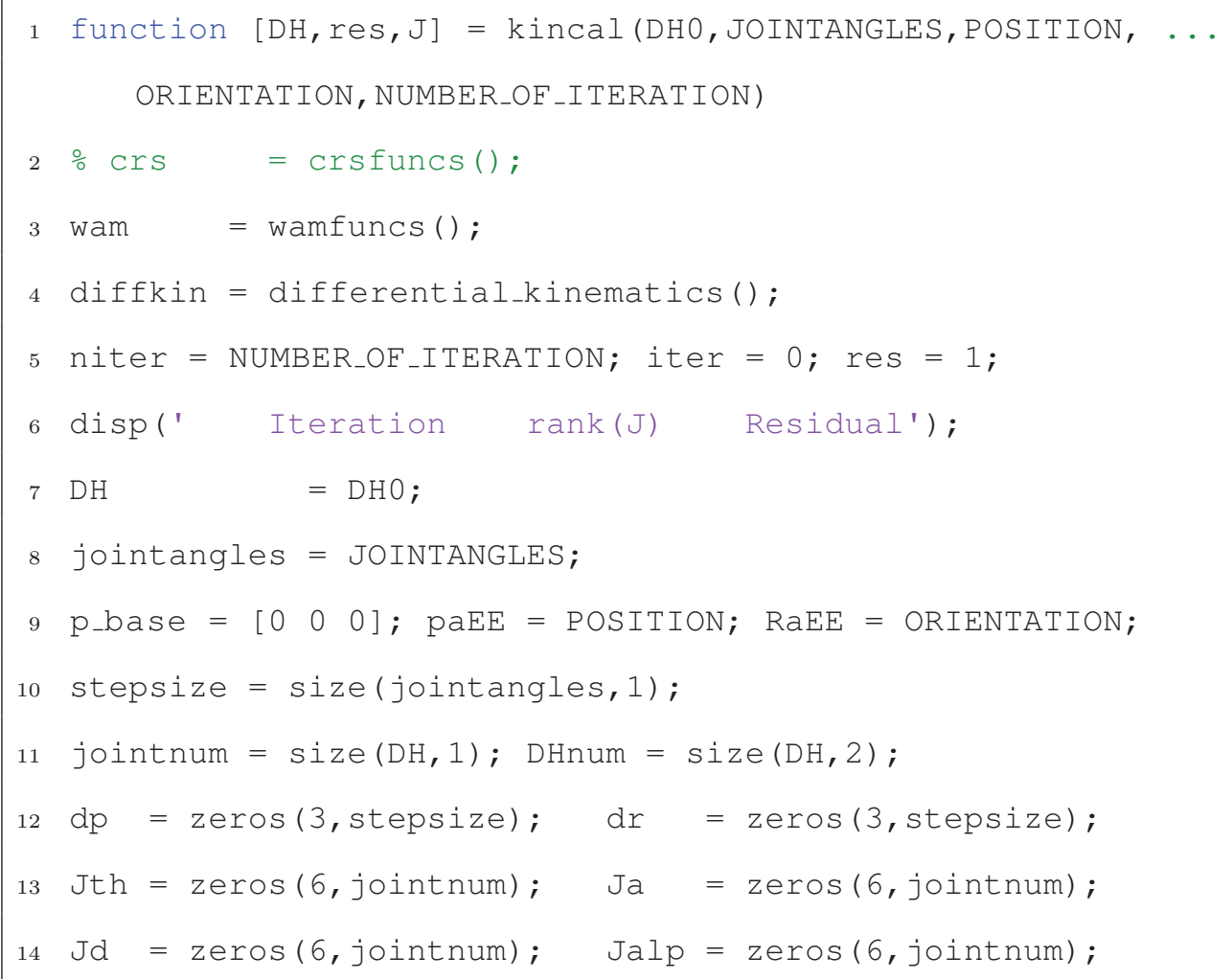




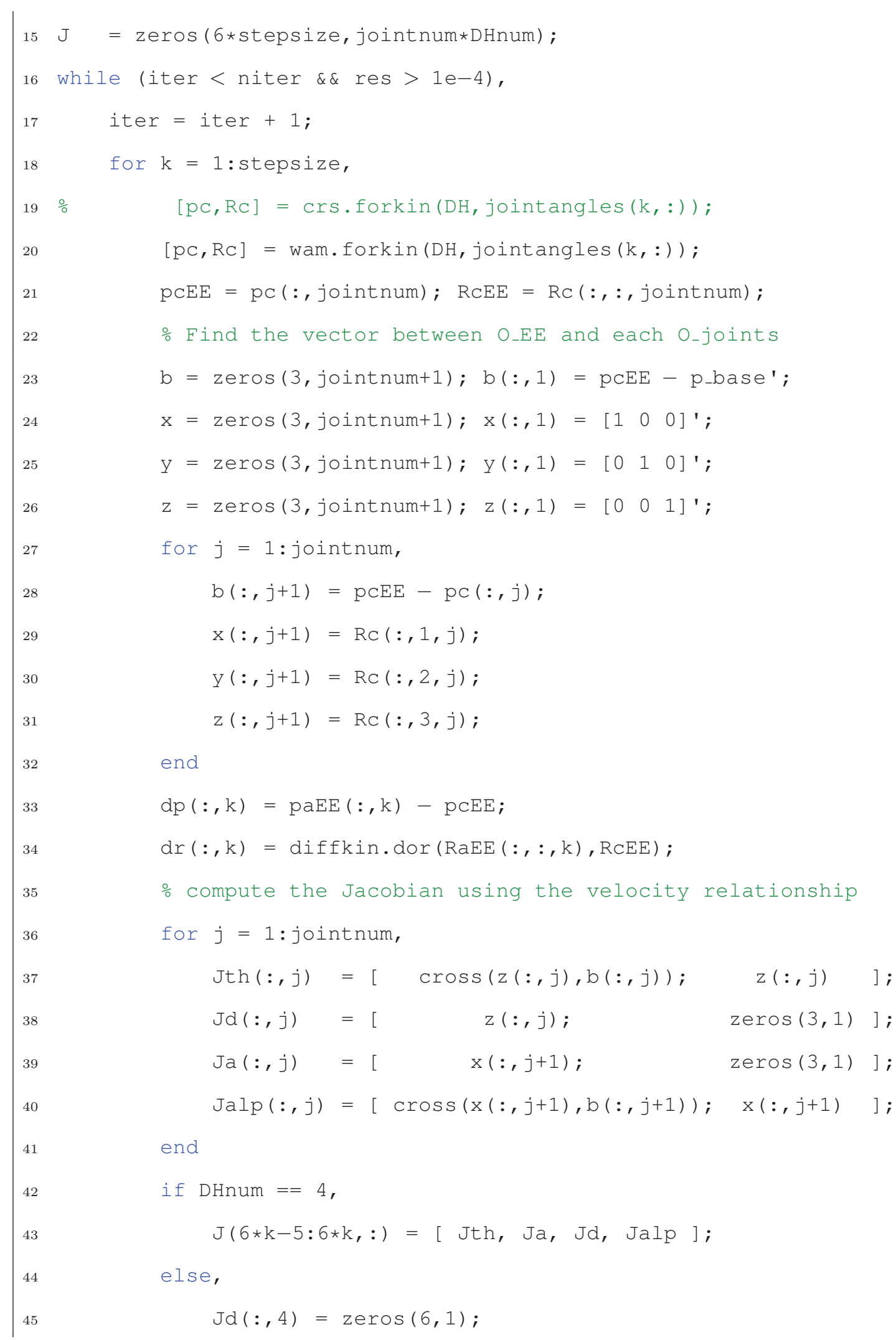




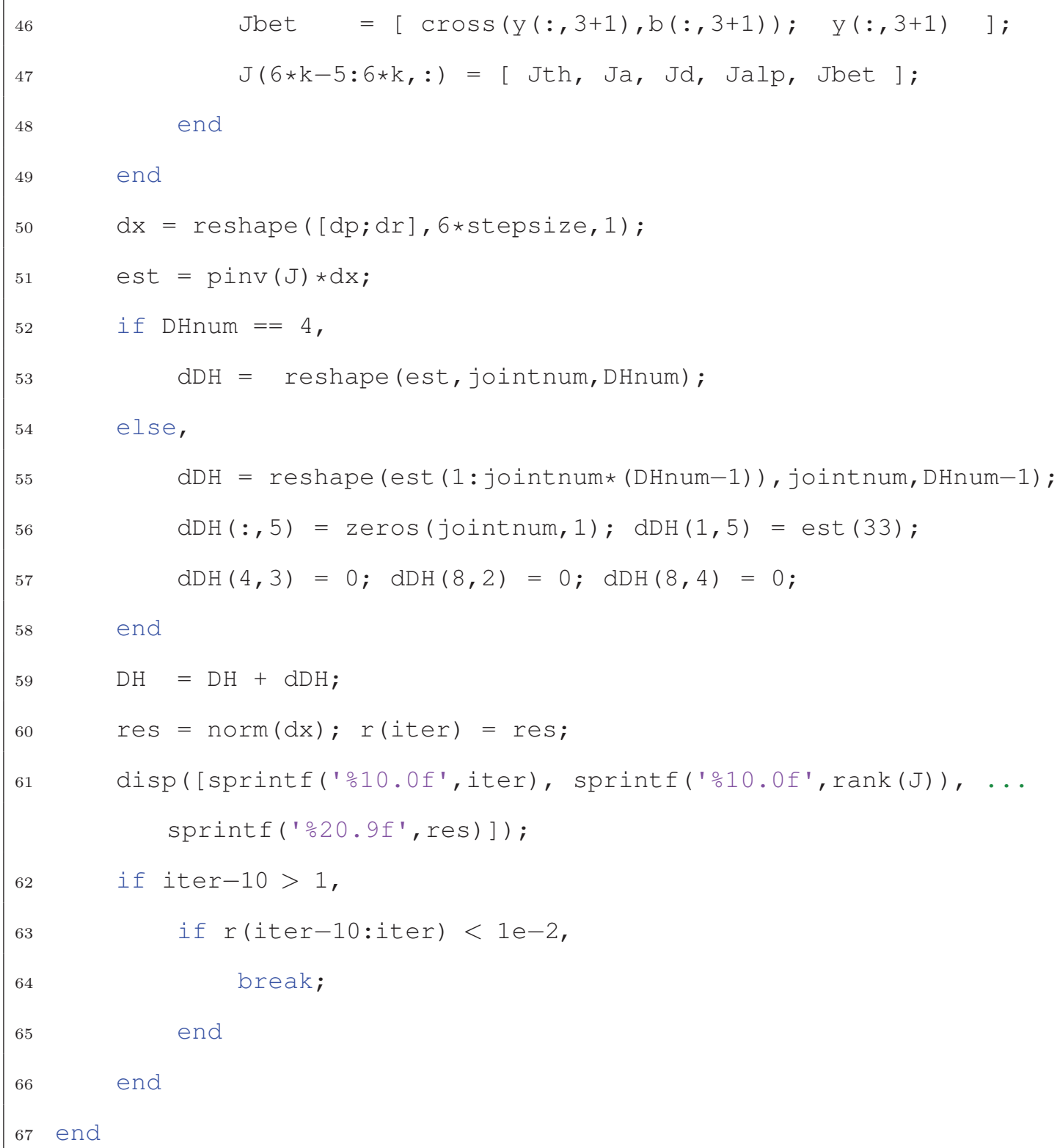

46

Jbet $\quad=[\operatorname{cross}(y(:, 3+1), \mathrm{b}(:, 3+1)) ; \mathrm{y}(:, 3+1)]$; $\mathrm{J}(6 * \mathrm{k}-5: 6 * \mathrm{k},:)=[\mathrm{Jth}, \mathrm{Ja}, \mathrm{Jd}, \mathrm{Jalp}, \mathrm{Jbet}]$; end $\mathrm{dDH}=$ reshape (est, jointnum, DHnum);

\section{kincal_rel.m}

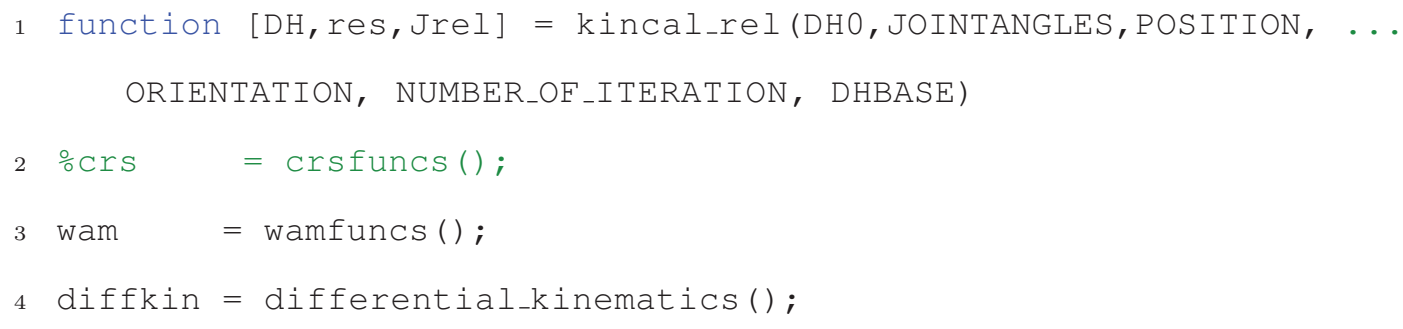




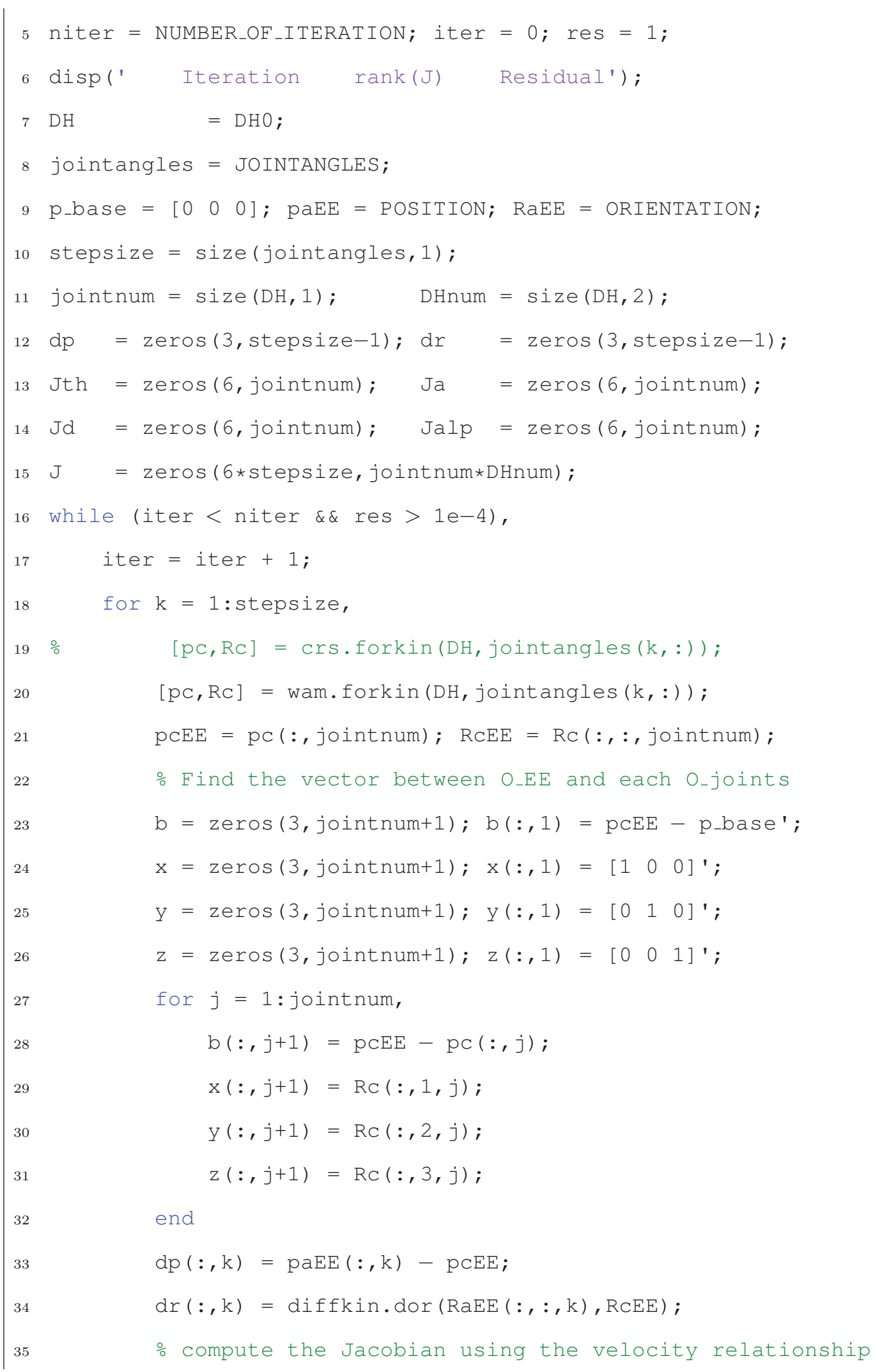




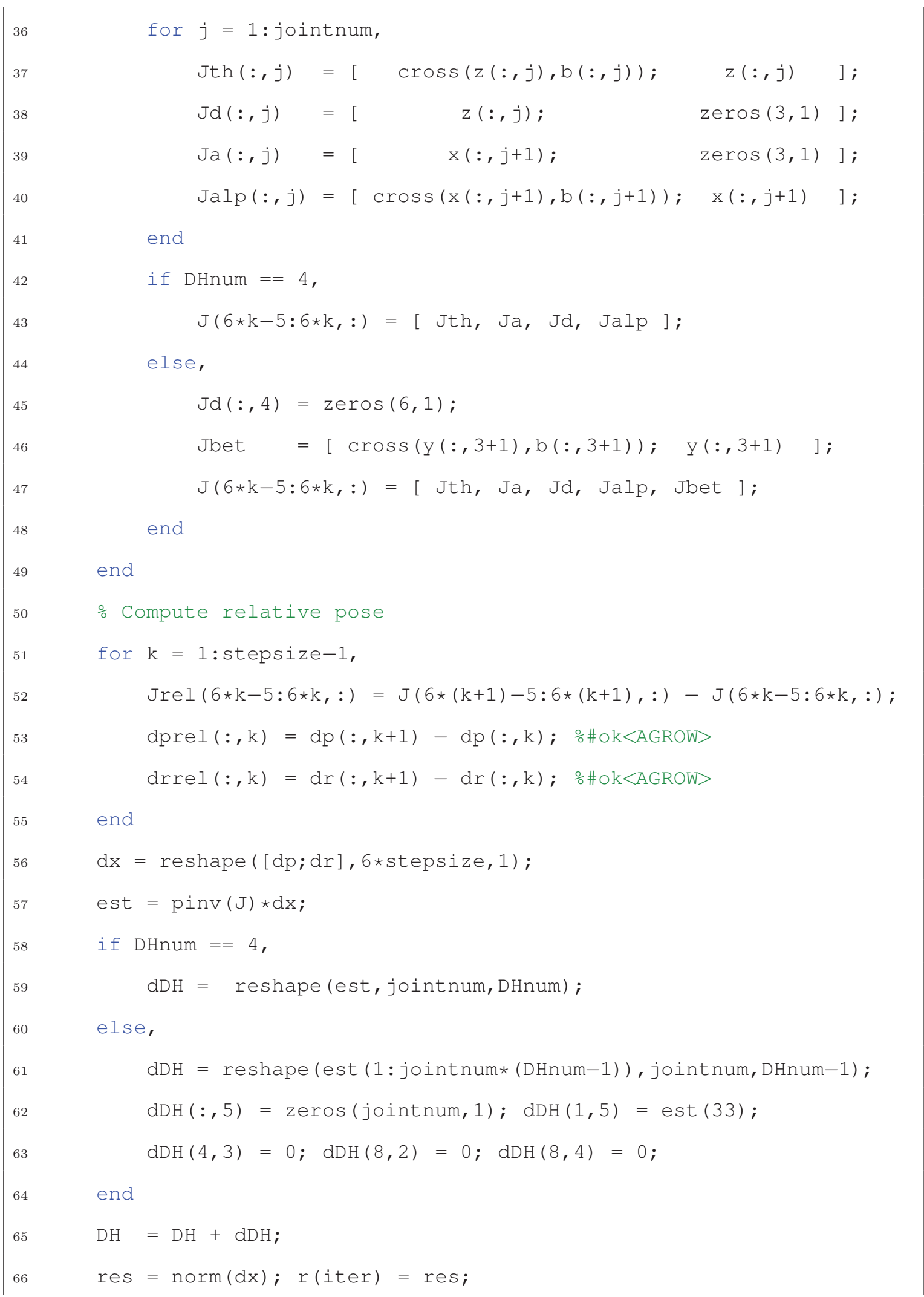


67 disp([sprintf('⒑0f', iter), sprintf('⒑0f', rank(J)), .. sprintf('⒛9f', res)]);

if iter-10>1,

if $r($ iter -10 :iter $)<1 e-2$,

break;

end

72 end

73 end

\section{C.2 m-file Code for the Thermo CRS A465}

\section{crsfuncs.m}

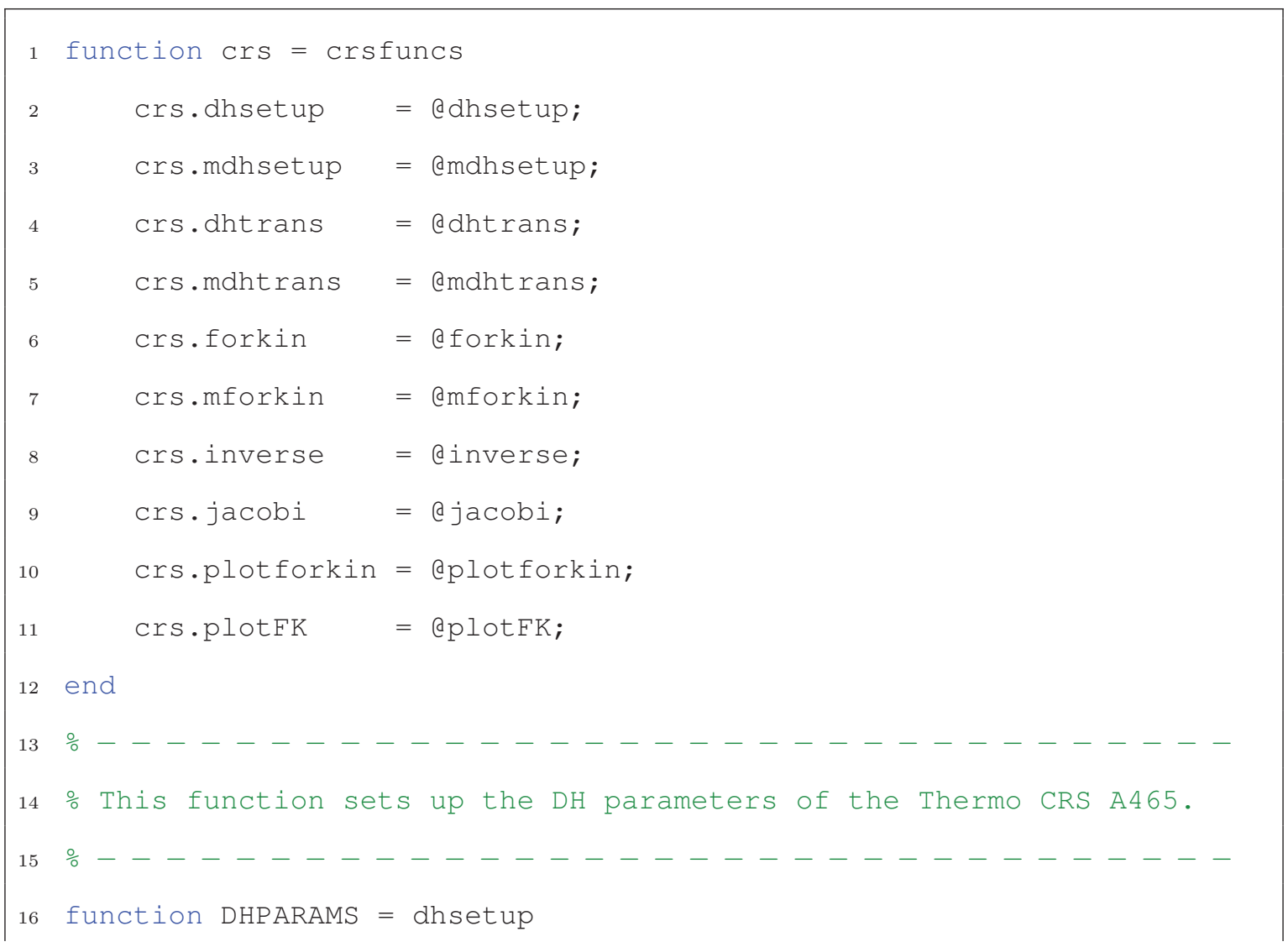




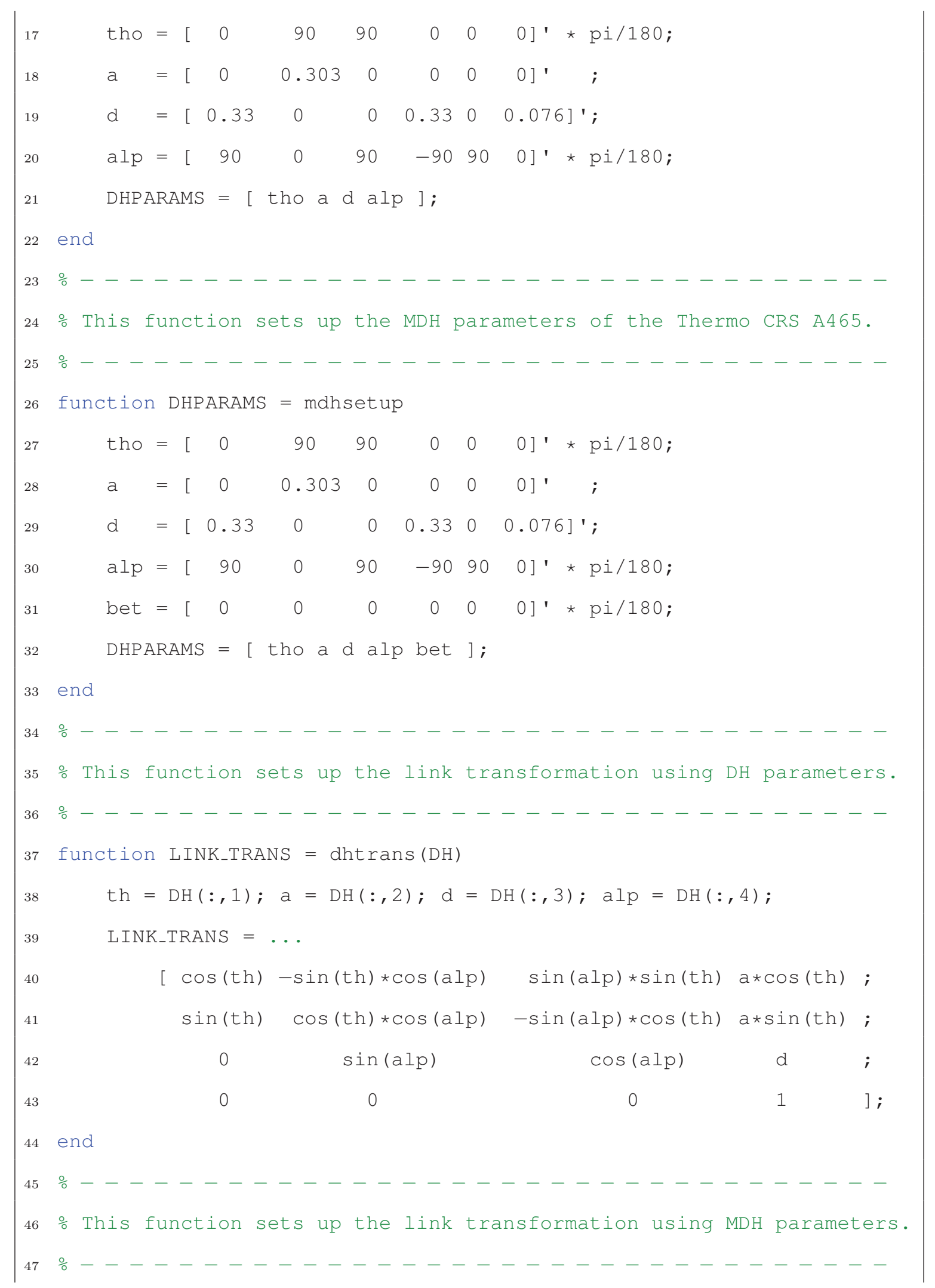




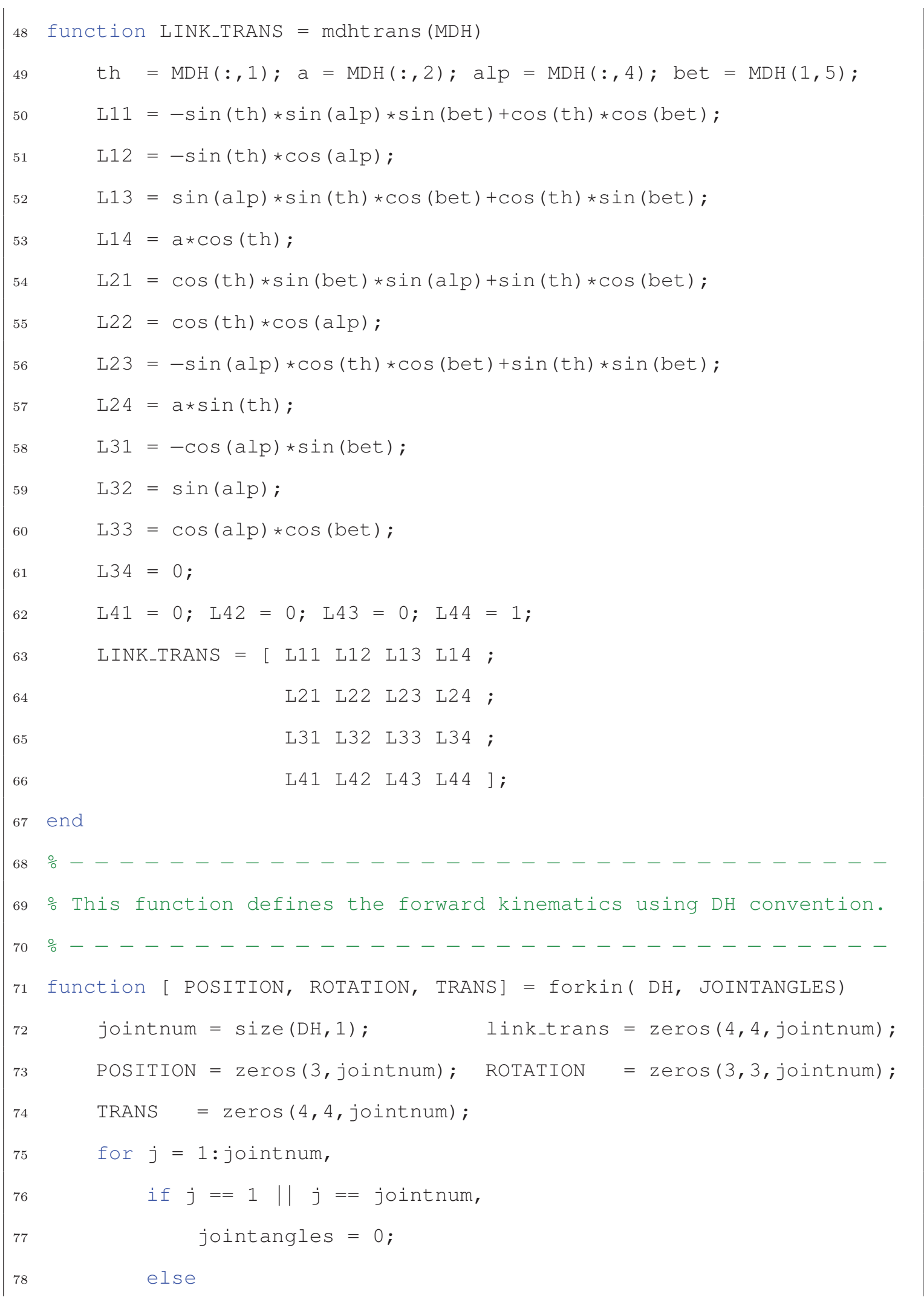




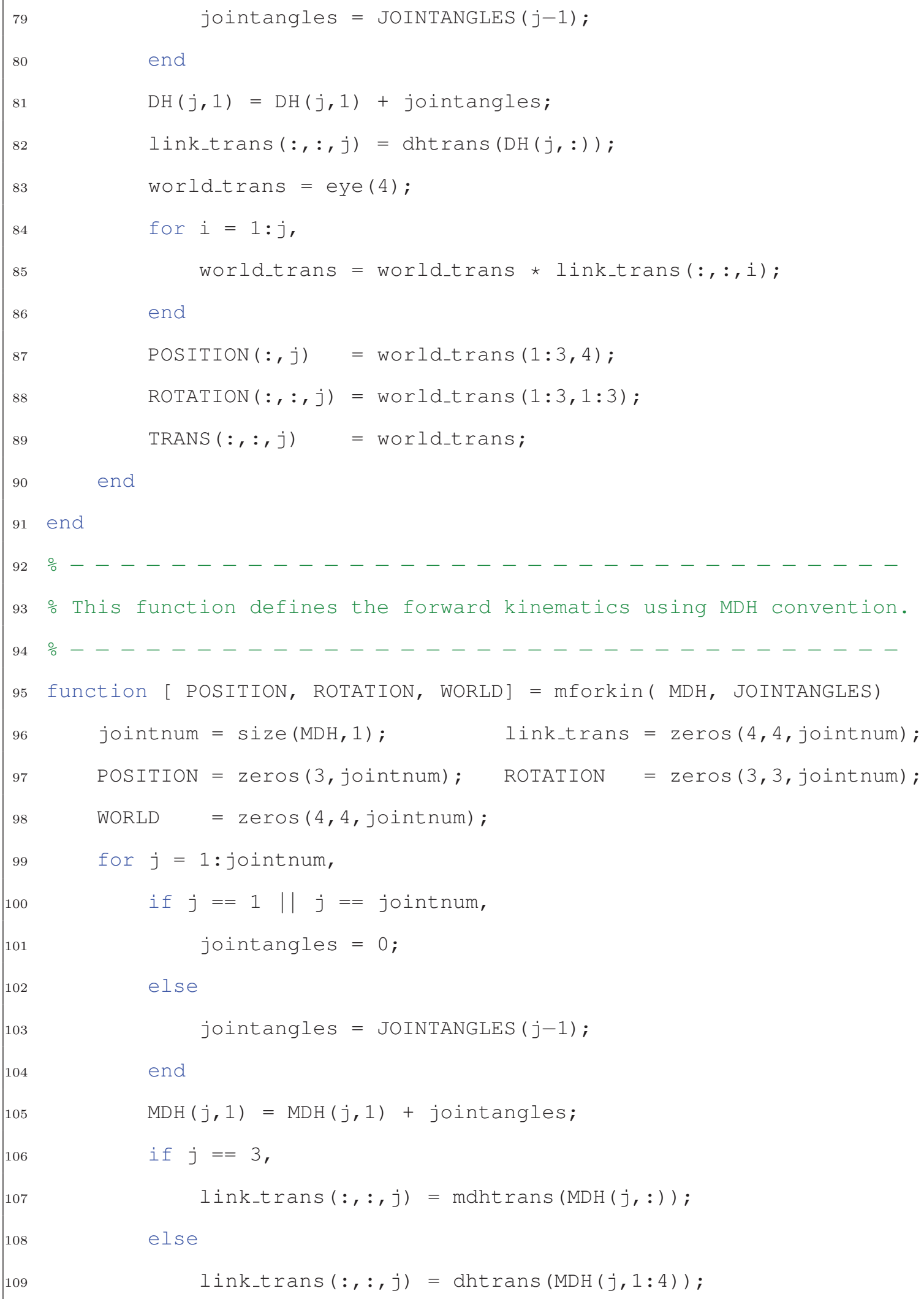




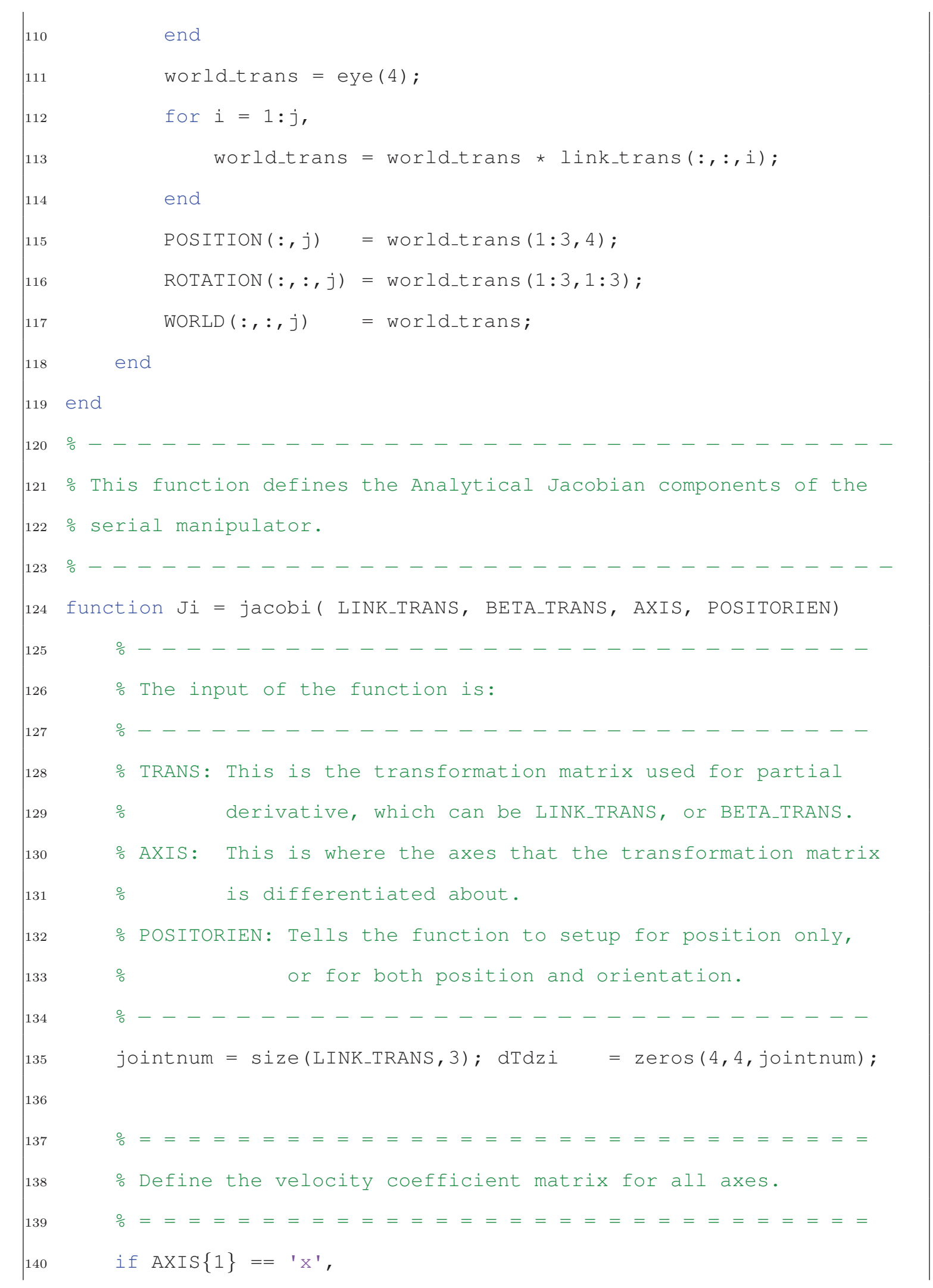




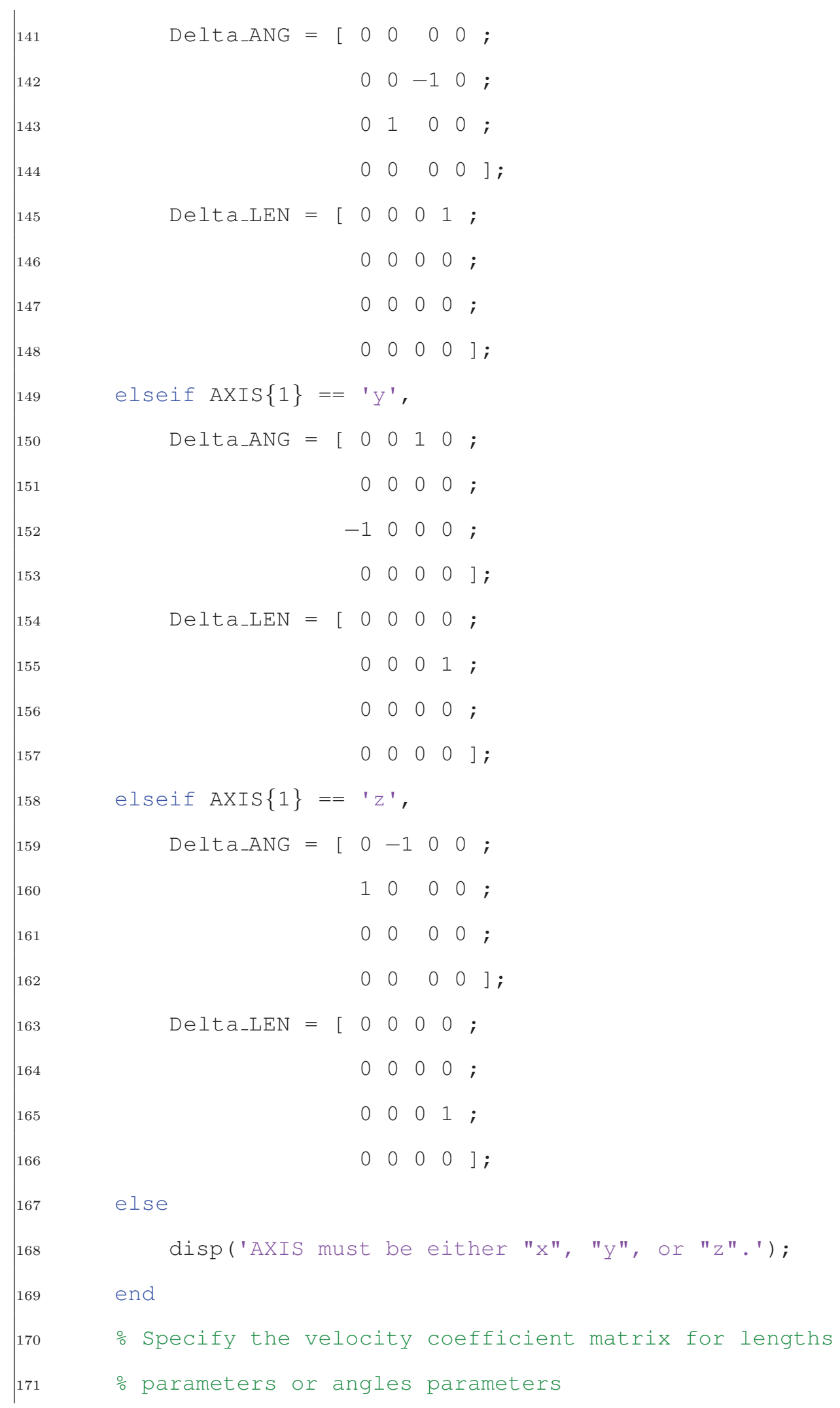




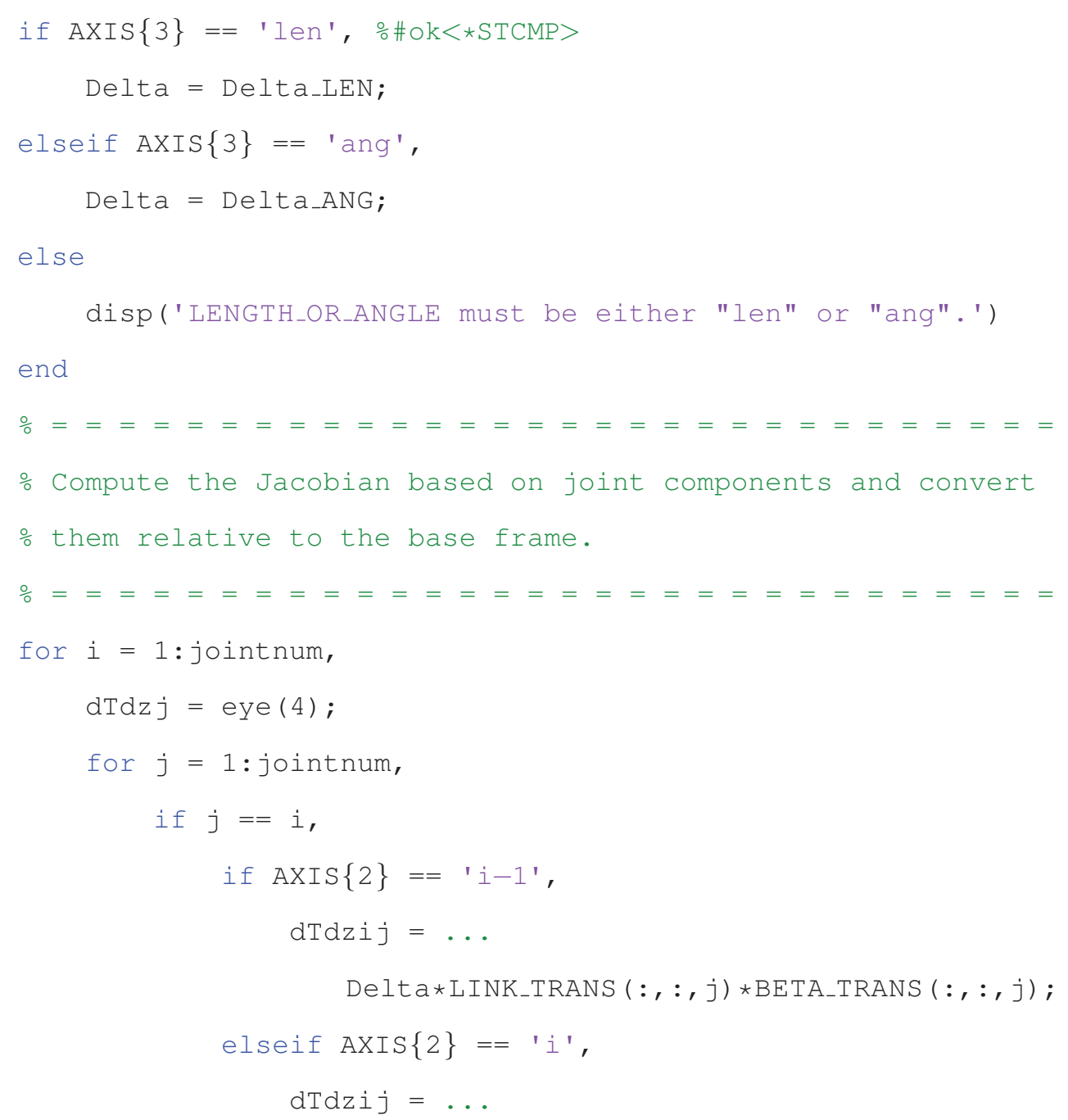




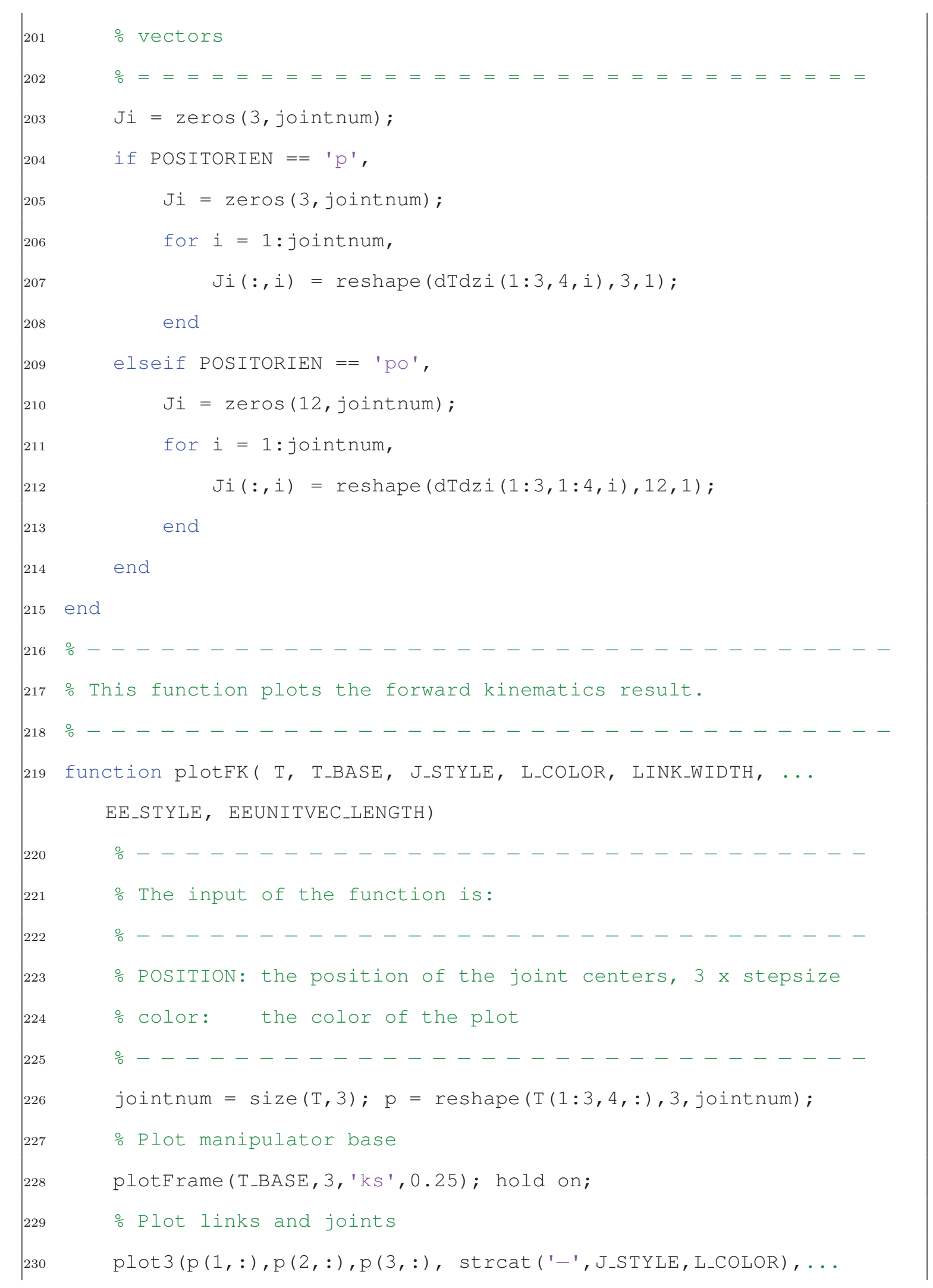




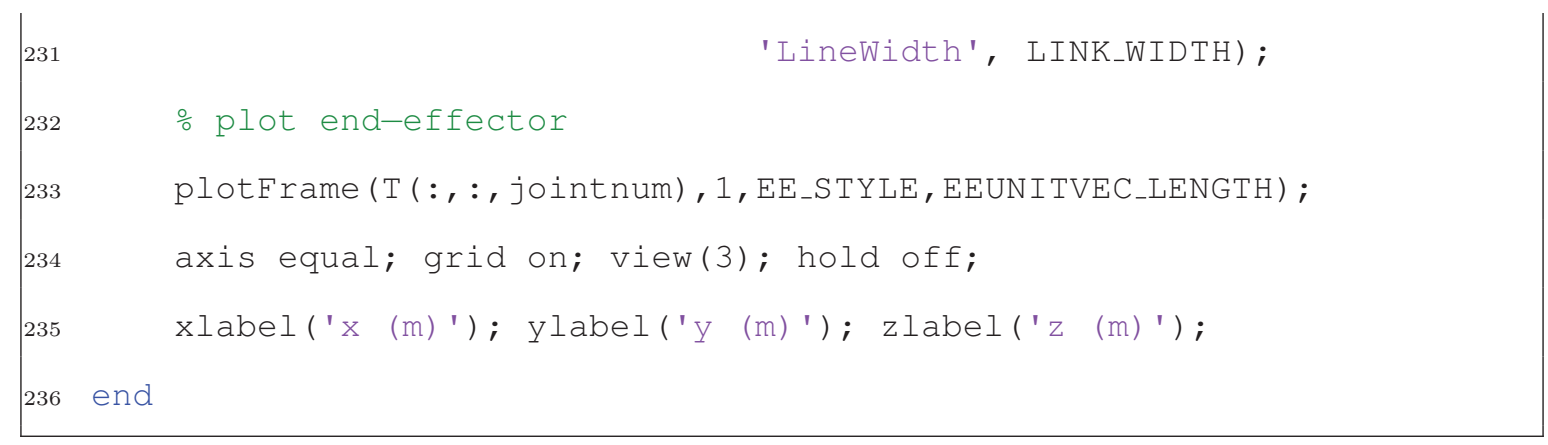

\section{crskincal_main.m}

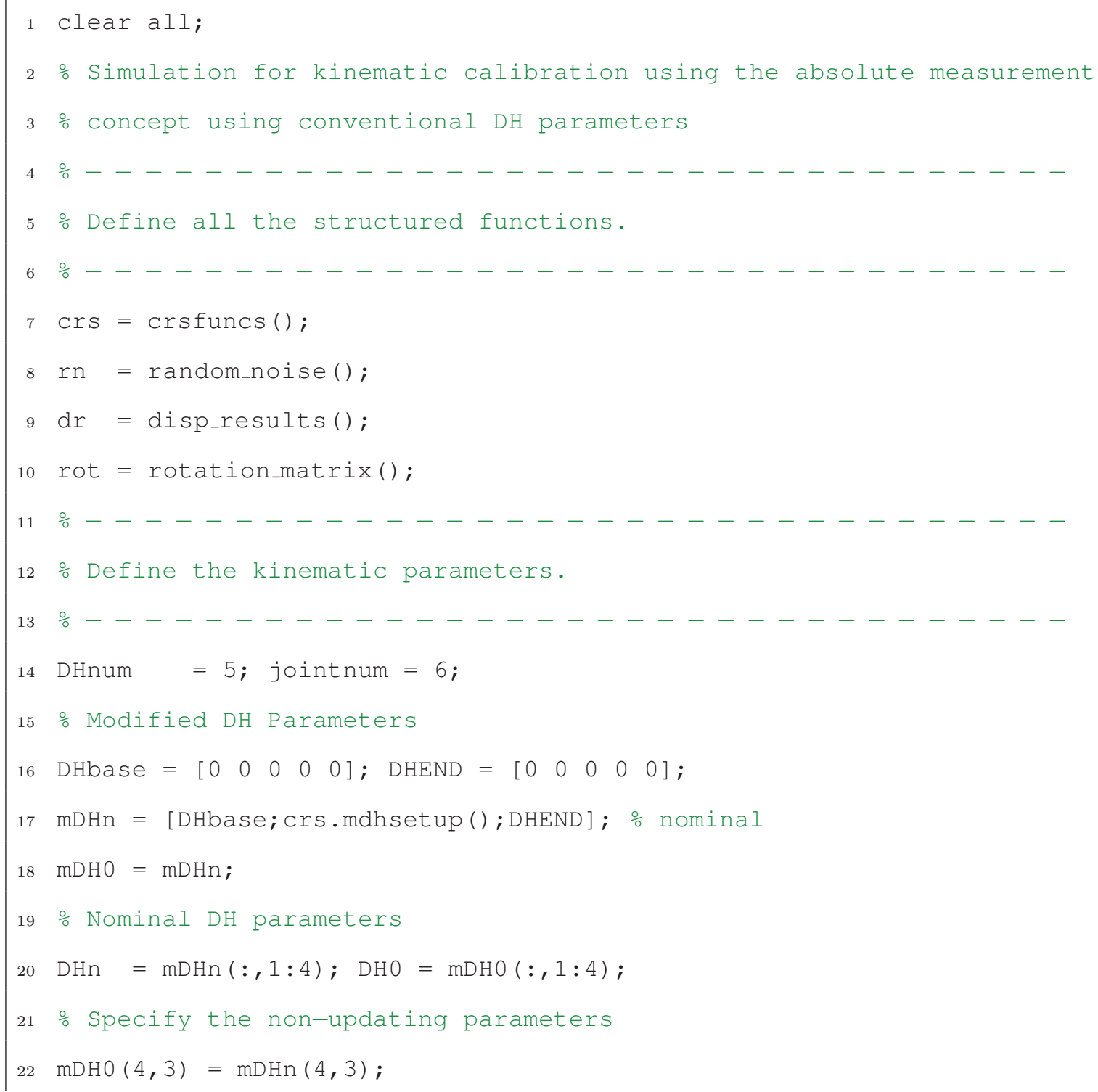




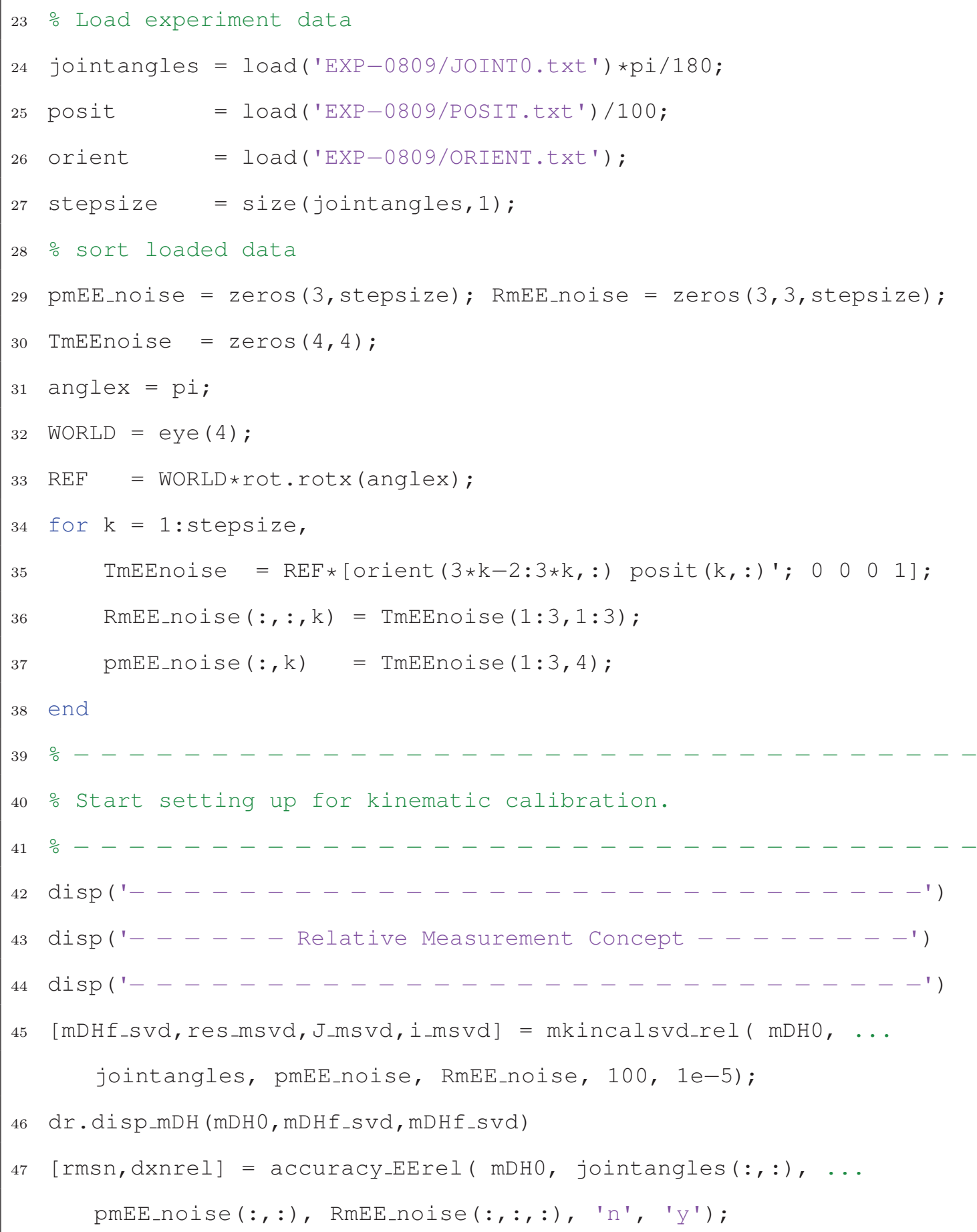




\section{C.3 m-file Code for the 7 DOF WAM}

\section{wamkincal_main.m}

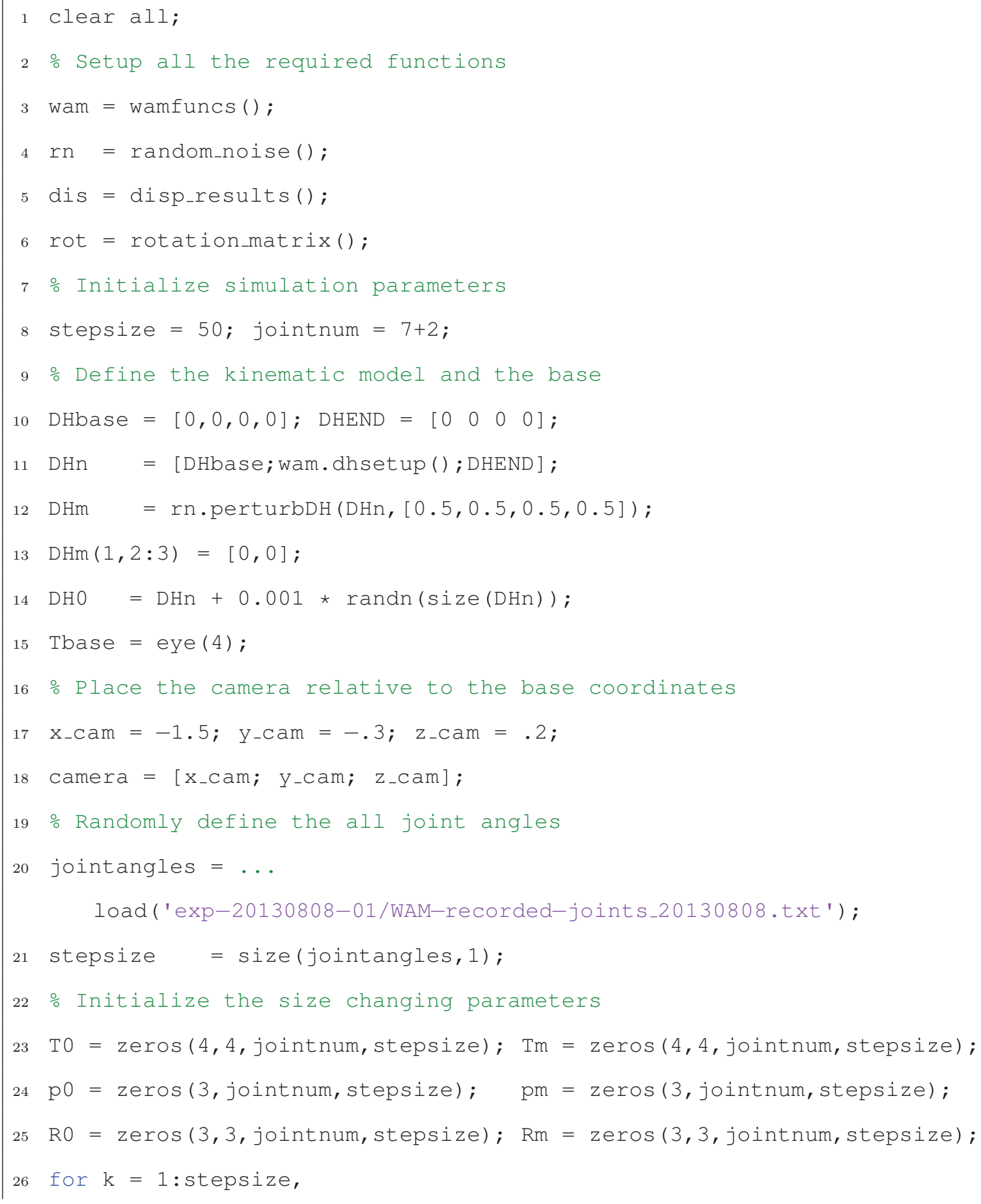




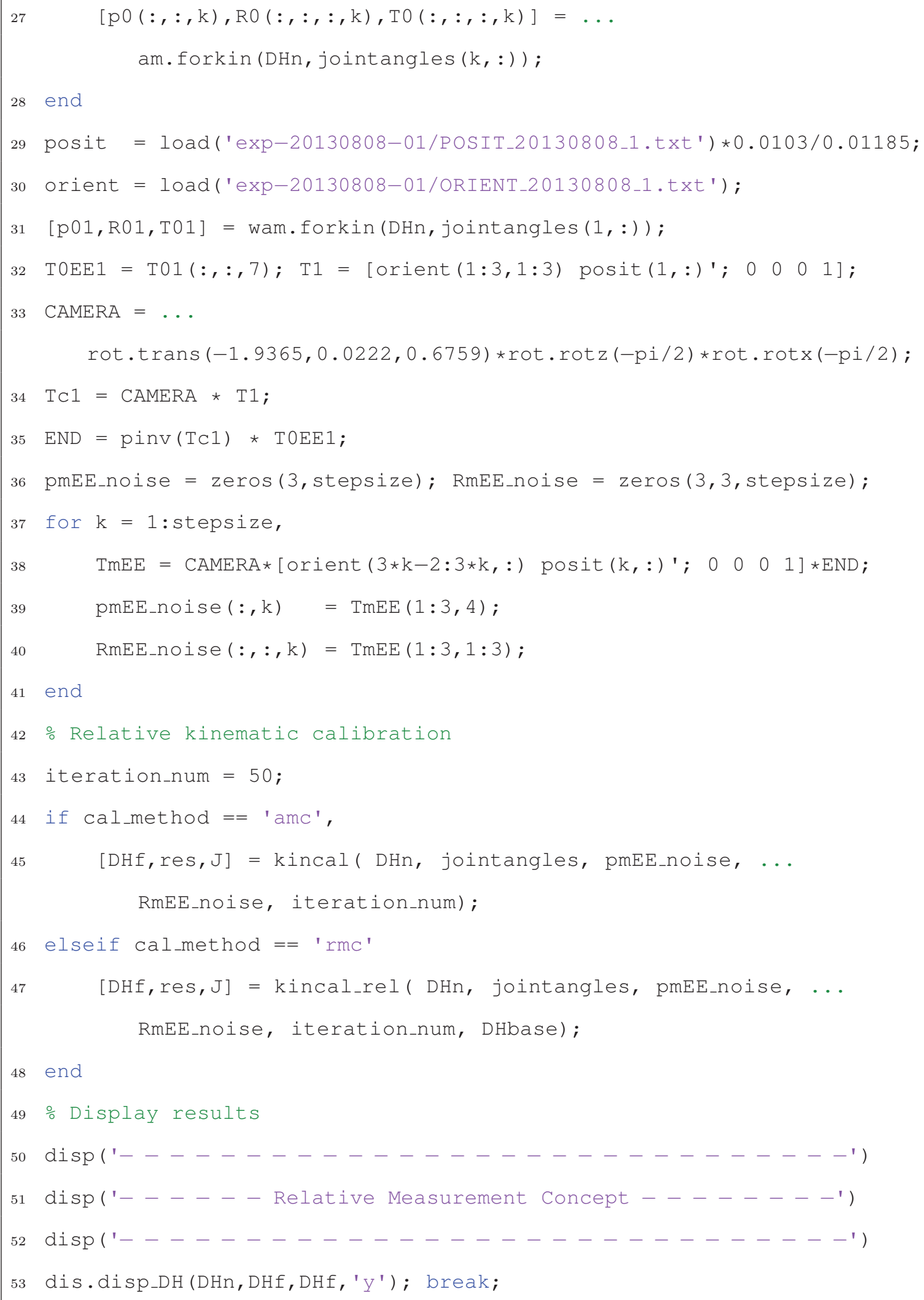




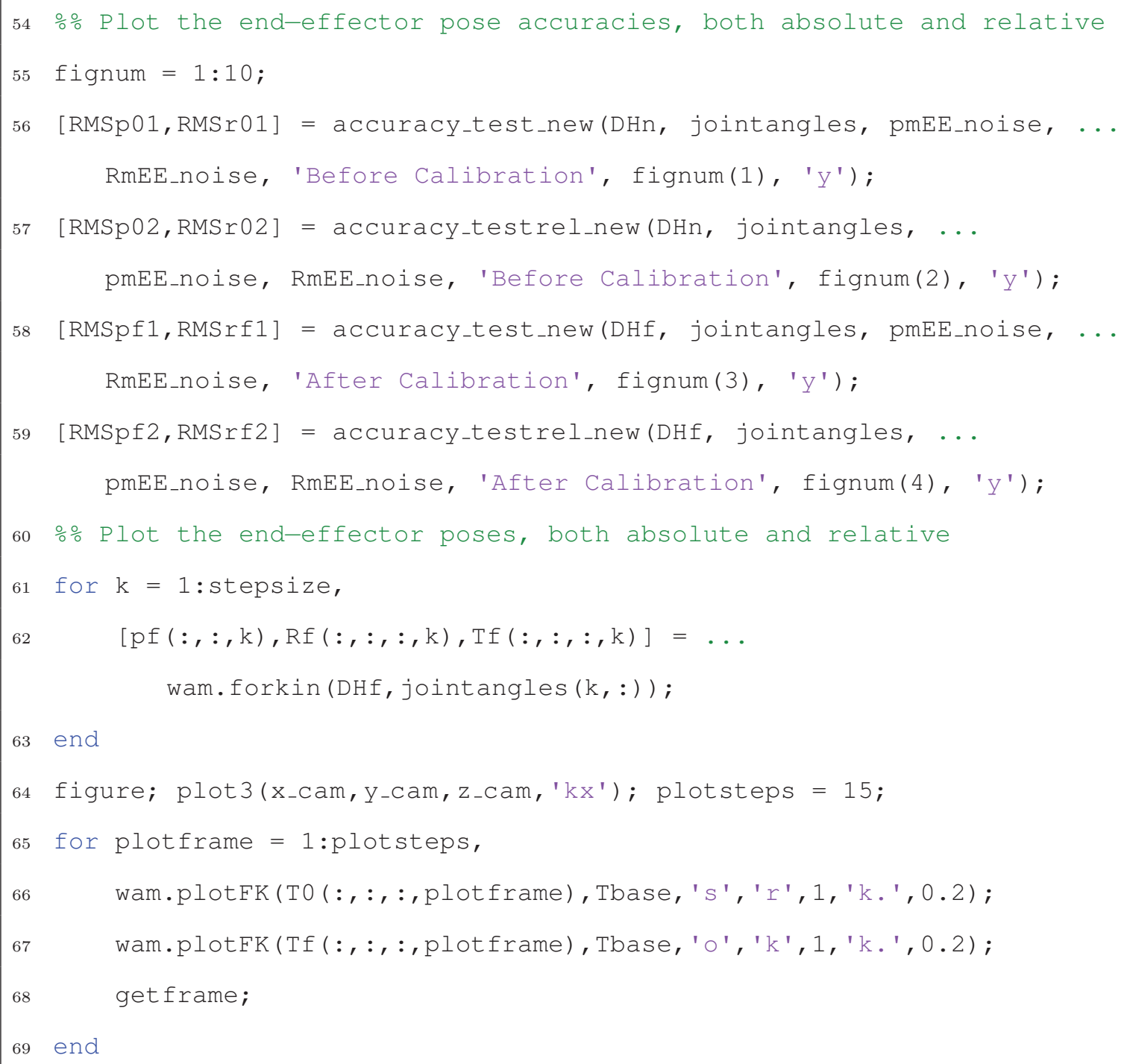

wamfuncs.m

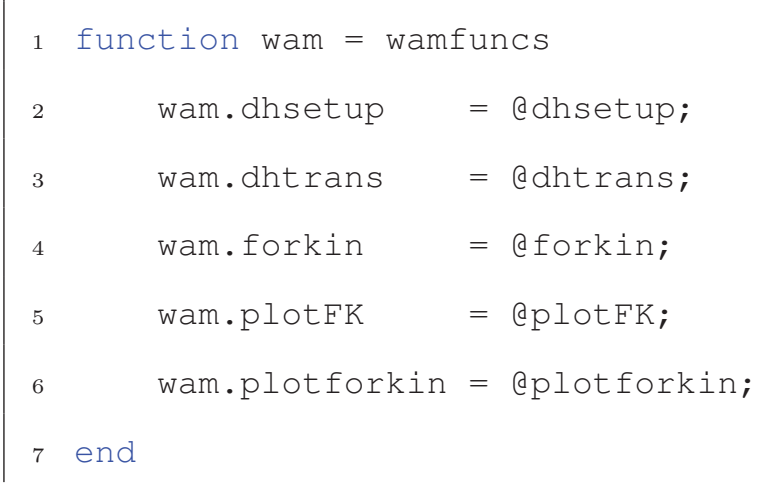




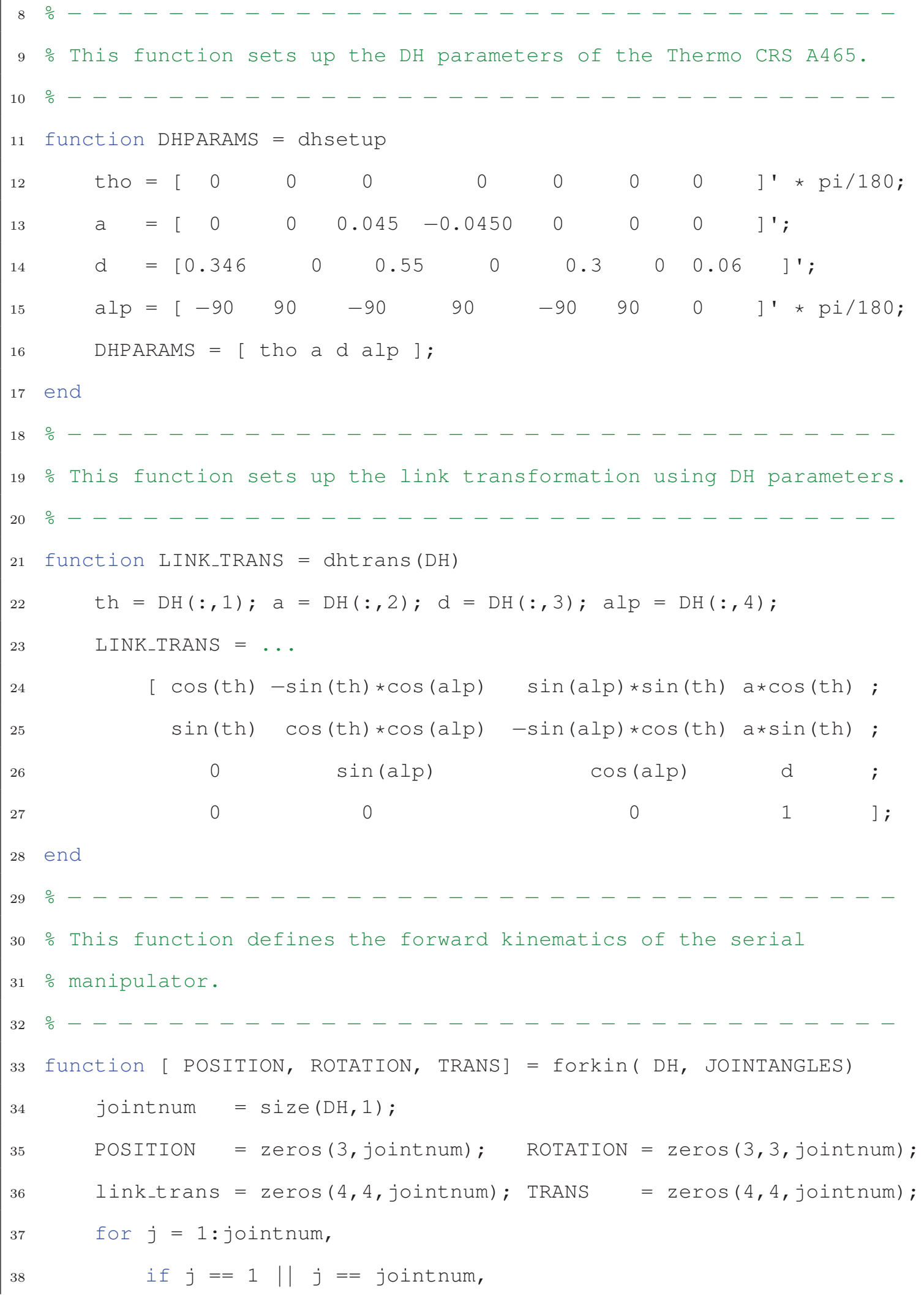




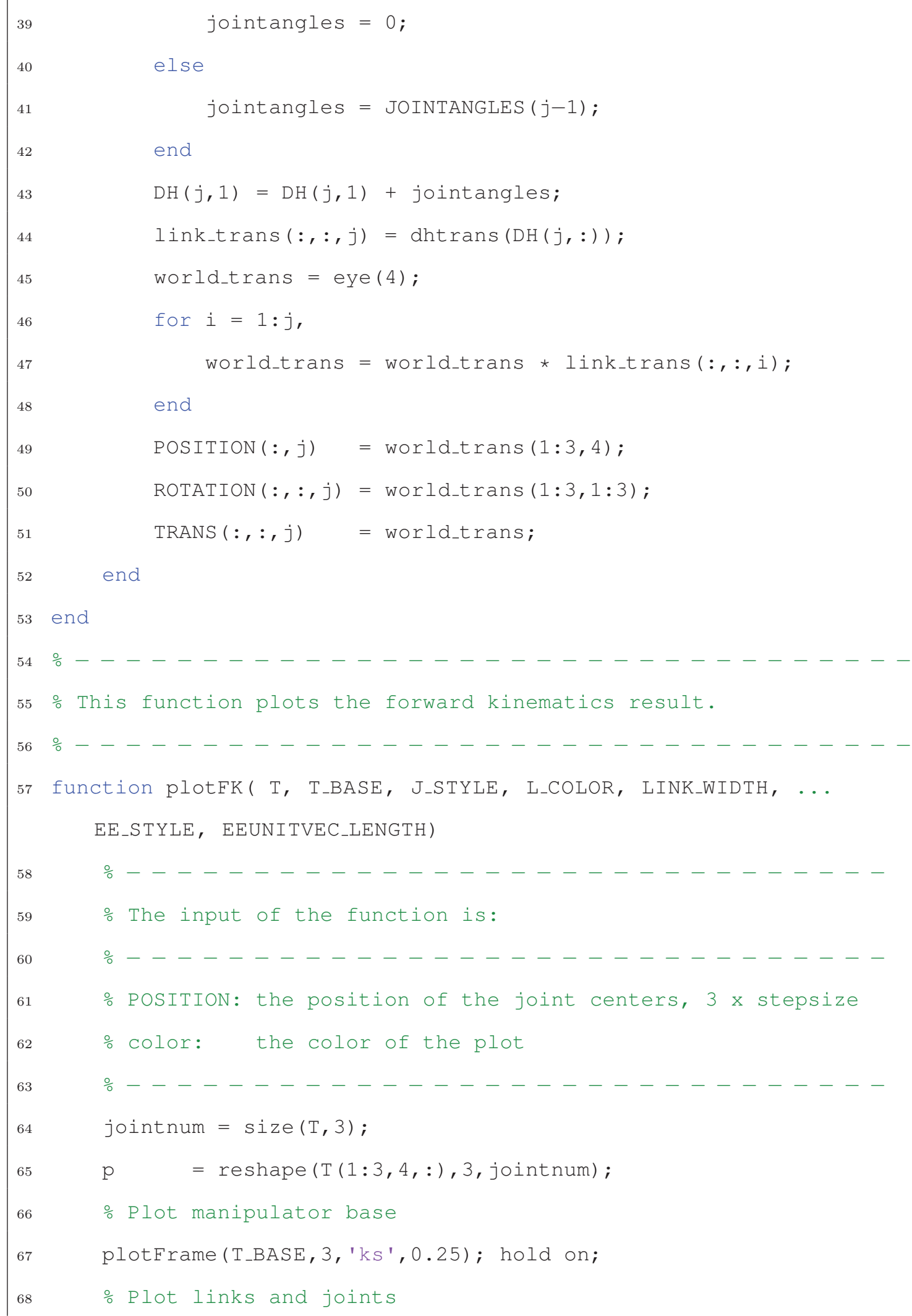




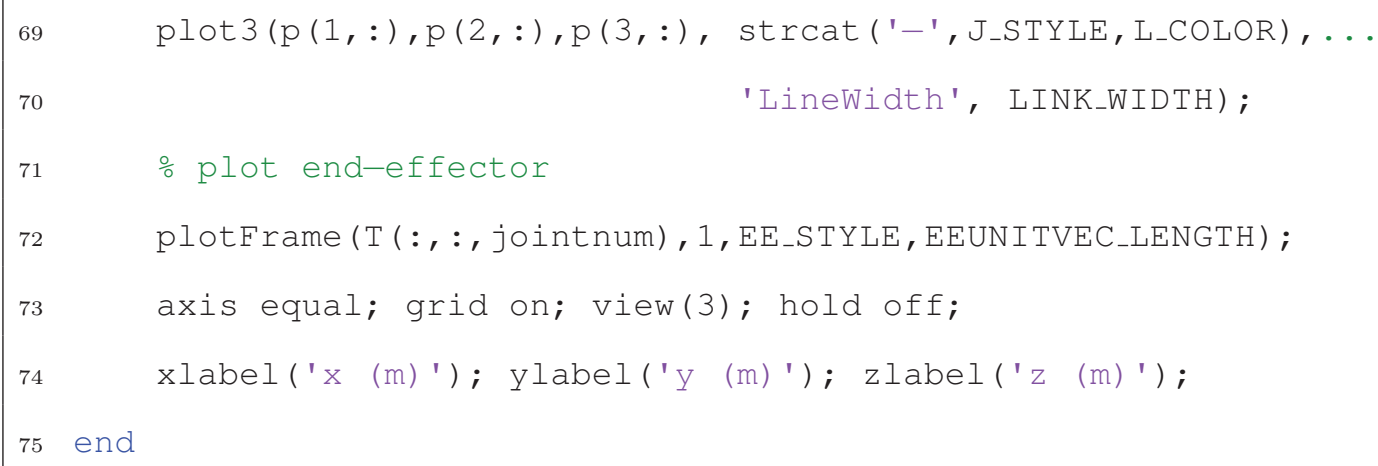




\section{Appendix D}

\section{CRS Functions in Python Code}

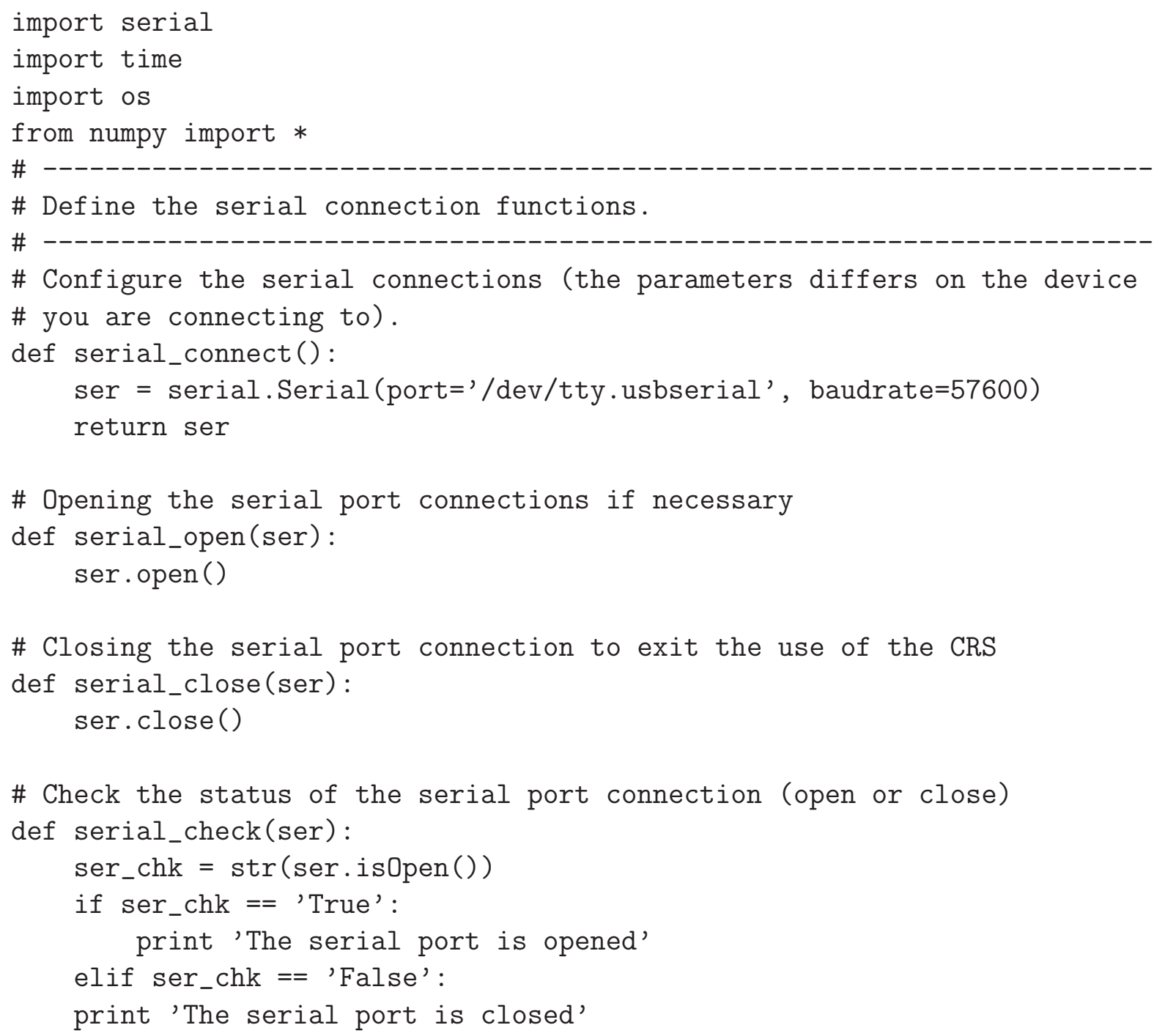




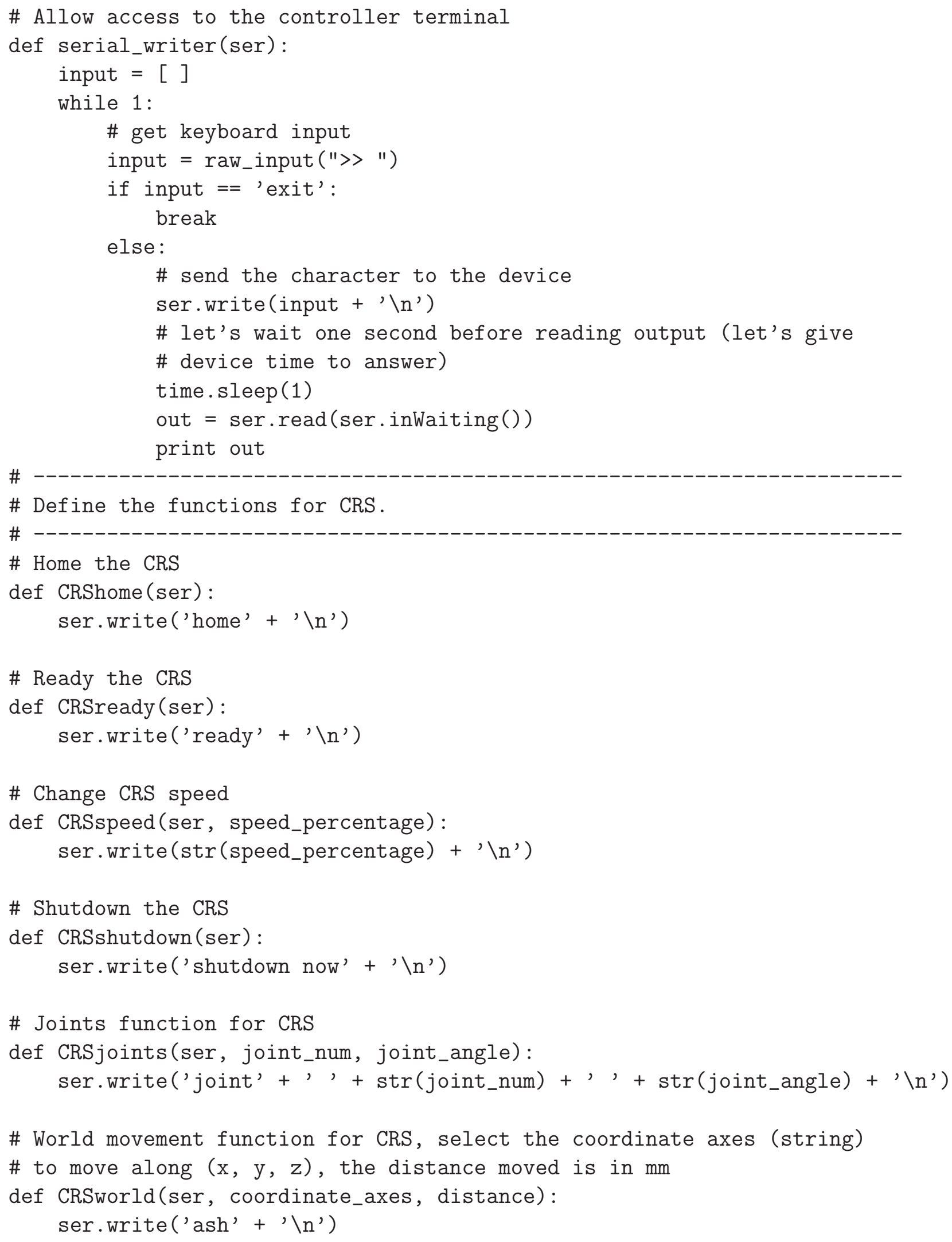




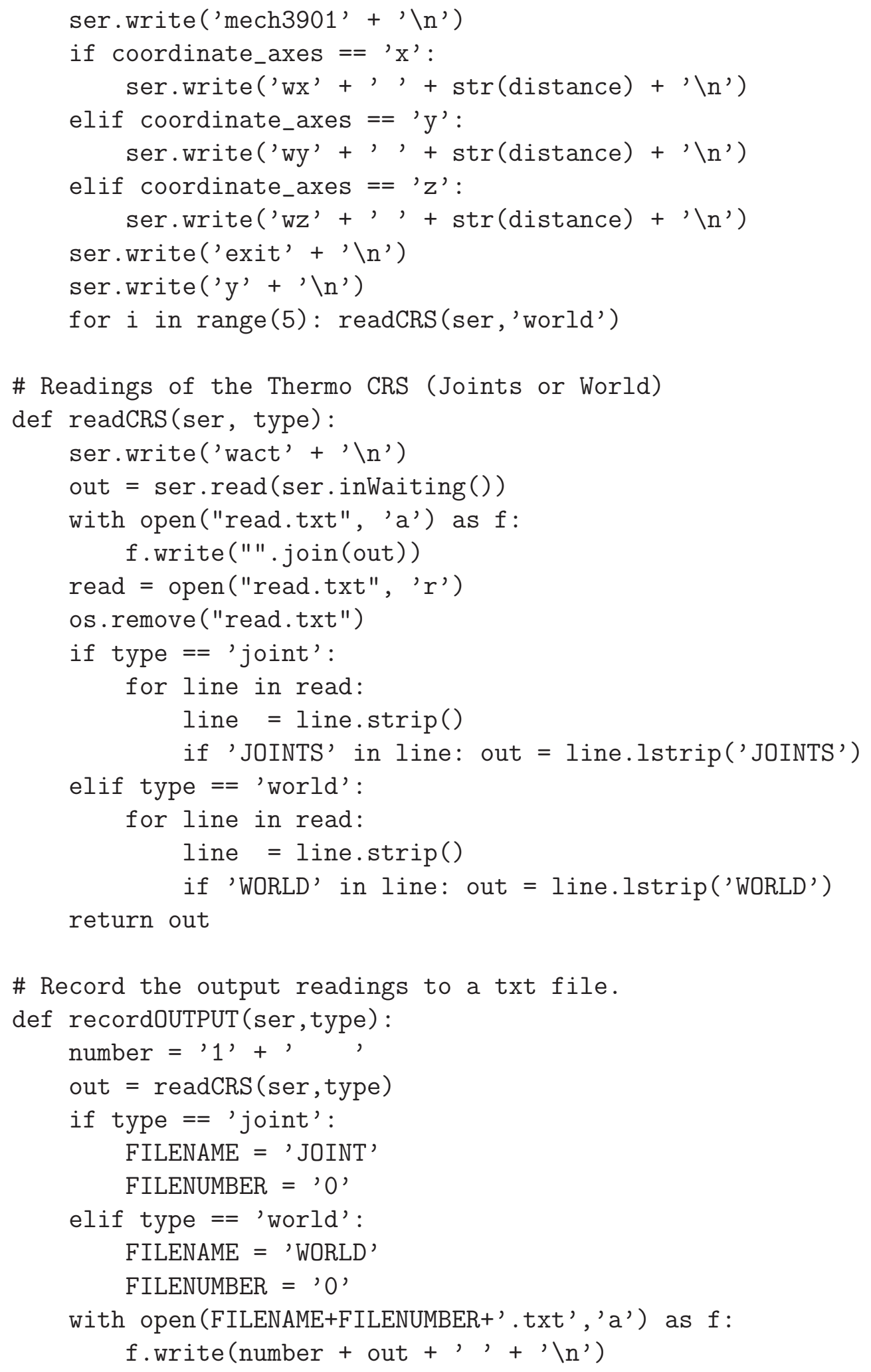




\section{Appendix E}

\section{OpenCV Written in Python for Camera Calibration}

camera_calibration.py

import numpy as $n p$

import cv2

import $\mathrm{cV} 2 . \mathrm{cV}$ as $\mathrm{CV}$

from video import create_capture \# py-module from pycv example.

from common import clock, draw_str \# py-module from pycv example.

from math import *

def cam_cal(IMAGES, SQUARESIZE, PATTERNSIZE) :

\#

\# This function calibrates the camera of interest.

\#

\# IMAGES: The images file name inputted into the function.

\# SQUARESIZE: The physical square size of the board pattern.

\# PATTERNSIZE: The number of internal corners on board (row by column).

\# 


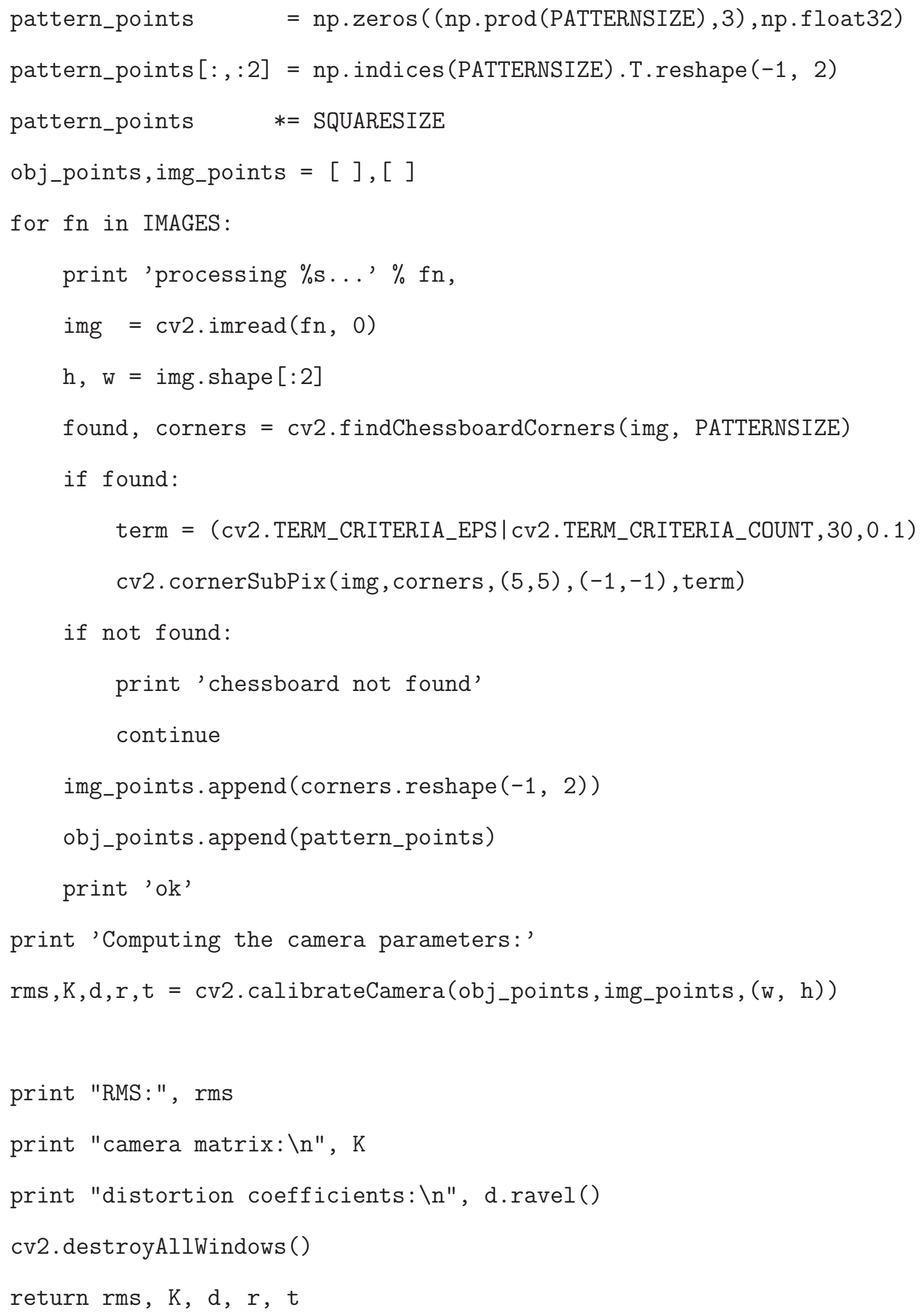


def camera_pose(IMAGES, SQUARESIZE, PATTERNSIZE, CAMERAMATRIX, DISTCOEFF):

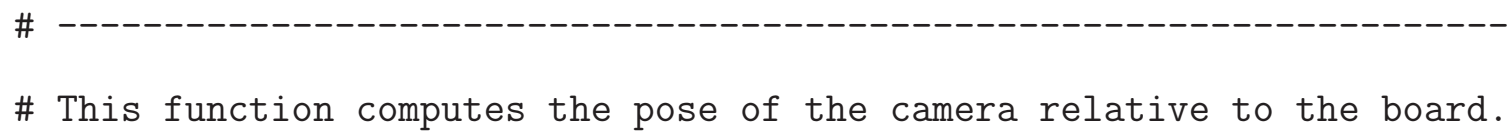




\section{CaptureBoard.py}

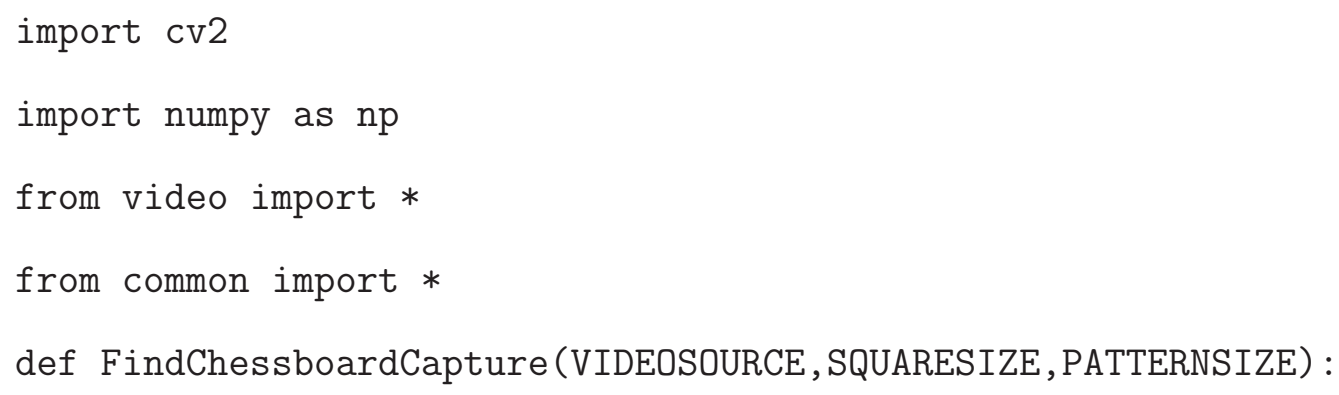




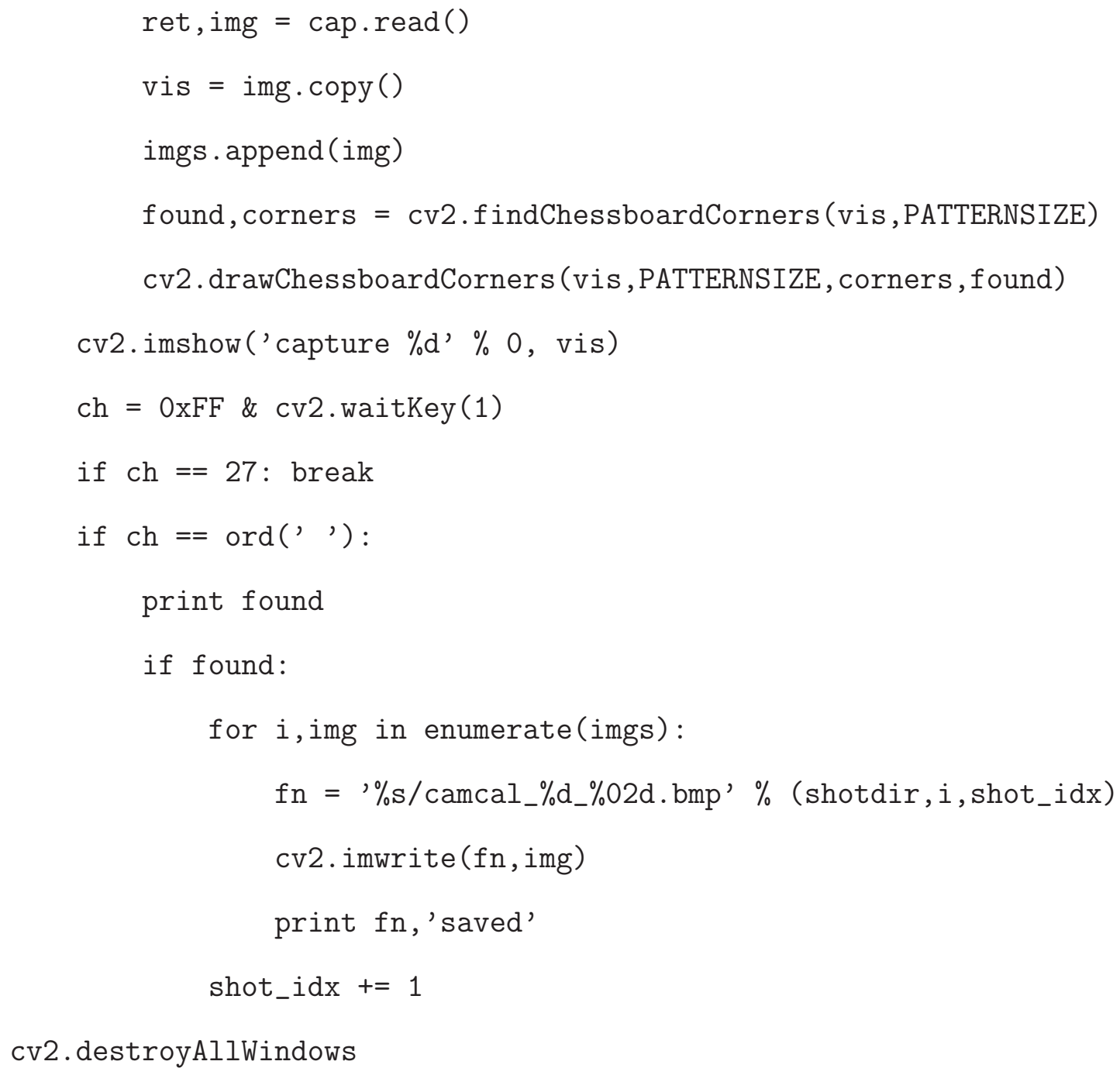


FindChessboardCapture (0,ipad_square, patternsize)

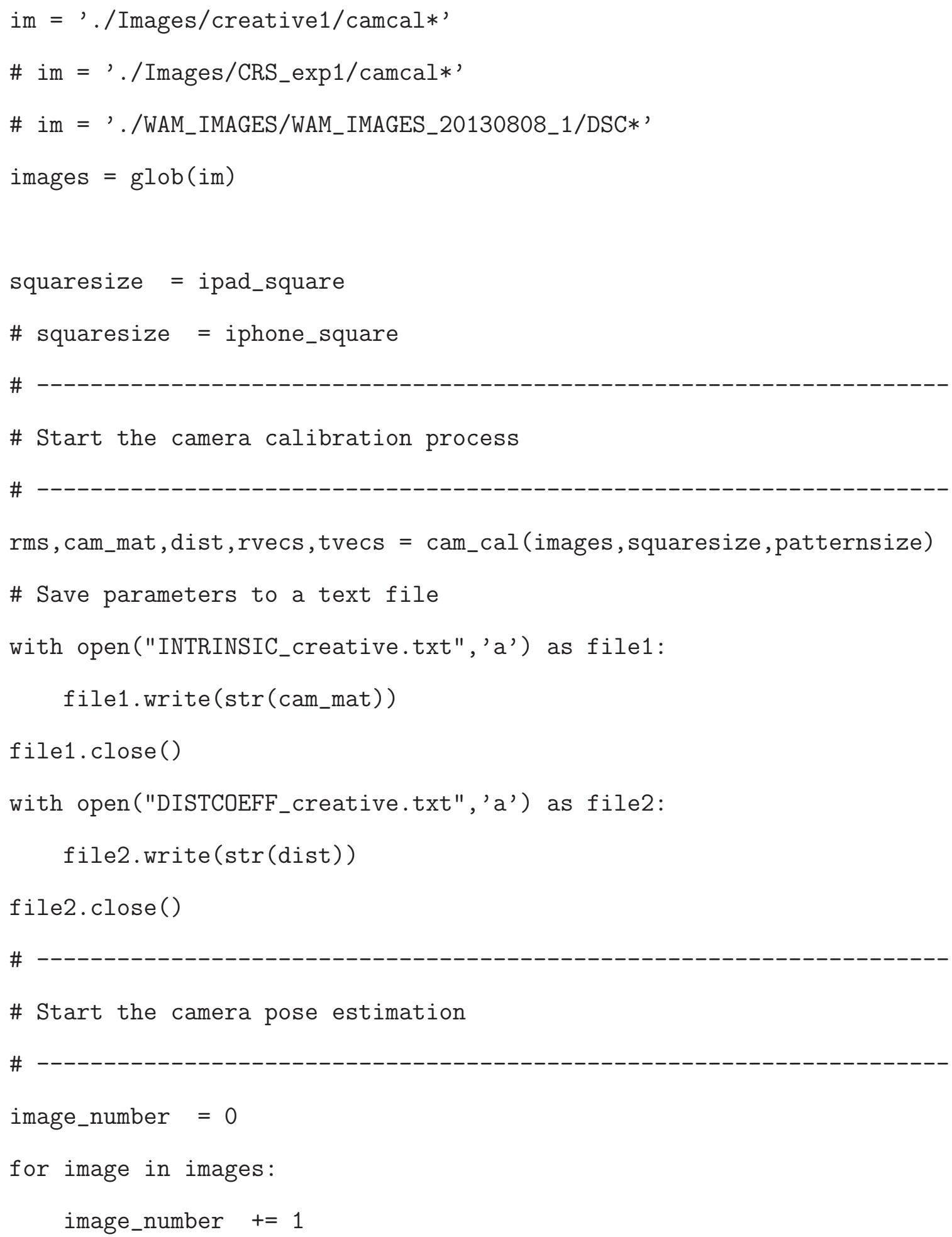




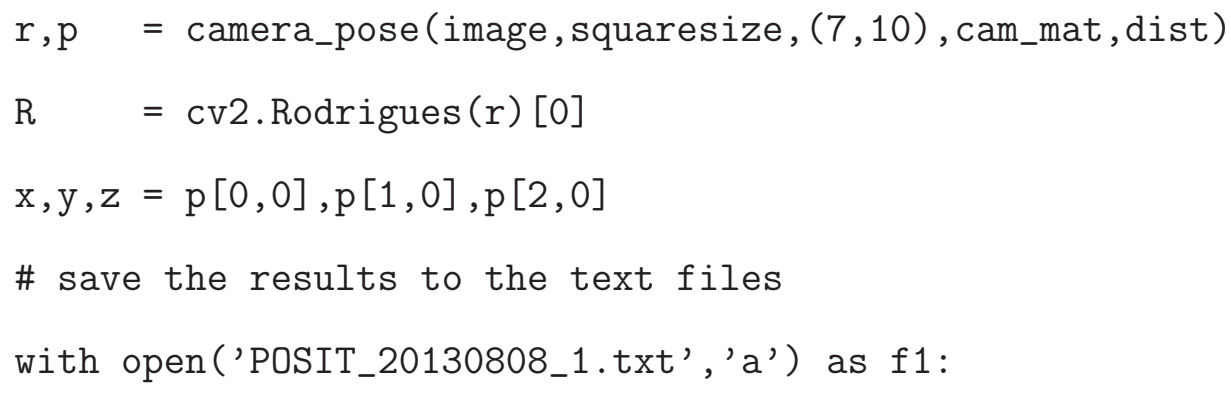

